\title{
UNITAL DILATIONS OF COMPLETELY POSITIVE SEMIGROUPS
}

\author{
by
}

\author{
David J. Gaebler
}

\author{
$\underline{\text { An Abstract }}$ \\ Of a thesis submitted in partial fulfillment of the \\ requirements for the Doctor of Philosophy \\ degree in Mathematics \\ in the Graduate College of \\ The University of Iowa
}

May 2013

Thesis Supervisor: Professor Paul S. Muhly 


\begin{abstract}
Semigroups of completely positive maps arise naturally both in noncommutative stochastic processes and in the dynamics of open quantum systems. Since its inception in the 1970's, the study of completely positive semigroups has included among its central topics the dilation of a completely positive semigroup to an endomorphism semigroup. In quantum dynamics, this amounts to embedding a given open system inside some closed system, while in noncommutative probability, it corresponds to the construction of a Markov process from its transition probabilities. In addition to the existence of dilations, one is interested in what properties of the original semigroup (unitality, various kinds of continuity) are preserved.

Several authors have proved the existence of dilations, but in general, the dilation achieved has been non-unital; that is, the unit of the original algebra is embedded as a proper projection in the dilation algebra. A unique approach due to Jean-Luc Sauvageot overcomes this problem, but leaves unclear the continuity of the dilation semigroup. The major purpose of this thesis, therefore, is to further develop Sauvageot's theory in order to prove the existence of continuous unital dilations. This existence is proved in Theorem 5.4.9, the central result of the thesis.

The dilation depends on a modification of free probability theory, and in particular on a combinatorial property akin to free independence. This property is implicit in some of Sauvageot's original calculations, but a secondary goal of this thesis is to present it as its own object of study, which we do in chapter 2 .
\end{abstract}


Abstract Approved:

Thesis Supervisor

Title and Department

Date 
UNITAL DILATIONS OF COMPLETELY POSITIVE SEMIGROUPS

by

David J. Gaebler

A thesis submitted in partial fulfillment of the requirements for the Doctor of Philosophy degree in Mathematics in the Graduate College of The University of Iowa

May 2013

Thesis Supervisor: Professor Paul S. Muhly 
Copyright by

DAVID J. GAEBLER

2013

All Rights Reserved 
Graduate College

The University of Iowa

Iowa City, Iowa

CERTIFICATE OF APPROVAL

PH.D. THESIS

This is to certify that the Ph.D. thesis of

David J. Gaebler

has been approved by the Examining Committee for the thesis requirement for the Doctor of Philosophy degree in Mathematics at the May 2013 graduation.

Thesis Committee:

Paul S. Muhly, Thesis Supervisor

Palle E. T. Jorgensen

Raul E. Curto

Ionut Chifan

Charles Frohman 
Sicut lilium inter spinas,

sic amica mea inter filias 


\section{ACKNOWLEDGEMENTS}

Whatever I have achieved in life has depended on the assistance of countless others. For my mathematical development, I would like to thank my parents for teaching me both how to do math and how to enjoy it, and for their continuing encouragement after I left the nest. Among my undergraduate mentors I especially remember Michael Moody, who convinced me to become a mathematician; Art Benjamin, who awakened me to the showmanship inherent in good mathematical exposition; and Francis Su, who showed me how to be wise as well as smart. More recently, my advisor, Paul Muhly, has modeled the many dimensions of mathematical maturity; has been relentlessly encouraging at every step of my research, during both the discoveries and the setbacks; and has taken a sincere interest in my personal as well as professional well-being. I truly cannot thank him enough. Other mathematicians have been kind to me as well. Most closely related to this thesis, Orr Shalit and

Daniel Markiewicz have been patient in bringing me up to speed with the basics of CP-semigroups. And fellow Muhly disciples Jonas, Andrew, Travis, Jenni, Erin, and Rachael formed the cadre of compatriots without which graduate school would be harder than it already is.

The support staff at the University of Iowa have rendered invaluable assistance, and I would like especially to thank Margaret Driscol, Cindy Farthing, and Lucy Silag for their courtesy, professionalism, and expertise. 
As much as I enjoy mathematics for its own sake, my toil under the sun is made meaningful by my family. Two are indeed better than one, and a cord of five (so far!) strands is not easily broken. My wife Leslie has gone out of her way to free up my time and to cheer me on, even while she has her hands full caring for $3-\epsilon$ small children. An excellent wife is the crown of her husband, and my crown is more splendid than any man could deserve. I would also like to thank my sons for their patience during my busy times, and my in-laws for helping cover all the bases.

Most of all, thanks are due to God the Father, in whom we live and move and have our being; the Son, in whom are hidden all the treasures of wisdom and knowledge; and the Spirit, who enables all skilled workmen from Bezalel to the present day. SDG. 


\begin{abstract}
Semigroups of completely positive maps arise naturally both in noncommutative stochastic processes and in the dynamics of open quantum systems. Since its inception in the 1970's, the study of completely positive semigroups has included among its central topics the dilation of a completely positive semigroup to an endomorphism semigroup. In quantum dynamics, this amounts to embedding a given open system inside some closed system, while in noncommutative probability, it corresponds to the construction of a Markov process from its transition probabilities. In addition to the existence of dilations, one is interested in what properties of the original semigroup (unitality, various kinds of continuity) are preserved.

Several authors have proved the existence of dilations, but in general, the dilation achieved has been non-unital; that is, the unit of the original algebra is embedded as a proper projection in the dilation algebra. A unique approach due to Jean-Luc Sauvageot overcomes this problem, but leaves unclear the continuity of the dilation semigroup. The major purpose of this thesis, therefore, is to further develop Sauvageot's theory in order to prove the existence of continuous unital dilations. This existence is proved in Theorem 5.4.9, the central result of the thesis.

The dilation depends on a modification of free probability theory, and in particular on a combinatorial property akin to free independence. This property is implicit in some of Sauvageot's original calculations, but a secondary goal of this thesis is to present it as its own object of study, which we do in chapter 2 .
\end{abstract}




\section{TABLE OF CONTENTS}

\section{CHAPTER}

1 INTRODUCTION TO COMPLETELY POSITIVE SEMIGROUPS . 4

1.1 Completely Positive Maps, Completely Positive Semigroups, and Endomorphism Semigroups . . . . . . . . . . . . . . . . 4

1.2 Dilation . . . . . . . . . . . . . . . . 7

1.3 Motivation and Examples ................. . . . . . . . 8

1.4 Continuity Properties of Semigroups . . . . . . . . . . . . 14

1.4.1 $\mathrm{C}_{0}$-Semigroups . . . . . . . . . . . . . . . . . . 14

1.4.2 Completely Positive Semigroups . . . . . . . . . . . 21

1.5 Survey of Extant Results . . . . . . . . . . . . . . . 22

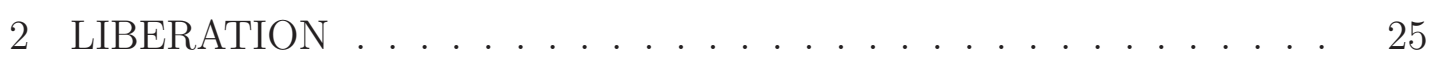

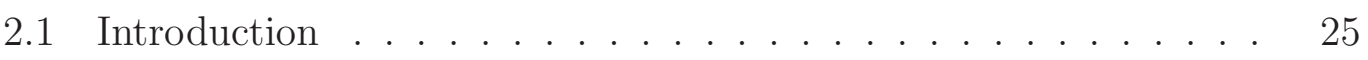

2.2 Background: Free Independence and Joint Moments . . . . . . . 26

2.3 Defining Liberation . . . . . . . . . . . . . . . . . . . . . . . 29

2.4 Right Liberation and Joint Moments . . . . . . . . . . . . . . . 32

2.5 Left Liberation and Joint Moments . . . . . . . . . . . . . . . 39

3 THE SAUVAGEOT PRODUCT ....................... 43

3.1 Introduction . . . . . . . . . . . . . . . 43

3.2 Sauvageot Products of Hilbert Spaces and Bounded Operators . 45

3.3 Sauvageot Products of $\mathrm{C}^{*}$-Algebras and $\mathrm{W}^{*}$-Algebras . . . . . . . 54

3.4 Induced Morphisms and Uniqueness . . . . . . . . . . . . . 60

3.5 Trivial Cases of the Sauvageot Product . . . . . . . . . . . 72

4 ALGEBRAIC C*-DILATIONS THROUGH ITERATED PRODUCTS 75

4.1 Introduction . . . . . . . . . . . . . . . . 75

4.2 Construction of the Inductive System and Limit . . . . . . . . . 78

4.2.1 Objects and Immediate-Tail Morphisms . . . . . . . . . . 78

4.2.2 General Morphisms . . . . . . . . . . . . . . . . 79

4.3 Endomorphisms of the Limit Algebra . . . . . . . . . . . 80

4.4 The Limit Retraction . . . . . . . . . . . . . . . . . . . 83

5 CONTINUOUS $W^{*}$-DILATIONS ................... 87

5.1 Introduction: The Problem of Continuity . . . . . . . . . 87 
5.1.1 Establishing the Problem: Discontinuity of the Existing Dilation . . . . . . . . . . . . . . 87

5.1.2 Fixing the Problem: Skorohod Space? . . . . . . . . . . 89

5.1.3 Fixing the Problem: Dilations on $L^{\infty} \ldots \ldots \ldots$. . . . 91

5.2 Moment Polynomials . . . . . . . . . . . . . . . . . 93

5.3 Continuity Properties of Moment Polynomials . . . . . . . . . 98

5.4 The Continuous Theorem . . . . . . . . . . . . . . . . . . 100

6 COVARIANT FILTRATIONS FOR SAUVAGEOT DILATIONS . . . 117

6.1 Introduction . . . . . . . . . . . . . . . . . 117

6.2 Strong Right-Liberation . . . . . . . . . . . . . . . . 118

6.3 Lifting a Retraction to the Sauvageot Product . . . . . . . . . 121

6.4 The Inductive System: Retractions Onto Initial Segments . . . . 125

6.5 Covariant Filtrations on $\mathrm{W}^{*}$-Dilations? . . . . . . . . . . 129

7 PRODUCT SYSTEMS $\ldots \ldots \ldots \ldots$

7.1 Introduction . . . . . . . . . . . . . . . . . . . . 132

7.2 Hilbert $\mathrm{C}^{*}$-Modules and Correspondences . . . . . . . . . 134

7.3 Units for Product Systems Associated With Sauvageot Dilations 136

A TABLE OF VALUES OF COLLAPSE AND MOMENT FUNCTIONS 138

A.1 Right-Liberation Collapse Functions . . . . . . . . . . . . . . 140

A.2 Right-Liberation Moment Functions . . . . . . . . . . . . . 143

A.3 Left-Liberation Collapse Functions . . . . . . . . . . . . . 147

A.4 Left-Liberation Moment Functions . . . . . . . . . . . . . 148

A.5 Moment Polynomials . . . . . . . . . . . . . . 150

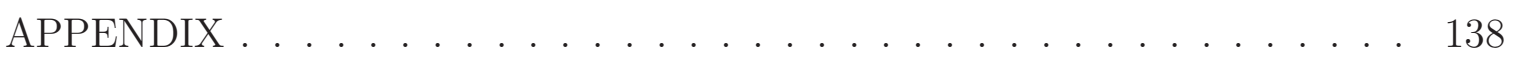

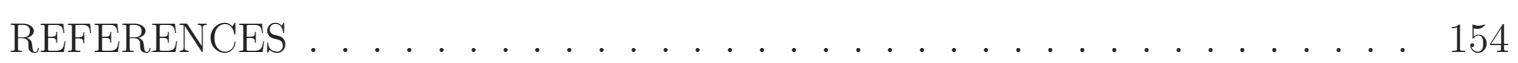




\section{PREFACE: BACKGROUND AND TERMINOLOGY}

This thesis is intended to be readable by a graduate student with a working knowledge of the fundamentals of functional analysis and operator algebras, but without prior exposure to the theory of completely positive maps or of operator semigroups. For instance, the preparation provide by Mac09] and [Zhu93] should be adequate, and that by [KR83] ample.

Following [Sak98, we distinguish between $\mathrm{W}^{*}$-algebras, which are abstractly defined as $\mathrm{C}^{*}$-algebras having a Banach-space predual (necessarily unique, as it turns out), and von Neumann algebras, which are concretely defined as weakly closed selfadjoint subalgebras of $B(H)$ for some Hilbert space $H$. In this convention, every von Neumann algebra is also a $\mathrm{W}^{*}$-algebra (with predual equal to a quotient of the predual $B(H)_{*} \simeq L^{1}(H)$ ), whereas every $\mathrm{W}^{*}$-algebra is isomorphic to some von Neumann algebra ([Sak98] 1.16.7). We depart somewhat from Sakai in referring to the weak-* topology on a $\mathrm{W}^{*}$-algebra as the ultraweak topology, which he calls the $\sigma$-topology or weak topology, and the topology induced by the seminorms $x \mapsto \phi\left(x^{*} x\right)$ for positive weak-* continuous functionals $\phi$ as the ultrastrong topology, which he calls the strong topology or s-topology. In the case of a von Neumann algebra, these topologies coincide with the ultraweak and ultrastrong operator topologies as usually defined (Sak98 1.15.6), and hence also with the weak and strong operator topologies on bounded subsets ([Sak98] 1.15.2). Because of this latter fact, we sometimes drop the "ultra" and refer merely to the weak and strong topologies when working on a bounded subset of a $\mathrm{W}^{*}$-algebra. We shall also make (rare) use of the ultrastrong-* 
topology, in which $x_{\nu} \rightarrow x$ iff $x_{\nu} \rightarrow x$ strongly and $x_{\nu}^{*} \rightarrow x^{*}$ strongly. Among the properties of these topologies that we will need are the following:

- Multiplication is separately continuous in both the ultraweak and ultrastrong topologies. However, it is jointly continuous in neither. On bounded sets, multiplication is jointly strongly and strong-* continuous, but not jointly weakly continuous.

- The adjoint map $x \mapsto x^{*}$ is ultraweakly continuous, but not ultrastrongly nor even strongly continuous.

- On bounded subsets, one may relate the weak and strong topologies as follows: $x_{\nu} \rightarrow x$ strongly iff $x_{\nu} \rightarrow x$ weakly and $x_{\nu}^{*} x_{\nu} \rightarrow x^{*} x$ weakly.

- The Kaplansky density theorem: If $A$ is a $\mathrm{W}^{*}$-algebra and $A_{0} \subset A$ an ultraweakly dense ${ }^{*}$-subalgebra, then the unit ball of $A_{0}$ is strong-* dense in the unit ball of $A$. In the case of a von Neumann algebra, the hypothesis of ultraweak density may be replaced by WOT-density.

A linear map between $\mathrm{W}^{*}$-algebras which is continuous with respect to their ultraweak topologies is called normal; if the map in question is positive, this is equivalent to the property of preserving upward-convergent nets (in this case weak and strong convergence are equivalent) of positive elements, that is, a positive linear map is normal iff $\phi\left(x_{\alpha}\right) \uparrow \phi(x)$ whenever $x_{\alpha} \uparrow x$ (Con00] Corollary 46.5). A C*-isomorphism between two $\mathrm{W}^{*}$-algebras is automatically normal, but a ${ }^{*}$-homomorphism or completely positive map need not be. 
We refer to a $\mathrm{W}^{*}$-algebra $\mathcal{A}$ as separable if its predual $\mathcal{A}_{*}$ is a separable Banach space; this can be shown to be equivalent to numerous other conditions, including the separability of either $\mathcal{A}$ or its unit ball in either the weak or strong topologies, and the existence of a faithful normal representation of $\mathcal{A}$ on a separable Hilbert space. A related but strictly weaker property is that of countable decomposability, which can be defined as the property that every mutually orthogonal family of nonzero projections in $\mathcal{A}$ is at most countable; this is equivalent to the existence of a faithful state, the existence of a faithful normal state, or the strong metrizability of the unit ball ([Bla06] III.2.2.27).

Additional background material, such as free probability and Hilbert $\mathrm{C}^{*}$ modules, will be addressed in the chapters where these topics first appear.

Throughout, we use the boldface symbol 1 to denote the unit of an algebra, while 1 will denote the natural number. 
CHAPTER 1

\section{INTRODUCTION TO COMPLETELY POSITIVE SEMIGROUPS}

\subsection{Completely Positive Maps, Completely Positive Semigroups, and Endomorphism Semigroups}

In this section we introduce the basic objects of study.

Definition 1.1.1. Let $A, B$ be $C^{*}$-algebras and $\phi: A \rightarrow B$ a linear map. We say that $\phi$ is

1. positive if it maps positive elements of $A$ to positive elements of $B$,

2. $n$-positive if the map $I_{n} \otimes \phi: M_{n}(\mathbb{C}) \otimes A \rightarrow M_{n}(\mathbb{C}) \otimes B$ is positive, and

3. completely positive if $\phi$ is $n$-positive for all $n \geq 1$.

We record here without proof some of the important properties of completely positive maps.

- If either $A$ or $B$ is commutative, the map $\phi: A \rightarrow B$ is positive iff it is completely positive. ([Pau02] Theorems 3.9 and 3.11)

- Every positive linear map is a $*^{*}$ map, that is, has the property that $\phi(a)^{*}=$ $\phi\left(a^{*}\right)$ for all $a \in A .($ Pau02] Exercise 2.1)

- If $\phi$ is 2-positive (so, in particular, if $\phi$ is completely positive), then $\phi(a)^{*} \phi(a) \leq$ $\phi\left(a^{*} a\right)$ for all $a \in A$. This is known as the Schwarz inequality for 2-positive maps. ([Pau02] Proposition 3.3) 
- If $A$ and $B$ are $\mathrm{W}^{*}$-algebras, a completely positive map $\phi: A \rightarrow B$ is normal iff it is strongly continuous. ([Bla06] Proposition III.2.2.2). Strong continuity is equivalent to ultrastrong because of the boundedness of the map.

- If $\phi: A \rightarrow B(H)$ is a completely positive map, there exists a triple $(K, V, \pi)$, unique up to isomorphism, such that

1. $K$ is a Hilbert space

2. $V: H \rightarrow K$ is a linear map such that $\|\phi\|=\|V\|^{2}$

3. $\pi: A \rightarrow B(K)$ is a *-homomorphism such that $V^{*} \pi(a) V=\phi(a)$ for all $a \in A$

and with the additional minimality property that $\overline{\pi(A) V H}=K$. The triple $(H, V, \pi)$ is called the minimal Stinespring dilation of $\phi$. If $\phi$ is unital, $V$ is an isometry; if $\phi$ is normal, so is $\pi$. This is known as Stinespring's dilation theorem ([Sti55], Pau02] Theorem 4.1, Bla06] Theorems II.6.9.7 and III.2.2.4).

Definition 1.1.2. Let $\mathcal{A}$ be a $\mathrm{C}^{*}$-algebra (resp. $\mathrm{W}^{*}$-algebra).

1. A cp-semigroup on $\mathcal{A}$ is a family $\left\{\phi_{t}: t \in[0, \infty)\right\}$ of (normal) completely positive contractive linear maps $\phi_{t}: \mathcal{A} \rightarrow \mathcal{A}$ such that $\phi_{0}=\mathrm{id}_{\mathcal{A}}$ and

$$
\phi_{t} \circ \phi_{s}=\phi_{t+s}
$$

for all $s, t \geq 0$. 
2. An e-semigroup on $\mathcal{A}$ is a cp-semigroup in which each $\phi_{t}$ is a ${ }^{*}$-endomorphism.

3. Capital letters (CP-semigroup, E-semigroup) indicate that for each $a \in \mathcal{A}$, $t \mapsto \phi_{t}(a)$ is a continuous function from $[0, \infty)$ to $\mathcal{A}$, where $\mathcal{A}$ is given the norm (resp. ultraweak) topology. We refer to this continuity property of the semigroup as strong continuity or point-norm continuity in the $\mathrm{C}^{*}$ case, and point-weak continuity in the $\mathrm{W}^{*}$ case.

4. A subscript of 0 ( $\mathbf{c p}_{0^{-}}$-semigroup, $\left.\mathbf{C P}_{0^{-}}, \mathbf{e}_{0^{-}}, \mathbf{E}_{0^{-}}\right)$indicates that $\mathcal{A}$ contains a unit $\mathbf{1}$ and that $\phi_{t}(\mathbf{1})=\mathbf{1}$ for all $t \geq 0$.

Remark 1.1.3. The term quantum Markov process or quantum Markov semigroup is sometimes used in the literature to describe $\mathrm{cp}-, \mathrm{cp}_{0^{-}}, \mathrm{CP}$, or $\mathrm{CP}_{0^{-}}$-semigroups; however, the usage is nonuniform as to which of these is indicated, so we adhere to the more precise notation above.

Remark 1.1.4. In the case where $\mathcal{A}$ is a $\mathrm{W}^{*}$-algebra, the definition of cp-semigroups and CP-semigroups remain unchanged when stated in terms of the strong topology rather than the weak topology. That is, each map $\phi_{t}$ is normal iff it is strongly continuous, as noted above; and, as we shall show in more detail below, the map $t \mapsto \phi_{t}(a)$ for fixed $a$ is continuous with respect to the weak topology on bounded subsets of $\mathcal{A}$ iff it is continuous with respect to the strong topology (that is, point-weak continuity is equivalent to point-strong continuity).

Definition 1.1.5. Let $\phi=\left\{\phi_{t}\right\}$ be a cp-semigroup on $A$. An invariant state for $\phi$ 
is a state $\omega: A \rightarrow \mathbb{C}$ with the property

$$
\forall t \geq 0: \quad \omega \circ \phi_{t}=\omega
$$

\subsection{Dilation}

In this section we introduce the ways in which cp-semigroups and e-semigroups may be related to each other.

Definition 1.2.1. Let $A, B$ be $\mathrm{C}^{*}$-algebras.

1. A conditional expectation on $A$ is a linear map $E: A \rightarrow A$ such that $E^{2}=E$, $E\left(x^{*}\right)=E(x)^{*}$ for all $x \in A$, and $\|E\|=1$.

2. An embedding from $A$ to $B$ is an injective *-homomorphism from $A$ to $B$.

3. Given an embedding $i: A \rightarrow B$, a retraction with respect to $i$ is a completely positive map $e: B \rightarrow A$ such that $e \circ i=\mathrm{id}_{A}$.

Remark 1.2.2. A linear map $E: A \rightarrow A$ is a conditional expectation iff it is a completely positive contraction and is a bimodule map over its range, i.e. has the property that $E(E(a) x)=E(a) E(x)=E(a E(x))$ for all $a, x \in A$; this is known as Tomiyama's theorem ([Tom57]). As a result, if $i: A \rightarrow B$ is an embedding and $e: B \rightarrow A$ a corresponding retraction, then $i \circ e$ is a conditional expectation on $B$ with range $i(A)$. Hence, the distinction between a retraction and a conditional expectation is precisely the distinction between identifying $A$ as a subalgebra of $B$, and explicitly writing an inclusion map from $A$ to $B$. The difference is a matter of taste; we generally follow the latter approach. 
Definition 1.2.3. Let $\phi=\left\{\phi_{t}\right\}$ be a cp-semigroup on a $\mathrm{C}^{*}$-algebra $\mathcal{A}$. An e-dilation of $(A, \phi)$ is a tuple $(\mathfrak{A}, i, \mathbb{E}, \sigma)$ where $\mathfrak{A}$ is a $\mathrm{C}^{*}$-algebra, $i: A \rightarrow \mathfrak{A}$ an embedding, $\mathbb{E}: \mathfrak{A} \rightarrow A$ a retraction with respect to $i$, and $\sigma=\left\{\sigma_{t}\right\}$ an e-semigroup on $\mathfrak{A}$, satisfying

$$
\forall t \geq 0: \quad \phi_{t}=\mathbb{E} \circ \sigma_{t} \circ i
$$

We summarize the relationship in the following diagram:

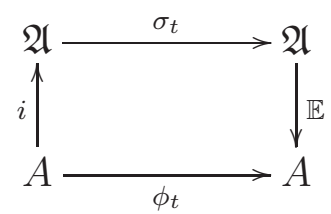

We call $(\mathfrak{A}, i, \mathbb{E}, \sigma)$ a strong e-dilation if it satisfies $\mathbb{E} \circ \sigma_{t}=\phi_{t} \circ \mathbb{E}$, corresponding to the diagram

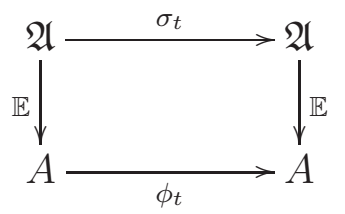

Note that this implies

$$
\phi_{t}=\phi_{t} \circ \mathbb{E} \circ i=\mathbb{E} \circ \sigma_{t} \circ i
$$

so that every strong dilation is a dilation, but the converse does not always hold. An $\mathrm{e}_{0}$-dilation of a $\mathrm{cp}_{0}$-semigroup is said to be unital if $i(\mathbf{1})=\mathbf{1}$.

\subsection{Motivation and Examples}

Example 1.3.1. Let $H$ be a Hilbert space and $\left\{T_{t}\right\}$ a semigroup of contractions on $H$. Then the maps $\phi_{t}: B(H) \rightarrow B(H)$ defined by

$$
\phi_{t}(X)=T_{t}^{*} X T_{T}
$$


form a cp-semigroup. It is a $\mathrm{cp}_{0}$-semigroup iff all the $T_{t}$ are isometries, an e-semigroup iff all the $T_{t}$ are coisometries, and hence an $\mathrm{e}_{0}$-semigroup iff all the $T_{t}$ are unitaries. If $T_{t}$ is strongly continuous, in that $t \mapsto T_{t}$ is continuous with respect to the strong operator topology on $B(H)$, then $\left\{\phi_{t}\right\}$ is a CP-semigroup.

A theorem of Cooper ([Coo47] $)$ states that, given a strongly continuous contraction semigroup $\left\{T_{t}\right\}$ on $H$, there exist a Hilbert space $K$, an isometry $V: H \rightarrow K$, and a strongly continuous group $\left\{U_{t}\right\}$ of unitaries on $K$ such that

$$
T_{t}=V^{*} U_{t} V .
$$

If the $T_{t}$ are isometries, one obtains the stronger condition

$$
V T_{t}=U_{t} V .
$$

Given the Cooper dilation of the semigroup $\left\{T_{t}\right\}$, one can then define

1. the $\mathrm{E}_{0}$-semigroup $\left\{\alpha_{t}\right\}$ on $B(K)$ by $\alpha_{t}(Y)=U_{t}^{*} Y U_{t}$

2. the non-unital embedding $i: B(H) \rightarrow B(K)$ by $i(X)=V X V^{*}$

3. the retraction $\mathbb{E}: B(K) \rightarrow B(H)$ by $\mathbb{E}(Y)=V^{*} Y V$

Then $\left(B(K), i, \mathbb{E},\left\{\alpha_{t}\right\}\right)$ is an $\mathrm{E}_{0}$-dilation of $\left(B(H),\left\{\phi_{t}\right\}\right)$.

This example plays a role in the general theory; for instance, Evans and Lewis prove their dilation theorem ([EL77]) by showing that certain more general semigroups are equivalent to those of the form $X \mapsto T_{t}^{*} X T_{t}$, and then applying Cooper dilation.

Example 1.3.2. In (one of the axiomatizations of) quantum mechanics, every physical system corresponds to a von Neumann algebra $\mathcal{A}$, with states of the system 
corresponding to positive elements of $\mathcal{A}$ of trace 1 . A physical transformation of the system must map states to states and hence, in particular, must be a positive map; a continuous-time evolution of the system corresponds therefore to a semigroup of positive maps. If the system is entangled with an environment, a physical transformation of the composite system must map composite states to composite states, which implies complete positivity of the restriction to the original system; hence, a continuous-time evolution of such an open quantum system is represented by a semigroup of completely positive maps. Continuity requirements are also natural to impose in this setting as one of the physical axioms.

Actually, the representation of such a system as a completely positive semigroup represents an approximation to a more general master equation, which approximation holds under various simplifying physical assumptions such as those of "weak coupling" or a "singular reservoir." Completely positive semigroups arise, for instance, in quantum thermodynamics, where the environment may be regarded as an infinite "heat bath" whose self-interactions are much faster than those of the system under study. For more on these matters see [Haa73], [Dav74], [GKS76], [Lin76], [Dav76], EL77], and [AJP06]. In the thermodynamic context one typically assumes the existence of a normal $\phi$-invariant state $\omega$ on $\mathcal{A}$, representing a thermodynamic equilibrium of the system; correspondingly, one is interested in dilations $(\mathfrak{A}, i, \mathbb{E}, \sigma)$ for which there exists a normal $\sigma$-invariant state $\varpi$ on $\mathfrak{A}$, which dilates $\omega$ in the sense that $\varpi \circ i=\omega$. In the case of a strong dilation this is automatic, as one can simply 
define $\varpi=\omega \circ \mathbb{E}$, and it follows that

$$
\varpi \circ \sigma_{t}=\omega \circ \mathbb{E} \circ \sigma_{t}=\omega \circ \phi_{t} \circ \mathbb{E}=\omega \circ \mathbb{E}=\varpi
$$

In this setting, dilation is a way of relating the dynamics of an open (or "dissipative") system to the dynamics of a closed (or "non-dissipative") system containing it.

Example 1.3.3. Let $\mathcal{A}$ be a commutative unital $\mathrm{C}^{*}$-algebra, and let $S$ be the maximal ideal space of $\mathcal{A}$, so that $\mathcal{A} \simeq C(S)$. Let $\left\{P_{t}\right\}$ be a $\mathrm{CP}_{0}$-semigroup on $\mathcal{A}$. By Riesz representation we obtain for each $t \geq 0$ and each $x \in S$ a measure $p_{t, x}$ characterized by the property

$$
\forall f \in C(S): \quad \int_{S} f(y) d p_{t, x}(y)=\left(P_{t} f\right)(x) .
$$

Moreover, since $P_{t} f$ is a continuous function, the family $\left\{p_{t, x}\right\}$ varies weak-* continuously in $x$. The property $P_{0}=$ id implies that $p_{0, x}$ is the point mass at $x$, and the semigroup property $P_{s+t}=P_{s} P_{t}$ implies the property

$$
p_{t+s, x}(E)=\int_{S} p_{s, y}(E) d p_{t, x}(y),
$$

sometimes referred to as the Chapman-Kolmogorov equation.

Let $\mathscr{S}$ denote the path space $S^{[0, \infty)}$, and $\mathfrak{A}=C(\mathscr{S})$. We have the embedding $i: \mathcal{A} \rightarrow \mathfrak{A}$ given by $i(f)(\mathfrak{p})=f(\mathfrak{p}(0))$. By the Stone-Weierstrass theorem, the *subalgebra $\mathfrak{A}_{0} \subset \mathfrak{A}$ consisting of finite sums of functions of the form $f_{1}^{\left(t_{1}\right)} \cdots f_{n}^{\left(t_{n}\right)}$, where for a path $\mathfrak{p} \in \mathscr{S}$ the value of $f_{i}^{\left(t_{i}\right)}$ depends only on $\mathfrak{p}\left(t_{i}\right)$, is dense in $\mathfrak{A}$. We define a unital linear map $\mathbb{E}_{0}: \mathfrak{A}_{0} \rightarrow \mathcal{A}$ on $\mathcal{A}_{0}$ by

$$
\mathbb{E}_{0}\left[f_{1}^{\left(t_{1}\right)} \cdots f_{n}^{\left(t_{n}\right)}\right]=f_{n} P_{t_{n}-t_{n-1}}\left(f_{n-1} P_{t_{n-1}-t_{n-2}}\left(\cdots P_{t_{2}-t_{1}}\left(f_{1}\right)\right) \cdots\right)
$$


Clearly $E_{0} \circ i=\operatorname{id}_{\mathcal{A}}$. We will show shortly that $\mathbb{E}_{0}$ is well-defined and contractive, so that it extends to a unital contractive (hence positive, hence completely positive) linear map $\mathbb{E}: \mathfrak{A} \rightarrow \mathcal{A}$ which satisfies $\mathbb{E} \circ i=\mathrm{id}_{\mathcal{A}}$ and is therefore a retraction with respect to $i$.

We define for each $t \geq 0$ the continuous maps $\lambda_{t}: \mathscr{S} \rightarrow \mathscr{S}$ by $\left(\lambda_{t} \mathfrak{p}\right)(s)=$ $\mathfrak{p}(s+t)$, and the corresponding *-endomorphisms $\sigma_{t}: \mathfrak{A} \rightarrow \mathfrak{A}$ by $\sigma_{t} f=f \circ \lambda_{t}$. It is immediate from the above that $\mathbb{E} \circ \sigma_{t} \circ i=P_{t}$, so that we have obtained a unital e-dilation of our $\mathrm{CP}_{0}$-semigroup.

Given any regular Borel probability measure $\mu_{0}$ on $S$, we obtain through Riesz representation a regular Borel probability measure $\mu$ on $\mathscr{S}$ characterized by the property

$$
\forall f \in \mathfrak{A}: \quad \int_{\mathscr{S}} f d \mu=\int_{S}(\mathbb{E} f) d \mu_{0} .
$$

This then implies that

$$
\forall f \in \mathcal{A}: \quad\left(P_{t} f\right)(x)=\mathcal{E}[f(\mathfrak{p}(t)) \mid \mathfrak{p}(0)=x]
$$

where $\mathcal{E}$ denotes conditional expectation in the probabilistic sense, so that we have constructed a Markov process $\{\mathfrak{p}(t)\}$ with specified transition probabilities. We thus obtain a $\mathrm{C}^{*}$-algebraic version of the classical Daniell-Kolmogorov construction, at least in the context of Feller processes rather than general Markov processes.

We now consider an alternate perspective on the same construction, which enables us easily to prove that $\mathbb{E}_{0}$ is well-defined and contractive, and simultaneously offers a preview of the techniques used in this thesis. For each nonempty finite subset 
$\gamma \subset[0, \infty)$ let $\mathcal{A}_{\gamma}$ denote a tensor product of $|\gamma|$ copies of $C(S)$. For $\beta \subset \gamma$ we obtain unital embeddings $\mathcal{A}_{\beta} \rightarrow \mathcal{A}_{\gamma}$ as follows: Writing $\gamma$ as a disjoint union $\beta \cup \gamma^{\prime}$, identify $\mathcal{A}_{\gamma}$ with $\mathcal{A}_{\beta} \otimes \mathcal{A}_{\gamma^{\prime}}$ and embed via $f \mapsto f \otimes 1$. This yields an inductive system and, using the general fact that $C(X \times Y) \simeq C(X) \otimes C(Y)$ for compact Hausdorff spaces $X$ and $Y$, we see that $\lim _{\rightarrow} \mathcal{A}_{\gamma}$ is isomorphic to $\mathfrak{A}$. The domain of $\mathbb{E}_{0}$ is the union of the images of all the $\mathcal{A}_{\gamma}$ inside $\mathfrak{A}$, and the well-definedness and contractivity of $\mathbb{E}_{0}$ reduce, by induction, to the well-definedness and contractivity of the maps $\theta_{t}: C(S) \otimes C(S)$ given on simple tensors by $\theta_{t}(f \otimes g)=\left(P_{t} f\right) g$. But such a map $\theta_{t}$ may be equivalently defined as

$$
\left(\theta_{t} F\right)(x)=\int_{S} F(y, x) d p_{t, x}(y)
$$

which obviously yields a well-defined contraction on $C(S) \otimes C(S)$.

We note that the e-semigroup $\left\{\sigma_{t}\right\}$ is not continuous, even when the original semigroup $\left\{P_{t}\right\}$ is; that is, we obtain only an $\mathrm{e}_{0}$-dilation, not an $\mathrm{E}_{0}$-dilation, of a $\mathrm{CP}_{0}$-semigroup. We shall return to this point in chapter 5 .

Remark 1.3.4. We view the last two examples as representing the two major streams of thought which motivate the study of the dilation theory of completely positive semigroups. On the one hand, in the physics setting such a dilation corresponds to an embedding of an open quantum system inside some closed quantum system. On the other hand, we have seen that dilating a $\mathrm{CP}_{0}$-semigroup defined an a commutative $\mathrm{C}^{*}$-algebra amounts to construction of a Markov process; hence, we may think of dilations of general $\mathrm{CP}_{0}$-semigroups as a way of constructing "noncommutative Markov processes." 


\subsection{Continuity Properties of Semigroups}

In this section we examine in greater detail the continuity properties of completely positive semigroups, beginning with more general considerations regarding contraction semigroups on Banach spaces.

\subsection{1 $\mathrm{C}_{0}$-Semigroups}

We recount here some of the essentials of the theory of contraction semigroups on Banach spaces, which can be found in [HP57, [DS88], BR87], and [EN06].

A semigroup $\{T(t)\}_{t \geq 0}$ of contractions on a Banach space $\mathfrak{X}$ is called a contraction semigroup. Such a semigroup is said to be

1. uniformly continuous if $t \mapsto T(t)$ is continuous with respect to the norm topology on $B(\mathfrak{X})$; that is, if $\lim _{t \rightarrow t_{0}}\left\|T(t)-T\left(t_{0}\right)\right\|_{B(\mathfrak{X})}=0$

2. strongly continuous if, for each $x \in \mathfrak{X}, t \mapsto T(t) x$ is continuous with respect to the norm topology on $\mathfrak{X}$; that is, if $\lim _{t \rightarrow t_{0}}\left\|T(t) x-T\left(t_{0}\right) x\right\|_{\mathfrak{X}}=0$ for each $x \in \mathfrak{X}$

3. weakly continuous if, for each $x \in \mathfrak{X}, t \mapsto T(t) x$ is continuous with respect to the weak topology on $\mathfrak{X}$; that is, if $\lim _{t \rightarrow t_{0}} \ell\left(T(t) x-T\left(t_{0}\right) x\right)=0$ for each $x \in \mathfrak{X}$ and each $\ell \in \mathfrak{X}^{*}$

In case $\mathfrak{X}$ is the dual of some other Banach space $\mathfrak{X}_{*}$, we define the semigroup to be

4. weak-* continuous if, for each $x \in \mathfrak{X}, t \mapsto T(t) x$ is continuous with respect to the weak-* topology on $\mathfrak{X}$; that is, if $\lim _{t \rightarrow t_{0}} \ell\left(T(t) x-T\left(t_{0}\right) x\right)=0$ for each $x \in \mathfrak{X}$ and each $\ell \in \mathfrak{X}_{*}$ 
These modes of continuity can, of course, be defined for other families $\{T(t)\}$ of operators which are not necessarily contractions and do not necessarily form a semigroup. In the case of contraction semigroups, however, it turns out that strong and weak continuity are equivalent ([EN06] Theorem 1.1.6). Furthermore, uniform continuity is too stringent a hypothesis to be attainable in most applications of interest, so that the bulk of the study of contraction semigroups revolves around strongly continuous contraction semigroups, also known as contractive $\mathbf{C}_{0}$-semigroups . More generally, one can study strongly continuous semigroups of bounded operators, but it can be easily shown that these may all be written as scalar-valued exponential functions times contraction semigroups, so that one reduces to the contractive case.

The most important object associated with a contractive $\mathrm{C}_{0}$-semigroup is its generator, the operator $\mathcal{L}$ on $\mathfrak{X}$ defined by the formula

$$
\mathcal{L} x=\lim _{t \rightarrow 0} t^{-1}[T(t) x-x]
$$

This is in general a closed densely defined unbounded operator, and in fact is bounded iff the semigroup is uniformly continuous. Furthermore, the generator satisfies the resolvent growth condition $\left\|(\lambda \mathbf{1}-\mathcal{L})^{-1}\right\| \leq \lambda^{-1}$ for all $\lambda>0$. The Hille-Yosida theorem provides a converse, stating that every closed densely defined operator satisfying this resolvent growth condition is the generator of some $\mathrm{C}_{0}$-semigroup. Intuitively, this semigroup is given by $T(t)=e^{t \mathcal{L}}$, but this exponential functional cannot be defined through the usual power series when $\mathcal{L}$ is unbounded; one can, 
however, write

$$
T(t) x=\lim _{n \rightarrow \infty}\left(\mathbf{1}-\frac{t}{n} \mathcal{L}\right)^{-n} x
$$

which is known as the Post-Widder inversion formula for $\mathrm{C}_{0}$-semigroups. We thus have a bijection between contractive $\mathrm{C}_{0}$-semigroups and closed densely defined operators satisfying a resolvent growth condition, with explicit formulas for both directions of the bijection.

A notable consequence of the semigroup property is the equivalence between certain notions of continuity and measurability. We define a family $\{T(t)\}$ of operators on $\mathfrak{X}$, equivalently viewed as a function $T:[0, \infty) \rightarrow B(\mathfrak{X})$, to be

1. uniformly measurable if $T$ is the a.e. norm limit of a sequence of countablyvalued functions from $[0, \infty)$ to $B(\mathfrak{X})$

2. strongly measurable if, for each $x \in \mathfrak{X}, t \mapsto T(t) x$ is the a.e. norm limit of a sequence of countably-valued functions from $[0, \infty)$ to $\mathfrak{X}$

3. weakly measurable if, for each $x \in \mathfrak{X}$ and $\ell \in \mathfrak{X}^{*}, t \mapsto \ell(T(t) x)$ is a measurable function from $[0, \infty)$ to $\mathbb{C}$

In case $\mathfrak{X}$ is the dual of another Banach space $\mathfrak{X}_{*}$, we also define $\{T(t)\}$ to be

4. weak-* measurable if, for each $x \in \mathfrak{X}$ and $\ell \in \mathfrak{X}_{*}, t \mapsto \ell(T(t) x)$ is a measurable function from $[0, \infty)$ to $\mathbb{C}$.

One might ask why we do not instead define the different types of measurability using the Borel $\sigma$-algebras generated by the corresponding continuity types; the short 
answer is that a better integration theory results from the definitions given here (the Bochner integral in the case of uniform measurability, the Pettis integral for the others).

It turns out that weak and strong measurability are equivalent when $\mathfrak{X}$ is separable ([HP57] Corollary 2, p. 73) and, when $\{T(t)\}$ is a contraction semigroup, both are equivalent to strong and weak continuity at times $t>0$ ([HP57] Theorem 10.2.3). This latter result is analogous to the fact that measurable solutions to the Cauchy functional equation $f(x+y)=f(x) f(y)$ on $\mathbb{R}$ are exponentials, and hence are continuous. However, strong measurability at $t=0$ is not enough to infer strong continuity at $t=0$, but requires the additional hypothesis that $\bigcup_{t>0} T(t) \mathfrak{X}$ be dense in $\mathfrak{X}$ ([HP57] Theorem 10.5.5).

A contraction semigroup $\{T(t)\}$ on $\mathfrak{X}$ induces an adjoint semigroup $\left\{T(t)^{*}\right\}$ on $\mathfrak{X}^{*}$ by the formula $\left(T(t)^{*} f\right)(x)=f(T(t) x)$. If $\mathfrak{X}$ is the dual of $\mathfrak{X}_{*}$ and if each $T(t)$ is weak-* continuous, one obtains also a pre-adjoint semigroup $\left\{T(t)_{*}\right\}$ through the same formula; since the weak-* topology is of much more interest than the weak topology for spaces having a predual, this is usually referred to in the literature as the adjoint semigroup (and of course is the restriction of the adjoint semigroup to $\left.\mathfrak{X}_{*} \subset \mathfrak{X}^{*}\right)$. Weak-* continuity and measurability of $\{T(t)\}$ are equivalent to weak continuity and measurability of $\left\{T(t)_{*}\right\}$, so that in particular they are equivalent to each other at times $t>0$ if $\mathfrak{X}_{*}$ is separable.

The last topic to consider for contraction semigroups is the passage from separate to joint continuity. We summarize the results in the following theorem. 
Theorem 1.4.1 (Joint Continuity of $\mathrm{C}_{0}$-Semigroups).

1. Let $\mathfrak{X}$ be a Banach space and $\{T(t)\}_{t \geq 0}$ a contractive $C_{0}$-semigroup. Then $T(t)(x)$ is jointly continuous in $t$ and $x$; that is, the map $[0, \infty) \times \mathfrak{X} \stackrel{T}{\rightarrow} \mathfrak{X}$ is continuous with respect to the norm topology on $\mathfrak{X}$.

2. Let $\mathfrak{X}$ be a Banach space with separable predual $\mathfrak{X}_{*}$, and $\{T(t)\}_{t \geq 0}$ a weak-* continuous semigroup of weak-* continuous contractions on $\mathfrak{X}$. Then $T(t)(x)$ is jointly weak-* continuous in $t$ and $x$ on bounded subsets of $\mathfrak{X}$. That is, the map $[0, \infty) \times \mathfrak{X}_{1} \stackrel{T}{\rightarrow} \mathfrak{X}_{1}$ is continuous with respect to the weak-* topology on $\mathfrak{X}_{1}$.

3. Let $\mathcal{A}$ be a $W^{*}$-algebra and $\left\{\phi_{t}\right\}_{t \geq 0}$ a $C_{0}$-semigroup of strongly continuous contractions on $\mathcal{A}$. Then $\phi_{t}(a)$ is jointly strongly continuous in $t$ and a at nonzero times. That is, the map $(0, \infty) \times \mathcal{A}_{1} \stackrel{\phi}{\rightarrow} \mathcal{A}_{1}$ is continuous with respect to the strong topology on $\mathcal{A}_{1}$.

Proof.

1. By the triangle inequality and the contractivity of the semigroup, $\|T(s)(y)-T(t)(x)\| \leq\|T(s)(y-x)\|+\|T(s) x-T(t) x\| \leq\|y-x\|+\|T(s)(x)-T(t)(x)\|$ which tends to zero as $(s, y) \rightarrow(t, x)$.

2. By Alaoglu's theorem, $\mathfrak{X}_{1}$ is weak-* compact, and since $\mathfrak{X}_{*}$ is assumed to be separable, another standard result implies that $\mathfrak{X}_{1}$ is weakly metrizable ([Con90] V.5.1). Joint weak-* continuity at $(t, a)$ with $t>0$ is therefore a special case of Theorem 4 in CM70]. Joint weak-* continuity at $(0, a)$ is more complicated to 
establish, but is a consequence of Corollary 3.3 of [Law74]. For the purposes of self-containment, we sketch here the relevant arguments.

(a) If $T$ is a Baire space, $X$ a metric space, and $f: T \times X \rightarrow X$ a separately continuous function, then for each $x \in X$ there exists a dense $G_{\delta}$ subset $T_{0} \subseteq T$ such that, for all $t_{0} \in T_{0}, f$ is jointly continuous at $\left(t_{0}, x\right)$. This standard result appears as Exercise XI.10.11 in Dug66 and as Exercise 7.41 in Roy88. Given $x_{0} \in X$, one defines for each $m, n \in \mathbb{N}$ the closed subset

$$
F_{m, n}=\left\{t \in T \mid \forall x \in B_{1 / m}\left(x_{0}\right): d\left(f(t, x), f\left(t, x_{0}\right)\right)<\frac{1}{m}\right\}
$$

the union of all which is $T$. One then defines the open dense subsets

$$
\mathcal{O}_{m}=\bigcup_{n=1}^{\infty} F_{m, n}^{\circ}
$$

of $T$, and the intersection $T_{0}=\bigcap_{m} \mathcal{O}_{m}$ is therefore also dense by the Baire Category Theorem. The sets are constructed in such a way that $f$ is jointly continuous at $\left(t_{0}, x_{0}\right)$ for all $t_{0} \in T_{0}$.

(b) In the case where $T=[0, \infty)$ and $f$ is additive in the first variable in the sense that $f(t+s, x)=f(t, f(s, x))$ and $f(0, x)=x$, one can conclude further that $f$ is jointly continuous at $(t, x)$ for all $x \in X$ and all $t>0$. This is theorem 4 of [CM70], and is proved as follows: Let $x \in X$ and $t>0$. Choose $T_{0} \subset[0, \infty)$ dense such that $f$ is jointly continuous at all $\left(t_{0}, x\right)$ with $t_{0} \in T_{0}$. Because $T_{0}$ is dense in $[0, \infty)$, it must contain some element 
$t_{0}<t$. Then for $t^{\prime}$ sufficiently close to $t$ we will have $t^{\prime}>\min \left(t_{0}, t-t_{0}\right)$, and can write for each $x^{\prime} \in X$

$$
f\left(t^{\prime}, x^{\prime}\right)=f\left(t-t_{0}, f\left(t_{0}+t^{\prime}-t, x^{\prime}\right)\right)
$$

Now as $t^{\prime} \rightarrow t$ and $x^{\prime} \rightarrow x, f\left(t_{0}+t^{\prime}-t, x^{\prime}\right) \rightarrow f\left(t_{0}, x\right)$ by joint continuity at $\left(t_{0}, x\right)$. It follows that $f\left(t-t_{0}, f\left(t_{0}+t^{\prime}-t, x^{\prime}\right)\right) \rightarrow f\left(t-t_{0}, f\left(t_{0}, x\right)\right)=f(t, x)$ by separate continuity.

(c) To establish joint continuity at points $(0, x)$, we add the assumption that $X$ is compact. For each $x \in X$, let $G$ be an open neighborhood of $x$. For each $y \in X \backslash G$, separate continuity implies $f(t, x) \rightarrow x$ and $f(t, y) \rightarrow y$ as $t \rightarrow 0$. Since $T_{0}$ defined as above is dense, one can therefore find a $t_{0} \in T_{0}$ with the property $f\left(t_{0}, x\right) \neq f\left(t_{0}, y\right)$. Some straightforward calculations then imply that there exist open sets $W_{y} \ni 0, U_{y} \ni x$, and $V_{y} \ni y$ such that $f\left(W_{y} \times U_{y}\right)$ is disjoint from $V_{y}$. As the $V_{y}$ form an open cover of the compact set $X \backslash G$, there exists a finite subcover; taking $W$ and $U$ to be the corresponding finite intersections of the $W_{y}$ and $U_{y}$, one has $(0, x) \in W \times U$ and $f(W \times U) \subset G$.

3. For strong continuity, we follow the same proof, using the fact that $\mathcal{A}_{1}$ is also strongly metrizable ([Bla06] III.2.2.27). Since $\mathcal{A}_{1}$ is not strongly compact, however, we cannot infer joint continuity at $(0, a)$. 


\subsubsection{Completely Positive Semigroups}

So far we have considered semigroups of contractions on Banach spaces. When the Banach space happens to be a $\mathrm{W}^{*}$-algebra, and the contractions happen to be normal completely positive maps, some stronger continuity results hold than are true in the more general setting. Here we note two such results. First, recall that a CPsemigroup was defined by the property of point-weak continuity. It turns out that such a semigroup is automatically point-strongly continuous. This is Theorem 3.1 of [MS10].

Our second continuity result which is specific to completely positive semigroups is an improved statement of joint continuity.

Theorem 1.4.2 (Joint Continuity for CP-Semigroups).

Let $\mathcal{A}$ be a separable $W^{*}$-algebra and $\left\{\phi_{t}\right\}_{t \geq 0}$ a CP-semigroup on $\mathcal{A}$.

1. $\phi_{t}(a)$ is jointlyweakly continuous in $t$ and $a$; that is, the map $[0, \infty) \times \mathcal{A}_{1} \stackrel{\phi}{\rightarrow} \mathcal{A}_{1}$ is continuous with respect to the weak topology on $\mathcal{A}_{1}$.

2. $\phi_{t}(a)$ is jointly strongly continuous in $t$ and $a$; that is, the map $[0, \infty) \times \mathcal{A}_{1} \stackrel{\phi}{\rightarrow} \mathcal{A}_{1}$ is continuous with respect to the strong topology on $\mathcal{A}_{1}$.

Proof.

1. This follows from Theorem 1.4.1, we mention it here in order to observe that a considerably simpler proof is available in this special case, which appears as Proposition 2.23 of [Sel97] and as Proposition 4.1(2) of [MS02]. 
2. This is an improvement on Theorem 1.4.1 because of the joint continuity at time 0 , which we shall need later. Assume that $\mathcal{A} \subset B(H)$, with $H$ separable. Let $t_{n} \rightarrow t$ be a convergent sequence in $[0, \infty)$ and $a_{n} \rightarrow a$ an SOT-convergent sequence in $\mathcal{A}_{1}$. (We can use sequences rather than nets because $\mathcal{A}_{1}$ is SOTmetrizable.) By the first part of this theorem, $\phi_{t_{n}}\left(a_{n}\right) \rightarrow \phi_{t}(a)$ in WOT. Now for any $h \in H$,

$$
\begin{aligned}
\left\|\phi_{t_{n}}\left(a_{n}\right) h-\phi_{t}(a) h\right\|^{2} & =\left\|\phi_{t_{n}}\left(a_{n}\right) h\right\|^{2}-2 \operatorname{Re}\left\langle\phi_{t_{n}}\left(a_{n}\right) h, \phi_{t}(a) h\right\rangle+\left\|\phi_{t}(a) h\right\|^{2} \\
& =\left\langle\phi_{t_{n}}\left(a_{n}\right)^{*} \phi_{t_{n}}\left(a_{n}\right) h, h\right\rangle-2 \operatorname{Re}\left\langle\phi_{t_{n}}\left(a_{n}\right) h, \phi_{t}(a) h\right\rangle+\left\|\phi_{t}(a) h\right\|^{2} \\
& \leq\left\langle\phi_{t_{n}}\left(a_{n}^{*} a_{n}\right) h, h\right\rangle-2 \operatorname{Re}\left\langle\phi_{t_{n}}\left(a_{n}\right) h, \phi_{t}(a) h\right\rangle+\left\|\phi_{t}(a) h\right\|^{2}
\end{aligned}
$$

where we have used the Schwarz inequality for 2-positive maps plus the fact that $a_{n}^{*} a_{n} \rightarrow a^{*} a$ in WOT whenever $a_{n} \rightarrow a$ in SOT. Taking the limsup as $n \rightarrow \infty$, we see that $\phi_{t_{n}}\left(a_{n}\right) \rightarrow \phi_{t}(a)$ in SOT.

This appears as Lemma 4 in VS84 and as Lemma 6.4 in Sha08.

\subsection{Survey of Extant Results}

The first results concerning the existence of dilations for cp-semigroups date from the 1970's and pertain to uniformly continuous semigroups. Recall that a contraction semigroup is uniformly continuous iff its generator is bounded; CE79, preceded in special cases by [GKS76] and [Lin76], showed that the generator of a uniformly continuous CP-semigroup on a $\mathrm{W}^{*}$-algebra must have the form $a \mapsto$ $\Psi(a)+k^{*} a+a k$ for some element $k \in \mathcal{A}$ and completely positive map $\Psi: \mathcal{A} \rightarrow \mathcal{A}$. 
This structure theorem was used by [EL77] to prove that a uniformly continuous CP-semigroup on a $\mathrm{W}^{*}$-algebra has an E-dilation. However, attempts to prove the existence of dilations for point-weakly continuous, or even point-norm continuous CP-semigroups were unsuccessful.

Dilations were shown to exist in special cases (for instance, on semigroups having specific forms, on semigroups satisfying additional hypotheses such as the existence of a faithful normal invariant state, in the case of discrete-time semigroups, or using a weaker sense of the word "dilation") by [Emc78, AFL82, [VS84, [Küm85, and others. However, progress on the general problem required a new insight. This insight was the notion of a product system of Hilbert spaces, developed by Arveson (Arv89a, Arv90a, Arv89b, Arv90b]). We shall say more about product systems in chapter 77, briefly, there is an equivalence of categories between $\mathrm{E}_{0}$-semigroups on $B(H)$ and product systems of Hilbert spaces, so that the problem of constructing $\mathrm{E}_{0}$-dilations reduces in some sense to the problem of building a product system out of a $\mathrm{CP}_{0}$-semigroup. Variants of this strategy were used in [Bha96] and [Sel97] to show that every $\mathrm{CP}_{0}$-semigroup on $B(H)$ has an $\mathrm{E}_{0}$-dilation, a result known as Bhat's theorem, and the corresponding result for separable $\mathrm{W}^{*}$-algebras was established in [Arv03]. Later, the more general notion of a product system of Hilbert modules was introduced, leading to new proofs of these theorems in [BS00] and [MS02]. More recently, product systems have been used to study families of completely positive maps indexed by semigroups other than $[0, \infty)$, with the existence of dilations depending on an additional hypothesis known as strong commutativity ([Sha08]). 
A different approach to dilation theory, standing outside this narrative, was proposed by Jean-Luc Sauvageot in [Sau86], Sau88, and Sau91]. Writing during the nascence of free probability (shortly after the publication of [Voi85], for instance), Sauvageot developed a modified version of the free product appropriate for use in dilation theory. Since the Daniell-Kolmogorov construction (Example 1.3.3) can be built using tensor products, which are the coproduct in the category of commutative unital $\mathrm{C}^{*}$-algebras, and since free products play the corresponding role in the category of unital $\mathrm{C}^{*}$-algebras, this is an attractively functorial way to conceptualize a noncommutative Markov process. Using his version of the free product, Sauvageot proved that every $\mathrm{cp}_{0}$-semigroup on a $\mathrm{C}^{*}$-algebra has a unital $\mathrm{e}_{0}$-dilation. This dilation theorem was then used to solve a Dirichlet problem for $\mathrm{C}^{*}$-algebras, much as classical Brownian motion can be used to solve the classical Dirichlet problem ([Kak45]).

Sauvageot's theorem stands virtually alone in achieving a unital dilation; at some point, all the other dilation strategies mentioned here rely upon the non-unital embedding of $B(H)$ into $B(K)$ for Hilbert spaces $H \subset K$. However, although [Sau86] asserts that his dilation technique can be modified to yield continuous dilations on $\mathrm{W}^{*}$-algebras, little detail is given, and later authors indicate some uncertainty about this modification (e.g. Ska02]). Hence, given a $\mathrm{CP}_{0}$-semigroup, it seems that one may be forced to choose either a unital $\mathrm{e}_{0^{-}}$dilation or a continuous (that is, $\mathrm{E}_{0^{-}}$) dilation. The present thesis will expound Sauvageot's dilation techniques in order to demonstrate the possibility of achieving both objectives together (Theorem [5.4.9). 


\section{CHAPTER 2}

\section{LIBERATION}

\subsection{Introduction}

Free probability theory was introduced by Voiculescu in [Voi85], as a tool to address the free group factor problem. Free probability has since blossomed into its own area of study; its development has been an important success, even though the free group factor problem remains unresolved. Sauvageot's ad hoc modification of free probability, in contrast, does not appear to have inspired further pursuit beyond his first paper. This could be due in part to the relevant free independence property remaining implicit in that paper, appearing only in the midst of the proof of Proposition 1.7.

In this chapter, Sauvageot's version of free independence, which I refer to as liberation (meant to suggest something similar to freeness; not to be confused with Voiculescu's use of the same word in [Voi99]) is studied in its own right. As yet the only nontrivial liberated system I know of is the one originally used by Sauvageot in application to dilation theory. However, I still consider it advantageous to separate this part of the exposition, both (i) to clarify the combinatorial aspects of dilation, in contrast to its algebraic and analytic features, and (ii) to suggest possibilities for further investigation of connections with standard free probability theory. 


\subsection{Background: Free Independence and Joint Moments}

We recall some of the basic notions of free probability, which can be found in references such as [Voi85], [VDN92], and [NS06].

A noncommutative probability space is a pair $(\mathcal{A}, \phi)$ where $\mathcal{A}$ is a unital complex algebra and $\phi: \mathcal{A} \rightarrow \mathbb{C}$ a unital linear functional. Subalgebras $\left\{A_{i}\right\}_{i \in I}$ of $\mathcal{A}$ are said to be freely independent with respect to $\phi$ if $\phi\left(a_{i_{1}} a_{i_{2}} \ldots a_{i_{n}}\right)=0$ whenever

- $i_{1}, \ldots, i_{n}$ are elements of $I$ such that adjacent indices are not equal, i.e. for $k=$ $1, \ldots, n-1$ one has $i_{k} \neq i_{k+1}$; this condition is abbreviated as $i_{1} \neq i_{2} \neq \cdots \neq i_{n}$

- $a_{i_{k}} \in A_{i_{k}}$ for each $k=1, \ldots, n$

- $\phi\left(a_{i_{k}}\right)=0$ for each $k=1, \ldots, n$.

Given noncommutative probability spaces $\left\{\left(A_{i}, \phi_{i}\right)\right\}$, a construction known as the free product of unital algebras yields, in a universal (i.e. minimal) way, a noncommutative probability space $(\mathcal{A}, \phi)$ and injections $f_{i}: A_{i} \rightarrow \mathcal{A}$ satisfying $\phi \circ f_{i}=\phi_{i}$, such that the images $f_{i}\left(A_{i}\right)$ are freely independent with respect to $\phi$. Furthermore, this construction on unital algebras can be "promoted" to a construction on unital *-algebras or $\mathrm{C}^{*}$ algebras; in the latter case it is related to the free product of Hilbert spaces.

One implication of free independence which is essential for our present purposes is that it determines the value of $\phi$ on the subalgebra generated by $\left\{A_{i}\right\}$. Given $i_{1} \neq i_{2} \neq \cdots \neq i_{n}$ and elements $a_{i_{k}} \in A_{i_{k}}$, one can compute the joint moment $\phi\left(a_{i_{1}} \ldots a_{i_{n}}\right)$ as follows: 
- Center each term $a_{i_{k}}$; that is, rewrite it as ${\stackrel{\circ}{a_{i}}}_{i_{k}}+\phi\left(a_{i_{k}}\right) \mathbf{1}$, where we define $\stackrel{\circ}{x}=x-\phi(x) \mathbf{1}$.

- Expand the product $\left({\stackrel{\circ}{a_{1}}}_{i_{1}}+\phi\left(a_{i_{1}}\right) \mathbf{1}\right) \cdots\left({\stackrel{\circ}{a_{i_{n}}}}+\phi\left(a_{i_{n}}\right) \mathbf{1}\right)$, thus obtaining a sum of $2^{n}$ words.

- Simplify by pulling out scalars: rewrite, for instance, ${\stackrel{\circ}{a_{1}}}_{(}\left(\phi\left(a_{i_{2}}\right) \mathbf{1}\right){\stackrel{\circ}{i_{3}}}_{i_{3}}$ as $\phi\left(a_{i_{2}}\right){\stackrel{\circ}{i_{1}}}_{i_{1}}{\stackrel{\circ}{i_{3}}}_{i_{3}}$.

- After simplification, the only remaining word of length $n$ is the centered word $\stackrel{\circ}{a}_{i_{1}} \ldots \stackrel{\circ}{a}_{i_{n}}$. Applying the procedure iteratively to all the smaller words that have been generated, one can rewrite the original word as a sum of many centered words, plus a word of length 0, i.e. a scalar. Since $\phi$ vanishes on centered words and is unital, its value at the original word is therefore whatever scalar is left when this iterative procedure terminates.

Using this outline, one can calculate $\phi\left(a_{i_{1}} \ldots a_{i_{n}}\right)$ whenever $i_{1} \neq i_{2} \neq \cdots \neq i_{n}$. Of course, no generality is lost by this hypothesis, as neighboring terms belonging to the same subalgebra can be combined.

For use in proofs, it will be convenient to formalize the above procedure in terms of a recursive definition. I have not seen such a formalization in the literature, so I present the following.

- We use subset notation to indicate sub-tuples of an ordered tuple; thus, $(1,3) \subset$ $(1,2,3,4,5)$ and $(1,2,3,4,5) \backslash(1,3)=(2,4,5)$. We also use $[n]$ to denote the tuple $(1,2, \ldots, n)$. For a set $S$ we use $S^{\sharp}=\bigcup_{n=1}^{\infty} S^{n}$ to denote the set of all finite ordered tuples from $S$. 
- Given a set $I$ and a tuple $\vec{\iota} \in I^{n}$, the consecutivity tuples of $\vec{\iota}$ are the maximal consecutive sub-tuples of $[n]$ such that $i_{j}$ is the same for all $j$ in such a tuple. For instance, if $\vec{\imath}=(1,2,2,1,1,3,1)$ then the consecutivity tuples are $\vec{c}_{1}=(1)$, $\vec{c}_{2}=(2,3), \vec{c}_{3}=(4,5), \vec{c}_{4}=(6)$, and $\vec{c}_{5}=(7)$. We say that $\vec{\iota}$ is nonstammering if all its consecutivity tuples have length 1 ; this is another way of stating the condition $i_{1} \neq i_{2} \neq \cdots \neq i_{n}$.

- Given a noncommutative probability space $(\mathcal{A}, \phi)$ and a nonempty set $I$, we recursively define two moment functions, the alternating moment function $\mathrm{AM}: \mathcal{A}^{\sharp} \rightarrow \mathbb{C}$ and the general moment function GM $:(I \times \mathcal{A})^{\sharp} \rightarrow \mathbb{C}$.

- In the base case $n=1$ we define $\operatorname{AM}\left(a_{1}\right)=\phi\left(a_{1}\right)$.

- Given $\vec{\iota} \in I^{n}$ with consecutivity tuples $\vec{c}_{1}, \ldots, \vec{c}_{\ell}$, define

$$
\operatorname{GM}(\vec{\iota} ; \vec{a})=\operatorname{AM}\left(\prod_{j \in \vec{c}_{1}} x_{j}, \ldots, \prod_{j \in \vec{c}_{\ell}} x_{j}\right)
$$

Note that this defines a general moment in terms of alternating moments of at most the same length.

- For $n>1$ define

$$
\operatorname{AM}\left(a_{1}, \ldots, a_{n}\right)=\sum_{\vec{j} \subsetneq[n]} \operatorname{GM}\left(\vec{j} ;\left(\stackrel{\circ}{a}_{j_{1}}, \ldots, \stackrel{\circ}{a}_{j_{|\vec{j}|}}\right)\right) \prod_{k \in[n] \backslash \vec{j}} \phi\left(a_{k}\right)
$$

where $|\vec{j}|$ denotes the length of $\vec{j}$. By substituting the above definition of the general moment, we see that this defines an alternating moment in terms of alternating moments of strictly shorter words. 
- Note that $\operatorname{AM}\left(a_{1}, \ldots, a_{n}\right)=0$ whenever $\phi\left(a_{1}\right)=\cdots=\phi\left(a_{n}\right)=0$, as the sum is over proper subsets $\vec{j} \subsetneq[n]$ and hence the product $\prod_{k \in[n] \backslash \vec{j}} \phi\left(a_{k}\right)$ is always nonempty and therefore zero.

- Theorem: Let $\mathcal{A}, \phi, I$ be as above, and $\left\{A_{i}\right\}_{i \in I}$ a $\phi$-freely independent family of unital subalgebras of $\mathcal{A}$. For $n \in \mathbb{N}$ and $(\vec{\imath}, \vec{a}) \in(I \times \mathcal{A})^{n}$, we say that $\vec{\iota}$ locates $\vec{a}$ if $a_{k} \in A_{i_{k}}$ for each $k=1, \ldots, n$. Let $\mathcal{A}_{\text {loc }} \subset(I \times \mathcal{A})^{\sharp}$ consist of all $(\vec{\imath}, \vec{a})$ such that $\vec{\iota}$ locates $\vec{a}$, and $\mathcal{A}_{\text {ns }} \subset \mathcal{A}^{\sharp}$ consist of all $\vec{a}$ such that there exists a nonstammering tuple which locates $\vec{a}$. Then

1. for any $(\vec{\imath}, \vec{a}) \in \mathcal{A}_{\mathrm{loc}}$,

$$
\phi\left(a_{1} \ldots a_{n}\right)=\operatorname{GM}(\vec{\imath} ; \vec{a}) .
$$

2. for any $\vec{a} \in \mathcal{A}_{\mathrm{ns}}$,

$$
\phi\left(a_{1} \ldots a_{n}\right)=\operatorname{AM}(\vec{a}) .
$$

The proof is by induction on $n$; using the center-expand-simplify procedure outlined above, one can see that $\phi\left(a_{1} \cdots a_{n}\right)$ satisfies the same recurrence and initial conditions as (the relevant restrictions of) the functions GM and AM.

\subsection{Defining Liberation}

We now develop two variations on free independence, which will be of use in dilation theory.

Definition 2.3.1. Let $\mathcal{C}$ be a unital algebra, $\nu: \mathcal{C} \rightarrow \mathbb{C}$ a unital linear functional, $\mathfrak{e}: \mathcal{C} \rightarrow \mathcal{C}$ a linear map. Given a triple $(A, B, \rho)$ consisting of unital subalgebras 
$A, B \subseteq \mathcal{C}$ and a unital linear map $\rho: A \rightarrow B$ between them satisfying $\mathfrak{e} \circ \rho=\mathfrak{e}$, we introduce the notation $\stackrel{\circ}{a}=a-\rho(a)$ for elements $a \in A$; note that in general $\stackrel{\circ}{a}$ is neither an element of $A$ nor of $B$. We say the triple $(A, B, \rho)$ is:

1. right-liberated (with respect to $\nu$ and $\mathfrak{e}$ ) if $\mathfrak{e}$ is a $B$-bimodule map, i.e. $\mathfrak{e}\left[b_{1} x b_{2}\right]=b_{1} \mathfrak{e}[x] b_{2}$ for all $b_{1}, b_{2} \in B$ and $x \in \mathcal{C}$, and for every $n \geq 1$, every $a_{1}, \ldots, a_{n} \in A$, and every $b_{1}, \ldots, b_{n-1} \in B$ satisfying $\nu\left(b_{1}\right)=\cdots=\nu\left(b_{n-1}\right)=0$,

$$
\mathfrak{e}\left[\stackrel{\circ}{a}_{1} b_{1} \ldots \stackrel{\circ}{a}_{n}\right]=0
$$

2. left-liberated if $\mathfrak{e}$ is an $A$-bimodule map and for every $n \geq 1$, every $a_{1}, \ldots, a_{n-1} \in$ $A$, and every $b_{1}, \ldots, b_{n} \in B$ satisfying $\nu\left(b_{0}\right)=\cdots=\nu\left(b_{n}\right)=0$,

$$
\mathfrak{e}\left[b_{1} \stackrel{\circ}{a}_{1} b_{2} \ldots \stackrel{\circ}{a}_{n-1} b_{n}\right]=0
$$

We note that the criteria in these definitions resemble free independence, in that the alternating product of centered terms is centered. The key difference, however, is that the centering takes place with respect to several different maps - elements of $B$ are centered with respect to $\nu$, elements of $A$ with respect to $\rho$, and the alternating product with respect to $\mathfrak{e}$.

In some cases it will be useful to generalize this definition.

Definition 2.3.2. Let $A, B$ be unital algebras and $\rho: A \rightarrow B$ a unital linear map. A right-liberating representation of the triple $(A, B, \rho)$ is a quintuple $(\mathcal{A}, f, g, \mathfrak{e}, \nu)$ where

- $\mathcal{A}$ is a unital algebra 
- $f: A \rightarrow \mathcal{C}$ and $g: B \rightarrow \mathcal{A}$ are unital homomorphisms

- $\nu: \mathcal{A} \rightarrow \mathbb{C}$ is a unital linear functional

- $\mathfrak{e}: \mathcal{A} \rightarrow \mathcal{A}$ is a (not necessarily unital) linear map

satisfying the following criteria:

1. $\mathfrak{e} \circ g \circ \rho=\mathfrak{e} \circ f$

2. $\mathfrak{e}$ is a $g(B)$-bimodule map

3. for every $n \geq 1$, every and $a_{1}, \ldots, a_{n} \in A$, and every $b_{1}, \ldots b_{n-1} \in B$ such that

$$
\begin{aligned}
\nu\left(g\left(b_{1}\right)\right) & =\ldots \nu\left(g\left(b_{n-1}\right)\right)=0, \\
& \mathfrak{e}\left[\left(f\left(a_{1}\right)-g\left(\rho\left(a_{1}\right)\right)\right) g\left(b_{1}\right) \cdots g\left(b_{n-1}\right)\left(f\left(a_{n}\right)-g\left(\rho\left(a_{n}\right)\right)\right)\right]=0
\end{aligned}
$$

and a left-liberating representation is such a quintuple satisfying

1. $\mathfrak{e} \circ g \circ \rho=\mathfrak{e} \circ f$

2. $\mathfrak{e}$ is an $f(A)$-bimodule map

3. for every $n \geq 1$, every and $a_{1}, \ldots, a_{n-1} \in A$, and every $b_{1}, \ldots b_{n} \in B$ such that

$$
\begin{aligned}
\nu\left(g\left(b_{1}\right)\right) & =\ldots \nu\left(g\left(b_{n}\right)\right)=0, \\
& \mathfrak{e}\left[g\left(b_{1}\right)\left(f\left(a_{1}\right)-g\left(\rho\left(a_{1}\right)\right)\right) \cdots\left(f\left(a_{n-1}\right)-g\left(\rho\left(a_{n-1}\right)\right)\right) g\left(b_{n}\right)\right]=0 .
\end{aligned}
$$

Remark 2.3.3. If there exists a map $\tilde{\rho}: f(A) \rightarrow g(B)$ with the property $\tilde{\rho} \circ f=g \circ \rho$, then Definition (2.3.2) reduces to the statement that $(f(A), g(B), \tilde{\rho})$ is liberated in the appropriate sense from Definition (2.3.1). Such a map $\tilde{\rho}$ need not exist in general, but it does in two important special cases: 
1. If $f$ is injective, one may define $\tilde{\rho}=g \circ \rho \circ f^{-1}$.

2. If $(\mathfrak{e} \circ g)$ is injective, one may define $\tilde{\rho}=g \circ(\mathfrak{e} \circ g)^{-1} \circ \mathfrak{e}$. Then

$$
\tilde{\rho} \circ f=g \circ(\mathfrak{e} \circ g)^{-1} \circ \mathfrak{e} \circ f=g \circ(\mathfrak{e} \circ g)^{-1} \circ \mathfrak{e} \circ g \circ \rho=g \circ \rho .
$$

Both of these cases will be used subsequently.

\subsection{Right Liberation and Joint Moments}

Like free independence, liberation is a property that implies an algorithm. The idea is the same - by centering, expanding, and simplifying, one can write any word as a centered word plus shorter words - but since the centering takes place with respect to three different maps, the details of the procedure are more complicated.

Suppose $\mathcal{C}$ is an algebra in which $(A, B, \rho)$ is right-liberated with respect to $\mathfrak{e}, \nu$ as above. We continue to use the notation $\stackrel{\circ}{a}=a-\rho(a)$ for $a \in A$. Let $\langle A, B\rangle$ denote the subalgebra of $\mathcal{C}$ generated by $A$ and $B$. We consider two types of words in $\langle A, B\rangle$ :

1. A word of the first type is of the form $b_{0} a_{1} b_{1} \ldots b_{\ell-1} a_{\ell} b_{\ell}$ for some $a_{1}, \ldots, a_{\ell} \in A$ and $b_{0}, \ldots, b_{\ell} \in B$.

2. A word of the second type is of the form $b_{0} \stackrel{\circ}{a}_{1} b_{1} \ldots b_{\ell-1} \stackrel{\circ}{a}_{\ell} b_{\ell}$ for some $a_{1}, \ldots, a_{\ell} \in$ $A$ and $b_{0}, \ldots, b_{\ell} \in B$.

Since $B$ is unital (so that we can take $b_{0}=\mathbf{1}$ and/or $b_{\ell}=\mathbf{1}$ ), words of the first type span $\langle A, B\rangle$. However, we will have use for words of both types. We refer to the number $\ell$ above as the length of the word; hence a word of length zero is 
simply an element of $B$. We say that a word of either type is in standard form if $\nu\left(b_{1}\right)=\cdots=\nu\left(b_{\ell-1}\right)=0$.

To calculate $\mathfrak{e}$ on a word $b_{0} a_{1} \cdots a_{\ell} b_{\ell}$ of first type, we proceed thus:

- Center the $b_{i}$ for $0<i<\ell$, expand, and simplify. Here "center" means to write $b_{i}$ as $\tilde{b}_{i}+\nu\left(b_{i}\right) \mathbf{1}$.

The result of this step is a sum of standard-form words of the first type, each with length at most $\ell$. The lengths of some words are less, because $a_{i}\left(\nu\left(b_{i}\right) \mathbf{1}\right) a_{i+1}$ is an element of $A$.

- For each of the resulting words, center the $a_{i}$, expand, and simplify. Here "center" means to write $a_{i}$ as $\stackrel{\circ}{a}_{i}+\rho\left(a_{i}\right)$. Simplification can result in shorter words because $b_{i} \rho\left(a_{i+1}\right) b_{i+1}$ is an element of $B$.

The result of this step is a sum of words of the second type. Not all of these words are in standard form, because simplification can create non-centered elements of $B$. However, all words are of length at most $\ell$, and (crucially) the only word of length $\ell$ is in standard form.

- The resulting words which are not in standard form can be rewritten as sums of words of the first type, by un-centering the $\stackrel{\circ}{a}_{i}$ (that is, writing $\stackrel{\circ}{a}_{i}$ as $a_{i}-\rho\left(a_{i}\right)$ ), expanding, and simplifying. In the resulting sum of words of the first type, all have length strictly less than $\ell$.

- By iterating, this procedure allows us to write our original word $b_{0} a_{1} b_{1} \ldots a_{\ell} b_{\ell}$ as a sum of standard-form words of the second type, plus words of length zero, 
which are just elements of $B$. Since $\mathfrak{e}$ vanishes on standard-form words of the second type and is a $B$-bimodule map, this determines $\mathfrak{e}\left[b_{0} a_{1} \ldots a_{\ell} b_{\ell}\right]$ in terms of $\mathfrak{e}[\mathbf{1}]$

As in the case of ordinary free independence, we can parlay this algorithm into a recursive expression for joint moments. As the procedure is more complicated, however, we end up defining three "moment functions" rather than two.

- Given a noncommutative probability space $(\mathcal{A}, \nu)$, subalgebras $A, B \subset \mathcal{A}$, and a linear map $\rho: A \rightarrow B$, let

$$
\mathcal{W}_{\ell}=\left\{\left(b_{0}, a_{1}, b_{1}, \ldots, a_{\ell}, b_{\ell}\right) \mid a_{1}, \ldots, a_{\ell} \in A ; b_{0}, \ldots, b_{\ell} \in B\right\}, \quad \ell \geq 0
$$

denote the alternating tuples of length $2 \ell+1$ which start and end with an element of $B$, and $\mathcal{W}_{I}=\bigcup_{\ell=0}^{\infty} \mathcal{W}_{\ell}$. This corresponds to the set of type I words as described above. More precisely, if we define the product function $\Pi: \mathcal{W}_{I} \rightarrow \mathcal{A}$ by $\Pi\left(b_{0}, a_{1}, b_{1}, \ldots, a_{\ell}, b_{\ell}\right)=b_{0} a_{1} b_{1} \cdots a_{\ell} b_{\ell}$, then its range $\Pi\left(\mathcal{W}_{I}\right)$ is the set of type I words.

- Given $\ell \geq 1, \vec{x} \in \mathcal{W}_{\ell}$, and a subset $\vec{\iota} \subset[\ell-1]$, we define the left collapse of $\vec{x}$ determined by $\vec{\iota}$, denoted $\operatorname{LC}(\vec{x} ; \vec{\iota})$, as follows: Let $\vec{\iota}=\left(i_{1}, \ldots, i_{m}\right)$. For each $k=1, \ldots, m+1$ define $\alpha_{k}=\prod_{j=i_{k-1}+1}^{i_{k}} a_{j}$, where we adopt the convention $i_{0}=0$ and $i_{m+1}=\ell$.

Then $\operatorname{LC}(\vec{x} ; \vec{\iota})=\left(b_{0}, \alpha_{1}, b_{i_{1}}-\nu\left(b_{i_{1}}\right) \mathbf{1}, \ldots, \alpha_{m}, b_{i_{m}}-\nu\left(b_{i_{m}}\right) \mathbf{1}, \alpha_{m+1}, b_{\ell}\right)$. This is the vector that results by taking $\vec{x}$, replacing those $b_{j}$ with $0<j<\ell$ and $j \in \vec{\imath}$ 
by $b_{j}-\nu\left(b_{j}\right) \mathbf{1}$, replacing those $b_{j}$ with $0<j<\ell$ and $j \notin \vec{\imath}$ by $\mathbf{1}$, and then multiplying together adjacent terms from $A$. It is an element of $\mathcal{W}_{|\vec{\imath}|+1}$.

- Similarly, given $\vec{x} \in \mathcal{W}_{\ell}$ and a subset $\vec{\iota} \subset[\ell]$, we define the right collapse of $\vec{x}$ determined by $\vec{\iota}$, an element of $\mathcal{W}_{|\vec{\iota}|}$ denoted $\operatorname{RC}(\vec{x} ; \vec{\iota})$, as follows: Let $\vec{\iota}=\left(i_{1}, \ldots, i_{m}\right)$. For each $k=0, \ldots, m$ define $\beta_{k}=b_{i_{k}} \prod_{j=i_{k}+1}^{i_{k+1}-1} \rho\left(a_{j}\right) b_{j}$, where we retain the convention $i_{0}=0$ but now set $i_{m+1}=\ell+1$. Then $\operatorname{RC}(\vec{x} ; \vec{\iota})=$ $\left(\beta_{0}, a_{i_{1}}, \beta_{1}, \ldots, a_{i_{m}}, \beta_{m}\right)$. This is the vector that results by taking $\vec{x}$, replacing those $a_{j}$ with $j \notin \vec{\imath}$ by $\rho\left(a_{j}\right)$, and then multiplying together adjacent terms from $B$.

- Given $\vec{y} \in \mathcal{W}_{\ell}$ and a subset $\vec{\iota} \subset[\ell]$, we define the un-collapse of $\vec{x}$ determined by $\vec{\iota}$, an element of $\mathcal{W}_{|\vec{\iota}|}$ denoted $\mathrm{UC}(\vec{x} ; \vec{\imath})$, as follows: Let $\vec{\iota}=\left(i_{1}, \ldots, i_{m}\right)$. For each $k=0, \ldots, m$ define $\beta_{k}=b_{i_{k}} \prod_{j=i_{k}+1}^{i_{k+1}-1}\left(-\rho\left(a_{j}\right)\right) b_{j}$, where we continue to interpret $i_{0}$ as 0 and $i_{m+1}$ as $\ell+1$. Then $\operatorname{UC}(\vec{x} ; \vec{\iota})=\left(\beta_{0}, a_{i_{1}}, \beta_{1}, \ldots, a_{i_{m}}, \beta_{m}\right)$. This is the vector that results by taking $\left(b_{0}, a_{1}, b_{1}, \ldots, a_{\ell}, b_{\ell}\right)$, replacing each $a_{j}$ with $j \notin \vec{\imath}$ by $-\rho\left(a_{j}\right)$, and then multiplying together adjacent terms from $B$.

- We define three functions LM, RM, UM $: \mathcal{W}_{I} \rightarrow B$, which we call the leftcentering moment function, the right-centering moment function, and the un- 
centering moment function, by

$$
\begin{aligned}
\operatorname{LM}\left(b_{0}\right) & =\operatorname{RM}\left(b_{0}\right)=\mathrm{UM}\left(b_{0}\right)=b_{0}, \\
\operatorname{LM}(\vec{x}) & =\sum_{\vec{\iota} \subseteq[\ell]} \operatorname{RM}(\operatorname{LC}(\vec{x} ; \vec{\iota})) \prod_{j \in[\ell] \backslash \vec{\iota}} \nu\left(x_{2 j+1}\right), \\
\operatorname{RM}(\vec{x}) & =\sum_{\vec{\iota} \subsetneq[\ell+1]} \operatorname{UM}(\operatorname{RC}(\vec{x} ; \vec{\iota})), \\
\mathrm{UM}(\vec{x}) & =\sum_{\vec{\imath} \subseteq[\ell+1]} \operatorname{LM}(\mathrm{UC}(\vec{x} ; \vec{\imath}))
\end{aligned}
$$

for $\vec{x} \in \mathcal{W}_{\ell+1}$. This produces a well-defined recursion because of the strict subset inclusion $\vec{\iota} \subsetneq[\ell+1]$ in the definition of RM, so that one obtains moments of strictly shorter words.

Remark 2.4.1. Note that evaluating the LM function on a word of length $\ell$ returns a sum of $2^{\ell-1}$ evaluations of the RM function on words of length up to $\ell$; on a word of size $k \leq \ell$, the RM function returns a sum of $2^{k-1}$ evaluations of the UM function on words of length strictly less than $k$; and on a word of size $j<k$, the UM function returns a sum of $2^{j}$ evaluations of the LM function on words of length up to $j$. This implies that the number of terms in the evaluation of the LM on words of length $\ell$ is bounded above by the sequence $\left\{s_{\ell}\right\}$ determined by $s_{0}=1$ and $s_{\ell+1}=8^{\ell+1} s_{\ell}$, which has the closed form $s_{\ell}=8^{\ell(\ell+1) / 2}$. Of course the actual number of terms is considerably less, due both to cancellation and to the fact that this estimate treats all words of length less than $\ell$ as if they had length $\ell$.

Theorem 2.4.2. Let $(A, B, \rho)$ be right-liberated in $\mathcal{A}$ with respect to $\mathfrak{e}, \nu$. Then for 
any $\vec{x} \in \mathcal{W}_{I}$,

$$
\mathfrak{e}[\Pi(\vec{x})]=\operatorname{LM}(\vec{x}) \mathfrak{e}[\mathbf{1}]
$$

Proof. We prove this for $\vec{x} \in \mathcal{A}_{\ell}$ by induction on $\ell$. The base case $\ell=0$ is trivial, and in general, the center-expand-simplify procedure shows that $\mathfrak{e}[\Pi(\vec{x})]$ satisfies the same recursion as $\operatorname{LM}(\vec{x}) \mathfrak{e}[\mathbf{1}]$. The strict subset inclusion in the definition of $\mathrm{RM}$ arises because the $\vec{\iota}=[\ell]$ term corresponds to a centered word, which vanishes when $\mathfrak{e}$ is applied.

Corollary 2.4.3. Let $(A, B, \rho)$ be right-liberated in $\mathcal{A}$ with respect to $\mathfrak{e}, \nu$. Let $\langle A, B\rangle$ denote the subalgebra of $\mathcal{A}$ generated by $A$ and $B$. Then

$$
\mathfrak{e}[\langle A, B\rangle]=\mathfrak{e}[B]
$$

The obvious generalizations of Theorem (2.4.2) and Corollary (2.4.3) to rightliberating representations is true as well, and are verified inductively in the same manner. We record them here without proof.

Theorem 2.4.4. Let $(\mathcal{A}, f, g, \mathfrak{e}, \nu)$ be a right-liberating representation of $(A, B, \rho)$. For $\vec{x}=\left(b_{0}, a_{1}, b_{1}, \ldots, a_{\ell}, b_{\ell}\right) \in \mathcal{W}_{I}$, let $(f \times g)(\vec{x})$ denote the element $g\left(b_{0}\right) f\left(a_{1}\right) g\left(b_{1}\right) \ldots f\left(a_{\ell}\right) g\left(b_{\ell}\right) \in \mathcal{A}$. Define the functions $\mathrm{LM}_{r}, \mathrm{RM}_{r}, \mathrm{UM}_{r}: \mathcal{W}_{I} \rightarrow B$ as in equations (2.1)-2.4, except with $\nu$ replaced by $\nu \circ g$.

Then for any $\vec{x} \in \mathcal{W}_{I}$,

$$
\mathfrak{e}[(f \times g)(\vec{x})]=\operatorname{LM}_{r}(\vec{x}) \mathfrak{e}[\mathbf{1}]
$$


Corollary 2.4.5. Let $(\mathcal{A}, f, g, \mathfrak{e}, \nu)$ be a right-liberating representation of $(A, B, \rho)$. Let $\langle A, B\rangle$ denote the subalgebra of $\mathcal{A}$ generated by $f(A)$ and $g(B)$. Then

$$
\mathfrak{e}[\langle A, B\rangle]=\mathfrak{e}[g(B)]
$$

Later we shall be interested in the continuity properties of joint moments. We record here the following simple observation:

Proposition 2.4.6. Let $(A, B, \rho)$ be right-liberated in $\mathcal{A}$ with respect to $(\mathfrak{e}, \nu)$, where $\mathcal{A}$ is a $W^{*}$-algebra and $\rho, \mathfrak{e}, \nu$ are all normal. Then

1. For any $\vec{x} \in \mathcal{W}_{I}, \operatorname{LM}(\vec{x})$ is normal in each entry of $x$. That is, given $\ell \geq 0$, $\vec{x} \in \mathcal{W}_{\ell}$, and $1 \leq j \leq 2 \ell+1$, let $x_{k}$ be fixed for all $1 \leq k \leq 2 \ell+1$ with $k \neq j$; then $\operatorname{LM}(\vec{x})$, viewed as a function of $x_{j}$, is a normal linear map from $A$ or $B$ (depending on the parity of $j$ ) to $\mathcal{A}$.

2. If $\rho$ is strongly continuous on the unit ball $A_{1}$, then $\operatorname{LM}(\vec{x})$ is jointly strongly continuous in the entries of $\vec{x}$ on bounded subsets. That is, the corresponding map $A_{1} \times B_{1} \times \cdots \times A_{1} \rightarrow \mathcal{A}$ is strongly continuous.

The proof is a straightforward induction on $\ell$. For the second part we use the fact that $\mathfrak{e}$ and $\nu$, being completely positive and normal, are therefore also strongly continuous ([Bla06] III.2.2.2), and that multiplication is jointly strongly continuous on the unit ball.

Later we will need to consider moments with respect to several maps. When need arises, we use $\operatorname{LM}(\vec{x} ; \rho)$ in place of $\operatorname{LM}(\vec{x})$ for specificity. 
Proposition 2.4.7. Let $\mathcal{A}$ be a $W^{*}$-algebra, e and $\nu$ normal, $A \subset \mathcal{A}$ a $W^{*}$-subalgebra, and $\left\{\phi_{t}\right\}_{t \geq 0}$ a CP-semigroup on $A$. Then for each fixed $\vec{x} \in \mathcal{W}_{I}, \operatorname{LM}\left(\vec{x} ; \phi_{t}\right)$ is strongly continuous in $t$.

Proof. As discussed in section 1.4.2, $t \mapsto \phi_{t}(a)$ is strongly continuous for fixed $a \in A$. The result is now a straightforward induction, using this fact plus the joint strong continuity of multiplication on the unit ball of $A$.

\subsection{Left Liberation and Joint Moments}

Unlike right liberation and strong right liberation, left liberation is a condition involving an unspecified scalar; as this scalar can propagate through the recursion, calculation of moments is impossible. However, we can still draw some important conclusions.

We use a similar definition of words of the first and second types, but with the roles of $A$ and $B$ interchanged; that is, our new type I words are of the form $a_{0} b_{1} a_{1} \ldots b_{\ell} a_{\ell}$, and our new type II words of the form $a_{0} b_{1} \stackrel{\circ}{1}_{1} b_{2} \stackrel{\circ}{a}_{2} \ldots b_{\ell-1} \stackrel{\circ}{a}_{\ell-1} b_{\ell} a_{\ell}$. A word of either type is in standard form if $\nu\left(b_{1}\right)=\cdots=\nu\left(b_{\ell}\right)=0$.

The new center-expand-simplify strategy is as follows:

- Given a word of type I, center the $b_{i}$, expand, and collapse. The result is a sum of type I words in standard form of length at most $\ell$.

- Center the $a_{i}$ for $0<i<\ell$, expand, and collapse. The result is a sum of type II words of length at most $\ell$, such that the only word of length $\ell$ is in standard form. 
- For remaining words not in standard form, un-center the $a_{i}$, then apply the same procedure to the type I words that result.

We formalize this strategy in the following definitions:

- For each $\ell \geq 0$ let

$$
\widetilde{\mathcal{W}}_{\ell}=\left\{\left(a_{0}, b_{1}, a_{1}, \ldots, b_{\ell}, a_{\ell}\right) \mid a_{0}, \ldots, a_{\ell} \in A ; b_{1}, \ldots, b_{\ell} \in B\right\}
$$

and let $\widetilde{\mathcal{W}}_{I}=\bigcup_{\ell=0}^{\infty} \widetilde{\mathcal{W}}_{\ell}$. Define the product function $\widetilde{\Pi}: \widetilde{\mathcal{W}}_{I} \rightarrow \mathcal{A}$ by $\widetilde{\Pi}\left(a_{0}, b_{1}, a_{1}, \ldots, b_{\ell}, a_{\ell}\right)=a_{0} b_{1} a_{1} \ldots b_{\ell} a_{\ell}$. Note that the span of $\widetilde{\Pi}\left(\widetilde{\mathcal{W}}_{I}\right)$ is $\langle A, B\rangle$.

- Given $\ell \geq 1, \vec{x} \in \widetilde{\mathcal{W}}_{\ell}$, and a subset $\vec{\imath} \subset[\ell]$, suppose that $\vec{\imath}=\left(i_{1}, \ldots, i_{m}\right)$. For ecah $k=0, \ldots, m$ define $\alpha_{k}=\prod_{j=i_{k}}^{i_{k+1}-1} a_{j}$, with the conventions $i_{0}=0$ and $i_{m+1}=\ell+1$. Then we define

$$
\mathrm{LC}^{\prime}(\vec{x} ; \vec{\iota})=\left(\alpha_{0}, b_{i_{1}}-\nu\left(b_{i_{1}}\right) \mathbf{1}, \alpha_{1}, \ldots, b_{i_{m}}-\nu\left(b_{i_{m}}\right) \mathbf{1}, \alpha_{m}\right)
$$

This is the vector that results by starting with $\vec{x}$, replacing those $b_{j}$ with $j \in \vec{\imath}$ by $b_{j}-\nu\left(b_{j}\right) \mathbf{1}$, and the others by $\mathbf{1}$, and multiplying together adjacent terms from $A$. It is an element of $\widetilde{\mathcal{W}}_{|\vec{\iota}|}$.

- Given $\vec{x} \in \widetilde{\mathcal{W}}_{\ell}$ and a subset $\vec{\iota} \subset[\ell-1]$, suppose $\vec{\iota}=\left(i_{1}, \ldots, i_{m}\right)$. For each $k=1, \ldots, m+1$ let $\beta_{k}=b_{i_{k}} \prod_{j=i_{k}}^{i_{k+1}-1} \rho\left(a_{j}\right) b_{j+1}$, and define

$$
\mathrm{RC}^{\prime}(\vec{x} ; \vec{\iota})=\left(a_{0}, \beta_{1}, a_{i_{1}}, \ldots, a_{i_{m}}, \beta_{m}, a_{\ell}\right)
$$

This is the vector that results from starting with $\vec{x}$, replacing those $a_{j}$ with $j \notin \vec{\iota}$ by $\rho\left(a_{j}\right)$, and then multiplying together adjacent terms from $B$. It is an element of $\widetilde{\mathcal{W}}_{|\vec{\imath}|+1}$. 
- As before, the un-collapse is defined in the same way except with a minus sign; that is, redefine $\beta_{k}=b_{i_{k}} \prod_{j=i_{k}}^{i_{k+1}-1}\left(-\rho\left(a_{j}\right)\right) b_{j+1}$ and then let

$$
\mathrm{UC}^{\prime}(\vec{x} ; \vec{\iota})=\left(a_{0}, \beta_{1}, a_{i_{1}}, \ldots, a_{i_{m}}, \beta_{m}, a_{\ell}\right) .
$$

- Define $\mathrm{LM}^{\prime}, \mathrm{RM}^{\prime}, \mathrm{UM}^{\prime}: \widetilde{\mathcal{W}}_{I} \rightarrow A$ by

$$
\begin{aligned}
\operatorname{LM}^{\prime}\left(a_{0}\right) & =\operatorname{RM}^{\prime}\left(a_{0}\right)=\operatorname{UM}^{\prime}\left(a_{0}\right)=a_{0}, \\
\operatorname{LM}^{\prime}(\vec{x}) & =\sum_{\vec{\iota} \subset[\ell+1]} \operatorname{RM}^{\prime}\left(\operatorname{LC}^{\prime}(\vec{x} ; \iota)\right) \prod_{j \in[\ell+1] \backslash \vec{\iota}} \nu\left(x_{2 j}\right), \\
\operatorname{RM}^{\prime}(\vec{x}) & =\sum_{\vec{\imath} \succ[\ell]} \operatorname{UM}^{\prime}\left(\operatorname{RC}^{\prime}(\vec{x} ; \vec{\iota})\right), \\
\operatorname{UM}^{\prime}(\vec{x}) & =\sum_{\vec{\imath} \succ[\ell]} \operatorname{LM}^{\prime}\left(\operatorname{UC}^{\prime}(\vec{x} ; \vec{\iota})\right)
\end{aligned}
$$

for $\vec{x} \in \widetilde{\mathcal{W}}_{\ell+1}$.

We then have the following analogues of our prior results.

Theorem 2.5.1. Let $(A, B, \rho)$ be left-liberated in $\mathcal{A}$ with respect to $\mathfrak{e}, \nu$. Then for any $\vec{x} \in \widetilde{\mathcal{W}}_{I}$,

$$
\mathfrak{e}[\widetilde{\Pi}(\vec{x})]=\operatorname{LM}^{\prime}(\vec{x}) \mathfrak{e}[\mathbf{1}]
$$

Proof. The center-expand-simplify procedure shows that $\mathfrak{e}[\widetilde{\Pi}(\vec{x})]$ satisfies the same recursion as $\operatorname{LM}^{\prime}(\vec{x}) \mathfrak{e}[\mathbf{1}]$.

Corollary 2.5.2. Let $(A, B, \rho)$ be left-liberated in $\mathcal{A}$ with respect to $\mathfrak{e}, \nu$. Let $\langle A, B\rangle$ denote the subalgebra of $\mathcal{A}$ generated by $A$ and $B$. Then

$$
\mathfrak{e}[\langle A, B\rangle]=\mathfrak{e}[A]
$$


Theorem 2.5.3. Let $(\mathcal{A}, f, g, \mathfrak{e}, \nu)$ be a left-liberating representation of $(A, B, \rho)$. For $\vec{x}=\left(a_{0}, b_{1}, a_{1}, \ldots, b_{\ell}, a_{\ell}\right) \in \mathcal{W}_{I}$, let $(f \times g)(\vec{x})$ denote the element $f\left(a_{0}\right) g\left(b_{1}\right) f\left(a_{1}\right) \ldots g\left(b_{\ell}\right) f\left(a_{\ell}\right) \in \mathcal{A}$. Define the functions $\mathrm{LM}_{r}^{\prime}, \mathrm{RM}_{r}^{\prime}, \mathrm{UM}_{r}^{\prime}: \mathcal{W}_{I} \rightarrow B$ as in equations (2.5)-(2.8), except with $\nu$ replaced by $\nu \circ g$.

Then for any $\vec{x} \in \widetilde{\mathcal{W}}_{I}$,

$$
\mathfrak{e}[(f \times g)(\vec{x})]=\operatorname{LM}_{r}^{\prime}(\vec{x}) \mathfrak{e}[\mathbf{1}] .
$$

Corollary 2.5.4. Let $(\mathcal{A}, f, g, \mathfrak{e}, \nu)$ be a left-liberating representation of $(A, B, \rho)$. Let $\langle A, B\rangle$ denote the subalgebra of $\mathcal{A}$ generated by $f(A)$ and $g(B)$. Then

$$
\mathfrak{e}[\langle A, B\rangle]=\mathfrak{e}[f(A)]
$$




\section{CHAPTER 3}

\section{THE SAUVAGEOT PRODUCT}

\subsection{Introduction}

In this chapter we develop a modification of the unital free product of $\mathrm{C}^{*}$ algebras, adapted for use in dilation theory. As mentioned in example (1.3.3), the classical Daniell-Kolmogorov construction can be reduced to the construction of maps $\theta_{t}: C(S) \otimes C(S) \rightarrow C(S)$ given on simple tensors by $\theta_{t}(f \otimes g)=\left(P_{t} f\right) g$. We shall return to the details of this reduction in chapter 4; at present we only describe enough of its features to see what we shall need for the appropriate noncommutative analogue.

Among the many embeddings of $C(S)$ into $C(S) \otimes C(S)$ we distinguish two, the "left" embedding $f \mapsto f \otimes \mathbf{1}$ and the "right" embedding $f \mapsto \mathbf{1} \otimes f$. The map $\theta_{t}$ is a retraction with respect to the right embedding, and its composition with the left embedding is $P_{t}$. That is, by constructing $\theta_{t}$ we factor $P_{t}$ into an embedding followed by a retraction (with respect to a different embedding), as depicted in the following diagram:

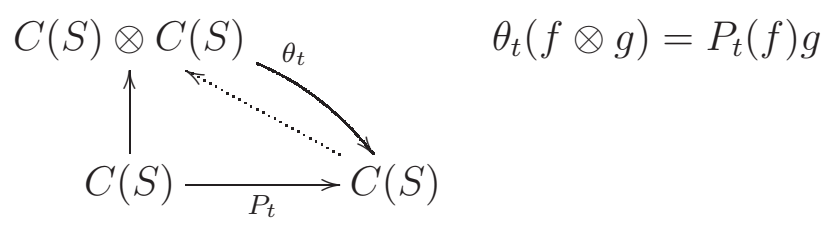

More generally, the inductive process will work with tensor powers $C(S)^{\otimes \gamma}$ for finite sets $\gamma \subset[0, \infty)$, building for each one a retraction $\epsilon_{\gamma}: C(S)^{\otimes \gamma} \rightarrow C(S)$. Given 
$\gamma^{\prime}=\gamma \cup\left\{t_{k}\right\}$, where $\tau=t_{k}-\min _{t \in \gamma} t>0$, we will seek to define $\epsilon_{\gamma^{\prime}}$ such that

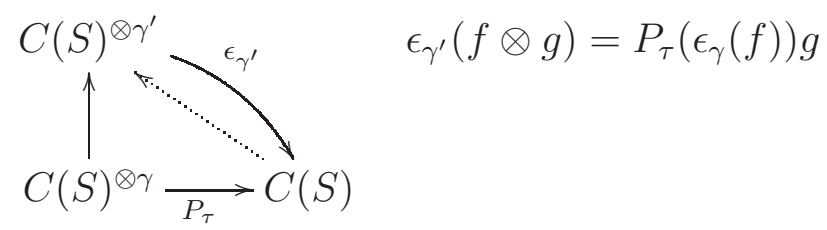

We note in passing that Stinespring dilation produces a very similar diagram: Given a unital completely map $\phi: A \rightarrow B(H)$ with minimal Stinespring triple $(K, V, \pi)$, we obtain

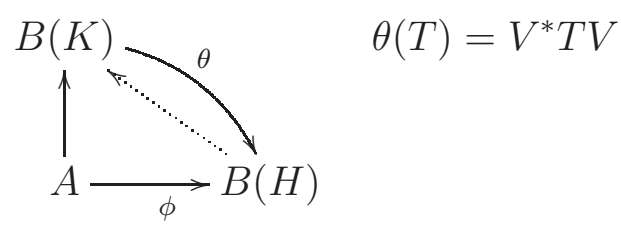

Crucially, however, the right embedding in this case is the non-unital map $X \mapsto$ $V X V^{*}$, in contrast to the unital embedding in the commutative example. Hence, we take the tensor product as our model in what follows.

In addition to constructing tensor products $C(X) \otimes C(Y) \simeq C(X \times Y)$ of commutative unital $\mathrm{C}^{*}$-algebras, one can also form tensor products of maps between them, and the resulting maps satisfy certain functorial properties. We summarize the properties of the tensor product which we shall seek to replicate in this chapter:

1. Given unital $\mathrm{C}^{*}$-algebras $A, B$ and a unital completely positive map $A \stackrel{\phi}{\rightarrow} B$, we construct a unital $\mathrm{C}^{*}$-algebra $A \star B$ with unital embeddings of $A$ and $B$, the images of which generate $A \star B$.

2. We also construct a retraction $A \star B \rightarrow B$ which factors $\phi$ in the sense of the above diagrams. 
3. Given unital completely positive maps $A \stackrel{\phi}{\rightarrow} B$ and $C \stackrel{\psi}{\rightarrow} D$, and given unital *-homomorphisms $A \stackrel{f}{\rightarrow} C$ and $B \stackrel{g}{\rightarrow} D$ such that the square

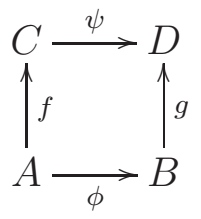

commutes, we construct a (necessarily unique) unital *-homomorphism $f \star g: A \star B \rightarrow C \star D$ such that the squares
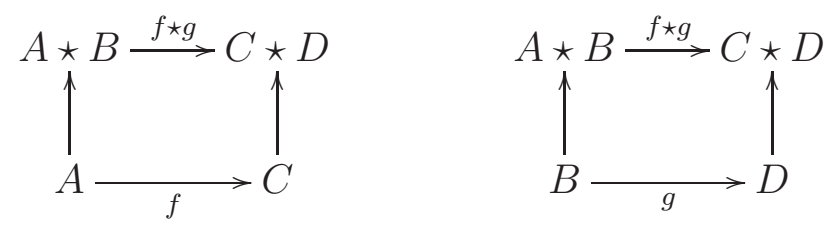

commute.

We now begin our development of a construction satisfying these requirements.

\subsection{Sauvageot Products of Hilbert Spaces and Bounded Operators}

Just as the free product of unital $\mathrm{C}^{*}$-algebras can be constructed from a free product of Hilbert spaces ([Voi85]), our product construction on $\mathrm{C}^{*}$-algebras will rely on an underlying construction on Hilbert space. Some notational preliminaries: For a Hilbert space $H, H^{+}$denotes $H \oplus \mathbb{C}$, and if a unit vector has been distinguished, $H^{-}$denotes the complement of its span. A distinguished unit vector (such as $1 \in \mathbb{C}$ as an element of the direct sum $H \oplus \mathbb{C}$ ) is generally denoted by $\Omega$. We also follow the convention (most common in physics and in Hilbert $\mathrm{C}^{*}$-modules) that inner products are linear in the second variable. 
Definition 3.2.1. Let $\mathcal{H}$ and $\mathcal{L}$ be Hilbert spaces. The Sauvageot product $\mathcal{H} \star \mathcal{L}$ is the space

$$
\mathcal{H} \star \mathcal{L}=\mathcal{H}^{+} \oplus \bigoplus_{n=0}^{\infty}\left[\left(\mathcal{L}^{+\otimes n} \otimes \mathcal{L}\right) \oplus\left(\mathcal{H} \otimes \mathcal{L}^{+\otimes n} \otimes \mathcal{L}\right)\right]
$$

with the convention $\mathcal{L}^{+\otimes 0} \otimes \mathcal{L}=\mathcal{L}$.

Though defined as a direct sum, the Sauvageot product of Hilbert spaces may also be viewed as an infinite tensor product, as expressed in the following proposition.

Proposition 3.2.2. Let $\mathcal{H}$ and $\mathcal{L}$ be Hilbert spaces, and $\mathcal{K}=\mathcal{H}^{+} \oplus \mathcal{L}$. Denote by $\mathcal{L}^{+\otimes \mathbb{N}}$ the infinite tensor power of $\mathcal{L}^{+}$with respect to $\Omega$. Then there are unitary equivalences between $\mathcal{H} \star \mathcal{L}$ and both $\mathcal{H}^{+} \otimes \mathcal{L}^{+\otimes \mathbb{N}}$ and $\mathcal{K} \otimes \mathcal{L}^{+\otimes \mathbb{N}}$, under which

- the subspace $\mathcal{H} \otimes \mathcal{L}^{+\otimes \mathbb{N}}$ of $\mathcal{H}^{+} \otimes \mathcal{L}^{+\otimes \mathbb{N}}$ is identified with the subspace $\bigoplus_{n=0}^{\infty} \mathcal{H} \otimes \mathcal{L}^{+\otimes n} \otimes \mathcal{L}$ of $\mathcal{H} \star \mathcal{L}$

- the subspace $\mathbb{C} \otimes \mathcal{L}^{+\otimes \mathbb{N}}$ of $\mathcal{H}^{+} \otimes \mathcal{L}^{+\otimes \mathbb{N}}$ is identified with the subspace $\bigoplus_{n=0}^{\infty} \mathcal{L}^{+\otimes n} \otimes \mathcal{L}$ of $\mathcal{H} \star \mathcal{L}$

- the subspace $\mathcal{H} \otimes \mathcal{L}^{+\otimes \mathbb{N}}$ of $\mathcal{K} \otimes \mathcal{L}^{+\otimes \mathbb{N}}$ is identified with the subspace $\mathcal{H} \oplus \bigoplus_{n=0}^{\infty} \mathcal{H} \otimes \mathcal{L}^{+\otimes n} \otimes \mathcal{L}$ of $\mathcal{H} \star \mathcal{L}$

- the subspace $\mathbb{C} \otimes \mathcal{L}^{+\otimes \mathbb{N}}$ of $\mathcal{K} \otimes \mathcal{L}^{+\otimes \mathbb{N}}$ is identified with the subspace $\mathcal{L} \oplus \bigoplus_{n=1}^{\infty} \mathbb{C} \otimes \mathcal{L}^{+\otimes(n-1)} \otimes \mathcal{L}$ of $\mathcal{H} \star \mathcal{L}$

- the subspace $\mathcal{L} \otimes \mathcal{L}^{+\otimes \mathbb{N}}$ of $\mathcal{K} \otimes \mathcal{L}^{+\otimes \mathbb{N}}$ is identified with the subspace $\bigoplus_{n=1}^{\infty} \mathcal{L} \otimes \mathcal{L}^{+\otimes(n-1)} \otimes \mathcal{L}$ of $\mathcal{H} \star \mathcal{L}$ 
Proof. We will use the unitary equivalences $\mathcal{L}^{+\otimes \mathbb{N}} \simeq \mathcal{L}^{+} \otimes \mathcal{L}^{+\otimes \mathbb{N}}$, which is evident, and $\mathcal{L}^{+\otimes \mathbb{N}} \simeq \mathbb{C} \oplus \bigoplus_{n=0}^{\infty} \mathcal{L}^{+\otimes n} \otimes \mathcal{L}$, which follows from the construction of the infinite tensor power: If $V_{\infty, n}: \mathcal{L}^{+\otimes n} \rightarrow \mathcal{L}^{+\otimes \mathbb{N}}$ denotes the limit map, then $\mathcal{L}^{+\otimes \mathbb{N}}=\overline{\bigcup_{n=0}^{\infty} V_{\infty, n} \mathcal{L}^{+\otimes n}}$ and in order to get a direct sum we orthogonalize using $\left(V_{\infty, n+1} \mathcal{L}^{+\otimes(n+1)}\right) \ominus\left(V_{\infty, n} \mathcal{L}^{+\otimes n}\right) \simeq \mathcal{L}^{+\otimes n} \otimes \mathcal{L}$. Repeated application of these equivalences plus the associative, commutative, and distributive laws $H \otimes\left(K_{1} \otimes K_{2}\right) \simeq\left(H \otimes K_{1}\right) \otimes K_{2}, \quad H \otimes K \simeq K \otimes H, \quad H \otimes\left(K_{1} \oplus K_{2}\right) \simeq\left(H \otimes K_{1}\right) \oplus\left(H \otimes K_{2}\right)$ and the identity $H \otimes \mathbb{C} \simeq H$ yield

$$
\begin{aligned}
\mathcal{K} \otimes \mathcal{L}^{+\otimes \mathbb{N}} & =\left(\mathcal{H}^{+} \oplus \mathcal{L}\right) \otimes \mathcal{L}^{+\otimes \mathbb{N}} \simeq\left(\mathcal{H} \oplus \mathcal{L}^{+}\right) \otimes \mathcal{L}^{+\otimes \mathbb{N}} \simeq\left(\mathcal{H} \otimes \mathcal{L}^{+\otimes \mathbb{N}}\right) \oplus\left(\mathcal{L}^{+} \otimes \mathcal{L}^{+\otimes \mathbb{N}}\right) \\
& \simeq\left(\mathcal{H} \otimes \mathcal{L}^{+\otimes \mathbb{N}}\right) \oplus \mathcal{L}^{+\otimes \mathbb{N}} \simeq\left(\mathcal{H} \otimes \mathcal{L}^{+\otimes \mathbb{N}}\right) \oplus\left(\mathbb{C} \otimes \mathcal{L}^{+\otimes \mathbb{N}}\right) \simeq \mathcal{H}^{+} \otimes \mathcal{L}^{+\otimes \mathbb{N}}
\end{aligned}
$$

and

$$
\begin{aligned}
\mathcal{H} \star \mathcal{L} & =\mathcal{H}^{+} \oplus \bigoplus_{n=0}^{\infty}\left(\mathcal{H} \otimes \mathcal{L}^{+\otimes n} \otimes \mathcal{L} \oplus \mathcal{L}^{+\otimes n} \otimes \mathcal{L}\right) \\
& \simeq\left(\mathcal{H}^{+} \otimes \mathbb{C}\right) \oplus \bigoplus_{n=0}^{\infty}\left(\mathcal{H} \otimes \mathcal{L}^{+\otimes n} \otimes \mathcal{L} \oplus \mathbb{C} \otimes \mathcal{L}^{+\otimes n} \otimes \mathcal{L}\right) \\
& \simeq\left(\mathcal{H}^{+} \otimes \mathbb{C}\right) \oplus \bigoplus_{n=0}^{\infty}\left(\mathcal{H}^{+} \otimes \mathcal{L}^{+\otimes n} \otimes \mathcal{L}\right) \simeq \mathcal{H}^{+} \otimes\left(\mathbb{C} \oplus \bigoplus_{n=0}^{\infty} \mathcal{L}^{+\otimes n} \otimes \mathcal{L}\right) \simeq \mathcal{H}^{+} \otimes \mathcal{L}^{+\otimes \mathbb{N}}
\end{aligned}
$$

The specific identifications arise by following subspaces through these equivalences.

As a simple corollary, we obtain the folllowing identifications:

Proposition 3.2.3. Let $\mathcal{H}, \mathcal{H}_{1}, \mathcal{H}_{2}, \mathcal{L}$ be Hilbert spaces.

1. $\left(\mathcal{H}_{1} \oplus \mathcal{H}_{2}\right)^{+} \star \mathcal{L} \simeq\left(\mathcal{H}_{1} \star \mathcal{L}\right) \oplus\left(\mathcal{H}_{2} \star \mathcal{L}\right)$ 
2. $\mathcal{H} \star\{0\} \simeq \mathcal{H}^{+}$

3. $\{0\} \star \mathcal{L} \simeq \mathcal{L}^{+\otimes \mathbb{N}}$

Our next goal is to define the product of maps between Hilbert spaces.

Definition 3.2.4. Let $\mathcal{H}_{1}, \mathcal{H}_{2}, \mathcal{L}_{1}, \mathcal{L}_{2}$ be Hilbert spaces, $\mathcal{K}_{1}=\mathcal{H}_{1}^{+} \oplus \mathcal{L}_{1}$ and $\mathcal{K}_{2}=\mathcal{H}_{2}^{+} \oplus$ $\mathcal{L}_{2}, S: \mathcal{H}_{1}^{+} \rightarrow \mathcal{H}_{2}^{+}$and $T: \mathcal{K}_{1} \rightarrow \mathcal{K}_{2}$ bounded maps, and $V: \mathcal{L}_{1} \rightarrow \mathcal{L}_{2}$ a contraction. Define the bounded maps $S \star V: \mathcal{H}_{1} \star \mathcal{L}_{1} \rightarrow \mathcal{H}_{2} \star \mathcal{L}_{2}$ and $T \widehat{\star} V: \mathcal{H}_{1} \star \mathcal{L}_{1} \rightarrow \mathcal{H}_{2} \star \mathcal{L}_{2}$ as follows: Let $V^{+}=V \oplus \operatorname{id}_{\mathbb{C}}: \mathcal{L}_{1}^{+} \rightarrow \mathcal{L}_{2}^{+}$and let $V^{+\otimes \mathbb{N}}: \mathcal{L}_{1}^{+\otimes \mathbb{N}} \rightarrow \mathcal{L}_{2}^{+\otimes \mathbb{N}}$ be the limit of the contractions $V^{+\otimes n}: \mathcal{L}_{1}^{+\otimes n} \rightarrow \mathcal{L}_{2}^{+\otimes n}$. Then $S \star V: \mathcal{H}_{1} \star \mathcal{L}_{1} \rightarrow \mathcal{H}_{2} \star \mathcal{L}_{2}$ is the operator $S \otimes V^{+\otimes \mathbb{N}}: \mathcal{H}_{1}^{+} \otimes \mathcal{L}_{1}^{+\otimes \mathbb{N}} \rightarrow \mathcal{H}_{2}^{+} \otimes \mathcal{L}_{2}^{+\otimes \mathbb{N}}$ composed with the unitary equivalences of Proposition 3.2.2 similarly, $T \widehat{\star} V$ is the operator $T \otimes V^{+\otimes \mathbb{N}}: \mathcal{K}_{1} \otimes \mathcal{L}_{1}^{+\otimes \mathbb{N}} \rightarrow \mathcal{K}_{2} \otimes \mathcal{L}_{2}^{+\otimes \mathbb{N}}$ composed with the appropriate unitary equivalences.

By following the sequence of maps in the proof of Proposition 3.2 .2 , we can calculate how product maps act on the various summands of $\mathcal{H}_{1} \star \mathcal{L}_{1}$.

Proposition 3.2.5. Let $\mathcal{H}_{1}, \mathcal{H}_{2}, \mathcal{L}_{1}, \mathcal{L}_{2}$ be Hilbert spaces, and for $i=1,2$ let $\mathcal{K}_{i}=$ $\mathcal{H}_{i}^{+} \oplus \mathcal{L}_{i} . \quad$ Let $\mathcal{H}_{1}^{+} \stackrel{S}{\rightarrow} \mathcal{H}_{2}^{+}$and $\mathcal{K}_{1} \stackrel{T}{\rightarrow} \mathcal{K}_{2}$ be bounded operators and $\mathcal{L}_{1} \stackrel{V}{\rightarrow} \mathcal{L}_{2}$ a contraction. For each $n \geq 0$ let $V^{(n)}$ denote $V^{+\otimes n} \otimes V: \mathcal{L}_{1}^{+\otimes n} \otimes \mathcal{L}_{1} \rightarrow \mathcal{L}_{2}^{+\otimes n} \otimes \mathcal{L}_{2}$.

Let $h \in \mathcal{H}_{1}^{+}, h_{0} \in \mathcal{H}_{1}, k \in \mathcal{K}_{1}, \ell \in \mathcal{L}_{1}^{+}$, and $\xi \in \mathcal{L}_{1}^{+\otimes n} \otimes \mathcal{L}_{1}$ for some $n \geq 0$, 
and suppose that

$$
\begin{array}{lrl}
S \Omega_{1}=\alpha \Omega_{2}+y, & & \alpha \in \mathbb{C}, y \in \mathcal{H}_{2} \\
S h_{0}=\beta \Omega_{2}+z, & & \beta \in \mathbb{C}, z \in \mathcal{H}_{2} \\
T h_{0}=\eta+w, & \eta \in \mathcal{H}_{2}, w \in \mathcal{L}_{2}^{+} \\
T \ell=\zeta+u, & \zeta \in \mathcal{H}_{2}, u \in \mathcal{L}_{2}^{+} .
\end{array}
$$

Then

$$
\begin{aligned}
(S \star V) h & =S h \\
(S \star V) \xi & =\alpha V^{(n)} \xi+\left(y \otimes V^{(n)} \xi\right) \\
(S \star V)\left(h_{0} \otimes \xi\right) & =\beta V^{(n)} \xi+\left(z \otimes V^{(n)} \xi\right) \\
(T \widehat{\star} V) k & =T k \\
(T \widehat{\star} V)\left(h_{0} \otimes \xi\right) & =\left(\eta \otimes V^{(n)} \xi\right)+\left(w \otimes V^{(n)} \xi\right) \\
(T \widehat{\star} V)(\ell \otimes \xi) & =\left(\zeta \otimes V^{(n)} \xi\right)+\left(u \otimes V^{(n)} \xi\right) .
\end{aligned}
$$

Next, we develop some of the essential properties of this construction.

Proposition 3.2.6. Let $\mathcal{H}_{1}^{+} \stackrel{S}{\rightarrow} \mathcal{H}_{2}^{+} \stackrel{S^{\prime}}{\rightarrow} \mathcal{H}_{3}^{+}$and $\mathcal{K}_{1} \stackrel{T}{\rightarrow} \mathcal{K}_{2} \stackrel{T^{\prime}}{\rightarrow} \mathcal{K}_{3}$ be bounded maps, and $\mathcal{L}_{1} \stackrel{V}{\rightarrow} \mathcal{L}_{2} \stackrel{V^{\prime}}{\rightarrow} \mathcal{L}_{3}$ contractions.

1. $\left(S^{\prime} \star V^{\prime}\right) \circ(S \star V)=\left(S^{\prime} \circ S\right) \star\left(V^{\prime} \circ V\right)$ and $\left(T^{\prime} \widehat{\star} V^{\prime}\right) \circ(T \widehat{\star} V)=\left(T^{\prime} \circ T\right) \widehat{\star}\left(V^{\prime} \circ V\right)$.

2. If $S$ is the identity map on $\mathcal{H}_{1}=\mathcal{H}_{2}$, and $V$ the identity map on $\mathcal{L}_{1}=\mathcal{L}_{2}$, then $S \star V$ is the identity map on $\mathcal{H}_{1} \star \mathcal{L}_{1}=\mathcal{H}_{2} \star \mathcal{L}_{2}$. Similarly, if $T$ and $V$ are the appropriate identity maps, then so is $T \widehat{\star} V$. 
3. $\|S \star V\| \leq\|S\|\|V\|$ and $\|T \widehat{\star} V\| \leq\|T\|\|V\|$.

4. If $S$ and $V$ are isometries (resp. unitaries), so is $S \star V$; if $T$ and $V$ are isometries (resp. unitaries), so is $T \widehat{\star} V$.

5. $(S \star V)^{*}=S^{*} \star V^{*}$ and $(T \widehat{\star} V)^{*}=T^{*} V^{*}$.

6. If $S$ decomposes as a direct sum $S_{L} \oplus S_{R}: \mathcal{H}_{1} \oplus \mathbb{C} \rightarrow \mathcal{H}_{2} \oplus \mathbb{C}$, then $S \star V$ maps the summands of $\mathcal{H}_{1} \star \mathcal{L}_{1}$ into the corresponding summands of $\mathcal{H}_{2} \star \mathcal{L}_{2}$. That is, if $P_{1}$ is the projection from $\mathcal{H}_{1} \star \mathcal{L}_{1}$ onto any of $\mathcal{H}_{1}^{+}, \mathcal{L}_{1}^{+\otimes n} \otimes \mathcal{L}$, or $\mathcal{H}_{1} \otimes \mathcal{L}_{1}^{+\otimes n} \otimes \mathcal{L}$, and $P_{2}$ the projection from $\mathcal{H}_{2} \star \mathcal{L}_{2}$ onto its corresponding subspace, then

$$
(S \star V) P_{1}=P_{2}(S \star V) .
$$

Similarly, if $T$ decomposes as a direct sum $T_{L} \oplus T_{R}: \mathcal{H}_{1} \oplus \mathcal{L}_{1} \rightarrow \mathcal{H}_{2} \oplus \mathcal{L}_{2}$ and $P_{1}, P_{2}$ are as before, then

$$
(T \widehat{\star} V) P_{1}=P_{2}(T \widehat{\star} V) .
$$

7. If $S$ decomposes as the direct sum $S_{L} \oplus i d_{\mathbb{C}}: \mathcal{H}_{1} \oplus \mathbb{C} \rightarrow \mathcal{H}_{2} \oplus \mathbb{C}$ and $T=S \oplus V$, then $S \star V=T \widehat{\star} V$.

Proof. The first five assertions are simple consequences of the corresponding properties of tensor products of operators. The sixth follows as a special case of Proposition 3.2.5 with $y=\beta=0$ or $w=\zeta=0$, and the seventh is also an easy corollary of Proposition 3.2.5.

Remark 3.2.7. The first two properties say that $-\star-$ and $-\widehat{\star}-$ are bifunctors from (Hilbert spaces, bounded maps $) \times($ Hilbert spaces, contractions $)$ to (Hilbert spaces, 
bounded maps), and the third and fourth imply that they restrict to bifunctors from (Hilbert spaces, contractions) $^{2}$ to (Hilbert spaces, contractions), from (Hilbert spaces, isometries) $^{2}$ to (Hilbert spaces, isometries), and from (Hilbert spaces, unitaries) ${ }^{2}$ to (Hilbert spaces, unitaries).

Remark 3.2.8. Together, the fourth and seventh parts of Proposition 3.2.6 imply that, given isometries $W: \mathcal{H}_{1} \rightarrow \mathcal{H}_{2}$ and $V: \mathcal{L}_{1} \rightarrow \mathcal{L}_{2}$, one obtains an isometry $\mathcal{H}_{1} \star \mathcal{L}_{1} \rightarrow \mathcal{H}_{2} \star \mathcal{L}_{2}$ which may be constructed either as $\left(W \oplus \operatorname{id}_{\mathbb{C}}\right) \star V$ or as $\left(W \oplus \mathrm{id}_{\mathbb{C}} \oplus\right.$ $V) \widehat{\star} V$. The sixth part then implies that this induced isometry maps each summand of $\mathcal{H}_{1} \star \mathcal{L}_{1}$ into the corresponding summand of $\mathcal{H}_{2} \star \mathcal{L}_{2}$.

An important special case of Proposition 3.2.5 occurs when $\mathcal{H}_{1}=\mathcal{H}_{2}, \mathcal{L}_{1}=\mathcal{L}_{2}$, and $V$ is the identity map. In this case we obtain unital representations of both $B\left(\mathcal{H}^{+}\right)$ and $B(\mathcal{K})$ on $\mathcal{H} \star \mathcal{L}$, given by $S \mapsto S \star I$ and $T \mapsto T \widehat{\star} I$.

Proposition 3.2.9. Let $\mathcal{H}$ and $\mathcal{L}$ be Hilbert spaces and $\mathcal{K}=\mathcal{H}^{+} \oplus \mathcal{L}$. Let $\Phi$ : $B\left(\mathcal{H}^{+}\right) \rightarrow B(\mathcal{H} \star \mathcal{L})$ and $\Psi: B(\mathcal{K}) \rightarrow B(\mathcal{H} \star \mathcal{L})$ be the representations induced by the unitary equivalences of Proposition 3.2.2. Let $b \in B\left(\mathcal{H}^{+}\right), a \in B(\mathcal{K}), h \in \mathcal{H}^{+}$, $h_{0} \in \mathcal{H}, k \in \mathcal{K}, \ell \in \mathcal{L}^{+}$, and $\xi \in \mathcal{L}^{+\otimes n} \otimes \mathcal{L}$ for some $n \geq 0$, and suppose that

$$
\begin{array}{rlrl}
b \Omega=\alpha \Omega+y, & & \alpha \in \mathbb{C}, y \in \mathcal{H} \\
b h_{0}=\beta \Omega+z, & & \beta \in \mathbb{C}, z \in \mathcal{H} \\
a h_{0}=\eta+w, & \eta \in \mathcal{H}, w \in \mathcal{L}^{+} \\
a \ell=\zeta+u, & \zeta \in \mathcal{H}, u \in \mathcal{L}^{+} .
\end{array}
$$


Then

$$
\begin{gathered}
\Phi(b) h=h \\
\Phi(b) \xi=\alpha \xi+(y \otimes \xi) \\
\Phi(b)\left(h_{0} \otimes \xi\right)=\beta \xi+(z \otimes \xi) \\
\Psi(a) k=a k \\
\Psi(a)\left(h_{0} \otimes \xi\right)=(\eta \otimes \xi)+(w \otimes \xi) \\
\Psi(a)(\eta \otimes \xi)=(\zeta \otimes \xi)+(u \otimes \xi) .
\end{gathered}
$$

The following is an immediate consequence:

Corollary 3.2.10. In the situation of Proposition 3.2.9, the subspaces $\mathcal{H}^{+}$and $\left(\mathcal{L}^{+\otimes n} \otimes \mathcal{L}\right) \oplus\left(\mathcal{H} \otimes \mathcal{L}^{+\otimes n} \otimes \mathcal{L}\right)$ of $\mathcal{H} \star \mathcal{L}$ are $\Phi$-invariant, while the subspaces $\mathcal{H}^{+} \oplus \mathcal{L}$ and $\left(\mathcal{H} \otimes \mathcal{L}^{+\otimes n} \otimes \mathcal{L}\right) \oplus\left(\mathcal{L}^{+\otimes(n+1)} \otimes \mathcal{L}\right)$ are $\Psi$-invariant.

We visualize this corollary using a stairstep diagram:

$$
\begin{aligned}
\mathcal{H}^{+} & \\
\mathcal{L} \quad(\mathcal{H} \otimes \mathcal{L}) & \\
& \left(\mathcal{L}^{+} \otimes \mathcal{L}\right) \quad\left(\mathcal{H} \otimes \mathcal{L}^{+} \otimes \mathcal{L}\right) \\
& \left(\mathcal{L}^{+\otimes 2} \otimes \mathcal{L}\right) \quad\left(\mathcal{H} \otimes \mathcal{L}^{+\otimes 2} \otimes \mathcal{L}\right)
\end{aligned}
$$

The rows here are $\Phi$-invariant, while the columns are $\Psi$-invariant. Equivalently, $\Phi$ 
and $\Psi$ have staggered block-diagonal decompositions:

$$
\Phi=\left[\begin{array}{cccccc}
* & 0 & 0 & 0 & 0 & \ldots \\
0 & * & * & 0 & 0 & \ldots \\
0 & * & * & 0 & 0 & \ldots \\
0 & 0 & 0 & * & * & \ldots \\
0 & 0 & 0 & * & * & \ldots \\
\vdots & \vdots & \vdots & \vdots & \vdots & \ddots
\end{array}\right], \quad \Psi=\left[\begin{array}{ccccccc}
* & * & 0 & 0 & 0 & 0 & \ldots \\
* & * & 0 & 0 & 0 & 0 & \ldots \\
0 & 0 & * & * & 0 & 0 & \ldots \\
0 & 0 & * & * & 0 & 0 & \ldots \\
0 & 0 & 0 & 0 & * & * & \ldots \\
0 & 0 & 0 & 0 & * & * & \ldots \\
\vdots & \vdots & \vdots & \vdots & \vdots & \vdots & \ddots
\end{array}\right]
$$

We see that a sufficiently long word $\Phi\left(b_{0}\right) \Psi\left(a_{1}\right) \Phi\left(b_{1}\right) \Psi\left(a_{2}\right) \cdots$ applied to a vector in one of these subspaces could have a nonzero component in any other subspace. Keeping track of such components will become important later on.

Remark 3.2.11. For simplicity of definition, we have thus far begun with Hilbert spaces $\mathcal{H}$ and $\mathcal{L}$, and defined the space $\mathcal{K}=\mathcal{H}^{+} \oplus \mathcal{L}$ in terms of them. In application, however, we will begin with an inclusion $H \subset K$ (obtained from Stinespring dilation), select a unit vector $\Omega \in H$, and form the Sauvageot product $H^{-} \star(K \ominus H)$. As noted above, $B\left(H^{-} \star(K \ominus H)\right)$ contains unital copies of both $B(H)$ and $B(K)$. In this alone, however, it is no different from $B(H \otimes K)$ or $B(H * K)$, where $H * K$ denotes the free product of Hilbert spaces in the sense of [Voi85. The crucial difference is that, when both are represented on $H^{-} \star(K \ominus H)$, the copy of $B(H)$ is a corner of the copy of $B(K)$; if $H \subset K$ is a Stinespring dilation, the compression will implement a given unital completely positive map. 


\subsection{Sauvageot Products of $\mathrm{C}^{*}$-Algebras and $\mathrm{W}^{*}$-Algebras}

We now begin our construction of the Sauvageot product of unital $\mathrm{C}^{*}$-algebras with respect to a unital completely positive map; the construction requires the choice of a state on one of the $\mathrm{C}^{*}$-algebras, prompting the following definition.

Definition 3.3.1. A CPC $^{*}$-tuple (resp. CPW $^{*}$-tuple) is a quadruple $(A, B, \phi, \omega)$ where $A$ and $B$ are unital $\mathrm{C}^{*}$-algebras (resp. $\mathrm{W}^{*}$-algebras), $\phi: A \rightarrow B$ a unital (normal) completely positive map, and $\omega$ a (normal) state on $B$. The term CP-tuple will refer to $\mathrm{CPC}^{*}$ - and $\mathrm{CPW}^{*}$-tuples together. A CP-tuple is said to be faithful if $\omega$ is a faithful state.

Definition 3.3.2. A representation of a $\operatorname{CPC}^{*}$-tuple $(A, B, \phi, \omega)$ is a sextuple $\left(H, \Omega, \pi_{R}, K, V, \pi_{L}\right)$ where

1. $H$ is a Hilbert space

2. $\Omega \in H$ is a unit vector

3. $\pi_{R}: B \rightarrow B(H)$ is a unital *-homomorphism such that $\left\langle\Omega, \pi_{R}(\cdot) \Omega\right\rangle=\omega(\cdot)$

4. $K$ is a Hilbert space

5. $V: H \rightarrow K$ is an isometry

6. $\pi_{L}: A \rightarrow B(K)$ is a unital *-homomorphism such that $V^{*} \pi_{L}(\cdot) V=\pi_{R}(\phi(\cdot))$.

For a $\mathrm{CPW}^{*}$-tuple, we also require that $\pi_{R}$ and $\pi_{L}$ be normal. A representation is right-faithful if $\pi_{R}$ is injective (which is automatically the case for a representation 
of a faithful CP-tuple), and left-faithful if $\pi_{L}$ is injective. We also refer to $\left(H, \Omega, \pi_{R}\right)$ satisfying the first three criteria as a representation of $(A, \omega)$.

From now until Definition 3.3.9 we fix a CP-tuple $(A, B, \phi, \omega)$ and a rightfaithful representation $\left(H, \Omega, \pi_{R}, K, V, \pi_{L}\right)$. We introduce the following additional notation:

- $L=K \ominus V H$

- $\mathfrak{H}=H^{-} \star L$

- $\psi_{L}: A \rightarrow B(\mathfrak{H})$ and $\psi_{R}: B \rightarrow B(\mathfrak{H})$ are the compositions of $\pi_{L}$ and $\pi_{R}$ with the representations of Proposition 3.2.9

- $A \star B$ is the $\mathrm{C}^{*}$-subalgebra (or von Neumann subalgebra, in case of a $\mathrm{CPW}^{*}$ tuple) of $B(\mathfrak{H})$ generated by the images of $\psi_{L}$ and $\psi_{R}$

- $H^{\prime}=H \ominus \overline{\pi_{R}(B) \Omega}$, which could be zero

- $q_{n}$ for $n \geq 0$ is the projection from $\mathfrak{H}$ onto the subspace $H^{\prime} \otimes L^{+\otimes n} \otimes L$ of $H \otimes L^{+\otimes n} \otimes L$

- $\mathfrak{C}: B(\mathfrak{H}) \rightarrow B(\mathfrak{H})$ is the non-unital conditional expectation

$$
\mathfrak{C}(T)=P_{H} T P_{H}+\sum_{n=0}^{\infty} q_{n} T q_{n}
$$

- $\varpi$ is the vector state on $B(\mathfrak{H})$ corresponding to $\Omega$.

\section{Proposition 3.3.3.}

$$
\mathfrak{C} \circ \psi_{L}=\mathfrak{C} \circ \psi_{R} \circ \phi
$$


Proof. For $a \in A$ and $h \in H$, let $\pi_{L}(a) h=x+\ell$ with $x \in h$ and $\ell \in L$; then $x=P_{H} \pi_{L}(a) P_{H} h=\pi_{R}(\phi(a)) h$. It follows from Proposition 3.2.9 that $\psi_{L}(a) h=x+\ell$, so that

$$
P_{H} \psi_{L}(a) P_{H}=x=P_{H} \pi_{R}(\phi(a)) P_{H}
$$

Similarly, $q_{n} \psi_{L}(a) q_{n}=q_{n} \pi_{R}(\phi(a)) q_{n}$ for all $n \geq 0$. Summing over $n$ yields the result.

For the next lemma and proposition we use $E_{n}$ to denote the subspace $L^{+\otimes n} \otimes L$ of $\mathfrak{H}$.

Lemma 3.3.4. Let $\zeta \in E_{n}$.

1. Let $a \in A$ and $b \in B$ with $\omega(b)=0$. Then

$$
\left[\psi_{L}(a)-\psi_{R}(\phi(a))\right] \psi_{R}(b) \zeta=\left[P_{L^{+}} \pi_{L}(a) \pi_{R}(b) \Omega\right] \otimes \zeta-\omega(\phi(a) b) \zeta \in E_{n} \oplus E_{n+1}
$$

2. More generally, given $a_{1}, \ldots, a_{k} \in A$ and $b_{1}, \ldots, b_{k} \in B$ such that $\omega\left(b_{i}\right)=0$ for $i=1, \ldots, k$, if we define

$$
\zeta_{k}=\left(\prod_{i=1}^{k}\left[\psi_{L}\left(a_{i}\right)-\psi_{R}\left(\phi\left(a_{i}\right)\right)\right] \psi_{R}\left(b_{i}\right)\right) \zeta
$$

then

$$
\zeta_{k} \in \bigoplus_{i=0}^{k} E_{n+i} \text { with } P_{E_{n}}\left(\zeta_{k}\right)=(-1)^{k} \prod_{i=1}^{k} \omega\left(\phi\left(a_{i}\right) b_{i}\right) \zeta
$$

Proof. The stipulation that $\omega(b)=0$ implies that $\pi_{R}(b) \Omega \in H^{-}$, so that

$$
\xi:=\psi_{R}(b) \zeta=\left(\pi_{R}(b) \Omega\right) \otimes \zeta \in H^{-} \otimes E_{n}
$$


where we have used the calculations in Proposition (3.2.9). We have now to apply two different operators to $\xi$ and subtract the results. First, when we apply $\psi_{R}(\phi(a))$ we get

$$
\psi_{R}(\phi(a)) \xi=\left(\pi_{R}(\phi(a) b) \Omega\right) \otimes \zeta=[\omega(\phi(a) b) \Omega] \otimes \eta+\left[P_{H^{-}} \pi_{R}(\phi(a) b) \Omega\right] \otimes \zeta
$$

by virtue of the fact that $\pi_{R}(\beta) \Omega=\omega(\beta) \Omega+P_{H^{-}}\left(\pi_{R}(\beta) \Omega\right)$ for all $b \in B$. Secondly, we apply $\psi_{L}(a)$ as follows:

$$
\begin{aligned}
\psi_{L}(a) \xi & =\left(\pi_{L}(a) \pi_{R}(b) \Omega\right) \otimes \zeta \\
& =\left[\left(P_{H^{-}} \pi_{L}(a) \pi_{R}(b) \Omega\right) \oplus\left(P_{L^{+}} \pi_{L}(a) \pi_{R}(b) \Omega\right)\right] \otimes \zeta \\
& =\left[\left(P_{H^{-}} P_{H^{\prime}} \pi_{L}(a) \pi_{R}(b) \Omega\right) \oplus\left(P_{L^{+}} \pi_{L}(a) \pi_{R}(b) \Omega\right)\right] \otimes \zeta \\
& =\left[\left(P_{H^{-}} \pi_{R}(\phi(a) b) \Omega\right) \otimes \zeta\right] \oplus\left[\left(P_{L^{+}} \pi_{L}(a) \pi_{R}(b) \Omega\right) \otimes \zeta\right] .
\end{aligned}
$$

Subtracting yields the desired result. The second assertion of the lemma follows by induction.

We now connect the current material to chapter 2 .

Proposition 3.3.5. $\left(B(\mathfrak{H}), \psi_{L}, \psi_{R}, \mathfrak{C}, \varpi\right)$ is a right-liberating representation of $(A, B, \phi)$.

Proof. The first two properties of a right-liberating representation are easy to verify:

1. This was Proposition 3.3.3.

2. Since $H$ and each $H^{\prime} \otimes E_{n}$ are $\psi_{R}$-invariant subspaces by Proposition 3.2.9, their projections all commute with $\psi_{R}$, so that $\mathfrak{C}$ is a $\psi_{R}(B)$-bimodule map. 
For the last, let $\xi \in H$ and let $a_{1}, \ldots, a_{n} \in A, b_{1}, \ldots, b_{n} \in B$ such that $\omega\left(b_{2}\right)=\cdots=$ $\omega\left(b_{n}\right)=0$. Define the operators

$$
T_{k}=\left(\psi_{L}\left(a_{k}\right)-\psi_{R}\left(\phi\left(a_{k}\right)\right)\right) \psi_{R}\left(b_{k}\right)
$$

on $\mathfrak{H}$, and the vectors

$$
\zeta_{k}=T_{k} \ldots T_{1} \xi \in \mathfrak{H}
$$

We will show by induction that $\zeta_{k} \in \bigoplus_{j=0}^{k-1} E_{j}$, which is contained in the kernel of $P_{H}$; it will follow that $P_{H} T_{k} \ldots T_{1} P_{H}=0$. For the base case $k=1$, we have $\psi_{R}\left(b_{1}\right) \xi=$ $\pi_{R}\left(b_{1}\right) \xi$, so that $\psi_{R}\left(\phi\left(a_{1}\right)\right) \psi_{R}\left(b_{1}\right) \xi=\pi_{R}\left(\phi\left(a_{1}\right) b_{1}\right) \xi$. We also have

$$
\begin{aligned}
\psi_{L}\left(a_{1}\right) \psi_{R}\left(b_{1}\right) \xi & =\pi_{L}\left(a_{1}\right) \pi_{R}\left(b_{1}\right) \xi \\
& =\left(P_{L} \pi_{L}\left(a_{1}\right) \pi_{R}\left(b_{1}\right) \xi \oplus P_{H} \pi_{L}\left(a_{1}\right) \pi_{R}\left(b_{1}\right) \xi\right) \\
& =\left(P_{L} \pi_{L}\left(a_{1}\right) \pi_{R}\left(b_{1}\right) \xi \oplus P_{H} \pi_{L}\left(a_{1}\right) P_{H} \pi_{R}\left(b_{1}\right) \xi\right) \\
& =\left(P_{L} \pi_{L}\left(a_{1}\right) \pi_{R}\left(b_{1}\right) \xi \oplus \pi_{R}\left(\phi\left(a_{1}\right)\right) \pi_{R}\left(b_{1}\right) \xi\right)
\end{aligned}
$$

and subtracting yields

$$
\zeta_{1}=P_{L} \pi_{L}\left(a_{1}\right) \pi_{R}\left(b_{1}\right) \xi \in E_{0}
$$

as desired. The inductive step is immediate from Lemma 3.3.4.

Similarly, for $\xi \in H^{\prime}$ and $\eta \in E_{n}, \psi_{R}\left(b_{1}\right)(\xi \otimes \eta)=\left(\pi_{R}\left(b_{1}\right) \xi\right) \otimes \eta$ so that

$$
\psi_{R}\left(\phi\left(a_{1}\right)\right) \psi_{R}\left(b_{1}\right)(\xi \otimes \eta)=\left[\pi_{R}\left(\phi\left(a_{1}\right) b_{1}\right) \xi\right] \otimes \eta
$$


Then

$$
\begin{aligned}
\psi_{L}\left(a_{1}\right) \psi_{R}\left(b_{1}\right)(\xi \otimes \eta) & =\psi_{L}\left(a_{1}\right)\left[\left(\pi_{R}\left(b_{1}\right) \xi\right) \otimes \eta\right] \\
& =\left[\pi_{L}\left(a_{1}\right) \pi_{R}\left(b_{1}\right) \xi\right] \otimes \eta \\
& =\left[P_{L} \pi_{L}\left(a_{1}\right) \pi_{R}\left(b_{1}\right) \xi \oplus P_{H} \pi_{L}\left(a_{1}\right) \pi_{R}\left(b_{1}\right) \xi\right] \otimes \eta \\
& =\left[P_{L} \pi_{L}\left(a_{1}\right) \pi_{R}\left(b_{1}\right) \xi \oplus P_{H} \pi_{L}\left(a_{1}\right) P_{H} \pi_{R}\left(b_{1}\right) \xi\right] \otimes \eta \\
& =\left[P_{L} \pi_{L}\left(a_{1}\right) \pi_{R}\left(b_{1}\right) \xi \oplus \pi_{R}\left(\phi\left(a_{1}\right)\right) \pi_{R}\left(b_{1}\right) \xi\right] \otimes \eta
\end{aligned}
$$

and subtracting yields

$$
\zeta_{1}=\left[P_{L} \pi_{L}\left(a_{1}\right) \pi_{R}\left(b_{1}\right) \xi\right] \otimes \eta \in E_{n+1}
$$

It follows by induction that $\zeta_{k} \in \bigoplus_{j=0}^{k-1} E_{j+n}$, so that $q_{n} \zeta_{k}=0$; hence $q_{n} T_{k} \ldots T_{1} q_{n}=0$. Summing over $n$, we have $\mathfrak{C}\left(T_{k} \ldots T_{1}\right)=0$.

\section{Corollary 3.3.6.}

$$
\mathfrak{C}(A \star B)=\mathfrak{C}\left(\psi_{R}(B)\right)
$$

Proof. This is an immediate consequence of Proposition 3.3.5 together with Corollary 2.4 .5 and the norm continuity and normality of $\mathfrak{C}$.

Before making our next definition, we note that the right-faithfulness of our representation guarantees that $b \mapsto P_{H} \psi_{R}(b) P_{H}$ is injective, so that $\mathfrak{C} \circ \psi_{R}$ is injective as well.

Definition 3.3.7. The Sauvageot retraction for the given tuple and representation is the map $\theta: A \star B \rightarrow B$ given by

$$
\theta=\left(\mathfrak{C} \circ \psi_{R}\right)^{-1} \circ \mathfrak{C}
$$


The Sauvageot retraction is well-defined by Corollary 3.3.6, and is a retraction with respect to $\psi_{R}$; furthermore, as a consequence of Proposition 3.3 .3 , it factors $\phi$ in the sense that

$$
\theta \circ \psi_{L}=\phi
$$

Furthermore, the following is an immediate consequence of Proposition 3.3 .5 .

Corollary 3.3.8. $\left(A \star B, \psi_{L}, \psi_{R}, \psi_{R} \circ \theta, \varpi\right)$ is a right-liberating representation of $(A, B, \phi)$.

Definition 3.3.9. Given a CP-tuple $(A, B, \phi, \omega)$ and a right-liberating representation $\left(H, \Omega, \pi_{R}, K, V, \pi_{L}\right)$, the Sauvageot product of the tuple realized by the representation is the tuple $\left(A \star B, \psi_{L}, \psi_{R}, \theta\right)$ of objects constructed as above.

\subsection{Induced Morphisms and Uniqueness}

We pause now to consider an analogy with other product constructions. In building either the (minimal) tensor product or the free product of $\mathrm{C}^{*}$-algebras $A$ and $B$, one can proceed as follows:

1. Represent $A$ and $B$ on Hilbert spaces $H$ and $K$

2. Form the product Hilbert space $H \otimes K$ or $H * K$

3. Lift the representations of $A$ and $B$ to representations of each on this product Hilbert space

4. Take the $\mathrm{C}^{*}$-subalgebra generated by the images of these representations. 
In both cases, one can show that the resulting $\mathrm{C}^{*}$-algebra $A \otimes B$ or $A * B$ is, up to isomorphism, independent of the choice of the representations of $A$ and $B$ provided both are faithful.

We have followed the same outline in constructing $A \star B$, and come now to the question of the independence of this object from the representations used to produce it. It turns out that we need some more complicated hypotheses on the representation, resulting from the fact that a representation of a $\mathrm{CPC}^{*}$-tuple is more complicated than a representation of a $\mathrm{C}^{*}$-algebra, as well as the fact that the product $A \star B$ comes with the additional information of a retraction onto $B$.

Definition 3.4.1. Let $(A, B, \phi, \omega)$ be a CP-tuple, $\left(H, \Omega, \pi_{R}, K, V, \pi_{L}\right)$ a representation, and $L=K \ominus V H$.

- A decomposition of the representation is a pair $\left(L^{\prime}, L^{\prime \prime}\right)$ of $\pi_{L}$-invariant subspaces $L^{\prime} \subset L$ and $L^{\prime \prime} \subset L^{+}$, with the properties

$$
\begin{gathered}
L \subset L^{\prime}+\overline{\pi_{L}(A) V H} \\
L^{+} \subset L^{\prime \prime}+\overline{\pi_{L}(A) V H^{-}} .
\end{gathered}
$$

- A decomposition is faithful if the subrepresentation $\left.\pi_{L}\right|_{L^{\prime}}$ is faithful.

- A representation for which there exists a faithful decomposition is faithfully decomposable.

- A representation is right-faithful if $\pi_{R}$ is faithful; note that any representation of a faithful $\mathrm{CPC}^{*}$-tuple or $\mathrm{CPW}^{*}$-tuple is automatically right-faithful. 
- A representation is left-faithful if $\pi_{L}$ is faithful; note that every faithfully decomposable representation is left-faithful.

- A representation is faithful if it is right-faithful and faithfully decomposable.

Proposition 3.4.2. Every CP-tuple has a faithful representation.

Proof. We begin by letting $\left(H, \Omega, \pi_{R}\right)$ be the GNS construction for $(B, \omega)$; if $\omega$ is not faithful, we replace $\left(H, \pi_{R}\right)$ by its direct sum with some faithful representation of $B$. This guarantees that our representation will be right-faithful.

Next, let $\left(K, V, \pi_{L}\right)$ be the minimal Stinespring dilation of $\pi_{R} \circ \phi$, and let $L^{\prime}=K \ominus \overline{\pi_{L}(A) V H}$ and $L^{\prime \prime}=K \ominus \overline{\pi_{L}(A) H^{-}}$. If $\left.\pi_{L}\right|_{L^{\prime}}$ is not faithful, we replace $\left(K, \pi_{L}\right)$ by its direct sum with some faithful representation of $A$, thereby guaranteeing faithful decomposability.

When some of these additional hypotheses are satisfied, we can define a retraction from $A \star B$ to $A$ which has properties analogous to the retraction $A \star B \stackrel{\theta}{\rightarrow} B$ already discussed. We continue our standard notation for a CP-tuple, a right-faithful representation, and the corresponding realization of the Sauvageot product, and now fix a decomposition $\left(L^{\prime}, L^{\prime \prime}\right)$ as well (not assumed faithful unless specified). We introduce additional notation:

- $E_{0}^{\prime}$ is the subspace $L^{\prime} \subset L$

- For $n \geq 1, E_{n}^{\prime}$ is the subspace $L^{\prime \prime} \otimes L^{+\otimes(n-1)} \otimes L \subset E_{n}$

- For all $n \geq 0, p_{n}$ is the projection from $\mathfrak{H}$ onto $E_{n}^{\prime}$ 
- For all $n \geq 0, F_{n}=H \otimes E_{n}$ and $F_{n}^{\prime}=H^{\prime} \otimes E_{n}$; recall that $q_{n}$ is the projection onto $F_{n}^{\prime}$

- $E_{-1}=\mathbb{C} \Omega$ and $F_{-1}=H^{-}$.

Definition 3.4.3. The left corner map for the given realization and decomposition is the non-unital conditional expectation $\mathfrak{C}^{\prime \prime}$ on $B(\mathfrak{H})$ defined by

$$
\mathfrak{C}^{\prime}(T)=\sum_{n=0}^{\infty} p_{n} T p_{n}
$$

Lemma 3.4.4. Let $J \subset A \star B$ be an ideal contained in the intersection of the kernels of $\mathfrak{C}$ and $\mathfrak{C}^{\prime}$. Then $J=\{0\}$.

Proof. Let $J^{0}$ denote the annihilator of $J$ in $\mathfrak{H}$, i.e. the largest (necessarily closed) subspace such that $J J^{0}=\{0\}$.

1. For any $\alpha \in J$, note that $\alpha^{*} \alpha \in J$; then

$$
\left(\alpha P_{H}\right)^{*}\left(\alpha P_{H}\right)=P_{H} \alpha^{*} \alpha P_{H} \leq \mathfrak{C}\left[\alpha^{*} \alpha\right]=0
$$

so that $\alpha P_{H}=0$. Similarly, $\alpha q_{n}=\alpha p_{n}=0$ for all $n$. Hence $H \subseteq J^{0}$, and for each $n \geq 0, E_{n}^{\prime} \subseteq J^{0}$ and $H^{\prime} \otimes E_{n} \subseteq J^{0}$.

2. We will prove by induction that $E_{n} \subseteq J^{0}$ and $F_{n} \subseteq J^{0}$; the base case $n=-1$ was just established, since $E_{-1} \oplus F_{-1}=H \subset J^{0}$.

3. Suppose $E_{n} \subseteq J^{0}$ and $F_{n} \subseteq J^{0}$.

(a) Since $J \psi_{L}(A) F_{n} \subseteq J F_{n}=\{0\}$, we have $\psi_{L}(A) F_{n} \subseteq J^{0}$. 
(b) By Proposition (3.2.9), $\left[\psi_{L}(A) H^{-}\right] \otimes E_{n} \subseteq \psi_{L}(A) F_{n}$, so that $\left[\psi_{L}(A) H^{-}\right] \otimes$ $E_{n} \subseteq J^{0}$, for the case $n \geq 0$; for $n=-1$, we have $\psi_{L}(A) H \subseteq J^{0}$.

(c) Since we already know $L^{\prime} \subseteq J^{0}$ (in the case $n=-1$ ) or $L^{\prime \prime} \otimes E_{n} \subseteq J^{0}$ (in the case $n \geq 0$ ), it follows that $E_{n+1}=L \subset \overline{\psi_{L}(A) H}+L^{\prime} \subseteq J^{0}$ for the case $n=-1$, or $E_{n+1}=L^{+} \otimes E_{n} \subset\left[\overline{\pi_{L}(A) H^{-}}+L^{\prime \prime}\right] \otimes E_{n} \subseteq J_{0}$ for the case $n \geq 0$.

(d) Since $J \psi_{R}(B) E_{n+1} \subseteq J E_{n+1}=\{0\}$, we have $\psi_{R}(B) E_{n+1} \subseteq J^{0}$.

(e) By Proposition (3.2.9), this implies $\overline{\pi_{R}(B) \Omega} \otimes E_{n+1} \subseteq \overline{\psi_{R}(B) E_{n+1}} \subseteq J^{0}$.

(f) Since we also have $F_{n}^{\prime} \subseteq J^{0}$,

$$
F_{n+1}=H^{-} \otimes E_{n+1} \subseteq H \otimes E_{n+1}=\left(\overline{\pi_{R}(B) \Omega}+H^{\prime}\right) \otimes E_{n+1} \subseteq \overline{\psi_{R}(B) E_{n+1}}+F_{n+1}^{\prime} \subseteq J^{0}
$$

Remark 3.4.5. We note that if $L^{\prime}$ and $L^{\prime \prime}$ are both zero (for instance, when $K$ is given by a minimal Stinespring dilation), then $\mathfrak{C}^{\prime}$ is the zero map; in this case, the lemma says that $\mathfrak{C}$ has no nontrivial ideals in its kernel. This corresponds to the fact that $A$ and $B$ together move $H$ around to all the other components of $\mathfrak{H}$, in the sense that $\overline{(A \star B) H}=\mathfrak{H}$. On the other extreme, if $\left(L^{\prime}, L^{\prime \prime}\right)$ is a faithful decomposition, one has instead that $\overline{(A \star B) H+(A \star B) L^{\prime}+(A \star B) L^{\prime \prime}}=\mathfrak{H}$, but none of $H, L^{\prime}, L^{\prime \prime}$ by itself is enough to reach all of $\mathfrak{H}$. As a result, $\mathfrak{C}$ and $\mathfrak{C}^{\prime}$ may each contain ideals in their kernel, but these ideals are "orthogonal" in the sense of the lemma.

Proposition 3.4.6. Let $\hat{\omega}: B \rightarrow A$ be the unital completely positive map $\hat{\omega}(b)=$ $\omega(b)$ 1. Then $\mathfrak{C}^{\prime \prime} \circ \psi_{L} \circ \hat{\omega}=\mathfrak{C}^{\prime \prime} \circ \psi_{R}$. 
Proof. Given any $b \in B$, we have $\pi_{R}(b) \Omega=\omega(b) \Omega+h_{0}$ for some $h_{0} \in H^{-}$. By Proposition 3.2.9, it follows that, for any $\xi \in E_{n}^{\prime}, \psi_{R}(b) \xi=\omega(b) \xi+h_{0} \otimes \xi$ and therefore that $p_{n} \psi_{R}(b) \xi=\omega(b) \xi$; hence,

$$
p_{n} \psi_{R}(b) p_{n}=\omega(b) \mathbf{1}_{E_{n}}=p_{n} \omega(b) \mathbf{1} p_{n}=p_{n} \psi_{L}(\hat{\omega}(b)) p_{n}
$$

Summing over $n$ yields the result.

Proposition 3.4.7. $\left(B(\mathfrak{H}), \psi_{L}, \psi_{R}, \mathfrak{C}^{\prime}, \varpi\right)$ is a left-liberating representation of $(A, B, \phi)$.

Proof. The first two properties of a left-liberating proposition are easy to check:

1. This was Proposition 3.4.6.

2. By Proposition 3.2 .9 , all of the $E_{n}^{\prime}$ are $\psi_{L}$-invariant, so their projections commute with $\psi_{L}$; hence $\mathfrak{C}^{\prime}$ is a $\psi_{L}(A)$-bimodule map.

For the last, let $a_{1}, \ldots, a_{n} \in A$ and $b_{0}, \ldots, b_{n} \in B$ with $\omega\left(b_{i}\right)=0$. Let $m \geq 0$ and $\xi \in E_{m}^{\prime}$. By Lemma 3.3.4,

$$
\prod_{k=1}^{n}\left[\psi_{L}\left(a_{k}\right)-\psi_{R}\left(\phi\left(a_{k}\right)\right)\right] \xi \in \bigoplus_{k=0}^{m} E_{n+k}
$$

Since $\omega\left(b_{0}\right)=0$, it follows that $\pi_{R}\left(b_{0}\right) \Omega \in H^{-}$, so that by Proposition 3.2 .9 we obtain

$$
\psi_{R}\left(b_{0}\right) \prod_{k=1}^{n}\left[\psi_{L}\left(a_{k}\right)-\psi_{R}\left(\phi\left(a_{k}\right)\right)\right] \xi \in \bigoplus_{k=0}^{m} H^{-} \otimes E_{n+k}
$$

From this we see that

$$
p_{n} \psi_{R}\left(b_{0}\right) \prod_{k=1}^{n}\left[\psi_{L}\left(a_{k}\right)-\psi_{R}\left(\phi\left(a_{k}\right)\right)\right] p_{n}=0
$$

and summing over $n$ finishes the proof. 
Corollary 3.4.8. $\mathfrak{C}^{\prime}(A \star B)=\mathfrak{C}^{\prime}(A)$.

Proof. This is an immediate consequence of Proposition 3.4.7. Corollary 2.5.2, and the contractivity (and, in case $(A, B, \phi, \omega)$ is a $\mathrm{CPW}^{*}$-tuple, the normality) of $\mathfrak{C}^{\prime}$.

In the case of a faithful decomposition, $\mathfrak{C}^{\prime} \circ \psi_{L}$ is injective, which allows us to make the following definition:

Definition 3.4.9. Given a CP-tuple, a faithful representation, and a choice of faithful decomposition, the left retraction for the given tuple and representation is the map $\theta: A \star B \rightarrow A$ given by

$$
\theta=\left(\mathfrak{C}^{\prime} \circ \psi_{L}\right)^{-1} \circ \mathfrak{C}^{\prime}
$$

This is well-defined by Corollary 3.4.8, and is a retraction with respect to $\psi_{L}$.

We come now to the main result of this section.

Theorem 3.4.10. Let $\left(A_{1}, B_{1}, \phi_{1}, \omega_{1}\right)$ and $\left(A_{2}, B_{2}, \phi_{2}, \omega_{2}\right)$ be $C P C^{*}$-tuples (resp. $C P W^{*}$ tuples), $\left(H_{1}, \Omega_{1}, \pi_{R}^{(1)}, K_{1}, V_{1}, \pi_{L}^{(1)}\right)$ a faithful representation of the former, $\left(H_{2}, \Omega_{2}, \pi_{R}^{(2)}, K_{2}, V_{2}, \pi_{L}^{(2)}\right)$ a right-faithful representation of the latter, and $\left(A_{1} \star B_{1}, \psi_{L}^{(1)}, \psi_{R}^{(1)}, \varpi_{1}, \theta_{1}\right)$ and $\left(A_{2} \star B_{2}, \psi_{L}^{(2)}, \psi_{R}^{(2)}, \varpi_{2}, \theta_{2}\right)$ the Sauvageot products realized by these representations. Let $f: A_{1} \rightarrow A_{2}$ and $g: B_{1} \rightarrow B_{2}$ be unital (normal) *-homomorphisms satisfying $\phi_{2} \circ f=g \circ \phi_{1}$ and $\omega_{2} \circ g=\omega_{1}$. Then there is a unique (normal) unital ${ }^{*}$-homomorphism $f \star g: A_{1} \star B_{1} \rightarrow A_{2} \star B_{2}$ with the properties that

1. $(f \star g) \circ \psi_{L}^{(1)}=\psi_{L}^{(2)} \circ f$

2. $(f \star g) \circ \psi_{R}^{(1)}=\psi_{R}^{(2)} \circ g$ 
3. $\varpi_{2} \circ(f \star g)=\varpi_{1}$

4. $\theta_{2} \circ(f \star g)=g \circ \theta_{1}$

If $f$ and $g$ are both injective and $\left(H_{2}, \Omega_{2}, \pi_{R}^{(2)}, K_{2}, V_{2}, \pi_{L}^{(2)}\right)$ is faithful, then $f \star g$ is injective.

Proof. Let $H=H_{1} \oplus H_{2}, \Omega=\Omega_{1}, \pi_{R}=\pi_{R}^{(1)} \oplus\left(\pi_{R}^{(2)} \circ g\right), K=K_{1} \oplus K_{2}, V=V_{1} \oplus V_{2}$, $\pi_{L}=\pi_{L}^{(1)} \oplus\left(\pi_{L}^{(2)} \circ f\right)$. Then $\left(H, \Omega, \pi_{R}, K, V, \pi_{L}\right)$ is another right-faithful representation of $\left(A_{1}, B_{1}, \phi_{1}, \omega_{1}\right)$. Moreover, if $\left(L_{1}^{\prime}, L_{1}^{\prime \prime}\right)$ is a decomposition for $\left(H_{1}, \ldots, \pi_{L}^{(1)}\right)$, then $L^{\prime}=L_{1}^{\prime} \oplus L_{2}, L^{\prime \prime}=L_{1}^{\prime \prime} \oplus L_{2}$ defines a decomposition $\left(L^{\prime}, L^{\prime \prime}\right)$ of $\left(H, \ldots, \pi_{L}\right)$, and the faithfulness of $\pi_{L}^{(1)}$ on $L_{1}^{\prime}$ implies the faithfulness of $\pi_{L}$ on $L^{\prime}$.

Let $\left(A_{1} \widetilde{\star} B_{1}, \psi_{L}, \psi_{R}, \varpi, \theta\right)$ be the Sauvageot product realized by $\left(H, \ldots, \pi_{L}\right)$, on the Hilbert space $\mathfrak{M}=H^{-} \star L$.

The inclusions of $H_{1}$ into $H$ and of $L_{1}$ into $L$ induce an isometry $W: \tilde{H}_{1} \rightarrow \mathfrak{H}$ as in Remark (3.2.8). Moreover, by Equation (3.1), this isometry satisfies

$$
W \circ \psi_{L}^{(1)}(\cdot)=\psi_{L}(\cdot) \circ W, \quad W \circ \psi_{R}^{(1)}(\cdot)=\psi_{R}(\cdot) \circ W
$$

Let $\Psi$ be the restriction to $A_{1} \widetilde{\star} B_{1}$ of the (normal) unital $\mathrm{CP}$ map $T \mapsto W^{*} T W$, which maps $B(\mathfrak{H})$ to $B\left(\mathfrak{H}_{1}\right)$. It follows from Equation (3.4) that the image of $W$ is invariant under $\psi_{L}$ and $\psi_{R}$, so that $W W^{*}$ commutes with $A_{1} \widetilde{\star} B_{1}$. Then

$$
\Psi(X Y)=W^{*} X Y W=W^{*} W W^{*} X Y W=W^{*} X W W^{*} Y W=\Psi(X) \Psi(Y)
$$

for $X, Y \in A_{1} \widetilde{\star} B_{1}$, so that $\Psi$ is a *-homomorphism.

Next, we show that $\Psi$ intertwines the representations, states, and retractions: 
- $\Psi \circ \psi_{L}=\psi_{L}^{(1)}$

- $\Psi \circ \psi_{R}=\psi_{R}^{(1)}$

- $\varpi_{1} \circ \Psi=\varpi$

- $\theta_{1} \circ \Psi=\theta$

- $\theta_{1}^{\prime} \circ \Psi=\theta^{\prime}$

The first three are immediate consequences of Equation (3.4). For the fourth and fifth, we have by Equation (3.1) that

$\Psi \circ \mathfrak{C}(T)=W^{*} P_{H} T P_{H} W^{*}+\sum_{n} W^{*} p_{n} T p_{n} W=P_{H_{1}} W^{*} T W P_{H_{1}}+\sum_{n} p_{n, 1} W^{*} T W p_{n, 1}=\mathfrak{C}_{1} \circ \Psi(T)$

so that $\Psi \circ \mathfrak{C}=\mathfrak{C}_{1} \circ \Psi$, and similarly for $\mathfrak{C}^{\prime}$ and $\mathfrak{C}_{1}^{\prime}$. Now

$$
\Psi \circ \mathfrak{C} \circ \psi_{R}=\mathfrak{C}_{1} \circ \Psi \circ \psi_{R}=\mathfrak{C}_{1} \circ \psi_{R}^{(1)},
$$

which is invertible; then

$$
\left(\mathfrak{C} \circ \psi_{R}\right)^{-1}=\left(\Psi \circ \mathfrak{C} \circ \psi_{R}\right)^{-1} \circ \Psi \circ \mathfrak{C} \circ \psi_{R} \circ\left(\mathfrak{C} \circ \psi_{R}\right)^{-1}=\left(\Psi \circ \mathfrak{C} \circ \psi_{R}\right)^{-1} \circ \Psi
$$

from which it follows that

$$
\begin{aligned}
\theta & =\left(\mathfrak{C} \circ \psi_{R}\right)^{-1} \circ \mathfrak{C} \\
& =\left(\Psi \circ \mathfrak{C}^{\mathfrak{L}} \circ \psi_{R}\right)^{-1} \circ \Psi \circ \mathfrak{C} \\
& =\left(\mathfrak{C}_{1} \circ \psi_{R}^{(1)}\right)^{-1} \circ \mathfrak{C}_{1} \circ \Psi \\
& =\theta_{1} \circ \Psi
\end{aligned}
$$


and similarly

$$
\theta^{\prime}=\theta_{1}^{\prime} \circ \Psi
$$

Note that $\Psi$ maps into $A_{1} \star B_{1}$, as it maps both $\psi_{L}\left(A_{1}\right)$ and $\psi_{R}\left(B_{1}\right)$ into $A_{1} \star B_{1}$, hence also the $\mathrm{C}^{*}$-algebra (resp. von Neumann algebra) that they generate; moreover, it is onto $A_{1} \star B_{1}$, since its range is a $\mathrm{C}^{*}$-algebra (resp. von Neumann algebra) which includes both $\psi_{L}^{(1)}\left(A_{1}\right)$ and $\psi_{R}^{(1)}\left(B_{1}\right)$.

Next, $\Psi$ is injective, because its kernel is an ideal in $A_{1} \widetilde{\star} B_{1}$ which, by equations 3.5 and 3.6, is contained in the kernels of both $\theta$ and $\theta^{\prime}$, therefore also in the kernels of both $\mathfrak{C}$ and $\mathfrak{C}^{\prime}$, and hence is the zero ideal by Lemma 3.4.4. So $\Psi$ is an isomorphism from $A_{1} \widetilde{\star} B_{1}$ to $A_{1} \star B_{1}$.

We repeat the above analysis for the inclusions of $\mathrm{H}_{2}$ into $\mathrm{H}$ and $\mathrm{K}_{2}$ into $\mathrm{K}$

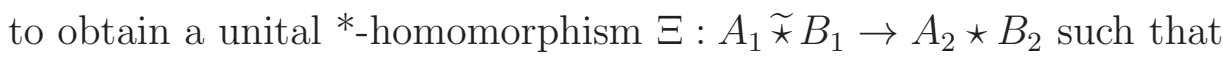

- $\Xi \circ \psi_{L}=\psi_{L}^{(2)} \circ f$

- $\Xi \circ \psi_{R}=\psi_{R}^{(2)} \circ g$

- $\varpi_{2} \circ \Xi=\varpi$

- $\theta_{2} \circ \Xi=g \circ \theta$

We can now define $f \star g=\Xi \circ \Psi^{-1}: A_{1} \star B_{1} \rightarrow A_{2} \star B_{2}$. Then we obtain the enumerated properties of $f \star g$ by combining the lists of properties for $\Psi$ and $\Xi$, as

$$
(f \star g) \circ \psi_{L}^{(1)}=\Xi \circ \Psi^{-1} \circ \psi_{L}^{(1)}=\Xi \circ \psi_{L}=\psi_{L}^{(2)} \circ f
$$

and similarly. 
The uniqueness of $f \star g$ follows from the fact that it is contractive (resp. normal) and is determined on the dense subalgebra of $A_{1} \star B_{1}$ generated by $\psi_{L}^{(1)}\left(A_{1}\right)$ and $\psi_{R}^{(1)}\left(B_{1}\right)$.

Finally, if $f$ and $g$ are both injective and $\left(H_{2}, \ldots, \pi_{L}^{(2)}\right)$ is faithful, we can prove the additional property $\theta_{2}^{\prime} \circ \Xi=\Xi \circ f$, after which we prove $\Xi$ to be injective exactly as we did with $\Psi$ was. Hence $f \star g$ is a composition of injective maps.

Corollary 3.4.11. Let $(A, B, \phi, \omega)$ be a CP-tuple. Then the realizations of the Sauvageot product by any two faithful representations are isomorphic.

For clarity, "isomorphic" here refers to an isomorphism which intertwines the appropriate maps. The proof is simply to take the map $\mathrm{id}_{A} \star \mathrm{id}_{B}$ constructed in the theorem. Based on this corollary, we may now speak of the Sauvageot product of a CP-tuple.

Another special case of interest occurs when one, but not both, of the initial maps is the identity. The results are summarized as follows.

Corollary 3.4.12. Let $A, B$ be unital $C^{*}$-algebras (resp. $W^{*}$-algebras), $A \stackrel{f}{\rightarrow} B$ a unital (normal) *-homomorphism and $C$ another unital $C^{*}$-algebra (resp. $W^{*}$-algebra).

1. Let $B \stackrel{\phi}{\rightarrow} C$ be a (normal) unital $C P$ map and $\omega$ a (normal) state on $C$. Then, for the CP-tuples $(A, C, \phi \circ f, \omega)$ and $(B, C, \phi, \omega)$ with Sauvageot retractions $A \star C \stackrel{\theta}{\rightarrow} C$ and $B \star C \stackrel{\eta}{\rightarrow} C$, the diagrams
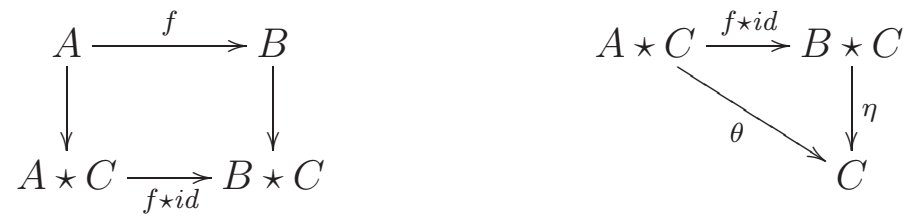
commute.

2. Let $C \stackrel{\phi}{\rightarrow} A$ be a (normal) unital $C P$ map and $\omega$ a (normal) state on $B$. Then, for the $C P$-tuples $(C, A, \phi, \omega \circ f)$ and $(C, B, f \circ \phi, \omega)$, the square

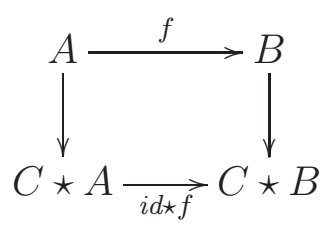

commutes.

The composition of Sauvageot products of maps obeys the obvious functorial property:

Proposition 3.4.13. For $i=1,2,3$ let $\left(A_{i}, B_{i}, \phi_{i}, \omega_{i}\right)$ be CP-tuples, and for $i=1,2$ let $A_{i} \stackrel{f_{i}}{\rightarrow} A_{i+1}$ and $B_{i} \stackrel{g_{i}}{\rightarrow} B_{i+1}$ be (normal) unital *-homomorphisms, such that the diagram

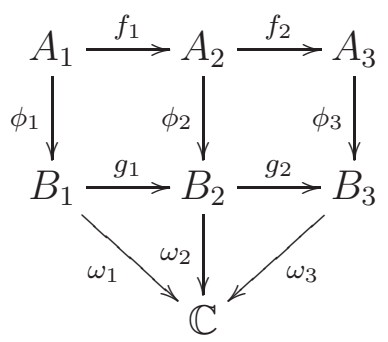

commutes. Then

$$
\left(f_{2} \circ f_{1}\right) \star\left(g_{2} \circ g_{1}\right)=\left(f_{2} \star g_{2}\right) \circ\left(f_{1} \star g_{1}\right) .
$$

Next, we note that the Sauvageot retraction possesses a certain universal property.

Proposition 3.4.14. Let $(A, B, \phi, \omega)$ be a $C P$-tuple, with Sauvageot product $A \star B$ and retraction $\theta: A \star B \rightarrow B$. Suppose $\hat{\theta}: A \star B \rightarrow B$ is another (normal) retraction with 
respect to $\psi_{R}$ such that $\left(A \star B, \psi_{L}, \psi_{R}, \hat{\theta} \circ \psi_{R}, \varpi\right)$ is a right-liberating representation for $(A, B, \phi, \omega)$. Then $\hat{\theta}=\theta$.

Proof. Applying Theorem 2.4.2 to the conditional expectations $\psi_{R} \circ \theta$ and $\psi_{R} \circ \hat{\theta}$, we see that they agree on a dense ${ }^{*}$-subalgebra of $A \star B$, hence on the whole by continuity. Since $\psi_{R}$ is injective, this implies $\theta=\hat{\theta}$.

\subsection{Trivial Cases of the Sauvageot Product}

Tensor products have the property that $A \otimes \mathbb{C} \simeq A \simeq \mathbb{C} \otimes A$ for any commutative unital $\mathrm{C}^{*}$-algebra $A$; similarly, unital free products have the property that $A * \mathbb{C} \simeq A \simeq \mathbb{C} * A$ for any unital $\mathrm{C}^{*}$-algebra $A$. Moreover, amalgamated free products satisfy $A *_{A} A \simeq A$. We now consider analogues of these properties for the Sauvageot product. These are of interest not only for their own sake, but also as the base cases in the inductive system of the next chapter.

Proposition 3.5.1 $(\mathbb{C} \star \mathcal{A} \simeq \mathcal{A})$. Let $\mathcal{A}$ be any unital $C^{*}$-algebra (resp. $W^{*}$-algebra), $v: \mathbb{C} \rightarrow \mathcal{A}$ the embedding of $\mathbb{C}$, and $\omega$ any state (resp. normal state) on $\mathcal{A}$. Then the Sauvageot product $\mathbb{C} \star \mathcal{A}$ of the $C P$-tuple $(\mathbb{C}, \mathcal{A}, v, \omega)$ is isomorphic to $\mathcal{A} ;$ modulo this identification, the embedding $\psi_{L}: \mathbb{C} \rightarrow \mathbb{C} \star \mathcal{A}$ is $v$, and $\psi_{R}: \mathcal{A} \rightarrow \mathbb{C} \star \mathcal{A}$ and $\mathbb{E}: \mathbb{C} \star \mathcal{A} \rightarrow \mathcal{A}$ are both the identity map.

Proof. One can prove this by constructing a representation of this CP-tuple; on the space $\mathfrak{H}$, one has $\psi_{L}$ mapping into $\psi_{R}(\mathcal{A})$, so that the algebra generated by both the images together is isomorphic to $\mathcal{A}$. Alternatively, right-liberation becomes trivial when one of the algebras involved is $\mathbb{C}$, so that $\mathbb{E}$ is multiplicative and hence is a 
*-homomorphic inverse for $\psi_{R}$.

Remark 3.5.2. One might conjecture that, more generally, the Sauvageot product with respect to an embedding is trivial; that is, if $A \stackrel{\iota}{\rightarrow} B$ is an embedding, or equivalently if $A \subset B$ is an embedding, that $A \star B \simeq B$.

This turns out not to be the case. We are interested in whether $\psi_{L}=\psi_{R} \circ \iota$; but on the subspace $L^{\prime}$ in a faithful decomposition, $\psi_{L}$ acts faithfully, whereas $\psi_{R} \circ \iota$ acts in a trivial fashion (in particular, the component in $L^{\prime}$ of $\psi_{R}(\iota(a)) \xi$ for $\xi \in L^{\prime}$ must be a scalar multiple of $\xi)$.

This illustrates an important feature of the Sauvageot product. If we were to start by representing $B$ on some $H$ through the GNS construction, then use Stinespring dilation to obtain a representation of $A$ on $K$, then in the special case that the map from $A$ to $B$ is an embedding (indeed, any homomorphism) one would have $K=H$ and therefore $L=\{0\}$, from which it would follow that $\mathfrak{H} \simeq H$ as well, and $A \star B \simeq B$. But the Sauvageot product is defined with respect to a faithful representation, which involves taking direct sums at various points in the process so as to avoid collapsing into triviality.

Proposition 3.5.3 $(\mathcal{A} \star \mathbb{C} \simeq \mathbb{C})$. Let $\mathcal{A}$ be any unital $C^{*}$-algebra (resp. $W^{*}$-algebra), and $\omega$ any state (resp. normal state) on $\mathcal{A}$. Then the Sauvageot product $\mathcal{A} \star \mathbb{C}$ of the CP-tuple $(\mathcal{A}, \mathbb{C}, \omega, i d \mathbb{C})$ is isomorphic to $\mathcal{A} ;$ modulo this identification, the left embedding $\psi_{L}: \mathcal{A} \rightarrow \mathcal{A} \star \mathbb{C}$ is the identity map, the right embedding $\psi_{R}: \mathbb{C} \rightarrow \mathcal{A} \star \mathbb{C}$ is $v$, and the retraction $\mathbb{E}: \mathcal{A} \star \mathbb{C} \rightarrow \mathbb{C}$ is $\omega$.

Proof. As with the previous proposition. 
Remark 3.5.4. Now given a CP-tuple $(A, B, \phi, \omega)$, one can identify $A$ with $\mathbb{C} \star A$ (resp. $A \star \mathbb{C})$ and $B$ with $\mathbb{C} \star B($ resp. $B \star \mathbb{C})$; it is then natural to ask whether $\phi$ is thereby identified with $\operatorname{id}_{\mathbb{C}} \star \phi\left(\right.$ resp. $\left.\phi \star \mathrm{id}_{\mathbb{C}}\right)$. The answer is yes; indeed, this is a special case of Corollary 3.4.12. 


\section{CHAPTER 4}

\section{ALGEBRAIC C*-DILATIONS THROUGH ITERATED PRODUCTS}

\subsection{Introduction}

Having shown how to construct the Sauvageot product of a CP-tuple, we now broach the question of how to iterate this product in order to construct dilations. For motivation, we return again to the Daniell-Kolmogorov construction as viewed through the lens of the tensor product (Example 1.3.3).

Recall that we begin with a compact Hausdorff space $S$ (the state space of a Markov process), with corresponding path space $\mathscr{S}=S^{[0, \infty)}$; we use $\mathcal{A}$ to denote $C(S)$ and $\mathfrak{A}$ to denote $C(\mathscr{S})$, though we seek here to construct $\mathfrak{A}$ only through $\mathrm{C}^{*}$ algebraic means, without reference to $\mathscr{S}$ except as a guide to understanding. For each finite subset $\gamma \subset[0, \infty)$, we let $\mathcal{A}_{\gamma}$ denote a tensor product of $|\gamma|$ copies of $C(S)$ with itself. When we have constructed $\mathfrak{A}$, we will embed such an $\mathcal{A}_{\gamma}$ into it, representing those functions on the path space which only depend on times in $\gamma$.

For $\beta \leq \gamma$ we can embed $\mathcal{A}_{\beta}$ into $\mathcal{A}_{\gamma}$ by tensoring with $\mathbf{1}$ 's in all the missing coordinates. It is difficult to find notation which makes this more precise while maintaining the basic simplicity of the concept, but here are two attempts. First, an example. If $\gamma=\left\{t_{1}, \ldots, t_{7}\right\}$ with the times listed in increasing order, and $\beta=\left\{t_{2}, t_{5}, t_{6}\right\}$, then one embeds $\mathcal{A}_{\beta}$ into $\mathcal{A}_{\gamma}$ via

$$
f \otimes g \otimes h \longmapsto 1 \otimes f \otimes 1 \otimes 1 \otimes g \otimes h \otimes 1 .
$$

Second, a general observation: Such an embedding can be built from repeated em- 
beddings corresponding to adding a single time, so we may reduce to the case $\beta=$ $\left\{t_{1}, \ldots, t_{n}\right\}$ and $\gamma=\left\{t_{1}, \ldots, t_{k}, \tau, t_{k+1}, \ldots, t_{n}\right\}$ where again we assume the times are in increasing order. In this case the embedding is

$$
f_{1} \otimes \cdots \otimes f_{n} \longmapsto f_{1} \otimes \cdots \otimes f_{k} \otimes 1 \otimes f_{k+1} \otimes \cdots \otimes f_{n}
$$

It is easy to see that the family of embeddings under consideration form an inductive system, so that we may take the limit to obtain a $\mathrm{C}^{*}$-algebra $\mathfrak{A}$ generated by copies of each $\mathcal{A}_{\gamma}$.

We note in passing that the limit construction becomes even simpler when viewed through the lens of the Gelfand functor. Since $\mathcal{A}_{\gamma}$ may be identified with $C\left(S^{\gamma}\right)$, one can consider the inverse system of compact Hausdorff spaces $\left\{S^{\gamma}\right\}$ equipped with the canonical projections. The projective (aka inverse) limit is the path space $\mathscr{S}$, so applying the contravariant equivalence of categories, the inductive (aka direct) limit of the corresponding embeddings is isomorphic to $C(\mathscr{S})$. While elegant, however, this point of view will be of little use in our noncommutative generalizations, since there is no underlying path space to work with.

Having constructed the limit algebra $\mathfrak{A}$, with the embedding $\mathcal{A} \hookrightarrow \mathfrak{A}$ corresponding to the identification of $\mathcal{A}$ with $\mathcal{A}_{\{0\}}$, we are left with the task of constructing the retraction $\mathbb{E}: \mathfrak{A} \rightarrow \mathcal{A}$. We do this by first constructing a consistent family of retractions $\mathcal{A}_{\gamma} \rightarrow \mathcal{A}_{\beta}$ for $\beta \leq \gamma$, then showing how to use a limiting process to induce the retraction $\mathfrak{A} \rightarrow \mathcal{A}$. First, we reduce as before to the case where $\gamma$ contains one 
more point than $\beta$, then retract

$$
f_{1} \otimes \cdots \otimes f_{k} \otimes g \otimes f_{k+1} \otimes \cdots \otimes f_{n} \longmapsto f_{1} \otimes \cdots \otimes\left(f_{k} P_{\tau-t_{k}} g\right) \otimes f_{k+1} \otimes \cdots \otimes f_{n} .
$$

Note that in particular, when $\gamma$ contains 0 and one identifies $\mathcal{A}$ with $\mathcal{A}_{\{0\}}$, repeated application of this rule yields the retraction $\mathcal{A}_{\gamma} \rightarrow \mathcal{A}$ given on simple tensors by

$$
f_{1} \otimes \cdots \otimes f_{n} \longmapsto f_{1} P_{t_{2}-t_{1}}\left(f_{2} P_{t_{3}-t_{2}}\left(f_{3} \cdots P_{t_{n}-t_{n-1}}\left(f_{n}\right)\right) \cdots\right) .
$$

Again, one can check that this family of retractions is consistent with the inductive system, so that it yields a well-defined and contractive map onto $\mathcal{A}$ from the dense subalgebra of $\mathfrak{A}$ generated by the images of all the $\mathcal{A}_{\gamma}$; as this map is contractive, it extends to a retraction on all of $\mathfrak{A}$.

When seeking to carry this method across to the Sauvageot product, one runs into several hurdles. First, one does not form the Sauvageot product merely of two $\mathrm{C}^{*}$-algebras, but rather of a CP-tuple; hence, one cannot begin by defining $\mathcal{A}_{\gamma}=\mathcal{A} \star \cdots \star \mathcal{A}$ without specifying what maps are used between the various copies of $\mathcal{A}$. A related and deeper problem is the failure of associativity; even when the relevant maps have been selected to make the notation well-defined, in general one does not have $(A \star A) \star(A \star A)$ isomorphic to $((A \star A) \star A) \star A$. Hence, we are led to adopt a more laborious inductive construction, though we follow the same high-level strategy as in the commutative case.

For the remainder of the chapter, we fix a unital $\mathrm{C}^{*}$-algebra (resp. $\mathrm{W}^{*}$-algebra) $\mathcal{A}$, a faithful state (resp. faithful normal state) $\omega$ on $\mathcal{A}$, and a $\mathrm{cp}_{0}$-semigroup $\left\{\phi_{t}\right\}$ on $\mathcal{A}$. We use $\mathcal{F}$ to denote the set of finite subsets of $[0, \infty)$. Throughout, we assume 
unless otherwise indicated that times within such sets are listed in increasing order; hence, writing $\gamma=\left\{t_{1}, \ldots, t_{n}\right\}$ implies $t_{1}<\cdots<t_{n}$.

\subsection{Construction of the Inductive System and Limit}

\subsubsection{Objects and Immediate-Tail Morphisms}

Definition 4.2.1. Let $\beta, \gamma \in \mathcal{F}$ with $\gamma=\left\{t_{1}, \ldots, t_{n}\right\}$. We call $\beta$ an initial segment of $\gamma$ if $\beta=\left\{t_{1}, \ldots, t_{m}\right\}$ for some $1 \leq m \leq n$, and a tail of $\gamma$ if $\beta=\left\{t_{\ell}, \ldots t_{n}\right\}$ for some $1 \leq \ell \leq n$. If $\ell=2$ we call $\beta$ an immediate tail with distance $t_{2}-t_{1}$.

We are now able to define the objects of our inductive system, as well as some of the morphisms.

Definition 4.2.2. For nonempty $\gamma \in \mathcal{F}$ we define inductively

1. a unital $\mathrm{C}^{*}$-algebra (resp. $\mathrm{W}^{*}$-algebra) $\mathcal{A}_{\gamma}$

2. a unital embedding $\iota_{\gamma}: \mathcal{A} \rightarrow \mathcal{A}_{\gamma}$

3. a retraction $\epsilon_{\gamma}: \mathcal{A}_{\gamma} \rightarrow \mathcal{A}$

as follows:

- If $\gamma$ is a singleton, then $\mathcal{A}_{\gamma}=\mathcal{A}$ and both $\iota_{\gamma}$ and $\epsilon_{\gamma}$ are the identity.

- If $\beta$ is an immediate tail of $\gamma$ with distance $\tau$, let $\Phi=\phi_{\tau} \circ \epsilon_{\beta}: \mathcal{A}_{\beta} \rightarrow \mathcal{A}$, and form the CP-tuple $\left(\mathcal{A}_{\beta}, \mathcal{A}, \Phi, \omega\right)$. Then $\mathcal{A}_{\gamma}$ is the Sauvageot product $\mathcal{A}_{\beta} \star \mathcal{A}, \iota_{\gamma}$ is the embedding of $\mathcal{A}$ into $\mathcal{A}_{\beta} \star \mathcal{A}$ (denoted $\psi_{R}$ in the previous chapter), and $\epsilon_{\gamma}$ is the Sauvageot retraction from $\mathcal{A}_{\beta} \star \mathcal{A}$ onto $\mathcal{A}$. 
We also define $\mathcal{A}_{\emptyset}=\mathbb{C}$.

Note that this definition also implicitly gives us embeddings $\mathcal{A}_{\beta} \hookrightarrow \mathcal{A}_{\gamma}$ in the special case where $\beta$ is an immediate tail of $\gamma$; this is just the canonical embedding of $\mathcal{A}_{\beta}$ into $\mathcal{A}_{\beta} \star \mathcal{A}$, the map denoted in the previous chapter by $\psi_{L}$.

We turn next to the question of how to embed $\mathcal{A}_{\beta}$ into $\mathcal{A}_{\gamma}$ when $\beta \leq \gamma$ more generally.

\subsubsection{General Morphisms}

Consider now any inclusion $\beta \leq \gamma$ of nonempty elements of $\mathcal{F}$. Let $\gamma=$ $\left\{t_{1}, \ldots, t_{n}\right\}$ and for each $\ell \in\{1, \ldots, n\}$ define subsets $\gamma(\ell) \leq \gamma$ and $\beta(\ell) \leq \beta$ by

$$
\gamma(\ell)=\gamma \cap\left\{t_{\ell}, \ldots, t_{n}\right\}, \quad \beta(\ell)=\beta \cap\left\{t_{\ell}, \ldots, t_{n}\right\}
$$

Then each $\gamma(\ell)$ is a tail of $\gamma$, with $\gamma(1)=\gamma$, and similarly for $\beta$. (Note that some of the $\beta(\ell)$ may be empty, if $t_{n} \notin \beta$.)

Definition 4.2.3. For $\beta, \gamma$ as above, we define an embedding $\mathcal{A}_{\beta} \stackrel{f}{\rightarrow} \mathcal{A}_{\gamma}$ by recursively defining embeddings $\mathcal{A}_{\beta(\ell)} \stackrel{f_{\ell}}{\rightarrow} \mathcal{A}_{\gamma(\ell)}$ and letting $f=f_{1}$. The embeddings are as follows:

- In the base case $\ell=n$, the embedding $f_{n}$ is the identity map in case $t_{n} \in \beta$, or the canonical embedding $\mathbb{C} \hookrightarrow \mathcal{A}$ otherwise.

- Given $f_{\ell+1}$, let $\mathcal{B}$ denote either $\mathcal{A}$ in the case that $t_{\ell} \in \beta$, or $\mathbb{C}$ otherwise; more succinctly, $\mathcal{B}=\mathcal{A}_{\beta \cap\left\{t_{\ell}\right\}}$. Let $\mathcal{B} \stackrel{\psi}{\rightarrow} \mathcal{A}$ be either the identity map or the embedding of $\mathbb{C}$, accordingly. Then

$$
f_{\ell}=f_{\ell+1} \star \psi
$$


Proposition 4.2.4. The family of embeddings $\mathcal{A}_{\beta} \hookrightarrow \mathcal{A}_{\gamma}$ in Definition 4.2 .3 is an inductive system.

Proof. Let $\beta \leq \gamma \leq \delta$ be nonempty sets in $\mathcal{F}$. Write $\delta=\left\{t_{1}, \ldots, t_{n}\right\}$. We first prove that the embedding $\mathcal{A}_{\delta} \hookrightarrow \mathcal{A}_{\delta}$ is the identity map. We prove this for the embeddings $\mathcal{A}_{\delta(\ell)} \hookrightarrow \mathcal{A}_{\delta(\ell)}$ by reverse induction; the base case $\ell=n$ is trivial, and the inductive step is just Corollary 3.4.11.

Now for each $\ell=1, \ldots, n$ let

$$
\begin{aligned}
& \mathcal{A}_{\beta(\ell)} \stackrel{g_{\ell}}{\longrightarrow} \mathcal{A}_{\gamma(\ell)} \\
& \mathcal{A}_{\gamma(\ell)} \stackrel{f_{\ell}}{\longrightarrow} \mathcal{A}_{\delta(\ell)} \\
& \mathcal{A}_{\beta(\ell)} \stackrel{h_{\ell}}{\longrightarrow} \mathcal{A}_{\delta(\ell)}
\end{aligned}
$$

be the embeddings from Definition 4.2.3. We will prove by reverse induction for $\ell=n, \ldots, 1$ that $f_{\ell} \circ g_{\ell}=h_{\ell}$. The base case $\ell=n$ is trivial, as each of the three maps in question is either the identity map or the embedding $\mathbb{C} \hookrightarrow \mathcal{A}$. Supposing now the result to be established for $\ell+1$, let $\mathcal{B}=\mathcal{A}_{\beta \cap\left\{t_{\ell}\right\}}$ and $\mathcal{C}=\mathcal{A}_{\gamma \cap\left\{t_{\ell}\right\}}$, and let $\mathcal{B} \stackrel{\psi}{\rightarrow} \mathcal{C} \stackrel{\eta}{\rightarrow} \mathcal{A}$ be the corresponding embeddings. Then by Proposition 3.4 .13 ,

$$
h_{\ell}=h_{\ell+1} \star(\eta \circ \psi)=\left(f_{\ell+1} \circ g_{\ell+1}\right) \star(\eta \circ \psi)=\left(f_{\ell+1} \star \eta\right) \circ\left(g_{\ell+1} \star \psi\right)=f_{\ell} \circ g_{\ell} .
$$

\subsection{Endomorphisms of the Limit Algebra}

We have now constructed unital $\mathrm{C}^{*}$-algebras (resp. $\mathrm{W}^{*}$-algebras) $\mathcal{A}_{\gamma}$ for each $\gamma \in \mathcal{F}$, together with (normal) embeddings $\mathcal{A}_{\beta} \hookrightarrow \mathcal{A}_{\gamma}$ for $\beta \leq \gamma$, which we now denote 
$f_{\gamma, \beta}$, satisfying the inductive properties

$$
\begin{aligned}
& f_{\gamma, \gamma}=\operatorname{id}_{\mathcal{A}_{\gamma}} \\
& f_{\delta, \beta}=f_{\delta, \gamma} \circ f_{\gamma, \beta} \quad \text { for } \beta \leq \gamma \leq \delta .
\end{aligned}
$$

By a standard construction (see for instance section 1.23 of [Sak98], Proposition 11.4.1 of [KR86], or section II.8.2 of [Bla06]) we obtain an inductive limit, that is, a unital $\mathrm{C}^{*}$-algebra $\mathfrak{A}$ and embeddings $f_{\infty, \gamma}: \mathcal{A}_{\gamma} \rightarrow \mathfrak{A}$ such that $f_{\infty, \gamma} \circ f_{\gamma, \beta}=f_{\infty, \beta}$ for all $\beta \leq \gamma$, and with the universal property that, given any other unital $\mathrm{C}^{*}$-algebra $\mathfrak{B}$ and ${ }^{*}$-homomorphisms (not necessarily embeddings) $g_{\infty, \gamma}: \mathcal{A}_{\gamma} \rightarrow \mathfrak{B}$ satisfying $g_{\infty, \gamma} \circ f_{\gamma, \beta}=g_{\infty, \beta}$, there is a unique unital *-homomorphism $\Phi: \mathfrak{A} \rightarrow \mathfrak{B}$ satisfying $g_{\infty, \gamma}=\Phi \circ f_{\infty, \gamma}$ for all $\gamma$.

We note that inductive limits do not always exist in the category of $\mathrm{W}^{*}$ algebras and normal *-homomorphisms; hence, $\mathfrak{A}$ will not in general be a $\mathrm{W}^{*}$-algebra even when $\mathcal{A}$ is. There are a couple of standard "fixes" we could apply to replace $\mathfrak{A}$ by a suitable $W^{*}$-algebra: First, we could represent $\mathfrak{A}$ on some Hilbert space (for instance, by taking an inductive limit of appropriately intertwined representations of the $\mathcal{A}_{\gamma}$ as in [KR86] Exercise 11.5.28) and take the weak closure. However, the limit representation will in general be on a non-separable Hilbert space, which creates problems further on. Second, we could apply the double dual functor (Bla06] III.5.2, Sak98 1.17) to obtain a $\mathrm{W}^{*}$-algebra $\mathfrak{A}^{* *}$ and normal embeddings $f_{\gamma}^{* *} \circ \iota_{\gamma}: \mathcal{A}_{\gamma} \rightarrow \mathfrak{A}^{* *}$, where $\mathcal{A}_{\gamma} \stackrel{\iota_{\gamma}}{\rightarrow} \mathcal{A}_{\gamma}^{* *}$ is the canonical embedding. However, this also creates problems, as $\mathfrak{A}^{* *}$ will in general have non-separable predual even if each of the $\mathcal{A}_{\gamma}$ has separable predual. We postpone until the next chapter the question of how to adapt 
our construction to the $\mathrm{W}^{*}$-category, and continue for the time being with a purely $\mathrm{C}^{*}$-construction.

Our next task is to define a semigroup of unital *-endomorphisms of $\mathfrak{A}$. For this we note that for any $\gamma \in \mathcal{F}$ and any $\tau \geq 0$, if we let $\gamma+\tau$ denote the set $\{t+\tau \mid t \in \gamma\}$, then $\mathcal{A}_{\gamma+\tau}=\mathcal{A}_{\gamma}$. (Note that this is an equality, not just an isomorphism.) This is immediate from Definition 4.2.2 by induction on the size of $\gamma$. Similarly, $f_{\gamma+t, \beta+t}=f_{\gamma, \beta}$. But this latter equation implies that $f_{\infty, \gamma+t} \circ f_{\gamma, \beta}=f_{\infty, \beta+t}$ for any $\beta \leq \gamma$, allowing us to make the following definition.

Definition 4.3.1. For each $t \geq 0$ let $\sigma_{t}: \mathfrak{A} \rightarrow \mathfrak{A}$ denote the unique unital $*_{-}$ endomorphism obtained through the inductive limit as the unique map for which all the diagrams

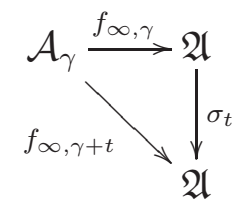

commute.

The universal property of the inductive limit then immediately implies the following.

Proposition 4.3.2. The maps $\left\{\sigma_{t}\right\}_{t \geq 0}$ form an $e_{0}$-semigroup on $\mathfrak{A}$. That $i s, \sigma_{0}=i d_{\mathfrak{A}}$, and for all $s, t \geq 0$,

$$
\sigma_{t} \circ \sigma_{s}=\sigma_{s+t} .
$$




\subsection{The Limit Retraction}

We now turn to the construction of our retraction. In the commutative ana$\operatorname{logue}$, for a set $\gamma$ with minimum time $\tau$, the retraction $\epsilon_{\gamma}$ would (when composed with the embedding $\mathcal{A} \hookrightarrow \mathfrak{A})$ correspond to a conditional expectation onto the subalgebra of $\mathfrak{A}$ consisting of functions which depend only on the location of a path at time $\tau$. This does not form a consistent system with respect to the embeddings $f_{\gamma, \beta}$, because for $\beta \leq \gamma$ one could have times in $\gamma$ earlier than any in $\beta$. However, the restriction to time sets which contain 0 is consistent, which we now show in the noncommutative case. We first consider how to relate the retraction for a given set to the retractions for its tails.

Lemma 4.4.1. Let $\gamma=\left\{t_{1}, \ldots, t_{n}\right\} \in \mathcal{F}$ and $1 \leq \ell \leq n$. Then

$$
\epsilon_{\gamma} \circ f_{\gamma, \gamma(\ell)}=\phi_{t_{\ell}-t_{1}} \circ \epsilon_{\gamma(\ell)}
$$

Proof. We proceed by (forward!) induction on $\ell$. The base case $\ell=1$ is trivial. Now supposing the result is true for $\ell$, recall that $\mathcal{A}_{\gamma(\ell)}$ is the product $\mathcal{A}_{\gamma(\ell+1)} \star \mathcal{A}$ with respect to the map $\phi_{t_{\ell+1}-t_{\ell}} \circ \epsilon_{\gamma(\ell+1)}: \mathcal{A}_{\gamma(\ell+1)} \rightarrow \mathcal{A}$, that $f_{\gamma(\ell), \gamma(\ell+1)}$ is the embedding of $\mathcal{A}_{\gamma(\ell+1)}$ into this product, and that $\epsilon_{\gamma(\ell)}$ is the Sauvageot retraction. By Equation 3.3 we therefore have

$$
\epsilon_{\gamma(\ell)} \circ f_{\gamma(\ell), \gamma(\ell+1)}=\phi_{t_{\ell+1}-t_{\ell}} \circ \epsilon_{\gamma(\ell+1)}
$$


so that

$$
\begin{aligned}
\epsilon_{\gamma} \circ f_{\gamma, \gamma(\ell+1)} & =\epsilon_{\gamma} \circ f_{\gamma, \gamma(\ell)} \circ f_{\gamma(\ell), \gamma(\ell+1)} \\
& =\phi_{t_{\ell}-t_{1}} \circ \epsilon_{\gamma(\ell)} \circ f_{\gamma(\ell), \gamma(\ell+1)} \\
& =\phi_{t_{\ell}-t_{1}} \circ \phi_{t_{\ell+1}-t_{\ell}} \circ \epsilon_{\gamma(\ell+1)} \\
& =\phi_{t_{\ell+1}-t_{1}} \circ \epsilon_{\gamma(\ell+1)} .
\end{aligned}
$$

Proposition 4.4.2. Let $\beta \leq \gamma \in \mathcal{F}$ such that the minimum time in $\gamma$ is also in $\beta$.

Then

$$
\epsilon_{\gamma} \circ f_{\gamma, \beta}=\epsilon_{\beta} .
$$

Proof. Let $\gamma=\left\{t_{1}, \ldots, t_{n}\right\}$. We will prove that

$$
\epsilon_{\gamma(\ell)} \circ f_{\gamma(\ell), \beta(\ell)}=\epsilon_{\beta(\ell)}
$$

for all $\ell$ such that $t_{\ell} \in \beta$. For the base case with the maximal such $\ell, f_{\gamma(\ell), \beta(\ell)}$ is equal to $\iota_{\gamma(\ell)}$, and since $\epsilon_{\gamma(\ell)}$ is a corresponding retraction, their composition is $\mathrm{id}_{\mathcal{A}}=\epsilon_{\beta(\ell)}$. Inductively, suppose $t_{\ell} \in \beta$ and $t_{\ell+k}$ is the next time in $\beta$, so that $\beta(\ell+1)=\beta(\ell+k)$; then

$$
\begin{aligned}
\phi_{t_{\ell+1}-t_{\ell}} \circ \epsilon_{\gamma(\ell+1)} \circ f_{\gamma(\ell+1), \beta(\ell+1)} & =\phi_{t_{\ell+1}-t_{\ell}} \circ \epsilon_{\gamma(\ell+1)} \circ f_{\gamma(\ell+1), \beta(\ell+k)} & & (\beta(\ell+1)=\beta(\ell+k)) \\
& =\phi_{t_{\ell+1}-t_{\ell}} \circ \epsilon_{\gamma(\ell+1)} \circ f_{\gamma(\ell+1), \gamma(\ell+k)} \circ f_{\gamma(\ell+k), \beta(\ell+k)} & & \text { (f's consistent) } \\
& =\phi_{t_{\ell+1}-t_{\ell}} \circ \phi_{t_{\ell+k}-t_{\ell+1}} \circ \epsilon_{\gamma(\ell+k)} \circ f_{\gamma(\ell+k), \beta(\ell+k)} & & \text { (Lemma 4.4.1) } \\
& =\phi_{t_{\ell+k}-t_{\ell}} \circ \epsilon_{\beta(\ell+k)} & & \text { (induction). }
\end{aligned}
$$


It then follows from Corollary 3.4 .12 that

$$
\epsilon_{\gamma(\ell)} \circ f_{\gamma(\ell), \beta(\ell)}=\epsilon_{\gamma(\ell)} \circ\left(f_{\gamma(\ell+1), \beta(\ell+1)} \star \mathrm{id}\right)=\epsilon_{\beta(\ell)}
$$

as desired. The case $\ell=1$ gives us the result.

Corollary 4.4.3. The restriction of the family of retractions $\left\{\epsilon_{\gamma}\right\}$ to the subset $\mathcal{F}_{0} \subset$ $\mathcal{F}$ of sets containing 0 is consistent.

Since $\mathcal{F}_{0}$ is a tail of $\mathcal{F}$, the limit $\mathfrak{A}$ is generated by images of $\mathcal{A}_{\gamma}$ with $\gamma \in \mathcal{F}_{0}$. Hence, Corollary 4.4 .3 implies the existence of a retraction $\mathbb{E}: \mathfrak{A} \rightarrow \mathcal{A}$ with the property that $\mathbb{E} \circ f_{\infty, \gamma}=\epsilon_{\gamma}$ for all $\gamma \in \mathcal{F}_{0}$.

Definition 4.4.4. The Sauvageot dilation retraction for $\left(\mathcal{A},\left\{\phi_{t}\right\}, \omega\right)$ is the map $\mathbb{E}: \mathfrak{A} \rightarrow \mathcal{A}$ characterized by

$$
\mathbb{E} \circ f_{\infty, \gamma}=\epsilon_{\gamma} \quad \text { for all } 0 \in \gamma \in \mathcal{F}
$$

We now prove that $\left(\mathbb{E},\left\{\sigma_{t}\right\}\right)$ provides a strong dilation of the semigroup $\left\{\phi_{t}\right\}$.

Theorem 4.4.5. For all $t \geq 0$,

$$
\mathbb{E} \circ \sigma_{t}=\phi_{t} \circ \mathbb{E}
$$

Proof. The case $t=0$ is trivial. Now let $\gamma \in \mathcal{F}$ be nonempty and $t>0$. Let $\delta=(\gamma+t) \cup\{0\}$; then $\mathcal{A}_{\delta}$ is the Sauvageot product $\mathcal{A}_{\gamma+t} \star \mathcal{A}$ with respect to the map $\phi_{t} \circ \epsilon_{\gamma}$. By Equation 3.3, it follows that

$$
\epsilon_{\delta} \circ f_{\delta, \gamma+t}=\phi_{t} \circ \epsilon_{\gamma}
$$


Then

$$
\begin{aligned}
\mathbb{E} \circ \sigma_{t} \circ f_{\infty, \gamma} & =\mathbb{E} \circ f_{\infty, \gamma+t} \\
& =\mathbb{E} \circ f_{\infty, \delta} \circ f_{\delta, \gamma+t} \\
& =\epsilon_{\delta} \circ f_{\delta, \gamma+t} \\
& =\phi_{t} \circ \epsilon_{\gamma} \\
& =\phi_{t} \circ \mathbb{E} \circ f_{\infty, \gamma} .
\end{aligned}
$$

So $\mathbb{E} \circ \sigma_{t}$ and $\phi_{t} \circ \mathbb{E}$ agree on the dense subalgebra of $\mathfrak{A}$ consisting of the images of all the $f_{\infty, \gamma}$; as both are contractive, they are equal.

This concludes our construction of unital $\mathrm{e}_{0}$-dilations for $\mathrm{cp}_{0}$-semigroups on $\mathrm{C}^{*}$-algebras. We summarize the result in the following theorem.

Theorem 4.4.6. Let $\mathcal{A}$ be a unital $C^{*}$-algebra on which there exists a faithful state. Then every cp $p_{0}$-semigroup on $\mathcal{A}$ has a strong unital $e_{0}$-dilation. 


\section{CHAPTER 5}

\section{CONTINUOUS W*-DILATIONS}

In the previous chapter we saw how to construct a unital $\mathrm{e}_{0}$-dilation of a $\mathrm{cp}_{0^{-}}$ semigroup. It remains to investigate whether such a construction dilates a continuous semigroup to a continuous semigroup (that is, whether it produces a unital $\mathrm{E}_{0}$-dilation of a $\mathrm{CP}_{0}$-semigroup), or, failing that, whether the construction can be modified to achieve this result. Additionally, we have not yet resolved the question of how to adapt our $\mathrm{C}^{*}$ construction to the $\mathrm{W}^{*}$ setting. To these issues we now turn our attention.

\subsection{Introduction: The Problem of Continuity}

\subsubsection{Establishing the Problem: Discontinuity of the Existing Dilation}

The first question to consider is whether the existing dilation may already be continuous. It turns out that this is never the case unless $\mathcal{A}=\mathbb{C}$. Consider a nontrivial $\mathcal{A}$ with faithful state $\omega$, and let a be any nonzero element of ker $\omega$. Fixing some faithful representation $\left(H, \Omega, \pi_{R}\right)$ of $(\mathcal{A}, \omega)$, let $h=\pi_{R}(a) \Omega$, which is orthogonal to $\Omega$. For each $t>0$ there is a faithful representation $\left(H, \Omega, \pi_{R}, K^{(t)}, V^{(t)}, \pi_{L}^{(t)}\right)$ of $\left(A, A, \phi_{t}, \omega\right)$. Form the Sauvageot product $\mathfrak{H}^{(t)}=H^{-} \star L^{(t)}$, and let $\xi$ be any unit vector in $L^{(t)}$. By Proposition 3.2 .9 we see that $\psi_{L}^{(t)}(a) \xi$ is a vector in $L^{(t)}$, whereas $\psi_{R}^{(t)}(a) \xi=h \otimes \xi$ is in $H^{-} \otimes L^{(t)}$. Since these are orthogonal subspaces of $\mathfrak{H}^{(t)}$,

$$
\left\|\psi_{L}^{(t)}(a) \xi-\psi_{R}^{(t)}(a) \xi\right\| \geq\|h \otimes \xi\|=\|h\|\|\xi\|
$$


which implies

$$
\left\|\psi_{L}^{(t)}(a)-\psi_{R}^{(t)}(a)\right\| \geq\|h\|
$$

Now letting $\gamma=\{0, t\}$, we have $\mathcal{A}_{\gamma}$ as the Sauvageot product $\mathcal{A} \star \mathcal{A}$ with respect to $\phi_{t}$, so that $\psi_{L}^{(t)}(a)-\psi_{R}^{(t)}(a)$ is the element $f_{\gamma,\{t\}}(a)-f_{\gamma,\{0\}}(a)$ of $\mathcal{A}_{\gamma}$. By the above, this element has norm at least $\|h\|$. Now because $f_{\infty, \gamma}$ is isometric,

$$
\begin{aligned}
\left\|\sigma_{t}(\iota(a))-\iota(a)\right\| & =\left\|f_{\infty,\{t\}}(a)-f_{\infty,\{0\}}(a)\right\| \\
& =\left\|f_{\infty, \gamma}\left(f_{\gamma,\{t\}}(a)-f_{\gamma,\{0\}}(a)\right)\right\| \\
& =\left\|f_{\gamma,\{t\}}(a)-f_{\gamma,\{0\}}(a)\right\| \geq\|h\| .
\end{aligned}
$$

It follows that $\left\|\sigma_{t}(\iota(a))-\iota(a)\right\| \not \rightarrow 0$ as $t \rightarrow 0^{+}$.

Upon further reflection, the discontinuity of $\left\{\sigma_{t}\right\}$ is not surprising, because it appears in the commutative dilation that the Sauvageot construction mimics. Considering again the case $\mathcal{A}=C(S), \mathfrak{A}=C(\mathscr{S})$ of Example 1.3.3. Given a regular Borel probability measure $\mu_{0}$ on $S$, we obtain via Riesz representation a regular Borel probability measure $\mu$ on $\mathscr{S}$ characterized by

$$
\forall f \in \mathfrak{A}: \quad \int_{\mathscr{S}} f d \mu=\int_{S}(\mathbb{E} f) d \mu_{0}
$$

Now consider the strong, aka point-norm, continuity of the shift semigroup. For a function $f \in \mathfrak{A}$, we want to know whether $\lim _{t \rightarrow 0^{+}}\left\|\sigma_{t} f-f\right\|=0$. We will show that in fact a less stringent form of continuity, fails, viz. the "point-pointwise" continuity defined by the property that for any fixed path $\mathfrak{p} \in \mathscr{S}$ and any $f \in \mathfrak{A},\left(\sigma_{t} f-f\right)(\mathfrak{p}) \rightarrow$ 0. The failure of point-pointwise continuity certainly implies the failure of point-norm continuity. Now let $\mathfrak{p}$ be any path not continuous at time 0 , let $\phi: S \rightarrow[0,1]$ be a 
continuous function such that $\phi(\mathfrak{p}(t)) \not \rightarrow \phi(\mathfrak{p}(0))$ as $t \rightarrow 0^{+}$(which exists by Urysohn's lemma), and let $f \in \mathfrak{A}$ be defined by $f(p)=\phi(p(0))$. Then

$$
\lim _{t \rightarrow 0^{+}}\left(\sigma_{t} f-f\right)(\mathfrak{p})=\lim _{t \rightarrow 0^{+}} \phi\left(\lambda_{t} \mathfrak{p}\right)-\phi(\mathfrak{p})=\lim _{t \rightarrow 0^{+}} \phi(\mathfrak{p}(t))-\phi(\mathfrak{p}(0)) \neq 0
$$

\subsubsection{Fixing the Problem: Skorohod Space?}

This section is not used in the rest of the thesis, but is mentioned merely for the sake of interest.

The preceding considerations show that, in the commutative setting, continuity of $\left\{\sigma_{t}\right\}$ breaks down because the path space $\mathscr{S}$ contains discontinuous paths. One could try to "repair" the construction by working instead with something like $C(\mathfrak{C})$, where $\mathfrak{C} \subset \mathscr{S}$ is the subspace of continuous paths. This runs into problems, however, because $\mathfrak{C}$ is not closed in $\mathscr{S}$ (the pointwise limit of continuous functions need not be continuous), hence not compact, so that one cannot form the commutative unital $\mathrm{C}^{*}$-algebra $C(\mathfrak{C})$. One could instead endow $\mathfrak{C}$ with the topology of uniform convergence; although it is complete in the corresponding metric, however, it is not compact nor even locally compact, so that attempts to form commutative $\mathrm{C}^{*}$-algebras such as $B C(\mathfrak{C})$ run into trouble as well.

One way out is to consider instead the Skorohod space $D$ of càdlàg paths, that is, paths which are continuous from the right and have limits from the left. Convergence in $D$ can be defined as follows: For convenience we consider paths parametrized by $[0,1)$ rather than $[0, \infty)$. Let $\Lambda$ denote the set of all continuous strictly increasing self-maps of $[0,1]$, and define $\gamma_{n} \stackrel{D}{\rightarrow} \gamma$ if there exists a sequence $\left\{\lambda_{n}\right\} \subset \Lambda$ such that 
$\lambda_{n} \rightarrow$ id uniformly and $\gamma_{n} \circ \lambda_{n} \rightarrow \gamma$ uniformly. Heuristically, this may be contrasted with uniform convergence as follows: Identifying paths with their graphs in $[0,1) \times S$, two paths are uniformly close if one may be obtained from the other by a small perturbation of the $S$ coordinates, whereas two paths are Skorohod-close if one may be obtained from the other by a simultaneous small perturbation of both the $S$ and the $[0,1)$ coordinates.

It turns out that there is a metric on $D$ which induces the aforementioned convergence, and that $D$ is separable and complete with respect to this metric ([Bil68] chapter 14, cf. [Kal97] Theorem A2.2). Furthermore, it is easy to check that translation is Skorohod continuous, that is, $\lambda_{t} \mathfrak{p} \stackrel{D}{\rightarrow} \mathfrak{p}$ as $t \rightarrow 0^{+}$for any $\mathfrak{p} \in D$. It follows that one may define the semigroup $\left\{\widehat{\sigma}_{t}\right\}$ on the commutative unital $\mathrm{C}^{*}$-algebra $C(D)$ by $\left(\widehat{\sigma}_{t} f\right)(\mathfrak{p})=f\left(\lambda_{t}(\mathfrak{p})\right)$, and that this semigroup will be point-pointwise continuous. The embedding $\widehat{i}: C(S) \rightarrow C(D)$ is defined as always by $(\widehat{i} f)(\mathfrak{p})=f(\mathfrak{p}(0))$. It remains to define a retraction $\widehat{\mathbb{E}}: C(D) \rightarrow C(S)$. For this we invoke the theorem that every Feller process has a càdlàg version ([Kal97] Theorem 17.15); that is, given a probability measure $\nu$ on $S$, one obtains a measure $\mu_{\nu}$ on $D$ such that the coordinateprojection process has $\left\{P_{t}\right\}$ as its transition semigroup. The assignment $\nu \mapsto \mu_{\nu}$ is a positive linear map from $M(S)$ to $M(D)$ which, one can verify, is weak-* continuous, and has the property that $\int_{D} \widehat{i}(f) d \mu_{\nu}=\int_{S} f d \nu$ for all $\nu \in M(S)$ and $f \in C(S)$; it is therefore the adjoint of a positive linear map $\mathbb{E}: C(D) \rightarrow C(S)$ with the property $\widehat{\mathbb{E}}(\widehat{i}(f))=f$. Furthermore, $\widehat{\mathbb{E}} \circ \widehat{\sigma}_{t} \circ \widehat{i}=P_{t}$, so that we have obtained a point-pointwise continuous dilation. 
In attempting to adapt this fix to the noncommutative setting, several obstacles present themselves:

1. What is the right notion of a "noncommutative Skorohod space"? That is, given a noncommutative $\mathrm{C}^{*}$-algebra $\mathcal{A}$, what $\mathrm{C}^{*}$-algebra bears the same relation to $\mathcal{A}$ that $C(D)$ does to $C(S)$ ?

2. What is the analogue of point-pointwise continuity in the noncommutative setting, when the elements of $\mathcal{A}$ (and of whatever algebra we dilate to) may not be functions on some state space or path space?

3. How might we obtain a theorem corresponding to the existence of càdlàg versions of Feller processes?

I do not know how to address these questions; fortunately, another approach proved successful, so that the answers to these questions are not needed in the rest of this thesis.

\subsubsection{Fixing the Problem: Dilations on $L^{\infty}$}

Another way to resolve the issue of continuity is to move from the $\mathrm{C}^{*}$ to the $\mathrm{W}^{*}$ category, by considering maps on $L^{\infty}(S)$ and $L^{\infty}(\mathscr{S})$ instead of $C(S)$ and $C(\mathscr{S})$. Given $\mathcal{A}=C(S)$ and $\mathfrak{A}=C(\mathscr{S})$ as before, we now select some regular Borel probability measure $\mu_{0}$ on $S$, and let $\widehat{\mathcal{A}}=L^{\infty}\left(S, \mu_{0}\right)$. Let $\mu$ be the corresponding measure on $\mathscr{S}$ as in Example 1.3.3, and $\widehat{\mathfrak{A}}=L^{\infty}(\mathscr{S}, \mu)$. We define the completely 
positive semigroup $\left\{\widehat{P}_{t}\right\}$ on $\mathcal{A}$ by

$$
\left(\widehat{P}_{t} f\right)(x)=\int_{S} f(y) d p_{t, x}(y)
$$

which has the additional property that each map $\widehat{P}_{t}$ is normal: If $f_{\nu} \uparrow f$ are positive elements of $\mathcal{A}$, then

$$
\left(\widehat{P}_{t} f_{\nu}\right)(x)=\int_{S} f_{\nu}(y) d p_{t, x}(y) \rightarrow \int_{S} f(y) d p_{t, x}(y)=\left(\widehat{P}_{t} f\right)(x)
$$

by Dominated Convergence.

We define the semigroup $\left\{\widehat{\sigma}_{t}\right\}$ of normal endomorphisms of $\widehat{\mathfrak{A}}$ by $\left(\widehat{\sigma}_{t} f\right)(\mathfrak{p})=$ $f\left(\lambda_{t}(\mathfrak{p})\right)$, and the normal embedding $\hat{i}: \mathcal{A} \rightarrow \mathfrak{A}$ by $(\hat{i} f)(\gamma)=f(\gamma(0))$. The image of $\hat{i}$ corresponds to $L^{\infty}\left(\mathscr{S}, \mathfrak{F}_{0}, \mu\right)$, where $\mathfrak{F}$ is the Borel $\sigma$-algebra on $\mathscr{S}$ and $\mathfrak{F}_{0} \subset \mathfrak{F}$ is the $\sigma$-subalgebra of sets of the form $E \times[0, \infty)$ for a Borel subset $E \subset S$. We therefore obtain a normal retraction $\widehat{E}: \widehat{\mathfrak{A}} \rightarrow \widehat{\mathcal{A}}$ through the Radon-Nikodym derivative, and since $\widehat{\mathbb{E}} \circ \widehat{\sigma}_{t} \circ \hat{i}$ and $\widehat{P}_{t}$ are both normal and agree on the weak-* dense subspace $C(S) \subset L^{\infty}\left(S, \mu_{0}\right)$, where they equal $\mathbb{E} \circ \sigma_{t} \circ i$ and $P_{t}$ respectively, they are equal.

We have not yet shown that $t \mapsto \widehat{\sigma}_{t}$ is weak-* continuous on $\widehat{\mathfrak{A}}$. Since we are interested in the commutative case purely for heuristic purposes at this point, we shall set aside the question of what conditions on $S, \mu_{0}$, and $\left\{P_{t}\right\}$ are necessary for some of our subsequent assumptions to hold. Let $\pi$ be the multiplication representation of $\mathcal{A}$ on $H=L^{2}\left(S, \mu_{0}\right)$, and assume that the weak closure $\pi(\mathcal{A})^{\prime \prime}$ is isomorphic to $\widehat{\mathcal{A}}$. By Stinespring dilation of $\pi \circ \mathbb{E}$ we obtain a representation $\psi$ of $\mathfrak{A}$ on some $K$ (which we could imagine to be the multiplication representation on $L^{2}(\mathscr{S}, \mu)$, but we won't use that hypothesis). We assume that the weak closure $\psi(\mathcal{A})^{\prime \prime}$ is isomorphic to $\widehat{\mathcal{A}}$. 
The semigroup $\widehat{\sigma}_{t}$ is related to $\left\{\sigma_{t}\right\}$ through the covariance relation $\widehat{\sigma}_{t} \circ \psi=\psi \circ \sigma_{t}$.

Since vectors of the form $\psi(y) V \xi$ for $y \in \mathfrak{A}, \xi \in H$ are dense in $K$, the question of WOT-continuity of $\widehat{\sigma}_{t}(a)$ reduces to the continuity of the expression

$$
\left\langle\widehat{\sigma}_{t}(a) \psi(y) V \xi, \psi(z) V \eta\right\rangle=\left\langle V^{*} \psi(z)^{*} \widehat{\sigma}_{t}(a) \psi(y) V \xi, \eta\right\rangle
$$

When we restrict to the weakly dense subset of $a \in \widehat{\mathfrak{A}}$ of the form $\psi(x)$ for $x \in \mathfrak{A}$, this expression becomes (using the covariance relation)

$$
\left\langle V^{*} \psi\left(z^{*} \sigma_{t}(x) y\right) V \xi, \eta\right\rangle=\left\langle\pi \circ \mathbb{E}\left[z^{*} \sigma_{t}(x) y\right] \xi, \eta\right\rangle
$$

We are thus led to consider the WOT-continuity properties of how the retraction $\mathbb{E}$ interactions with the translation semigroup $\sigma_{t}$. In particular, if we could find a way to reduce expressions of the form $\mathbb{E}\left[z^{*} \sigma_{t}(x) y\right]$ to expressions involving the semigroup $\widehat{P}_{t}$, we could use the assumed continuity properties of the latter. The search for such a reduction leads to the concept of moment polynomials, which we now take up.

\subsection{Moment Polynomials}

In the Sauvageot $\mathrm{C}^{*}$-dilation of chapter 4 , the inductive limit algebra $\mathfrak{A}$ is norm-generated as an algebra by elements $\sigma_{t}(i(a))$ for $t \geq 0$ and $a \in \mathcal{A}$. In studying the retraction $\mathbb{E}$, therefore, one is naturally led to consider expressions of the form

$$
\mathbb{E}\left[\sigma_{t_{1}} i\left(\left(a_{1}\right)\right) \sigma_{t_{2}}\left(i\left(a_{2}\right)\right) \ldots \sigma_{t_{n}}\left(i\left(a_{n}\right)\right)\right], \quad t_{1}, \ldots, t_{n} \geq 0 ; \quad a_{1}, \ldots, a_{n} \in \mathcal{A}
$$

In particular, it would be desirable to have a formula for the value of (5.1) in terms of the original semigroup $\left\{\phi_{t}\right\}$ and the state $\omega$ chosen for the dilation procedure. From 
the construction of $\mathbb{E}$ in previous chapters, we see that (5.1) can be evaluated as follows:

- If all the $t_{i}$ are strictly positive, let $\tau$ denote the minimum; then, by Theorem 4.4 .5 ,

$$
\mathbb{E}\left[\sigma_{t_{1}} i\left(\left(a_{1}\right)\right) \sigma_{t_{2}}\left(i\left(a_{2}\right)\right) \cdots \sigma_{t_{n}}\left(i\left(a_{n}\right)\right)\right]=\phi_{\tau}\left(\mathbb{E}\left[\sigma_{t_{1}-\tau} i\left(\left(a_{1}\right)\right) \sigma_{t_{2}-\tau}\left(i\left(a_{2}\right)\right) \cdots \sigma_{t_{n}-\tau}\left(i\left(a_{n}\right)\right)\right]\right)
$$

- If some of the $t_{i}$ are zero, let $\tau$ be the minimum of the nonzero values, and let $\gamma+\tau$ be the set of nonzero values, where $\gamma$ is a finite subset of $[0, \infty)$. Then $\sigma_{t_{1}}\left(i\left(a_{1}\right)\right) \cdots \sigma_{t_{n}}\left(i\left(a_{n}\right)\right)$ may be viewed as an element of $\mathcal{A}_{\{0\} \cup(\gamma+\tau)}$, which we further break down as a word in $\mathcal{A}$ and $\mathcal{A}_{\gamma}$, related through the map $\mathcal{A}_{\gamma} \stackrel{\phi_{\tau} \circ \epsilon_{\gamma}}{\rightarrow} \mathcal{A}$; we apply the right-liberation property to calculate the value of $\mathbb{E}$ on this word.

As in the second chapter, we now formalize this strategy in terms of recursively defined functions.

\section{Notation 5.2.1.}

- For $n \geq 1$ let $[0, \infty)_{0}^{n}$ denote the subset

$$
\left\{\left(t_{1}, \ldots, t_{n}\right) \in[0, \infty)^{n} \mid \min \left(t_{1}, \ldots, t_{n}\right)=0\right\}
$$

- For $n \geq 1$ let $\psi_{n}:[0, \infty)^{n} \rightarrow[0, \infty) \times[0, \infty)_{0}^{n}$ denote the homeomorphism

$$
\psi_{n}\left(t_{1}, \ldots, t_{n}\right)=\left(\min t_{i}, t_{1}-\min t_{i}, \ldots, t_{n}-\min t_{i}\right)
$$

with inverse

$$
\psi_{n}^{-1}\left(\tau, s_{1}, \ldots, s_{n}\right)=\left(s_{1}+\tau, \ldots, s_{n}+\tau\right) .
$$


- For $n \geq 1, \vec{s} \in[0, \infty)_{0}^{n}$, and $\vec{a} \in \mathcal{A}^{n}$, the standard decomposition of $(\vec{s}, \vec{a})$ is as follows: Write $\vec{s}=\overrightarrow{\mathfrak{n}}_{0} \vee \overrightarrow{\mathfrak{s}}_{0} \vee \overrightarrow{\mathfrak{n}}_{1} \vee \cdots \vee \overrightarrow{\mathfrak{s}}_{m-1} \vee \overrightarrow{\mathfrak{n}}_{m}$, where each entry in each $\overrightarrow{\mathfrak{n}}_{k}$ is zero, each entry in each $\overrightarrow{\mathfrak{s}}_{k}$ is nonzero, and some of $\overrightarrow{\mathfrak{n}}_{0}, \overrightarrow{\mathfrak{s}}_{0}$, and $\overrightarrow{\mathfrak{n}}_{m}$ may be empty; here $\vee$ denotes concatenation. Write $\vec{a}=\vec{z}_{0} \vee \vec{w}_{0} \vee \vec{z}_{1} \vee \cdots \vee \vec{w}_{m-1} \vee \vec{z}_{m}$, where each $\vec{z}_{i}$ has the same length as $\overrightarrow{\mathfrak{n}}_{i}$ and each $\vec{w}_{i}$ the same length as $\overrightarrow{\mathfrak{s}}_{i}$. We refer to $\left(\overrightarrow{\mathfrak{n}}_{0}, \overrightarrow{\mathfrak{s}}_{0}, \ldots, \overrightarrow{\mathfrak{n}}_{m}\right)$ as the standard decomposition of $(\vec{s})$, and $\left(\vec{z}_{0}, \vec{w}_{0}, \ldots, \vec{z}_{m}\right)$ as the standard decomposition of $\vec{a}$ with respect to $\vec{s}$. The alternation number of $\vec{s}$, denoted alt $(\vec{s})$, is the number $m$ appearing in the standard decomposition; the alternation number of an element $\vec{t} \in[0, \infty)^{n}$ is the alternation number of $\psi_{n}(\vec{t})$.

- By $\left([0, \infty)_{0} \times \mathcal{A}\right)_{L}^{\sharp}$ we denote the set of tuples $(\vec{s}, \vec{a}, \vec{\iota})$ such that, for some $n \geq 1$, $\vec{s} \in[0, \infty)_{0}^{n}, \vec{a} \in \mathcal{A}^{n}$, and $\vec{\iota} \in[\operatorname{alt}(\vec{s})-1]$, with the convention $[-1]=\emptyset$. By $\left([0, \infty)_{0} \times \mathcal{A}\right)_{R}^{\sharp}$ we denote the same set except with $[\operatorname{alt}(\vec{s})]$ in place of $[\operatorname{alt}(\vec{s})-1]$.

We next introduce "diachronic" versions of the collapse and moment functions from chapter 2 . 
Definition 5.2.2. We recursively define functions

$$
\begin{gathered}
\mathfrak{S}:([0, \infty) \times \mathcal{A})^{\sharp} \rightarrow \mathcal{A} \\
\operatorname{DRM}:([0, \infty) \times \mathcal{A})^{\sharp} \rightarrow \mathcal{A} \\
\operatorname{DUM}:([0, \infty) \times \mathcal{A})^{\sharp} \rightarrow \mathcal{A} \\
\operatorname{DRC}:([0, \infty) \times \mathcal{A})_{R}^{\sharp} \rightarrow([0, \infty) \times \mathcal{A})^{\sharp} \\
\operatorname{DLC}:([0, \infty) \times \mathcal{A})_{L}^{\sharp} \rightarrow([0, \infty) \times \mathcal{A})^{\sharp} \\
\text { DUC }:([0, \infty) \times \mathcal{A})_{L}^{\sharp} \rightarrow([0, \infty) \times \mathcal{A})^{\sharp}
\end{gathered}
$$

as follows:

1. $\mathfrak{S}(t ; a)=\operatorname{DRM}(t ; a)=\operatorname{DUM}(t ; a)=\phi_{t}(a)$.

2. For $n \geq 2, \vec{t} \in[0, \infty)^{n}$, and $\vec{a} \in \mathcal{A}^{n}$, let $m=\operatorname{alt}(\vec{t})$; then

$$
\begin{aligned}
\mathfrak{S}(\vec{t} ; \vec{a}) & =\sum_{\vec{\imath} \subset[m-1]} \operatorname{DRM}(\operatorname{DLC}(\vec{t} ; \vec{a} ; \vec{\imath})) \prod_{j \in[m-1] \backslash \vec{\iota}} \omega\left(\Pi\left(\vec{z}_{2 j+1}\right)\right) \\
\operatorname{DRM}(\vec{t} ; \vec{a}) & =\sum_{\vec{\iota} \subsetneq[m]} \operatorname{DUM}(\operatorname{DRC}(\vec{t} ; \vec{a} ; \vec{\iota})) \\
\operatorname{DUM}(\vec{t} ; \vec{a}) & =\sum_{\vec{\iota} \subset[m]} \mathfrak{S}(\operatorname{DUC}(\vec{t} ; \vec{a} ; \vec{\iota}))
\end{aligned}
$$

3. For $\vec{t}, \vec{a}$ as above, let $(\tau, \vec{s})=\psi_{n}(\vec{t}),\left(\overrightarrow{\mathfrak{n}}_{0}, \ldots, \overrightarrow{\mathfrak{n}}_{m}\right)$ be the standard decomposition of $\vec{s}$, and $\left(\vec{z}_{0}, \ldots, \vec{z}_{m}\right)$ the corresponding standard decomposition of $\vec{a}$. Given also $\vec{\iota} \subset[m-1]$, let $\vec{\iota}=\left(i_{1}, \ldots, i_{\ell}\right)$. For each $k=1, \ldots, \ell+1$ define $\vec{\alpha}_{k}=$ $\vec{w}_{i_{k-1}+1} \vee \cdots \vee \vec{w}_{i_{k}}$, with the conventions $i_{0}=0$ and $i_{\ell+1}=m$, and corresponding time vectors $\vec{u}_{k}=\overrightarrow{\mathfrak{s}}_{i_{k-1}+1} \vee \cdots \vee \overrightarrow{\mathfrak{s}}_{i_{k}}$. Let

$$
\overrightarrow{\mathfrak{u}}=\overrightarrow{\mathfrak{n}}_{0} \vee \vec{u}_{1} \vee\{0\} \vee \vec{u}_{2} \vee\{0\} \vee \cdots \vee\{0\} \vee \vec{u}_{\ell+1} \vee \overrightarrow{\mathfrak{n}}_{m+1}
$$


and

$\vec{b}=\vec{z}_{0} \vee \vec{\alpha}_{1} \vee\left\{\Pi\left(\vec{z}_{i_{1}}\right)-\omega\left(\Pi\left(\vec{z}_{i_{1}}\right)\right)\right\} \vee \cdots \vee\left\{\Pi\left(\vec{z}_{i_{\ell}}\right)-\omega\left(\Pi\left(\vec{z}_{i_{\ell}}\right)\right)\right\} \vee \vec{\alpha}_{\ell+1} \vee \vec{z}_{m+1}$.

Then $\operatorname{DLC}(\vec{t} ; \vec{a})=(\overrightarrow{\mathfrak{u}} ; \vec{b})$.

4. For $\vec{t}, \vec{a}$ as above and $\vec{\iota}=\left(i_{1}, \ldots, i_{\ell}\right) \subset[m]$, with $i_{0}=0$ and $i_{\ell+1}=m+1$, define for each $k=0, \ldots, \ell$ the elements

$$
\begin{aligned}
& \beta_{k}=\Pi\left(\vec{z}_{i_{k}}\right) \prod_{j=i_{k}+1}^{i_{k+1}-1} \mathfrak{S}\left(\overrightarrow{\mathfrak{s}}_{j} ; \vec{w}_{j}\right) \Pi\left(\vec{z}_{j}\right) \\
& \gamma_{k}=\Pi\left(\vec{z}_{i_{k}}\right) \prod_{j=i_{k}+1}^{i_{k+1}-1}-\mathfrak{S}\left(\overrightarrow{\mathfrak{s}}_{j} ; \vec{w}_{j}\right) \Pi\left(\vec{z}_{j}\right)
\end{aligned}
$$

Let

$$
\begin{aligned}
& \overrightarrow{\mathfrak{u}}=\{0\} \vee \overrightarrow{\mathfrak{s}}_{i_{1}} \vee\{0\} \vee \overrightarrow{\mathfrak{s}}_{i_{2}} \vee \cdots \vee \overrightarrow{\mathfrak{s}}_{i_{m}} \vee\{0\} \\
& \vec{b}=\left\{\beta_{0}\right\} \vee \vec{w}_{i_{1}} \vee\left\{\beta_{1}\right\} \vee \cdots \vee \vec{w}_{i_{\ell}} \vee\left\{\beta_{\ell}\right\} \\
& \vec{c}=\left\{\gamma_{0}\right\} \vee \vec{w}_{i_{1}} \vee\left\{\gamma_{1}\right\} \vee \cdots \vee \vec{w}_{i_{\ell}} \vee\left\{\gamma_{\ell}\right\}
\end{aligned}
$$

Then

$$
\begin{aligned}
& \operatorname{DRC}(\vec{t} ; \vec{a} ; \vec{\iota})=(\overrightarrow{\mathfrak{u}} ; \vec{b}) \\
& \operatorname{DUC}(\vec{t} ; \vec{a} ; \vec{\iota})=(\overrightarrow{\mathfrak{u}} ; \vec{c}) .
\end{aligned}
$$

We note that the DLC, DRC, and DUC functions output vector pairs at least as short as the input vector pairs; this together with the strict subset inclusion in the definition of DRM yield a well-defined recursion from the above formulas.

The reason for defining these functions is the following proposition: 
Proposition 5.2.3. Let $\mathcal{A}$ be a unital $C^{*}$-algebra, $\left\{\phi_{t}\right\}$ a $C P_{0}$-semigroup on $\mathcal{A}$, $\omega$ a faithful state on $\mathcal{A}$, and $\left(\mathfrak{A}, i, \mathbb{E},\left\{\sigma_{t}\right\}\right)$ the Sauvageot dilation. Then for every $t_{1}, \ldots, t_{n} \geq 0$ and $a_{1}, \ldots, a_{n} \in \mathcal{A}$

$$
\mathbb{E}\left[\sigma_{t_{1}}\left(i\left(a_{1}\right)\right) \cdots \sigma_{t_{n}}\left(i\left(a_{n}\right)\right)\right]=\mathfrak{S}(\vec{t} ; \vec{a})
$$

Proof. Recall from Definition 4.4.4 that $\mathbb{E} \circ f_{\infty, \gamma}=\epsilon_{\gamma}(a)$ for any $\gamma \in \mathcal{F}$. Here, letting $\gamma$ be the union of $\vec{t}, \gamma(2)$ the immediate tail of $\gamma$ with distance $\tau$, and defining the elements $\mathfrak{a}_{i} \in \mathcal{A}_{\gamma(2)}$ corresponding to times $\overrightarrow{\mathfrak{s}}_{i}$ and tuples $\vec{w}_{i}$, the above expectation is, by Definition 4.2.2, Corollary 3.3.8, and Theorem 2.4.2, equal to

$$
\operatorname{LM}\left(\Pi\left(\vec{z}_{0}\right), \mathfrak{a}_{1}, \Pi\left(\vec{z}_{1}\right), \ldots, \mathfrak{a}_{m}, \Pi\left(\vec{z}_{m+1}\right) ; \phi_{\tau} \circ \epsilon_{\gamma(2)}\right)
$$

Further consideration of what happens when $\epsilon_{\gamma(2)}$ is applied to the elements $\mathfrak{a}_{i}$, together with the recurrence that defines the LM function, shows that $\mathbb{E}\left[\sigma_{t_{1}}\left(i\left(a_{1}\right)\right) \cdots \sigma_{t_{n}}\left(i\left(a_{n}\right)\right)\right]$ and $\mathfrak{S}(\vec{t} ; \vec{a})$ satisfy the same recurrence and initial conditions, so they are equal.

\subsection{Continuity Properties of Moment Polynomials}

The continuity properties of $\mathfrak{S}(\vec{t} ; \vec{a})$ in the case where $\mathcal{A}$ is a $\mathrm{W}^{*}$-algebra will be important in what follows. There are three types of continuity properties to consider: continuity in $a_{1}, \ldots, a_{n}$ with respect to both the weak and the strong topologies, and continuity in $t_{1}, \ldots, t_{n}$. It turns out that weak continuity holds with respect to $a_{1}, \ldots, a_{n}$ separately (which is the best we could hope for, as multiplication is not jointly weakly continuous), whereas strong continuity holds jointly in $a_{1}, \ldots, a_{n}$, and a restricted form of joint continuity in $t_{1}, \ldots, t_{n}$ holds as well. 
Proposition 5.3.1. Let $\mathcal{A}$ be a $W^{*}$-algebra, $\left\{\phi_{t}\right\}$ a $C P_{0}$-semigroup on $\mathcal{A}, \omega$ a faithful normal state on $\mathcal{A}$. Fix $n \geq 1, t_{1}, \ldots, t_{n} \geq 0, j \in\{1, \ldots, n\}$, and $a_{k}$ for $k \in$ $\{1, \ldots, n\} \backslash\{j\}$. Then $\mathfrak{S}(\vec{t} ; \vec{a})$, viewed as a function of $a_{j}$, is a normal linear map from $\mathcal{A}$ to itself.

Proof. This is a straightforward induction from Definition (5.2.2); we show simultaneously that the six functions $\mathfrak{S}$, DRM, DUM, DLC, DRC, DUC are normal functions of $a_{j}$ when the other $a_{i}$ and all the $t_{i}$ are fixed. This follows from the normality of the state $\omega$ and the maps $\phi_{t}$, as well as the normality of multiplication by a fixed element of $\mathcal{A}$.

Definition 5.3.2. For $n \geq 1$ and elements $\left\{\vec{s}_{k}\right\}$ and $\vec{t}$ of $[0, \infty)^{n}$, we say that $\vec{s}_{k}$ converges non-crossingly to $\vec{t}$ if $\vec{s}_{k} \rightarrow \vec{t}$ and, for all $k$, the order relations among the entries of $\vec{s}_{k}$ are the same as those in $\vec{t}$; that is, if

$$
\forall k: \forall i, j=1, \ldots, n:\left(s_{k}\right)_{i} \leq\left(s_{k}\right)_{j} \quad \Leftrightarrow \quad t_{i} \leq t_{j}
$$

Proposition 5.3.3. Let $\mathcal{A}$ be a separable $W^{*}$-algebra, $\left\{\phi_{t}\right\}$ a $C P_{0}$-semigroup on $\mathcal{A}, \omega$ a faithful normal state on $\mathcal{A}$. Let $n \geq 1$. Let $\vec{t}_{k} \rightarrow \vec{t}$ converge non-crossingly, and let $\vec{a}_{k} \rightarrow \vec{a}$ be a strongly convergent sequence of tuples in $\left(\mathcal{A}_{1}\right)^{n}$. Then $\mathfrak{S}\left(\vec{t}_{k} ; \vec{a}_{k}\right) \rightarrow \mathfrak{S}(\vec{t} ; \vec{a})$ strongly. That is, $\mathfrak{S}(\vec{t} ; \vec{a})$ is jointly strongly continuous in $\vec{t}$ and $\vec{a}$, subject to the noncrossing restriction on $\vec{t}$.

Proof. We induct on $n$, using definition (5.2.2) and the following observations:

- If $\vec{t}_{k} \rightarrow \vec{t}$ non-crossingly, then $\vec{s}_{k} \rightarrow \vec{s}$ non-crossingly, where $\vec{s}_{k}, \vec{s}$ are the subtuples of $\vec{t}_{k}, \vec{t}$ corresponding to a fixed subset of indices from $\{1, \ldots, n\}$. 
- If $\vec{t}_{k} \rightarrow \vec{t}$ non-crossingly and $\vec{\imath}$ is given, let $\overrightarrow{\mathfrak{u}}$ be defined from $\vec{t}$ and $\overrightarrow{\mathfrak{u}}_{k}$ from $\vec{t}_{k}$ as in the DRC and DUC functions; then $\overrightarrow{\mathfrak{u}}_{k} \rightarrow \overrightarrow{\mathfrak{u}}$ non-crossingly.

- If $\vec{t}_{k} \rightarrow \vec{t}$ non-crossingly, then $\psi_{n}\left(\vec{t}_{k}\right)$ and $\psi_{n}(\vec{t})$ are zero at the same entries; moreover, the corresponding parts $\overrightarrow{\mathfrak{n}}_{i}, \overrightarrow{\mathfrak{s}}_{i}$ of the standard decompositions of $\vec{t}_{k}$ and of $\vec{t}$ are all the same length.

These considerations plus the strong continuity of the state $\omega$ and the joint strong continuity of the semigroup $\phi$ (Theorem 1.4.2) imply the result.

\subsection{The Continuous Theorem}

We now return to the question of how to obtain a continuous $\mathrm{W}^{*}$-dilation from an algebraic $\mathrm{C}^{*}$-dilation. The technique in this section is adapted from the eighth chapter of $\underline{\operatorname{Arv} 03}$. Throughout, we let $\mathcal{A}$ denote a separable $\mathrm{W}^{*}$-algebra, $\left\{\phi_{t}\right\}$ a $\mathrm{CP}_{0^{-}}$-semigroup on $\mathcal{A},\left(\mathfrak{A}, i, \mathbb{E},\left\{\sigma_{t}\right\}\right)$ the Sauvageot dilation from the previous chapter, $\mathcal{P} \subset \mathfrak{A}$ the subset

$$
\mathcal{P}=\left\{\sigma_{t_{1}}\left(i\left(a_{1}\right)\right) \ldots \sigma_{t_{k}}\left(i\left(a_{k}\right)\right) \mid t_{1}, \ldots, t_{k} \geq 0 ; a_{1}, \ldots, a_{k} \in \mathcal{A}\right\}
$$

$\mathfrak{A}_{0} \subseteq \mathfrak{A}$ the norm-dense linear span of $\mathcal{P},(H, \pi)$ a faithful normal representation of $\mathcal{A}$ on a separable Hilbert space, $(\mathfrak{H}, V, \psi)$ a minimal Stinespring dilation of $\pi \circ \mathbb{E}$, $\widetilde{\mathfrak{A}}=\psi(\mathfrak{A})^{\prime \prime}$, and $\widetilde{\mathbb{E}}: \widetilde{\mathfrak{A}} \rightarrow \mathcal{A}$ the map $\widetilde{\mathbb{E}}[T]=\pi^{-1}\left(V^{*} T V\right)$, which is well-defined because $T \mapsto V^{*} T V$ is normal and maps the weakly dense subspace $\psi(\mathfrak{A}) \subset \widetilde{\mathfrak{A}}$ into the weakly closed set $\pi(\mathcal{A})$, and because $\pi$ is faithful; it satisfies $\widetilde{\mathbb{E}} \circ \psi=\mathbb{E}$ and therefore is a normal retraction with respect to $\psi \circ i$. 
We begin with the observation that weak-operator continuity of families of contractions can be checked on a dense subset of Hilbert space.

Lemma 5.4.1. Let $\mathcal{H}$ be a Hilbert space and $\left\{T_{t}\right\}_{t \geq 0}$ a family (not necessarily a semigroup) of contractions on $\mathcal{H}$. Let $\mathcal{H}_{0} \subseteq \mathcal{H}$ be a dense linear subspace such that for all $\xi, \eta \in \mathcal{H}_{0}$, the map $t \mapsto\left\langle T_{t} \xi, \eta\right\rangle$ is continuous. Then $t \mapsto T_{t}$ is WOT-continuous.

Proof. Let $\xi, \eta \in \mathcal{H}$ and $t_{0} \geq 0$. Given $\epsilon>0$, choose $\xi_{0}, \eta_{0} \in \mathcal{H}_{0}$ with $\left\|\xi-\xi_{0}\right\|<$ $\max (1, \epsilon)$ and $\left\|\eta-\eta_{0}\right\|<\epsilon$. Then for any $t \geq 0$,

$$
\begin{aligned}
\left\langle\left(T_{t}-T_{t_{0}}\right) \xi, \eta\right\rangle & =\left\langle\left(T_{t}-T_{t_{0}}\right) \xi_{0}, \eta_{0}\right\rangle \\
& +\left\langle\left(T_{t}-T_{t_{0}}\right) \xi_{0}, \eta-\eta_{0}\right\rangle \\
& +\left\langle\left(T_{t}-T_{t_{0}}\right)\left(\xi-\xi_{0}\right), \eta\right\rangle .
\end{aligned}
$$

The first term tends to zero as $t \rightarrow t_{0}$ by hypothesis, so that in particular it is less than $\epsilon$ for $t$ sufficiently close to $t_{0}$. The second term is at most $2\left\|\xi_{0}\right\| \epsilon \leq 2(\|\xi\|+1) \epsilon$ by Cauchy-Schwarz, and the third term at most $2\|\eta\| \epsilon$. Hence

$$
\left|\left\langle\left(T_{t}-T_{t_{0}}\right) \xi, \eta\right\rangle\right| \leq(3+2\|\eta\|+2\|\xi\|) \epsilon
$$

for $t$ sufficiently near $t_{0}$.

The next lemma is rather technical, but it advances our study of how $\mathbb{E}$ interacts with time translations, and in particular with translation of the middle term of a threefold product. 
Lemma 5.4.2. Let $y, z \in \mathcal{P}$ and $t \geq 0$. There exist $y_{0}, z_{0} \in \mathcal{P}$ and a normal linear map $Q: \mathcal{A} \rightarrow \mathcal{A}$ such that, for every $x \in \mathfrak{A}$,

$$
\mathbb{E}\left[y \sigma_{t}(x) z\right]=Q\left(\mathbb{E}\left[y_{0} x z_{0}\right]\right)
$$

Proof. Let $y=\sigma_{s_{1}}\left(a_{1}\right) \ldots \sigma_{s_{m}}\left(a_{m}\right)$ and $z=\sigma_{t_{1}}\left(b_{1}\right) \ldots \sigma_{t_{n}}\left(b_{n}\right)$. We proceed by strong induction on $m+n$. In the base case $m+n=0$ (meaning that $y=z=1$ ) the requisite map is $Q=\phi_{t}$, by Theorem 4.4.5. Inductively, letting $\tau=\min \left(s_{1}, \ldots, s_{m}, t_{1}, \ldots, t_{n}\right)$, the result is again trivial in case $t \leq \tau$, as then one can use $Q=\phi_{t}, y_{0}=\sigma_{s_{1}-t}\left(a_{1}\right) \cdots \sigma_{s_{m}-t}\left(a_{m}\right)$, and $z_{0}=\sigma_{t_{1}-t}\left(b_{1}\right) \cdots \sigma_{t_{n}-t}\left(b_{n}\right)$. Hence we assume $t>\tau$. We further assume $\tau=0$, as the case $\tau>0$ reduces to this by Theorem 4.4.5 again.

Let $\left(s_{1}^{\prime}, \ldots, s_{q}^{\prime}\right)$ be the (possibly empty) final segment of nonzero entries from $\left(s_{1}, \ldots, s_{m}\right)$, and $\left(a_{1}^{\prime}, \ldots, a_{q}^{\prime}\right)$ the corresponding entries from $\left(a_{1}, \ldots, a_{m}\right)$. Similarly, let $\left(t_{1}^{\prime}, \ldots, t_{p}^{\prime}\right)$ be the initial segment of nonzero entries from $\left(t_{1}, \ldots, t_{n}\right)$, and $\left(b_{1}^{\prime}, \ldots, b_{p}^{\prime}\right)$ the corresponding entries from $\left(b_{1}, \ldots, b_{n}\right)$. Let $y_{0}=\sigma_{s_{1}^{\prime}}\left(a_{1}^{\prime}\right) \ldots \sigma_{s_{q}^{\prime}}\left(a_{q}^{\prime}\right)$ and $z_{0}=$ $\sigma_{t_{1}^{\prime}}\left(b_{1}^{\prime}\right) \ldots \sigma_{t_{p}^{\prime}}\left(b_{p}^{\prime}\right)$

For any $x \in \mathcal{P}$, write $x=\sigma_{u_{1}}\left(c_{1}\right) \cdots \sigma_{u_{\ell}}\left(c_{\ell}\right)$, so that $\sigma_{t}(x)=\sigma_{u_{1}+t}\left(c_{1}\right) \cdots \sigma_{u_{\ell}+t}\left(c_{\ell}\right)$. Now $\mathbb{E}\left[y \sigma_{t}(x) z\right]=\mathfrak{S}(\vec{s} \vee(\vec{u}+t) \vee \vec{t} ; \vec{a} \vee \vec{c} \vee \vec{b})$ by Proposition 5.2.3, In the standard decomposition $\vec{s} \vee(\vec{u}+t) \vee \vec{t}=\overrightarrow{\mathfrak{n}}_{1} \vee \overrightarrow{\mathfrak{s}}_{1} \vee \cdots \vee \overrightarrow{\mathfrak{n}}_{m+1}$, we must have $\vec{u}+t$ contained in a single one of the $\overrightarrow{\mathfrak{s}}_{i}$; more specifically, for some $i$ we have $\overrightarrow{\mathfrak{s}}_{i}=\left(s_{1}^{\prime}, \ldots, s_{q}^{\prime}\right) \vee(\vec{u}+t) \vee\left(t_{1}^{\prime}, \ldots, t_{p}^{\prime}\right)$ and $\vec{w}_{i}=\left(a_{1}^{\prime}, \ldots, a_{q}^{\prime}\right) \vee \vec{c} \vee\left(b_{1}^{\prime}, \ldots, b_{p}^{\prime}\right)$. Then Propositions (5.2.3) and (2.4.6) imply that $\mathbb{E}\left[y \sigma_{t}(x) z\right]$ is the composition of $\mathbb{E}\left[y_{0} x z_{0}\right]$ with some normal map $Q$, which is independent of $x$. This gives us equation (5.2) for all $x \in \mathcal{P}$, and since both sides are linear and norm-continuous in $x$, it follows that (5.2) holds for all $x \in \mathfrak{A}$. 
Theorem 5.4.3. There exists a (necessarily unique) semigroup of normal unital *endomorphisms $\left\{\widetilde{\sigma}_{t}\right\}_{t \geq 0}$ of $\widetilde{\mathfrak{A}}$ such that

$$
\forall t \geq 0: \quad \tilde{\sigma}_{t} \circ \psi=\psi \circ \sigma_{t} .
$$

Proof. We construct $\left\{\widetilde{\sigma}_{t}\right\}$ and verify its properties in the following sequence of steps.

1. For each $t \geq 0$ and $\xi, \eta \in \psi(\mathcal{P}) V H$, we construct a normal linear functional $\rho_{t, \xi, \eta}$ on $\widetilde{\mathfrak{A}}$ as follows. Let $\xi=\psi(y) V \xi^{\prime}$ and $\eta=\psi(z) V \eta^{\prime}$ for $y, z \in \mathcal{P}$ and $\xi^{\prime}, \eta^{\prime} \in H$. By Lemma (5.4.2), there exists a normal linear map $Q: \mathcal{A} \rightarrow \mathcal{A}$ and elements $y_{0}, z_{0} \in \mathcal{P}$ such that $\mathbb{E}\left[z^{*} \sigma_{t}(x) y\right]=Q\left(\mathbb{E}\left[z_{0}^{*} x y_{0}\right]\right)$ for all $x \in \mathfrak{A}$. We thus have

$$
\forall x \in \mathfrak{A}: \quad\left\langle\psi\left(\sigma_{t}(x)\right) \xi, \eta\right\rangle=\left\langle\pi \circ Q \circ \mathbb{E}\left[z_{0}^{*} x y_{0}\right] \xi^{\prime}, \eta^{\prime}\right\rangle
$$

We now define $\rho_{t, \xi, \eta}$ by

$$
\rho_{t, \xi, \eta}(T)=\left\langle\pi \circ Q \circ \widetilde{\mathbb{E}}\left[\psi\left(z_{0}\right)^{*} T \psi\left(y_{0}\right)\right] \xi^{\prime}, \eta^{\prime}\right\rangle, \quad T \in \widetilde{\mathfrak{A}} .
$$

Then that the restriction to $\psi(\mathfrak{A})$ satisfies

$$
\begin{aligned}
\forall x \in \mathfrak{A}: \quad \rho_{t, \xi, \eta}(\psi(x)) & =\left\langle\pi \circ Q \circ \widetilde{\mathbb{E}} \circ \psi\left(z_{0}^{*} x y_{0}\right) \xi^{\prime}, \eta^{\prime}\right\rangle \\
& =\left\langle\pi \circ Q \circ \mathbb{E}\left[z_{0}^{*} x y_{0}\right] \xi^{\prime}, \eta^{\prime}\right\rangle \\
& =\left\langle\pi \circ \mathbb{E}\left[z^{*} \sigma_{t}(x) y\right] \xi^{\prime}, \eta^{\prime}\right\rangle \\
& =\left\langle V^{*} \psi\left(z^{*} \sigma_{t}(x) y\right) V \xi^{\prime}, \eta^{\prime}\right\rangle \\
& =\left\langle\psi\left(\sigma_{t}(x)\right) \xi, \eta\right\rangle .
\end{aligned}
$$

2. We extend the definition to $\xi, \eta$ in the linear span of $\psi(\mathcal{P}) V H$ in the natural way; for $\xi=\sum_{i} c_{i} \xi_{i}$ and $\eta=\sum_{j} d_{j} \eta_{j}$ with $\xi_{i}, \eta_{j} \in \psi(\mathcal{P}) V H$, we define $\rho_{t, \xi, \eta}=$ 
$\sum_{i, j} c_{i} \bar{d}_{j} \rho_{t, \xi_{i}, \eta_{j}}$. This is well-defined because, if $\sum_{i} c_{i} \xi_{i}=\sum_{k} \tilde{c}_{k} \tilde{\xi}_{k}$ and $\sum_{j} d_{j} \eta_{j}=$ $\sum_{\ell} \tilde{d}_{\ell} \tilde{\eta}_{\ell}$ then equation (5.4) implies that, for $x$ in the ultraweakly dense subspace $\psi(\mathfrak{A})$ of $\widetilde{\mathfrak{A}}$,

$$
\begin{aligned}
\rho_{t, \sum c_{i} \xi_{i}, \sum d_{j} \eta_{j}}(\psi(x)) & =\left\langle\psi\left(\sigma_{t}(x)\right) \sum c_{i} \xi_{i}, \sum d_{j} \eta_{j}\right\rangle \\
& =\left\langle\psi\left(\sigma_{t}(x)\right) \sum \tilde{c}_{k} \tilde{\xi}_{k}, \sum \tilde{d}_{\ell} \tilde{\eta}_{\ell}\right\rangle=\rho_{t, \sum \tilde{c}_{k} \tilde{\xi}_{k}, \sum \tilde{d}_{\ell} \tilde{n}_{\ell}}(\psi(x)) .
\end{aligned}
$$

3. Next, we note that equation (5.4) also implies that $\left\|\rho_{t, \xi, \eta}\right\| \leq\|\xi\|\|\eta\|$. This allows us to extend the definition to $\xi, \eta$ in the norm closure of the linear span of $\psi(\mathcal{P}) V H$, which is all of $\mathfrak{H}$.

4. Having defined the family of functionals $\left\{\rho_{t, \xi, \eta}\right\}$, we now use them to define the family of endomorphisms $\left\{\widetilde{\sigma}_{t}\right\}$. Equation (5.4) implies that, for fixed $t \geq 0$ and $x \in \mathfrak{A}, \rho_{\xi, \eta}(\psi(x))$ is a bounded sesquilinear function of $\xi$ and $\eta$, so that it corresponds to a unique operator in $B(\mathfrak{H})$, which we call $S_{t}(\psi(x))$, characterized by the property

$$
\forall \xi, \eta \in \mathfrak{H}: \quad \rho_{t, \xi, \eta}(\psi(x))=\left\langle S_{t}(\psi(x)) \xi, \eta\right\rangle .
$$

5. Equations (5.4) and (5.5) together imply that

$$
\forall x \in \mathfrak{A}: \quad S_{t}(\psi(x))=\psi\left(\sigma_{t}(x)\right) .
$$

6. Because $\psi$ and $\sigma_{t}$ are unital ${ }^{*}$-homomorphisms, equation (5.6) implies that $S_{t}$ is as well. 
7. Because $S_{t}$ is a unital ${ }^{*}$-homomorphism of a $\mathrm{C}^{*}$-algebra, it is contractive. This implies

$$
\forall x \in \mathfrak{A}: \quad\left\|\psi\left(\sigma_{t}(x)\right)\right\| \leq\|\psi(x)\|
$$

8. Given any $z \in \widetilde{\mathfrak{A}}$, we can now show that $\rho_{t, \xi, \eta}(z)$ is a bounded sesquilinear function of $\xi$ and $\eta$. For boundedness, we will show more precisely that

$$
\left|\rho_{t, \xi, \eta}(z)\right| \leq\|z\|\|\rho\|\|\eta\|
$$

Let $z, \xi, \eta$ be given, and choose $\epsilon>0$. By the Kaplansky density theorem and the normality of $\rho_{t, \xi, \eta}$, there exists $x \in \mathfrak{A}$ such that $\|\psi(x)\| \leq\|z\|$ and $\left|\rho_{t, \xi, \eta}(z-\psi(x))\right|<\epsilon$. Then

$$
\begin{aligned}
\left|\rho_{t, \xi, \eta}(z)\right| & \leq\left|\rho_{t, \xi, \eta}(\psi(x))\right|+\left|\rho_{\xi, \eta}(z-\psi(x))\right| \\
& \leq \epsilon+\left|\left\langle\psi\left(\sigma_{t}(x)\right) \xi, \eta\right\rangle\right| \\
& \leq \epsilon+\left\|\psi\left(\sigma_{t}(x)\right)\right\|\|\xi\|\|\eta\| \\
& \leq \epsilon+\|\psi(x)\|\|\xi\|\|\eta\| \\
& \leq \epsilon+\|z\|\|\xi\|\|\eta\| .
\end{aligned}
$$

Letting $\epsilon \rightarrow 0$, we have 5.8 .

To show linearity in $\xi$, let $c_{1}, c_{2} \in \mathbb{C}$ and $\xi_{1}, \xi_{2}, \eta \in \mathfrak{H}$ be given, and choose $\epsilon>0$. By Kaplansky density and the normality of $\rho_{t, \xi_{1}, \eta}, \rho_{t, \xi_{2}, \eta}$, and $\rho_{t, c_{1} \xi_{1}+c_{2} \xi_{2}, \eta}$, there 
exists $x \in \mathfrak{A}$ such that $\|\psi(x)\| \leq\|z\|$ and the three inequalities

$$
\begin{array}{r}
\left|\rho_{t, c_{1} \xi_{1}+c_{2} \xi_{2}, \eta}(z-\psi(x))\right|<\epsilon \\
\left|c_{1}\right|\left|\rho_{t, \xi_{1}, \eta}(z-\psi(x))\right|<\epsilon \\
\left|c_{2}\right|\left|\rho_{t, \xi_{2}, \eta}(z-\psi(x))\right|<\epsilon
\end{array}
$$

all hold. Then

$$
\begin{aligned}
& \left|\rho_{t, c_{1} \xi_{1}+c_{2} \xi_{2}, \eta}(z)-c_{1} \rho_{t, \xi_{1}, \eta}(z)-c_{2} \rho_{t, \xi_{2}, \eta}(z)\right| \\
\leq & \left|\rho_{t, c_{1} \xi_{1}+c_{2} \xi_{2}, \eta}(z-\psi(x))\right|+\left|c_{1}\right|\left|\rho_{t, \xi_{1}, \eta}(z-\psi(x))\right|+\left|c_{2}\right|\left|\rho_{t, \xi_{2}, \eta}(z-\psi(x))\right| \\
& +\left|\rho_{t, c_{1} \xi_{1}+c_{2} \xi_{2}, \eta}(\psi(x))-c_{1} \rho_{t, \xi_{1}, \eta}(\psi(x))-c_{2} \rho_{t, \xi_{2}, \eta}(\psi(x))\right| \\
\leq & 3 \epsilon+\left|\left\langle\psi\left(\sigma_{t}(x)\right)\left(c_{1} \xi_{1}+c_{2} \xi_{2}\right), \eta\right\rangle-c_{1}\left\langle\psi\left(\sigma_{t}(x)\right) \xi_{1}, \eta\right\rangle-c_{2}\left\langle\psi\left(\sigma_{t}(x)\right) \xi_{2}, \eta\right\rangle\right|=3 \epsilon
\end{aligned}
$$

and as this is true for all $\epsilon>0$, we conclude that

$$
\rho_{t, c_{1} \xi_{1}+c_{2} \xi_{2}, \eta}(z)=c_{1} \rho_{t, \xi_{1}, \eta}(z)+c_{2} \rho_{t, \xi_{2}, \eta}(z)
$$

Conjugate-linearity in $\eta$ is, of course, established in the same way.

9. We therefore obtain an operator in $B(\mathfrak{H})$, which we call $\widetilde{\sigma}_{t}(z)$, characterized by the property

$$
\forall \xi, \eta \in \mathfrak{H}: \quad \rho_{t, \xi, \eta}(z)=\left\langle\widetilde{\sigma}_{t}(z) \xi, \eta\right\rangle
$$

We now have a function (not yet known to be linear, continuous, multiplicative, or self-adjoint) $\widetilde{\sigma}_{t}: \widetilde{\mathfrak{A}} \rightarrow B(\mathfrak{H})$ which extends the unital ${ }^{*}$-endomorphism $S_{t}$ : $\psi(\mathfrak{A}) \rightarrow \psi(\mathfrak{A})$. 
10. The function $\widetilde{\sigma}_{t}$ is contractive, because

$$
\left\|\widetilde{\sigma}_{t}(z)\right\|=\sup _{\xi, \eta \in \mathfrak{H}_{1}}\left|\left\langle\widetilde{\sigma}_{t}(z) \xi, \eta\right\rangle\right|=\sup _{\xi, \eta \in \mathfrak{H}_{1}}\left|\rho_{t, \xi, \eta}(z)\right| \leq \sup _{\xi, \eta \in \mathfrak{H}_{1}}\|z\|\|\xi\|\|\eta\|=\|z\|
$$

by equation (5.8).

11. Weak and strong-* continuity of $\widetilde{\sigma}_{t}$ are straightforward consequence of the normality of the $\rho_{t, \xi, \eta}$. Indeed, if $z_{\nu} \rightarrow z$ weakly in the unit ball $\widetilde{\mathfrak{A}}_{1}$, then for all $\xi, \eta$ it follows that

$$
\left\langle\widetilde{\sigma}_{t}\left(z_{\nu}\right) \xi, \eta\right\rangle=\rho_{t, \xi, \eta}\left(z_{\nu}\right) \rightarrow \rho_{t, \xi, \eta}(z)=\left\langle\widetilde{\sigma}_{t}(z) \xi, \eta\right\rangle
$$

so that $\tilde{\sigma}_{t}\left(z_{\nu}\right) \rightarrow \widetilde{\sigma}_{t}(z)$ in the weak operator topology, which agrees with the weak topology of $\widetilde{\mathfrak{A}}$ on bounded subsets. If $z_{\nu} \rightarrow z$ strong- ${ }^{*}$, then $\left(z_{\nu}-z\right)^{*}\left(z_{\nu}-\right.$ $z) \rightarrow 0$ weakly and $\left(z_{\nu}-z\right)\left(z_{\nu}-z\right)^{*} \rightarrow 0$ weakly, and we repeat the analysis.

12. Since $\widetilde{\sigma}_{t}$ maps the unit ball of $\psi(\mathfrak{A})$ into $\psi(\mathfrak{A})$, it follows from the previous step and the Kaplansky density theorem that it maps the unit ball of $\widetilde{\mathfrak{A}}$ into $\widetilde{\mathfrak{A}}$. Hence $\widetilde{\sigma}_{t}$, initially defined as a map from $\widetilde{\mathfrak{A}}$ into $B(\mathfrak{H})$, is actually a self-map of $\widetilde{\mathfrak{A}}$.

13. Next, we prove that $\widetilde{\sigma}_{t}$ is a ${ }^{*}$-endomorphism of $\widetilde{\mathfrak{A}}$. For multiplicativity, let $z, w \in$ $\widetilde{\mathfrak{A}}$. Using Kaplansky density, choose nets $\left\{x_{\nu}\right\},\left\{y_{\nu}\right\} \subset \mathfrak{A}$ with $\left\|\psi\left(x_{\nu}\right)\right\| \leq\|z\|$ and $\left\|\psi\left(y_{\nu}\right)\right\| \leq\|w\|$ for all $\nu$, and $\psi\left(x_{\nu}\right) \rightarrow z$ and $\psi\left(y_{\nu}\right) \rightarrow w$ strongly. Then $\left\|\psi\left(x_{\nu}\right) \psi\left(y_{\nu}\right)\right\| \leq\|z\|\|w\|$ for all $\nu$ and since multiplication is jointly strongly continuous, we have $\psi\left(x_{\nu}\right) \psi\left(y_{\nu}\right) \rightarrow z w$ strongly. Then since $\widetilde{\sigma}_{t}$ is strongly 
continuous and is multiplicative on $\psi(\mathfrak{A})$,

$$
\begin{aligned}
\widetilde{\sigma}_{t}(z w) & =\widetilde{\sigma}_{t}\left(\lim _{\mu, \nu} \psi\left(x_{\nu}\right) \psi\left(y_{\mu}\right)\right) \\
& =\lim _{\mu, \nu} \widetilde{\sigma}_{t}\left(\psi\left(x_{\nu}\right) \psi\left(y_{\mu}\right)\right) \\
& =\lim _{\mu, \nu} \widetilde{\sigma}_{t}\left(\psi\left(x_{\nu}\right)\right) \widetilde{\sigma}_{t}\left(\psi\left(y_{\mu}\right)\right) \\
& =\widetilde{\sigma}_{t}(z) \widetilde{\sigma}_{t}(w) .
\end{aligned}
$$

For linearity, let $c_{1}, c_{2} \in \mathbb{C}$ and $z, w \in \widetilde{\mathfrak{A}}$ be given. Choose $\left\{x_{\nu}\right\}$ and $\left\{y_{\mu}\right\}$ as before; then for all $\mu, \nu$ we have $\left\|c_{1} x_{\nu}+c_{2} y_{\mu}\right\| \leq\left|c_{1}\right|\|z\|+\left|c_{2}\right|\|w\|$, so that $\left\{c_{1} x_{\nu}+c_{2} y_{\mu}\right\}$ is contained in a bounded subset of $\widetilde{\mathfrak{A}}$. The calculation then proceeds as for multiplicativity. Self-adjointness is proved similarly.

14. Finally, it is clear that $\widetilde{\sigma}_{0}=i d$, and for all $s, t \geq 0$ and all $x \in \mathfrak{A}$,

$$
\widetilde{\sigma}_{s+t}(\psi(x))=\psi\left(\sigma_{s+t}(x)\right)=\psi\left(\sigma_{s}\left(\sigma_{t}(x)\right)\right)=\widetilde{\sigma}_{s}\left(\psi\left(\sigma_{t}(x)\right)\right)=\widetilde{\sigma}_{s}\left(\widetilde{\sigma}_{t}(\psi(x))\right)
$$

so that $\widetilde{\sigma}_{s+t}$ and $\widetilde{\sigma}_{s} \circ \widetilde{\sigma}_{t}$ agree on the ultraweakly dense subset $\psi(\mathfrak{A}) \subset \widetilde{\mathfrak{A}}$; as both are normal, they are equal.

As one corollary, we can now find many dense subspaces of $\mathfrak{H}$. Recall that $\psi(\mathcal{P}) V H$ is dense by the standard properties of the minimal Stinespring dilation plus the fact that $\mathcal{P}$ is norm-dense in $\mathfrak{A}$.

Lemma 5.4.4. For any finite set $F \subset[0, \infty)$ let $\mathcal{P}^{(F)}$ denote those elements of $\mathcal{P}$ which do not use any time indices from $F$. Then for all finite $F \subset[0, \infty), \psi\left(P^{(F)} V H\right.$ is dense in $\mathfrak{H}$. 
Proof. Consider a general vector of the form $\sigma_{t_{1}}\left(i\left(a_{1}\right)\right) \cdots \sigma_{t_{n}}\left(i\left(a_{n}\right)\right) V h$, which we already know to be total in $\mathfrak{H}$. We proceed by induction on $n$. In the case $n=1$ we have for any $\tilde{t}$ that

$$
\left\|\left(\sigma_{t}(i(a))-\sigma_{\tilde{t}}(i(a))\right) V h\right\|^{2}=\mathfrak{S}\left(t ; a^{*} a\right)-\mathfrak{S}\left(\tilde{t}, t ; a^{*}, a\right)-\mathfrak{S}\left(t, \tilde{t} ; a^{*}, a\right)+\mathfrak{S}\left(\tilde{t} ; a^{*} a\right) .
$$

As $\tilde{t} \rightarrow t$, this approaches zero by the continuity properties of $\mathfrak{S}$. Inductively, we can approximate $\sigma_{t_{2}}\left(i\left(a_{2}\right)\right) \cdots \sigma_{t_{n}}\left(i\left(a_{n}\right)\right) V h$ by a vector in $\psi\left(\mathcal{P}^{(F)}\right) V H$, which we then use as our $h$ and proceed as before.

Before establishing our main continuity result, one more preliminary is needed.

Proposition 5.4.5. The Hilbert space $\mathfrak{H}$ is separable.

Proof. Let $H_{0}$ be a countable dense subset of $H$, and $\mathcal{A}_{0}$ a countable ultraweakly dense subset of $\mathcal{A}$. We may assume WLOG that $\mathcal{A}_{0}$ is a self-adjoint $\mathbb{Q}$-subalgebra, so that its unit ball is strongly dense in the unit ball of $\mathcal{A}$ by Kaplansky's theorem.

We will show that the countable set

$$
\left\{\psi\left(\sigma_{t_{1}}\left(i\left(x_{1}\right)\right) \ldots \sigma_{t_{n}}\left(i\left(x_{n}\right)\right)\right) V h \mid 0 \leq t_{1}, \ldots, t_{n} \in \mathbb{Q} ; x_{1}, \ldots, x_{n} \in \mathcal{A}_{0} ; h \in H_{0}\right\}
$$

spans a dense subset of $\mathfrak{H}$. We already know that $\psi(\mathcal{P}) V H$ has dense span, so it suffices to show that vectors in $\psi(\mathcal{P}) V H$ can be norm-approximated by vectors of the prescribed form. Let $\tau_{1}, \ldots, \tau_{n} \geq 0, y_{1}, \ldots, y_{n} \in \mathcal{A}$, and $k \in H$. By the triangle 
inequality, we have for any $h \in H_{0}$, any $t_{1}, \ldots, t_{n} \in \mathbb{Q}_{+}$, and any $x_{1}, \ldots, x_{n} \in \mathcal{A}_{0}$ that

$$
\begin{aligned}
& \left\|\psi\left(\sigma_{\tau_{1}}\left(i\left(y_{1}\right)\right) \ldots \sigma_{\tau_{n}}\left(i\left(y_{n}\right)\right)\right) V k-\psi\left(\sigma_{t_{1}}\left(i\left(x_{1}\right)\right) \ldots \sigma_{t_{n}}\left(i\left(x_{n}\right)\right)\right) V h\right\| \\
& \leq\left\|\psi\left(\sigma_{\tau_{1}}\left(i\left(y_{1}\right)\right) \ldots \sigma_{\tau_{n}}\left(i\left(y_{n}\right)\right)\right)\right\|\|h-k\| \\
& \quad+\left\|\psi\left[\sigma_{\tau_{1}}\left(i\left(y_{1}\right)\right) \cdots \sigma_{\tau_{n}}\left(i\left(y_{n}\right)\right)-\sigma_{\tau_{1}}\left(i\left(x_{1}\right)\right) \cdots \sigma_{\tau_{n}}\left(i\left(x_{n}\right)\right)\right] V h\right\| \\
& \quad+\left\|\psi\left[\sigma_{\tau_{1}}\left(i\left(x_{1}\right)\right) \cdots \sigma_{\tau_{n}}\left(i\left(x_{n}\right)\right)-\sigma_{t_{1}}\left(i\left(x_{1}\right)\right) \cdots \sigma_{t_{n}}\left(i\left(x_{n}\right)\right)\right] V h\right\| .
\end{aligned}
$$

The first term can be made small by choosing $h$ sufficiently close to $k$. For the second, note that each composition $\psi \circ \sigma_{t} \circ i$ is normal, since it equals the composition $\widetilde{\sigma}_{t} \circ \psi \circ i$; hence $\psi\left(\sigma_{t}\left(i\left(\mathcal{A}_{0}\right)\right)\right)$ is weakly dense in $\psi\left(\sigma_{t}(i(\mathcal{A}))\right)$. By Kaplansky's theorem, it follows that the unit ball of $\psi\left(\sigma_{t}\left(i\left(\mathcal{A}_{0}\right)\right)\right)$ is strongly dense in the unit ball of $\psi\left(\sigma_{t}(i(\mathcal{A}))\right)$; this plus the joint strong continuity of multiplication implies that $\left\{\psi\left(\sigma_{t_{1}}\left(i\left(x_{1}\right)\right) \ldots \sigma_{s_{n}}\left(i\left(x_{n}\right)\right)\right) \mid s_{1}, \ldots, s_{n} \geq 0 ; x_{1}, \ldots, x_{n} \in \mathcal{A}_{0}\right\}$ is strongly dense in $\left\{\psi\left(\sigma_{t_{1}}\left(i\left(y_{1}\right)\right) \ldots \sigma_{t_{n}}\left(i\left(y_{n}\right)\right)\right) \mid t_{1}, \ldots, t_{n} \geq 0 ; y_{1}, \ldots, y_{n} \in \mathcal{A}\right\}$. Hence, once $h$ has been fixed, an appropriate choice of $x_{1}, \ldots, x_{n}$ makes the second term arbitrarily small. So far we have shown that vectors of the form

$$
\psi\left(\sigma_{\tau_{1}}\left(i\left(x_{1}\right)\right) \cdots \sigma_{\tau_{n}}\left(i\left(x_{n}\right)\right)\right) V h \quad \tau_{1}, \ldots, \tau_{n} \geq 0 ; x_{1}, \ldots, x_{n} \in \mathcal{A}_{0} ; h \in H_{0}
$$

are total in $\mathfrak{H}$. It remains to prove that such vectors remain total under the added restriction that the $\tau_{i}$ be rational. Let $\xi \in \psi(\mathcal{P}) V H$ be orthogonal to all vectors of the form (5.10). That is, we let $z_{1}, \ldots, z_{m} \in \mathcal{A}, \eta \in H$, and $s_{1}, \ldots, s_{m} \geq 0$ such that, 
for all $x_{1}, \ldots, x_{n} \in \mathcal{A}_{0}$, all $0 \leq t_{1}, \ldots, t_{n} \in \mathbb{Q}$, and all $h \in H_{0}$,

$$
\begin{aligned}
0 & =\left\langle\psi\left(\sigma_{t_{1}}\left(i\left(x_{1}\right)\right) \cdots \sigma_{t_{n}}\left(i\left(x_{n}\right)\right)\right) V h, \psi\left(\sigma_{s_{1}}\left(i\left(z_{1}\right)\right) \cdots \sigma_{s_{m}}\left(i\left(z_{m}\right)\right)\right) V \eta\right\rangle \\
& =\left\langle V^{*} \psi\left(\sigma_{s_{m}}\left(i\left(z_{m}^{*}\right)\right) \ldots \sigma_{s_{1}}\left(i\left(z_{1}^{*}\right)\right) \sigma_{t_{1}}\left(i\left(x_{1}\right)\right) \cdots \sigma_{t_{n}}\left(i\left(x_{n}\right)\right)\right) V h, \eta\right\rangle \\
& =\left\langle\mathfrak{S}\left(\vec{s}^{*} \vee \vec{t} ; \vec{z}^{*} \vee \vec{x}\right) \xi, \eta\right\rangle
\end{aligned}
$$

where we introduce the notation $\left(s_{1}, \ldots, s_{m}\right)^{*}=\left(s_{m}, \ldots, s_{1}\right)$ for $s_{1}, \ldots, s_{m} \geq 0$ and $\left(z_{1}, \ldots, z_{m}\right)^{*}=\left(z_{m}^{*}, \ldots, z_{1}^{*}\right)$ for $z_{1}, \ldots, z_{m} \in \mathcal{A}$. Now for any $\vec{t} \in[0, \infty)^{n}$, let $\left\{\overrightarrow{t_{k}}\right\} \subset$ $\mathbb{Q}_{+}^{n}$ such that $\vec{s}^{*} \vee \vec{t}_{k} \rightarrow \vec{s}^{*} \vee \vec{t}$ non-crossingly; then by Proposition (5.3.3),

$$
\left\langle\mathfrak{S}\left(\vec{s}^{*} \vee \vec{t} ; \vec{z}^{*} \vee \vec{x}\right) \xi, \eta\right\rangle=\lim _{k \rightarrow \infty}\left\langle\mathfrak{S}\left(\vec{s}^{*} \vee \vec{t}_{k} ; \vec{z}^{*} \vee \vec{x}\right) \xi, \eta\right\rangle=0
$$

We thus see that $\xi$ must be orthogonal to a known total set and hence zero.

Theorem 5.4.6. For any $a \in \widetilde{\mathfrak{A}}, t \mapsto \widetilde{\sigma}_{t}(a)$ is ultraweakly continuous for all $t>0$.

Proof. We establish this in a series of steps.

1. For any $a \in \mathcal{A}_{0}$ and $\xi, \eta \in \psi(\mathcal{P}) V H$, the value of $\left\langle\widetilde{\sigma}_{t}(\psi(a)) \xi, \eta\right\rangle=\left\langle\psi\left(\sigma_{t}(a)\right) \xi, \eta\right\rangle$ is given by a certain Sauvageot moment polynomial; explicitly, if $a=\sigma_{\tau_{1}}\left(i\left(x_{1}\right)\right) \cdots \sigma_{\tau_{n}}\left(i\left(x_{n}\right)\right), \xi=\sigma_{s_{1}}\left(i\left(y_{1}\right)\right) \cdots \sigma_{s_{m}}\left(i\left(y_{n}\right)\right) V \xi_{0}$, and $\eta=\sigma_{u_{1}}\left(i\left(z_{1}\right)\right) \cdots \sigma_{u_{\ell}}\left(i\left(z_{\ell}\right)\right) V \eta_{0}$, then

$$
\left\langle\widetilde{\sigma}_{t}(\psi(a)) \xi, \eta\right\rangle=\left\langle\pi\left(\mathfrak{S}\left(\vec{u}^{*} \vee(\vec{\tau}+t) \vee \vec{s} ; \vec{z}^{*} \vee \vec{x} \vee \vec{y}\right)\right) \xi_{0}, \eta_{0}\right\rangle
$$

2. Given $\vec{\tau}$ and a time $t_{0} \geq 0$, let $F$ be the set of times in $\vec{\tau}+t_{0}$. Taking any $\xi_{0}, \eta_{0} \in \psi\left(P^{(F)}\right) V H$, which is dense by lemma (5.4.4), we see by proposition (5.3.3) that the above expression is continuous at $t_{0}$, since if $t \rightarrow t_{0}$ within a 
sufficiently small neighborhood of $t_{0}$ then $\vec{u}^{*} \vee(\vec{\tau}+t) \vee \vec{s} \rightarrow \vec{u}^{*} \vee\left(\vec{\tau}+t_{0}\right) \vee \vec{s}$ non-crossingly. We therefore have that $t \mapsto\left\langle\widetilde{\sigma}_{t}(\psi(a)) \xi, \eta\right\rangle$ is continuous at $t_{0}$ for all $\xi, \eta \in \psi\left(\mathcal{P}^{(F)}\right) V H$ and all $a \in \mathcal{A}_{0}$.

3. By Lemma (5.4.1), this implies that $t \mapsto\left\langle\widetilde{\sigma}_{t}(\psi(a)) \xi, \eta\right\rangle$ is continuous at $t_{0}$ for all $\xi, \eta \in \mathfrak{H}$ and all $a \in \mathcal{A}_{0}$.

4. Now let $a \in \widetilde{\mathfrak{A}}$. By Kaplansky density, there is a sequence $\left\{a_{n}\right\} \subset \mathfrak{A}_{0}$ such that $\psi\left(a_{n}\right) \rightarrow a$ in SOT. We can use a sequence rather than a net because the separability of $\mathfrak{H}$, established in Proposition (5.4.5), implies the SOT-metrizability of $B(\mathfrak{H})$ ([Bla06] III.2.2.27). Then for any $\xi, \eta \in \mathfrak{H}$,

$$
\left\langle\widetilde{\sigma}_{t}(a) \xi, \eta\right\rangle=\lim _{n}\left\langle\widetilde{\sigma}_{t}\left(\psi\left(a_{n}\right)\right) \xi, \eta\right\rangle
$$

so that the left-hand side, as a function of $t$, is a pointwise limit of a sequence of continuous functions, hence measurable. That is, $t \mapsto \widetilde{\sigma}_{t}(a)$ is WOTmeasurable; as the $\widetilde{\sigma}$ are contractions and the WOT agrees with the ultraweak topology on bounded subsets, $t \mapsto \widetilde{\sigma}_{t}(a)$ is ultraweakly measurable at all $t \geq 0$.

5. Since each $\widetilde{\sigma}_{t}$ is normal, there is a corresponding preadjoint semigroup $\left\{\rho_{t}\right\}$ on $\widetilde{\mathfrak{A}}_{*}$ given by $\rho_{t} f=f \circ \widetilde{\sigma}_{t}$, as discussed in section 1.4.1, such that for each $f \in \widetilde{\mathfrak{A}}_{*}$, $t \mapsto \rho_{t}(f)$ is weakly measurable at all $t \geq 0$.

6. Since $\mathfrak{H}$ is separable and $\widetilde{\mathfrak{A}} \subset B(\mathfrak{H})$, it follows that $\widetilde{\mathfrak{A}}_{*}$ is a separable Banach space. By section 1.4.1, the weak measurability of $\left\{\rho_{t}\right\}$ is therefore equivalent to its weak continuity at times $t>0$. This is then equivalent to the ultraweak continuity of $t \mapsto \widetilde{\sigma}_{t}$. 
Theorem 5.4.7. $\left(\widetilde{\mathfrak{A}}, \psi \circ i, \widetilde{\mathbb{E}},\left\{\widetilde{\sigma}_{t}\right\}\right)$ is a strong dilation of $\left(\mathcal{A},\left\{\phi_{t}\right\}\right)$.

Proof. By the definition of $\widetilde{\mathbb{E}}$, equation 5.3 , and theorem 4.4.5,

$$
\begin{aligned}
\widetilde{\mathbb{E}} \circ \widetilde{\sigma}_{t} \circ \psi & =\widetilde{\mathbb{E}} \circ \psi \circ \sigma_{t} \\
& =\mathbb{E} \circ \sigma_{t} \\
& =\phi_{t} \circ \mathbb{E} \\
& =\phi_{t} \circ \widetilde{\mathbb{E}} \circ \psi .
\end{aligned}
$$

Since both $\phi_{t} \circ \widetilde{\mathbb{E}}$ and $\widetilde{\mathbb{E}} \circ \widetilde{\sigma}_{t}$ are normal, and since they are equal on the ultraweakly dense subset $\psi(\mathfrak{A}) \subset \widetilde{\mathfrak{A}}$, they must be equal.

So far, theorem 5.4 .6 leaves open the question whether $\left\{\widetilde{\sigma}_{t}\right\}$ is point-weakly continuous at $t=0$. I do not know when, if ever, that would fail to be the case; however, in case it does, we can remedy the situation by taking a suitable quotient.

Lemma 5.4.8. Let $A$ be a separable $W^{*}$-algebra and $\left\{\alpha_{t}\right\}$ an $e_{0}$-semigroup on $A$ which is point-weakly continuous at all $t>0$. Then $\alpha_{t}$ is point-weakly continuous at 0 iff

$$
\bigcap_{t>0} \operatorname{ker} \alpha_{t}=\{0\} .
$$

Proof. The point-weak continuity of $\alpha_{t}$ at $t=0$ is equivalent to the weak (equivalently, strong) continuity at $t=0$ of the adjoint semigroup $\left\{\rho_{t}\right\}$ on $A_{*}$ defined by $\left(\rho_{t} f\right)=$ $f \circ \alpha_{t}$. As mentioned in section 1.4.1, this is equivalent to the condition

$$
\overline{\bigcup_{t>0} \rho_{t} A_{*}}=A_{*}
$$


since $A_{*}$ is assumed separable. Now the annihilator of the left-hand side is

$$
\begin{aligned}
\bigcup_{t>0} \rho_{t} A_{*}^{\perp} & =\left\{a \in A \mid \forall t>0: \forall f \in A_{*}:\left(\rho_{t} f\right)(a)=0\right\} \\
& =\left\{a \in A \mid \forall t>0: \forall f \in A_{*}: f\left(\alpha_{t}(a)\right)=0\right\} \\
& =\left\{a \in A \mid \forall t>0: \alpha_{t}(a)=0\right\} \\
& =\bigcap_{t>0} \operatorname{ker} \alpha_{t}
\end{aligned}
$$

because $A_{*}$ separates points on $A$.

Theorem 5.4.9. Let $\mathcal{A}$ be a separable $W^{*}$-algebra and $\left\{\phi_{t}\right\}$ a $C P_{0}$-semigroup on $\mathcal{A}$. Then there exists a unital strong dilation of $\left\{\phi_{t}\right\}$ to an $E_{0}$-semigroup on a separable $W^{*}$-algebra.

Proof. The dilation $\left(\widetilde{\mathfrak{A}}, \psi \circ i, \widetilde{\mathbb{E}},\left\{\widetilde{\sigma}_{t}\right\}\right)$ constructed in this chapter satisfies all the requirements except possibly point-ultraweak continuity at $t=0$.

We now let

$$
\mathcal{R}=\bigcap_{t>0} \operatorname{ker} \widetilde{\sigma}_{t}
$$

This is an ultraweakly closed ideal in $\widetilde{\mathfrak{A}}$; we use $\widehat{\mathfrak{A}}$ for the quotient $\widetilde{\mathfrak{A}} / \mathcal{R}$, which is another separable $\mathrm{W}^{*}$-algebra. Because $\widetilde{\sigma}_{t}(\mathcal{R}) \subset \mathcal{R}$ for each $t>0$, we obtain for each $t>0$ a map $\widehat{\sigma}_{t}: \widehat{\mathfrak{A}} \rightarrow \widehat{\mathfrak{A}}$ characterized by the commutative diagram

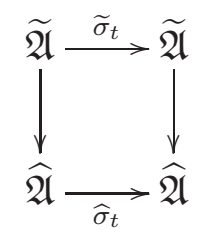

Defining also $\widehat{\sigma}_{0}=\mathrm{id}_{\widehat{\mathscr{A}}}$, we see that $\left\{\widehat{\sigma}_{t}\right\}$ inherits from $\left\{\widetilde{\sigma}_{t}\right\}$ the properties of being an $\mathrm{e}_{0}$-semigroup and of point-ultraweak continuity at $t>0$. Furthermore, 
$\bigcap_{t>0} \operatorname{ker} \widehat{\sigma}_{t}=\{0\}$ by construction, so that $\left\{\widehat{\sigma}_{t}\right\}$ is point-weakly continuous at $t=0$ and hence is an $\mathrm{E}_{0}$-semigroup. Our embedding of $\mathcal{A}$ into $\widehat{\mathfrak{A}}$ is given by $q \circ \psi \circ i$, where $\widetilde{\mathfrak{A}} \stackrel{q}{\rightarrow} \widehat{\mathfrak{A}}$ is the quotient map; this is injective because, if $a \in \mathcal{A}$ is such that $q(\psi(i(a)))=0$, then $\psi(i(a)) \in \mathcal{R}$, so that for all $t>0$ one has

$$
\begin{aligned}
\widetilde{\sigma}_{t}(\psi(i(a))) & =0 \\
\psi\left(\sigma_{t}(i(a))\right) & =0 \\
\sigma_{t}(i(a)) & =0 \\
\mathbb{E}\left[\sigma_{t}(i(a))\right] & =0 \\
\phi_{t}(\mathbb{E}[i(a)]) & =0 \\
\phi_{t}(a) & =0
\end{aligned}
$$

and since $\phi_{t}(a) \rightarrow a$ as $t \rightarrow 0^{+}$this implies $a=0$. To construct our retraction, we first note that $\mathcal{R} \subset$ ker $\widetilde{\mathbb{E}}$; indeed, if $a \in \mathcal{R}$ then for all $t>0$ we have

$$
\begin{aligned}
\widetilde{\sigma}_{t}(a) & =0 \\
\widetilde{\mathbb{E}} \circ \widetilde{\sigma}_{t}(a) & =0 \\
\phi_{t} \circ \widetilde{\mathbb{E}}(a) & =0
\end{aligned}
$$

and by letting $t \rightarrow 0^{+}$we conclude $\widetilde{\mathbb{E}}(a)=0$. Hence, ker $q \subset$ ker $\widetilde{\mathbb{E}}$, so there is a unique map $\widehat{\mathbb{E}}: \widehat{\mathfrak{A}} \rightarrow \mathcal{A}$ with $\widetilde{\mathbb{E}}=\widehat{\mathbb{E}} \circ q$. This map satisfies $\widehat{\mathbb{E}} \circ q \circ \psi \circ i=\widetilde{\mathbb{E}} \circ \psi \circ i=\mathrm{id}_{\mathcal{A}}$, so it is a retraction with respect to the given embedding. Finally,

$$
\widehat{\mathbb{E}} \circ \widehat{\sigma}_{t} \circ q=\widehat{\mathbb{E}} \circ q \circ \widetilde{\sigma}_{t}=\widetilde{\mathbb{E}} \circ \widetilde{\sigma}_{t}=\phi_{t} \circ \widetilde{\mathbb{E}}=\phi_{t} \circ \widehat{\mathbb{E}} \circ q,
$$


and since the image of $q$ generates $\widehat{\mathfrak{A}}$ this implies $\widehat{\mathbb{E}} \circ \widehat{\sigma}_{t}=\phi_{t} \circ \widehat{\mathbb{E}}$. We therefore have a strong dilation of the original semigroup. 


\section{CHAPTER 6}

\section{COVARIANT FILTRATIONS FOR SAUVAGEOT DILATIONS}

\subsection{Introduction}

In the commutative Daniell-Kolmogorov construction, the retraction $\mathbb{E}: \mathfrak{A} \rightarrow$ $\mathcal{A}$ can be interpreted as follows: Given a function $f$ on the path space $\mathscr{S}, \mathbb{E} f$ is the function on the state space $S$ with the property that $(\mathbb{E} f)(x)$ is the best guess at the value of $f(p)$ if the only information we know about path $p$ is that it starts at the point $x$. One can generalize this: For any time $t \geq 0$ one can define a retraction $\mathbb{E}_{t}$ from $\mathfrak{A}$ to the functions on stopped path space $S^{[0, t]}$. The value of $\mathbb{E}_{t} f$ at a stopped path $q$ is the best guess at the value of $f(p)$ if the only information known about path $p$ is that its history up to time $t$ is given by $q$. The conditional expectations $\left\{E_{t}\right\}$ on $\mathfrak{A}$ corresponding to the retractions $\left\{\mathbb{E}_{t}\right\}$ satisfy the filtration property $E_{t} E_{s}=E_{s} E_{t}=E_{s}$ for $s \leq t$, and the fact that the process is Markov implies the covariance property $\sigma_{s} E_{t}=E_{t+s} \sigma_{s}$ for $s, t \geq 0$.

If one is interested in dilations of $\mathrm{cp}_{0}$-semigroups in the context of a theory of noncommutative Markov processes, it may be desirable to construct not only a dilation of the given $\mathrm{cp}_{0}$-semigroup, but also a covariant filtration on the dilation algebra. This was done in the paper [Sau86], in a manner that we now relate. 


\subsection{Strong Right-Liberation}

As we shall see shortly, our filtration will depend upon a method of constructing the Sauvageot product $A \star B$ which takes into account the additional information of a conditional expectation on $A$. In preparation for this we develop a modification of the liberation properties from chapter 2 ,

Definition 6.2.1. Let $C, \nu, \mathfrak{e}, A, B, \rho$ be as in Definition 2.3.1. Let $R: A \rightarrow A$ be a linear map such that $\mathfrak{e} \circ R=\mathfrak{e}$, and let $A_{0} \subset A$ denote the range of $R$. We say that we say that $(A, B, \rho, R)$ is strongly right-liberated if $\mathfrak{e}$ is a left $\left\langle A_{0}, B\right\rangle$ -

module map and for every $n \geq 1, a_{2}, \ldots, a_{n} \in A$, and $b_{1}, \ldots, b_{n} \in B$ satisfying $\nu\left(b_{1}\right)=\cdots=\nu\left(b_{n-1}\right)=0$ and every $x \in A_{0}$,

$$
\mathfrak{e}\left[x b_{1} \stackrel{\circ}{a}_{2} b_{2} \ldots \stackrel{\circ}{a}_{n} b_{n}\right]=0
$$

A strongly right-liberating representation is the corresponding analogue of Definition 2.3.2.

As with right and left liberation, strong right liberation implies an algorithm for calculating $\mathfrak{e}$ on $\langle A, B\rangle$. Accordingly, we introduce three types of words in $\langle A, B\rangle$ : A word of the first type is, as usual, of the form $b_{0} a_{1} b_{1} \ldots a_{\ell} b_{\ell}$ for some $a_{1}, \ldots, a_{\ell} \in A$ and $b_{0}, \ldots b_{\ell} \in B$. A word of the second type is of the form $b_{0} \tilde{a}_{1} b_{1} \stackrel{\circ}{a}_{2} b_{2} \stackrel{\circ}{a}_{3} b_{3} \ldots \stackrel{\circ}{a}_{\ell} b_{\ell}$ where we retain the notation $\stackrel{\circ}{a}=a-\rho(a)$, and introduce the notation $\tilde{a}=a-R(a)$. A word of the third type is of the form $b_{0} R\left(a_{1}\right) b_{1} \stackrel{\circ}{a}_{2} b_{2} \stackrel{\circ}{a}_{3} b_{3} \ldots \stackrel{\circ}{a}_{\ell} b_{\ell}$. As previously, words of all types are said to be in standard form if $\nu\left(b_{1}\right)=\cdots=\nu\left(b_{\ell-1}\right)=0$.

The relevant center-expand-simplify strategy for calculating $\mathfrak{e}$ on words of the 
first type is as follows:

- Center the $b_{i}$ for $0<i<\ell$, expand, and collapse. The result is a sum of standard-form words of the first type, each with length at most $\ell$.

- Center $a_{1}$ with respect to $R$ (that is, write $\left.a_{1}=\tilde{a}_{1}+R\left(a_{1}\right)\right)$ and the $a_{i}$ for $i>1$ with respect to $\rho$. Expand and collapse. The result is a sum of words of the second and third types, with length at most $\ell$, and with length equal to $\ell$ only when in standard form.

- For words not in standard form, un-center the $a_{i}$, expand, and simplify, thereby obtaining a sum of words of the first type with length strictly less than $\ell$. The procedure can then be recursively applied to these.

- On standard-form words of the second type, $\mathfrak{e}$ vanishes by the hypothesis of strong right liberation.

- On standard-form words of the third type, e can be calculated using the left $\left\langle A_{0}, B\right\rangle$-module property:

$$
\mathfrak{e}\left[b_{0} R\left(a_{1}\right) w\right]=b_{0} R\left(a_{1}\right) \mathfrak{e}[w]
$$

where $w$ is a strictly shorter word, on which $\mathfrak{e}$ can be calculated recursively.

We now formalize this strategy into a recursive definition.

- We modify our definitions of right collapse and un-collapse to account for the fact that $a_{1}$ is now centered by $R$ rather than $\rho$. Given $\ell \geq 1, \vec{x} \in \mathcal{W}_{\ell}$, and 
a subset $\vec{\iota} \subset[\ell-1]$, write $\vec{\iota}=\left(i_{1}, \ldots, i_{m}\right)$. For $k=1, \ldots, m$ we define $\beta_{k}=$

$b_{i_{k}} \prod_{j=i_{k}+1}^{i_{k+1}-1} \rho\left(a_{j}\right) b_{j}$ as before. However, $\beta_{0}$ is now always equal to $b_{0}$. In the case $1 \in$ $\vec{\iota}$ we define the modified right collapse $\operatorname{RC}_{s}(\vec{x} ; \vec{\iota})=\left(\beta_{0}, a_{1}, \beta_{1}, \ldots, a_{i_{m}}, \beta_{m}\right)$ as before, but in the case $1 \notin \vec{\imath}$ we define $\operatorname{RC}_{s}(\vec{x} ; \vec{\iota})=\left(\beta_{0}, R\left(a_{1}\right), \beta_{1}, a_{i_{1}}, \ldots, a_{i_{m}}, \beta_{m}\right)$. Similar remarks apply to the modified un-collapse $\mathrm{UC}_{s}(\vec{x} ; \vec{\iota})$.

- We also define, for $\ell>0$ and $\vec{x} \in \mathcal{W}_{\ell}$, the tail $\vec{x}_{+}$to be the tuple obtained by truncating the first two entries. That is, $\left(b_{0}, a_{1}, b_{1} \ldots, a_{\ell}, b_{\ell}\right)_{+}=$ $\left(b_{1}, a_{2}, b_{2}, \ldots, a_{\ell}, b_{\ell}\right)$

- We now recursively define

$$
\begin{aligned}
\operatorname{LM}_{s}\left(b_{0}\right) & =\mathrm{RM}_{s}\left(b_{0}\right)=\mathrm{UM}_{s}\left(b_{0}\right)=b_{0} \\
\operatorname{LM}_{s}(\vec{x}) & =\sum_{\vec{\iota} \subseteq[\ell]} \operatorname{RM}_{s}\left(\mathrm{LC}_{s}(\vec{x} ; \vec{\iota})\right) \prod_{j \in[\ell+1] \backslash \vec{\iota}} \nu\left(x_{2 j+1}\right) \\
\operatorname{RM}_{s}(\vec{x}) & =\sum_{1 \in \vec{\iota} \subsetneq[\ell+1]} \mathrm{UM}_{s}\left(\mathrm{RC}_{s}(\vec{x} ; \vec{\iota})\right)+\sum_{1 \notin \vec{\iota} \subset[\ell+1]} x_{1} R\left(x_{2}\right) \mathrm{UM}_{s}(\vec{x}+\vec{i}-1) \\
\mathrm{UM}_{s}(\vec{x}) & =\sum_{\vec{\iota} \subseteq[\ell+1]} \operatorname{LM}_{s}\left(\mathrm{UC}_{s}(\vec{x} ; \vec{\iota})\right)
\end{aligned}
$$

for $\vec{x} \in \mathcal{W}_{\ell+1}$.

We then arrive at the following theorems, which we state without proof.

Theorem 6.2.2. Let $(A, B, \rho, R)$ be strongly right-liberated in $\mathcal{A}$ with respect to $\mathfrak{e}, \nu$. Then for any $\vec{x} \in \mathcal{W}_{I}$,

$$
\mathfrak{e}[\Pi(\vec{x})]=\operatorname{LM}_{s}(\vec{x}) \mathfrak{e}[\mathbf{1}]
$$


Corollary 6.2.3. Let $(A, B, \rho, R)$ be strongly right-liberated in $\mathcal{A}$ with respect to $\mathfrak{e}, \nu$. Then

$$
\mathfrak{e}[\langle A, B\rangle]=\mathfrak{e}\left[\left\langle A_{0}, B\right\rangle\right]
$$

Corollary 6.2.4. Let $\mathcal{A}$ be a unital algebra, $A, B \subset \mathcal{A}$ unital subalgebras, $\rho: A \rightarrow B$ a unital linear map, $R$ a linear transformation on $A, \nu: \mathcal{A} \rightarrow \mathbb{C}$ a unital linear functional. Suppose $\mathfrak{e}_{1}, \mathfrak{e}_{2}: \mathcal{A} \rightarrow \mathcal{A}$ are linear maps satisfying

- $\mathfrak{e}_{i} \circ \rho=\mathfrak{e}_{i}$

- $\mathfrak{e}_{i} \circ R=\mathfrak{e}_{i}$

- $(A, B, \rho, R)$ is strongly right-liberated with respect to $\mathfrak{e}_{i}, \nu$

for $i=1,2$.

$$
\text { If } \mathfrak{e}_{1}[\mathbf{1}]=\mathfrak{e}_{2}[\mathbf{1}] \text {, then } \mathfrak{e}_{1}=\mathfrak{e}_{2} \text { on }\langle A, B\rangle \text {. }
$$

\subsection{Lifting a Retraction to the Sauvageot Product}

We begin this section with a lemma which is of independent interest.

Proposition 6.3.1. Let $A_{0}$ and $A$ be unital $C^{*}$-algebras, $\iota: A_{0} \rightarrow A$ a unital embedding, and $\epsilon: A \rightarrow A_{0}$ a unital retraction with respect to $\iota$. Let $\pi: A_{0} \rightarrow B(H)$ be a representation. Then the minimal Stinespring triple $(K, \psi, V)$ for the map $\pi \circ \epsilon: A \rightarrow B(H)$ has the property that the image of $V$ is invariant under $\psi \circ \iota\left(A_{0}\right)$; that is, $V V^{*} \psi(\iota(\cdot)) V=\psi(\iota(\cdot)) V$. 
Proof. For any $a_{0} \in A_{0}$ and $h \in H$,

$$
\begin{aligned}
\left\|\psi\left(\iota\left(a_{0}\right)\right) V h-V V^{*} \psi\left(\iota\left(a_{0}\right)\right) V h\right\|^{2}= & \left\langle\psi\left(\iota\left(a_{0}\right)\right) V h, \psi\left(\iota\left(a_{0}\right)\right) V h\right\rangle \\
& -2 \operatorname{Re}\left\langle\psi\left(\iota\left(a_{0}\right)\right) V h, V V^{*} \psi\left(\iota\left(a_{0}\right)\right) V h\right\rangle \\
& +\left\langle V V^{*} \psi\left(\iota\left(a_{0}\right)\right) V h, V V^{*} \psi\left(\iota\left(a_{0}\right)\right) V h\right\rangle \\
= & \left\langle V^{*} \psi\left(\iota\left(a_{0}^{*} a_{0}\right)\right) V h, h\right\rangle-\left\langle V^{*} \psi\left(\iota\left(a_{0}\right)\right) V h, V^{*} \psi\left(\iota\left(a_{0}\right)\right) V h\right\rangle \\
= & \left\langle\pi\left(\epsilon\left(\iota\left(a_{0}^{*} a_{0}\right)\right)\right) h, h\right\rangle-\left\langle\pi\left(\epsilon\left(\iota\left(a_{0}\right)\right)\right) h, \pi\left(\epsilon\left(\iota\left(a_{0}\right)\right)\right) h\right\rangle \\
= & \left\langle\pi\left(a_{0}^{*} a_{0}\right) h, h\right\rangle-\left\langle\pi\left(a_{0}\right) h, \pi\left(a_{0}\right) h\right\rangle=0 .
\end{aligned}
$$

We now apply this in the context of lifting a Sauvageot retraction.

Theorem 6.3.2. Let $(A, B, \phi, \omega)$ be a $C P C^{*}$-tuple (resp. $C P W^{*}$-tuple), $A_{0}$ another unital $C^{*}$-algebra (resp. $W^{*}$-algebra), $A_{0} \stackrel{\iota}{\rightarrow} A$ a (normal) unital embedding, $A \stackrel{\epsilon}{\rightarrow} A_{0}$ a corresponding (normal) retraction such that $\phi \circ \iota \circ \epsilon=\phi$. Forming the Sauvageot products of the CP-tuples $(A, B, \phi, \omega)$ and $\left(A_{0}, B, \phi \circ \iota, \omega\right)$ with Sauvageot retractions $\theta$ and $\theta_{0}$ respectively, and letting $\iota^{*}$ denote the map $\iota \star i d_{B}: A_{0} \star B \rightarrow A \star B$, there exists a unique retraction $\epsilon^{*}: A \star B \rightarrow A_{0} \star B$ such that the diagrams
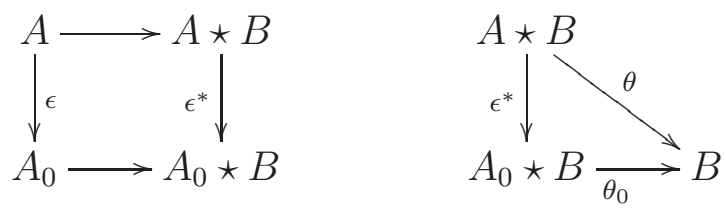

commute, and such that $\left(A \star B, \psi_{L}, \psi_{R}, \theta \circ \psi_{R}, \varpi, \epsilon^{*} \circ \iota^{*}\right)$ is a strongly right-liberating representation of $(A, B, \phi, \epsilon \circ \iota)$. 
Proof. First we show that there exists a faithful representation $\left(H, \Omega, \pi_{R}, K, V, \pi_{L}\right)$ of $(A, B, \phi, \omega)$ and an $\pi_{L}\left(\iota\left(A_{0}\right)\right)$-invariant subspace $V H \subset K_{0} \subset K$ with the property that $\left(H, \Omega, \pi_{R}, K_{0}, V, \pi_{L} \circ \iota\right)$ is a faithful representation of $\left(A_{0}, B, \phi \circ \iota, \omega\right)$. For this, we begin with a faithful representation $\left(H, \Omega, \pi_{R}\right)$ of $(B, \omega)$. Then, applying Stinespring's theorem to the completely positive map $\pi_{R} \circ \phi \circ \iota: A_{0} \rightarrow B(H)$, we obtain a triple $\left(K_{0}, V, \pi_{L}^{(0)}\right)$ such that $V^{*} \pi_{L}^{(0)}\left(a_{0}\right) V=\pi_{R}\left(\phi\left(\iota\left(a_{0}\right)\right)\right)$. We may assume WLOG that $\left(H, \Omega, \pi_{R}, K_{0}, V, \pi_{L}^{(0)}\right)$ is faithfully decomposable, as otherwise we take its direct sum with some faithful representation of $A_{0}$. Applying Stinespring again to the completely positive map $\pi_{L}^{(0)} \circ \epsilon: A \rightarrow B\left(K_{0}\right)$, we obtain another triple $\left(K, W, \pi_{L}\right)$ such that $W^{*} \pi_{L}(a) W=\pi_{L}^{(0)}(\epsilon(a))$. For simplicity, we suppress the notation and regard $K_{0}$ as a subspace of $K$; by Proposition 6.3.1, $\left(K_{0}, \pi_{L}^{(0)}\right)$ is a subrepresentation of $\left(K, \pi_{L} \circ\right.$ ८). Again we assume without loss of generality that $\left(H, \Omega, \pi_{R}, K, V, \pi_{L}\right)$ is faithfully decomposable, as otherwise we replace $\left(K, \pi_{L}\right)$ by its direct sum with some faithful representation of $A$.

Having completed this task, we now let $A \star B$ be the Sauvageot product realized by this representation on the Hilbert space $\mathfrak{H}=H^{-} \star L$; by 3.4 .11 , the $\mathrm{C}^{*}$-subalgebra (resp. von Neumann subalgebra) generated by $A_{0}$ and $B$ acting on $\mathfrak{H}$ is $A_{0} \star B$. Letting $\mathfrak{H}_{0}=H^{-} \star L_{0}$, which by Remark 3.2 .8 may be regarded as a subspace of $\mathfrak{H}$, we see from Proposition 3.2 .9 that $\mathfrak{H}_{0}$ is $A_{0} \star B$-invariant. It follows that this and the faithfulness of $\left(H, \Omega, \pi_{R}, K_{0}, V, \pi_{L}^{(0)}\right)$ that, letting $C: B(\mathfrak{H}) \rightarrow B\left(\mathfrak{H}_{0}\right)$ be the natural compression, then the image $C\left(A_{0} \star B\right)$ is also isomorphic to $A_{0} \star B$, and (modulo this isomorphism) $C$ restricts to the identity map on $A_{0} \star B$. Then the restriction of 
$C$ to $A \star B$, which we denote $\epsilon^{*}$, is a retraction satisfying the commuting diagrams in the statement of the theorem. It remains to check strong right-liberation. Beginning with a vector in any summand $L_{0}^{+\otimes n} \otimes L_{0}$ or $H \otimes L_{0}^{+\otimes n} \otimes L_{0}$ and applying a word of the form

$$
\left[\psi_{L}\left(a_{1}\right)-\psi_{R}\left(\phi\left(a_{1}\right)\right)\right] \psi_{R}\left(b_{1}\right) \ldots\left[\psi_{L}\left(a_{n}\right)-\psi_{R}\left(\phi\left(a_{n}\right)\right)\right] \psi_{R}\left(b_{n}\right)
$$

produces a vector in a sum of subspaces of the same form. But then applying an element $\psi_{R}(a)-\psi_{R}(\epsilon(\iota(a)))$ yields a sum of vectors in subspaces $\left(L \ominus L_{0}\right) \otimes L^{+\otimes n} \otimes L$, so that projection onto $\mathfrak{H}_{0} \subset \mathfrak{H}$ again returns zero.

The next two results are more easily stated in terms of conditional expectations, but may be translated into the language of retractions if desired.

Proposition 6.3.3. Let $(A, B, \phi, \omega)$ be a CP-tuple, E a conditional expectation on $A$, $A_{1} \subset A$ a $C^{*}$-subalgebra (resp. $W^{*}$-subalgebra) such that $E\left(A_{1}\right) \subset A_{1}$. Then viewing $A_{1} \star B$ as a subalgebra of $A \star B$, we have $E^{*}\left(A_{1} \star B\right) \subset A_{1} \star B$.

Proof. This follows from the uniqueness of Sauvageot products and of the lifted retractions provided by Theorem 6.3 .2 , because the restriction of $E$ is a conditional expectation on $A_{1}$.

Corollary 6.3.4. Let $(A, B, \phi, \omega)$ be a CP-tuple and $E_{1}, E_{2}$ conditional expectations on $A$ such that $E_{1} \circ E_{2}=E_{1}$ (resp. $\left.E_{1} \circ E_{2}=E_{2}\right)$. Then $E_{1}^{*} \circ E_{2}^{*}=E_{1}^{*}$ (resp. $\left.E_{1}^{*} \circ E_{2}^{*}=E_{2}^{*}\right)$

Proof. This follows from the uniqueness of $E_{1}^{*}\left(\right.$ resp. $\left.E_{2}^{*}\right)$ in Theorem 6.3.2, as $E_{1}^{*} \circ E_{2}^{*}$ satisfies the same properties. 


\subsection{The Inductive System: Retractions Onto Initial Segments}

In the construction of our inductive system in chapter 4, we produce retractions $\epsilon_{\gamma}: \mathcal{A}_{\gamma} \rightarrow \mathcal{A}$ for each $\gamma \in \mathcal{F}$. We have now the tools to produce a consistent family of retractions $\epsilon_{\beta, \gamma}: \mathcal{A}_{\gamma} \rightarrow \mathcal{A}_{\beta}$ whenever $\beta \leq \gamma$ is an initial segment.

Definition 6.4.1. Let $\gamma=\left\{t_{1}, \ldots, t_{n}\right\} \in \mathcal{F}$ and $\beta=\left\{t_{1}, \ldots, t_{m}\right\}$ for some $1 \leq m \leq$ $n$. We define a retraction $\epsilon_{\beta, \gamma}: \mathcal{A}_{\gamma} \rightarrow \mathcal{A}_{\beta}$ as follows:

- We proceed by reverse induction to define retractions $\epsilon_{\beta(\ell), \gamma(\ell)}$ for $\ell=m, \ldots, 1$.

- In the base case $\ell=m$ we define $\epsilon_{\beta(m), \gamma(m)}$ to be $\epsilon_{\gamma(m)}$ as defined in chapter 4 .

- Inductively, we define

$$
\epsilon_{\beta(\ell), \gamma(\ell)}=\epsilon_{\beta(\ell+1), \gamma(\ell+1)}^{*}
$$

in the sense of Theorem 6.3.2.

\section{Proposition 6.4.2.}

1. Each map $\epsilon_{\beta, \gamma}$ is a retraction with respect to $f_{\gamma, \beta}$.

2. If $\beta \leq \gamma \leq \delta$ are initial segments, then $\epsilon_{\beta, \gamma} \circ \epsilon_{\gamma, \delta}=\epsilon_{\beta, \delta}$.

Proof.

1. Given $\beta, \gamma$ as in Definition 6.4.1, we inductively prove that $\epsilon_{\beta(\ell), \beta(\ell)} \circ f_{\gamma(\ell), \beta(\ell)}=\mathrm{id}$. The base case $\epsilon_{\gamma} \circ \iota_{\gamma}$ was established in Chapter 4, while the inductive step is just the property $\epsilon^{*} \circ \iota^{*}=$ id from Theorem 6.3.2. 
2. Suppose $\beta=\left\{t_{1}, \ldots, t_{m}\right\}, \gamma=\left\{t_{1}, \ldots, t_{m+n}\right\}$, and $\delta=\left\{t_{1}, \ldots, t_{m+n+k}\right\}$. We first prove inductively that $\epsilon_{\gamma(\ell)} \circ \epsilon_{\gamma(\ell), \delta(\ell)}=\epsilon_{\delta(\ell)}$ for $\ell=m+n, \ldots, 1$. The base case $\ell=m+n$ is trivial, since $\gamma(\ell)$ is a singleton and hence $\epsilon_{\gamma(\ell), \delta(\ell)}=\epsilon_{\delta(\ell)}$ and $\epsilon_{\gamma(\ell)}=\mathrm{id}_{\mathcal{A}}$. Inductively, recall that $\mathcal{A}_{\gamma(\ell)}$ is the Sauvageot product $\mathcal{A}_{\gamma(\ell+1)} \star \mathcal{A}$ with respect to the map $\phi_{t_{\ell+1}-t_{\ell}} \circ \epsilon_{\gamma(\ell+1)}$, and similarly $\mathcal{A}_{\delta(\ell)}=\mathcal{A}_{\delta(\ell+1)} \star \mathcal{A}_{\delta(\ell)}$ with respect to $\phi_{t_{\ell+1}-t_{\ell}} \circ \epsilon_{\delta(\ell)}$. We also have $\epsilon_{\delta(\ell+1)} \circ f_{\delta(\ell+1), \gamma(\ell+1)}=\epsilon_{\gamma(\ell+1)}$ by Proposition 4.4.2, It follows that

$$
\begin{aligned}
\phi_{t_{\ell+1}-t_{\ell}} \circ \epsilon_{\delta(\ell+1)} \circ f_{\delta(\ell+1), \gamma(\ell+1)} \circ \epsilon_{\gamma(\ell+1), \delta(\ell+1)} & =\phi_{t_{\ell+1}-t_{\ell}} \circ \epsilon_{\gamma(\ell+1)} \circ \epsilon_{\gamma(\ell+1), \delta(\ell+1)} \\
& =\phi_{t_{\ell+1}-t_{\ell}} \circ \epsilon_{\delta(\ell+1)}
\end{aligned}
$$

and an application of Corollary 3.4.12 implies that $\epsilon_{\gamma(\ell)} \circ \epsilon_{\gamma(\ell), \delta(\ell)}=\epsilon_{\delta(\ell)}$.

As a particular case we obtain $\epsilon_{\gamma(m)} \circ \epsilon_{\gamma(m), \delta(m)}=\epsilon_{\delta(m)}$, which then becomes the base case of a new induction, proving that $\epsilon_{\beta(\ell), \gamma(\ell)} \circ \epsilon_{\gamma(\ell), \delta(\ell)}=\epsilon_{\beta(\ell), \delta(\ell)}$ for $\ell=m, \ldots, 1$. In the case $\ell=m$ we have $\beta(\ell)$ a singleton, so that $\epsilon_{\beta(\ell), \gamma(\ell)}=\epsilon_{\gamma(\ell)}$ and we reduce to the result just established. The induction on $\ell$ follows by Corollary 6.3.4.

Fix now any $\tau \geq 0$. Let $\mathcal{F}_{\tau} \subset \mathcal{F}$ denote the finite subsets of $[0, \tau]$, and for $\gamma \in \mathcal{F}$ let $\gamma\langle\tau\rangle$ denote $\gamma \cap[0, \tau]$. Now $\left\{\mathcal{A}_{\gamma} \mid \gamma \in \mathcal{F}_{\tau}\right\}$ with the same embeddings $f_{\gamma, \beta}$ from before is an inductive system; let $\mathfrak{A}_{\tau}$ denote its limit, with embeddings $g_{\infty, \gamma}: \mathcal{A}_{\gamma} \rightarrow \mathfrak{A}_{\tau}$. (We note that we can equivalently obtain $\mathfrak{A}_{\tau}$ as the limit of a system indexed by $\mathcal{F}$, with the object $\mathcal{A}_{\gamma\langle\tau\rangle}$ corresponding to the set $\gamma$ and the morphism 
$f_{\gamma\langle\tau\rangle, \beta\langle\tau\rangle}$ corresponding to the inclusion $\beta \leq \gamma$.) Through the universal property of the limit we obtain an embedding $h_{\tau}: \mathfrak{A}_{\tau} \rightarrow \mathfrak{A}$ characterized by

$$
f_{\infty, \gamma}=h_{\tau} \circ g_{\infty, \gamma} \quad \text { for all } \gamma \in \mathcal{F}_{\tau}
$$

Now a slight adaptation of the proof of Proposition 4.4.2 shows that for any inclusion $\beta \leq \gamma$ in $\mathcal{F}$ and any $\ell$ such that $t_{\ell} \in \beta$, one has

$$
\epsilon_{\gamma(\ell), \gamma} \circ f_{\gamma, \beta}=f_{\gamma(\ell), \beta(\ell)} \circ \epsilon_{\beta(\ell), \beta}
$$

It follows that if $\beta \leq \gamma$ are sets both of which contain $\tau$, then

$$
\epsilon_{\gamma\langle\tau\rangle, \gamma} \circ f_{\gamma, \beta}=f_{\gamma\langle\tau\rangle, \beta\langle\tau\rangle} \circ \epsilon_{\beta\langle\tau\rangle, \beta}
$$

Holding $\beta$ fixed and taking a limit in $\gamma$, this implies the existence of a retraction $\mathbb{E}_{\tau}: \mathfrak{A} \rightarrow \mathfrak{A}_{\tau}$ characterized by

$$
\mathbb{E}_{\tau} \circ f_{\infty, \beta}=g_{\infty, \beta\langle\tau\rangle} \circ \epsilon_{\beta\langle\tau\rangle, \beta} \quad \text { for all } \beta \in \mathcal{F}
$$

We let $E_{\tau}=h_{\tau} \circ \mathbb{E}_{\tau}$ denote the corresponding conditional expectation on $\mathfrak{A}$, which is characterized by the property

$$
E_{\tau} \circ f_{\infty, \beta}=f_{\infty, \beta\langle\tau\rangle} \circ \epsilon_{\beta\langle\tau\rangle, \beta} \quad \text { for all } \beta \in \mathcal{F} \text {. }
$$


Proposition 6.4.3 (Filtration). For all $s \leq t, E_{s} E_{t}=E_{s}=E_{t} E_{s}$.

Proof. Since $s \leq t$, we have $\beta\langle s\rangle\langle t\rangle=\beta\langle s\rangle=\beta\langle t\rangle\langle s\rangle$ for all $\beta \in \mathcal{F}$. Then

$$
\begin{aligned}
E_{s} \circ E_{t} \circ f_{\infty, \beta} & =E_{s} \circ f_{\infty, \beta\langle t\rangle} \circ \epsilon_{\beta\langle t\rangle, \beta} \\
& =f_{\infty, \beta\langle s\rangle} \circ \epsilon_{\beta\langle s\rangle, \beta\langle t\rangle} \circ \epsilon_{\beta\langle t\rangle, \beta} \\
& =f_{\infty, \beta\langle s\rangle} \circ \epsilon_{\beta\langle s\rangle, \beta} \\
& =E_{s} \circ f_{\infty, \beta}
\end{aligned}
$$

and

$$
\begin{aligned}
E_{t} \circ E_{s} \circ f_{\infty, \beta} & =E_{t} \circ f_{\infty, \beta\langle s\rangle} \circ \epsilon_{\beta\langle s\rangle, \beta} \\
& =f_{\infty, \beta\langle s\rangle} \circ \epsilon_{\beta\langle s\rangle, \beta} \\
& =E_{s} \circ f_{\infty, \beta}
\end{aligned}
$$

and as the images of the $f_{\infty, \beta}$ generate $\mathfrak{A}$, this implies $E_{t} \circ E_{s}=E_{s}=E_{s} \circ E_{t}$.

Proposition 6.4.4 (Covariance). For any $s, t \geq 0$,

$$
\sigma_{s} E_{t}=E_{t+s} \sigma_{s}
$$

Proof. First, we note that for any initial segment $\beta \leq \gamma$ in $\mathcal{F}$ and any $s \geq 0, \epsilon_{\beta+s, \gamma+s}=$ $\epsilon_{\beta, \gamma}$, since the definition of $\epsilon_{\beta, \gamma}$ depends only on the time differences in $\gamma$. We also 
have $\gamma\langle t\rangle+s=(\gamma+s)\langle t+s\rangle$ for any $s, t \geq 0$ and $\gamma \in \mathcal{F}$. Then

$$
\begin{aligned}
E_{t+s} \circ \sigma_{s} \circ f_{\infty, \gamma} & =E_{t+s} \circ f_{\infty, \gamma+s} \\
& =f_{\infty,(\gamma+s)\langle t+s\rangle} \circ \epsilon_{(\gamma+s)\langle t+s\rangle, \gamma+s} \\
& =f_{\infty, \gamma\langle t\rangle+s} \circ \epsilon_{\gamma\langle t\rangle+s, \gamma+s} \\
& =f_{\infty, \gamma\langle t\rangle+s} \circ \epsilon_{\gamma\langle t\rangle, \gamma} \\
& =\sigma_{s} \circ f_{\infty, \gamma\langle t\rangle} \circ \epsilon_{\gamma\langle t\rangle, \gamma} \\
& =\sigma_{s} \circ E_{t} \circ f_{\infty, \gamma}
\end{aligned}
$$

which implies the result.

\subsection{Covariant Filtrations on $\mathrm{W}^{*}$-Dilations?}

So far we have produced a filtration of conditional expectations $\left\{E_{t}\right\}_{t \geq 0}$ on the $\mathbf{C}^{*}$-dilation algebra $\mathfrak{A}$, which is covariant with respect to the semigroup $\left\{\sigma_{t}\right\}$. In chapter 5, we showed how, when our initial semigroup acts on a $\mathrm{W}^{*}$-algebra, we can modify the dilation to achieve a $W^{*}$-algebra $\widetilde{\mathfrak{A}}$ and a continuous semigroup of normal endomorphisms $\left\{\tilde{\sigma}_{t}\right\}$ of $\widetilde{\mathfrak{A}}$. It is natural, then, to seek a filtration $\left\{\widetilde{E}_{t}\right\}$ of normal conditional expectations on $\widetilde{\mathfrak{A}}$ which is covariant with respect to $\left\{\tilde{\sigma}_{t}\right\}$, which is continuous in the sense that $t \mapsto E_{t}(a)$ is strongly continuous for fixed $a \in \widetilde{\mathfrak{A}}$, and which is also related to our $\mathrm{C}^{*}$-filtration by the diagram

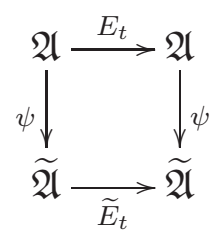


This is very similar in spirit to the question of how to define the maps $\tilde{\sigma}_{t}$, which was addressed in Theorem 5.4.3, A strategy for answering it through similar methods would be as follows:

- For each $\tau \geq 0$, define " $\tau$-moment polynomials" $\mathfrak{S}_{\tau}(\vec{t} ; \vec{a})$ by modifying the recursion in Definition 5.2.2, The point of this would be to show that

$$
\mathfrak{S}_{\tau}(\vec{t} ; \vec{a})=\mathbb{E}_{\tau}\left[\sigma_{t_{1}}\left(i\left(a_{1}\right)\right) \cdots \sigma_{t_{n}}\left(i\left(a_{n}\right)\right)\right]
$$

These $\tau$-moment polynomials should also be weakly continuous in each entry of $\vec{a}$, and jointly strongly continuous in $\vec{t}$ and $\vec{a}$ subject to the non-crossing restriction (indeed, this restriction may only be needed among those times greater than or equal to $\tau$ ) and possibly with an additional restriction that times not cross $\tau$. Finally, for any fixed $\vec{t}$ and $\vec{a}, \mathfrak{S}_{\tau}(\vec{t} ; \vec{a})$ should be strongly continuous in $\tau$ for those $\tau$ not equal to any entry of $\vec{t}$.

- In the spirit of Lemma 5.4.2, one could find for each $y, z \in \mathcal{P}$ and $t \geq 0$ elements $y_{0}, z_{0} \in \mathcal{P}$ and a normal linear map $Q$ on $\mathcal{A}$ such that $\mathbb{E}\left[y E_{t}(x) z\right]=Q \circ \mathbb{E}\left[y_{0} x z_{0}\right]$ for all $x \in \mathfrak{A}$.

- As in Theorem 5.4.3, one would have for all $x \in \mathfrak{A}, y_{0}, z_{0} \in \mathcal{P}$, and $\xi^{\prime}, \eta^{\prime} \in H$ that

$$
\left\langle\psi\left(E_{t}(x)\right) \psi(y) V \xi^{\prime}, \psi(z) V \eta^{\prime}\right\rangle=\left\langle Q\left(\mathbb{E}\left[z_{0}^{*} \psi(x) y_{0}\right]\right) \xi^{\prime}, \eta^{\prime}\right\rangle
$$

and could therefore define $\widetilde{E}_{t}$ by its sesquilinear form

$$
\left\langle\widetilde{E}_{t}(X) \psi(y) V \xi^{\prime}, \psi(z) V \eta^{\prime}\right\rangle=\left\langle Q\left(\widetilde{\mathbb{E}}\left[z_{0}^{*} X y_{0}\right]\right) \xi^{\prime}, \eta^{\prime}\right.
$$


At the time of thesis submission (March 28, 2013), I have not verified the success of this approach. 


\section{CHAPTER 7 \\ PRODUCT SYSTEMS}

\section{$7.1 \quad$ Introduction}

For a Hilbert space $H$, the Fock space

$$
\mathcal{F}(H)=\bigoplus_{n=0}^{\infty} H^{\otimes n}
$$

can be understood as follows: The collection $\left\{H^{\otimes n}\right\}$ forms a bundle of Hilbert spaces over $\mathbb{N}$, and furthermore, a bundle which tensors associatively: there is a family of unitary equivalences $H^{\otimes n} \otimes H^{\otimes m} \rightarrow H^{\otimes(m+n)}$ which compose in such a way that

$H^{\otimes n} \otimes H^{\otimes m} \otimes H^{\otimes k} \rightarrow H^{\otimes(m+n+k)}$ is unambiguous. The Fock space then consists of the square summable sections of this bundle.

When formulated in this way, one can naturally define a "continuous analogue of Fock space" ([Arv89a]-[Arv90b]), called a product system of Hilbert spaces, to be a bundle of Hilbert spaces over $(0, \infty)$ which tensors associatively. As usual when one forms bundles of Hilbert spaces, measurability hypotheses come into play, of which we omit the details here. (The analogue of Fock space is not the bundle itself, but rather the associated Hilbert space of its square-integrable sections.) Without stating all the results precisely, here are some of the relevant aspects of the theory.

1. Product systems of Hilbert spaces are naturally associated with $\mathrm{E}_{0}$-semigroups; indeed, once appropriate morphisms have been defined, there is an equivalence of categories between (equivalence classes of) product systems of Hilbert spaces 
on the one hand, and $\mathrm{E}_{0}$-semigroups on $B(H)$ on the other.

2. There are several ways to construct a product system from a given $\mathrm{E}_{0}$-semigroup, one of which we mention is the following technique of Arveson: Given the semigroup $\left\{\alpha_{t}\right\}$ on $B(H)$, form the intertwining spaces

$$
E_{t}=\left\{X \in B(H) \mid \forall Y \in B(H): X Y=Y \alpha_{t}(X) \cdot\right\}
$$

A straightforward calculation shows that for $X, Y \in E_{t}$, the operator $X^{*} Y$ must commute with everything in $B(H)$, so it corresponds to a scalar which we define to be $\langle X, Y\rangle$. Furthermore, $E_{t}$ turns out to be complete in this inner product, hence a Hilbert space; moreover, these spaces "tensor" associatively, where for $X \in E_{t}$ and $Y \in E_{s}$ the "tensor product" $X \otimes Y \in E_{t+s}$ is just the composition of operators $X Y$.

3. There is a classification of product systems into types I, II, and III, similar in spirit to the type theory of von Neumann algebras. The classification is based on the notion of a unit for a product system $\left\{E_{t}\right\}$, which is a family of (unit) vectors $u_{t} \in E_{t}$ that follow the given embeddings, that is, such that $u_{t} \otimes u_{s}$ is identified with $u_{s+t}$. If the units "span" the product system in the appropriate sense, it is type I; if there exists at least one unit but they do not span the system, it is type II; if there are no units, it is type III. Type I systems are further classified according to their index, which is a number in $\mathbb{N} \cup\{\infty\}$ defined as the dimension of a certain Hilbert space associated to the set of units, and equal to the index (as defined by Powers in [Pow88]) of the 
associated $\mathrm{E}_{0}$-semigroup.

\subsection{Hilbert $\mathrm{C}^{*}$-Modules and Correspondences}

Given a $\mathrm{C}^{*}$-algebra $A$, a Hilbert $A$-module is a right $A$-module $E$ with an " $A$-valued inner product" such that $E$ is complete in the associated norm. A Hilbert space is precisely a Hilbert $\mathbb{C}$-module. As another notable example, if $X$ is a locally compact Hausdorff space, a vector bundle over $X$ (in which each fiber is a closed subspace of some fixed Hilbert space) is a Hilbert $C(X)$-module; for this reason Hilbert $\mathrm{C}^{*}$-modules are sometimes conceptualized as "noncommutative vector bundles." The basic theory can be found in the seminal papers Pas73, Rie74, [Kas80] and the more recent sources [Lan95], [MT05], and [RW98]. Among the notable features are the replacement of bounded operators on $E$ with the more restrictive notion of adjointable operators, the set of which is denoted $\mathcal{L}(E)$ and forms a $\mathrm{C}^{*}$ algebra; if $A$ is a $\mathrm{W}^{*}$-algebra and $E$ is self-dual (a property not enjoyed by all $\mathrm{C}^{*}$-modules), then $\mathcal{L}(E)$ is a $\mathrm{W}^{*}$-algebra. If $A$ is a $\mathrm{W}^{*}$-algebra and $E$ is not self-dual, one typically works instead with its self-dual completion.

Given $\mathrm{C}^{*}$-algebras $A$ and $B$, a Hilbert $B$-module equipped with a left action of $A$ (by which one means a ${ }^{*}$-homomorphism from $A$ to $\mathcal{L}(E)$ ) is called an $(A, B)$-correspondence. As with balanced tensor products of bimodules over rings, one can form the tensor product (sometimes called the internal tensor product) of an $(A, B)$-correspondence with a $(B, C)$-correspondence to obtain an $(A, C)$ correspondence. In particular, given an $(A, A)$-correspondence $E$, one can form tensor 
powers $E^{\otimes n}$, and thereby also the Fock correspondence $\mathcal{F}(E)=\bigoplus_{n=0}^{\infty} E^{\otimes n}$. More generally, one can form a product system of $(A, A)$-correspondences in a fashion analogous to the last section.

As outlined above, Arveson's intertwining technique produces a product system of Hilbert spaces from an $\mathrm{E}_{0^{-}}$-semigroup on $B(H)$. When one considers an $\mathrm{E}_{0^{-}}$ semigroup on a general von Neumann algebra $\mathcal{M}$, however, the same technique produces a product system of correspondences over the commutant $\mathcal{M}^{\prime}$. Hence, product systems of correspondences arise naturally in the study of $\mathrm{E}_{0}$-semigroups on von Neumann algebras.

The classification theory of product systems of correspondences is more complicated than that of product systems of Hilbert spaces, in part because it is more complicated even to define what a unit is. Following [Ske06], we define a unit to be a family of elements $\xi_{t} \in E_{t}$ which tensor associatively, a unital unit to be one for which $\left\langle\xi_{t}, \xi_{t}\right\rangle=\mathbf{1}$ for all $t$ (this is not automatic even with the hypothesis that $\left\|\xi_{t}\right\|=1$ ), and a central unit to be one for which the left and right actions of $\mathcal{M}$ agree, i.e. $m \cdot \xi_{t}=\xi_{t} \cdot m$ for all $t$ and all $m \in \mathcal{M}$. Central unital units are of particular importance in classification theory; fortunately, it is known that for product systems of von Neumann modules, the existence of a central contractive unit (meaning $\left\langle\xi_{t}, \xi_{t}\right\rangle \leq \mathbf{1}$ for all $t$ ) implies the existence of a central unital unit. A product system having such a unit is called either spatial or non-type-III, though sometimes those terms are distinguished. 


\subsection{Units for Product Systems Associated With Sauvageot Dilations}

In this section we present some calculations toward the construction of a unit for the product system arising from a Sauvageot dilation. For convenience we work with the dilation $\left(\widetilde{\mathfrak{A}},\left\{\widetilde{\sigma}_{t}\right\}\right)$ instead of its quotient $\left(\widehat{\mathfrak{A}},\left\{\widehat{\sigma}_{t}\right\}\right)$.

- Define for each $t \geq 0$ an operator $U_{t} \in B(\mathfrak{H})$ by its action on $\psi(\mathcal{P}) V H$ :

$$
U_{t} \psi\left(\sigma_{t_{1}}\left(i\left(a_{1}\right)\right)\right) \cdots \psi\left(\sigma_{t_{n}}\left(i\left(a_{n}\right)\right)\right) V h=\psi\left(\sigma_{t_{1}+t}\left(i\left(a_{1}\right)\right)\right) \cdots \psi\left(\sigma_{t_{n}+t}\left(i\left(a_{n}\right)\right)\right) V h
$$

- We omit the verification that this extends to a well-defined map on the linear span of $\psi(\mathcal{P}) V H$, and show its contractivity. For simplicity, consider an element of $\psi(\mathcal{P}) V H$, and compute

$$
\begin{aligned}
\left\|U_{t} \psi\left(\sigma_{t_{1}}\left(i\left(a_{1}\right)\right)\right) \cdots \psi\left(\sigma_{t_{n}}\left(i\left(a_{n}\right)\right)\right) V h\right\|^{2} & =\left\|\psi\left(\sigma_{t_{1}+t}\left(i\left(a_{1}\right)\right)\right) \cdots \psi\left(\sigma_{t_{n}+t}\left(i\left(a_{n}\right)\right)\right) V h\right\|^{2} \\
& =\left\langle V^{*} \psi\left(\sigma_{t_{n}+t}\left(i\left(a_{n}\right)\right)^{*} \cdots \sigma_{t_{n}+t}\left(i\left(a_{n}\right)\right)\right) V h, h\right\rangle \\
& =\left\langle\pi \circ \mathbb{E} \circ \sigma_{t}\left(\sigma_{t_{n}}\left(i\left(a_{n}\right)\right)^{*} \cdots \sigma_{t_{n}}\left(i\left(a_{n}\right)\right)\right) h, h\right\rangle \\
& =\left\langle\pi \circ \phi_{t} \circ \mathbb{E}\left(\sigma_{t_{n}}\left(i\left(a_{n}\right)\right)^{*} \cdots \sigma_{t_{n}}\left(i\left(a_{n}\right)\right)\right) h, h\right\rangle \\
& \leq\left\langle\pi \circ \mathbb{E}\left(\sigma_{t_{n}}\left(i\left(a_{n}\right)\right)^{*} \cdots \sigma_{t_{n}}\left(i\left(a_{n}\right)\right)\right) h, h\right\rangle \\
& =\left\|\psi\left(\sigma_{t_{1}}\left(i\left(a_{1}\right)\right)\right) \cdots \psi\left(\sigma_{t_{n}}\left(i\left(a_{n}\right)\right)\right) V h\right\|^{2}
\end{aligned}
$$

since each $\phi_{t}$ is contractive.

- Clearly $U_{t} U_{s}=U_{t+s}$. To show that $\left\{U_{t}\right\}$ is a unit, we need to verify that $U_{t} Y=\widetilde{\sigma}_{t}(Y) U_{t}$ for all $Y \in \widetilde{\mathfrak{A}}$. By the normality of both sides, it suffices to establish this for $Y$ in the weakly dense subalgebra $\psi(\mathfrak{A})$, which reduces again 
to consideration of elements $\psi(\mathcal{P})$. Now letting $\sigma_{t_{1}}\left(i\left(a_{1}\right)\right) \cdots \sigma_{t_{n}}\left(i\left(a_{n}\right)\right)$ be a typical element of $\psi(\mathcal{P})$ and $\sigma_{s_{1}}\left(i\left(b_{1}\right)\right) \cdots \sigma_{s_{m}}\left(i\left(b_{m}\right)\right) V h$ a typical vector in $\mathfrak{H}$,

$$
\begin{aligned}
U_{t} \psi\left(\sigma_{t_{1}}\left(i\left(a_{1}\right)\right)\right. & \left.\cdots \sigma_{t_{n}}\left(i\left(a_{n}\right)\right) \sigma_{s_{1}}\left(i\left(b_{1}\right)\right) \cdots \sigma_{s_{m}}\left(i\left(b_{m}\right)\right)\right) V h \\
& =\psi\left(\sigma_{t_{1}+t}\left(i\left(a_{1}\right)\right) \cdots \sigma_{t_{n}+t}\left(i\left(a_{n}\right)\right) \sigma_{s_{1}+t}\left(i\left(b_{1}\right)\right) \cdots \sigma_{s_{m}+t}\left(i\left(b_{m}\right)\right)\right) V h \\
& =\left[\widetilde{\sigma}_{t}\left(\psi\left(\sigma_{t_{1}}\left(i\left(a_{1}\right)\right) \cdots \sigma_{t_{n}}\left(i\left(a_{n}\right)\right)\right)\right)\right] U_{t}\left[\psi\left(\sigma_{s_{1}}\left(i\left(b_{1}\right)\right) \cdots \sigma_{s_{m}}\left(i\left(b_{m}\right)\right)\right) V h\right]
\end{aligned}
$$

as desired.

- So far we have a contractive unit. To show centrality, consider an operator $X \in \widetilde{\mathfrak{A}}^{\prime}$ and a typical vector $\psi(p) V h$ for $p \in \mathcal{P}$. Note that $X$ commutes with $\psi(p)$ and $\psi\left(\sigma_{t}(p)\right)$, as both are elements of $\widetilde{\mathfrak{A}}$. Then

$$
U_{t} X \psi(p) V h=U_{t} \psi(p) X V h=\psi\left(\sigma_{t}(p)\right) X V h=X \psi\left(\sigma_{t}(p)\right) V h=X U_{t} \psi(p) V h
$$

so that $U_{t} X=X U_{t}$.

The existence of a central contractive unit implies that the dilation semigroup is non-type-III, as referenced above. In particular, this is the case even when the original CP-semigroup happens to be a type III $\mathrm{E}_{0}$-semigroup - a striking result indeed! 


\section{APPENDIX A}

\section{TABLE OF VALUES OF COLLAPSE AND MOMENT FUNCTIONS}

In the tables to come, $\vec{x}_{\ell}$ denotes the tuple $\left(b_{0}, a_{1}, b_{1}, \ldots, a_{\ell}, b_{\ell}\right)$, and $\vec{y}_{\ell}$ the tuple $\left(a_{0}, b_{1}, a_{1}, \ldots, b_{\ell}, a_{\ell}\right)$. 


\section{A.1 Right-Liberation Collapse Functions}

$$
\begin{aligned}
& \operatorname{LC}\left(\vec{x}_{1} ; \emptyset\right)=\vec{x}_{1} \quad \operatorname{LC}\left(\vec{x}_{3} ; \emptyset\right)=\left(b_{0}, a_{1} a_{2} a_{3}, b_{3}\right) \\
& \mathrm{RC}\left(\vec{x}_{1} ; \emptyset\right)=b_{0} \rho\left(a_{1}\right) b_{1} \quad \mathrm{LC}\left(\vec{x}_{3} ;\{1\}\right)=\left(b_{0}, a_{1}, \stackrel{\circ}{b}_{1}, a_{2} a_{3}, b_{3}\right) \\
& \operatorname{RC}\left(\vec{x}_{1} ;\{1\}\right)=\vec{x}_{1} \quad \operatorname{LC}\left(\vec{x}_{3} ;\{2\}\right)=\left(b_{0}, a_{1} a_{2}, \stackrel{\circ}{b}_{2}, a_{3}, b_{3}\right) \\
& \mathrm{UC}\left(\vec{x}_{1} ; \emptyset\right)=-b_{0} \rho\left(a_{1}\right) b_{1} \quad \operatorname{LC}\left(\vec{x}_{3} ;\{1,2\}\right)=\left(b_{0}, a_{1}, \stackrel{\circ}{b}_{1}, a_{2}, \stackrel{\circ}{b}_{2}, a_{3}, b_{3}\right) \\
& \mathrm{UC}\left(\vec{x}_{1} ;\{1\}\right)=\vec{x}_{1} \quad \mathrm{RC}\left(\vec{x}_{3} ; \emptyset\right)=b_{0} \rho\left(a_{1}\right) b_{1} \rho\left(a_{2}\right) b_{2} \rho\left(a_{3}\right) b_{3} \\
& \operatorname{LC}\left(\vec{x}_{2} ; \emptyset\right)=\left(b_{0}, a_{1} a_{2}, b_{1}\right) \quad \operatorname{RC}\left(\vec{x}_{3} ;\{1\}\right)=\left(b_{0}, a_{1}, b_{1} \rho\left(a_{2}\right) b_{2} \rho\left(a_{3}\right) b_{3}\right) \\
& \mathrm{LC}\left(\vec{x}_{2} ;\{1\}\right)=\left(b_{0}, a_{1}, \stackrel{\circ}{b}_{1}, a_{2}, b_{2}\right) \quad \mathrm{RC}\left(\vec{x}_{3} ;\{2\}\right)=\left(b_{0} \rho\left(a_{1}\right) b_{1}, a_{2}, b_{2} \rho\left(a_{3}\right) b_{3}\right) \\
& \operatorname{RC}\left(\vec{x}_{2} ; \emptyset\right)=\left(b_{0} \rho\left(a_{1}\right) b_{1} \rho\left(a_{2}\right) b_{2}\right) \quad \operatorname{RC}\left(\vec{x}_{3} ;\{3\}\right)=\left(b_{0} \rho\left(a_{1}\right) b_{1} \rho\left(a_{2}\right) b_{2}, a_{3}, b_{3}\right) \\
& \operatorname{RC}\left(\vec{x}_{2} ;\{1\}\right)=\left(b_{0}, a_{1}, b_{1} \rho\left(a_{2}\right) b_{2}\right) \quad \operatorname{RC}\left(\vec{x}_{3} ;\{1,2\}\right)=\left(b_{0}, a_{1}, b_{1}, a_{2}, b_{2} \rho\left(a_{3}\right) b_{3}\right) \\
& \operatorname{RC}\left(\vec{x}_{2} ;\{2\}\right)=\left(b_{0} \rho\left(a_{1}\right) b_{1}, a_{2}, b_{2}\right) \quad \operatorname{RC}\left(\vec{x}_{3} ;\{1,3\}\right)=\left(b_{0}, a_{1}, b_{1} \rho\left(a_{2}\right) b_{2}, a_{3}, b_{3}\right) \\
& \mathrm{RC}\left(\vec{x}_{2} ;\{1,2\}\right)=\vec{x}_{2} \quad \operatorname{RC}\left(\vec{x}_{3} ;\{2,3\}\right)=\left(b_{0} \rho\left(a_{1}\right) b_{1}, a_{2}, b_{2}, a_{3}, b_{3}\right) \\
& \mathrm{UC}\left(\vec{x}_{2} ; \emptyset\right)=\left(b_{0} \rho\left(a_{1}\right) b_{1} \rho\left(a_{2}\right) b_{2}\right) \quad \mathrm{RC}\left(\vec{x}_{3} ;\{1,2,3\}\right)=\left(b_{0}, a_{1}, b_{1}, a_{2}, b_{2}, a_{3}, b_{3}\right) \\
& \mathrm{UC}\left(\vec{x}_{2} ;\{1\}\right)=\left(b_{0}, a_{1},-b_{1} \rho\left(a_{2}\right) b_{2}\right) \quad \mathrm{UC}\left(\vec{x}_{3} ; \emptyset\right)=-b_{0} \rho\left(a_{1}\right) b_{1} \rho\left(a_{2}\right) b_{2} \rho\left(a_{3}\right) b_{3} \\
& \mathrm{UC}\left(\vec{x}_{2} ;\{2\}\right)=\left(-b_{0} \rho\left(a_{1}\right) b_{1}, a_{2}, b_{2}\right) \quad \mathrm{UC}\left(\vec{x}_{3} ;\{1\}\right)=\left(b_{0}, a_{1}, b_{1} \rho\left(a_{2}\right) b_{2} \rho\left(a_{3}\right) b_{3}\right) \\
& \mathrm{UC}\left(\vec{x}_{2} ;\{1,2\}\right)=\vec{x}_{2} \quad \mathrm{UC}\left(\vec{x}_{3} ;\{2\}\right)=\left(-b_{0} \rho\left(a_{1}\right) b_{1}, a_{2},-b_{2} \rho\left(a_{3}\right) b_{3}\right) \\
& \mathrm{UC}\left(\vec{x}_{3} ;\{3\}\right)=\left(b_{0} \rho\left(a_{1}\right) b_{1} \rho\left(a_{2}\right) b_{2}, a_{3}, b_{3}\right) \\
& \mathrm{UC}\left(\vec{x}_{3} ;\{1,2\}\right)=\left(b_{0}, a_{1}, b_{1}, a_{2},-b_{2} \rho\left(a_{3}\right) b_{3}\right) \\
& \mathrm{UC}\left(\vec{x}_{3} ;\{1,3\}\right)=\left(b_{0}, a_{1},-b_{1} \rho\left(a_{2}\right) b_{2}, a_{3}, b_{3}\right) \\
& \mathrm{UC}\left(\vec{x}_{3} ;\{2,3\}\right)=\left(-b_{0} \rho\left(a_{1}\right) b_{1}, a_{2}, b_{2}, a_{3}, b_{3}\right) \\
& \mathrm{UC}\left(\vec{x}_{3} ;\{1,2,3\}\right)=\left(b_{0}, a_{1}, b_{1}, a_{2}, b_{2}, a_{3}, b_{3}\right)
\end{aligned}
$$




$$
\begin{aligned}
& \mathrm{LC}\left(\vec{x}_{4} ; \emptyset\right)=\left(b_{0}, a_{1} a_{2} a_{3} a_{4}, b_{4}\right) \\
& \mathrm{LC}\left(\vec{x}_{4} ;\{1\}\right)=\left(b_{0}, a_{1}, \stackrel{\circ}{b_{1}}, a_{2} a_{3} a_{4}, b_{4}\right) \\
& \mathrm{LC}\left(\vec{x}_{4} ;\{2\}\right)=\left(b_{0}, a_{1} a_{2}, \stackrel{\circ}{b}_{2}, a_{3} a_{4}, b_{4}\right) \\
& \mathrm{LC}\left(\vec{x}_{4} ;\{3\}\right)=\left(b_{0}, a_{1} a_{2} a_{3}, \stackrel{\circ}{b}_{3}, a_{4}, b_{4}\right) \\
& \mathrm{LC}\left(\vec{x}_{4} ;\{1,2\}\right)=\left(b_{0}, a_{1}, \stackrel{\circ}{b}_{1}, a_{2}, \stackrel{\circ}{b}_{2}, a_{3} a_{4}, b_{4}\right) \\
& \mathrm{LC}\left(\vec{x}_{4} ;\{1,3\}\right)=\left(b_{0}, a_{1}, \stackrel{\circ}{b}_{1}, a_{2} a_{3}, \stackrel{\circ}{b}_{3}, a_{4}, b_{4}\right) \\
& \operatorname{LC}\left(\vec{x}_{4} ;\{2,3\}\right)=\left(b_{0}, a_{1} a_{2}, \stackrel{\circ}{b}_{2}, a_{3}, \stackrel{\circ}{b}_{3}, a_{4}, b_{4}\right) \\
& \mathrm{LC}\left(\vec{x}_{4} ;\{1,2,3\}\right)=\left(b_{0}, a_{1}, \stackrel{\circ}{b}_{1}, a_{2}, \stackrel{\circ}{b}_{2}, a_{3}, \stackrel{\circ}{b}_{3}, a_{4}, b_{4}\right) \\
& \mathrm{RC}\left(\vec{x}_{4} ; \emptyset\right)=b_{0} \rho\left(a_{1}\right) b_{1} \rho\left(a_{2}\right) b_{2} \rho\left(a_{3}\right) b_{3} \rho\left(a_{4}\right) b_{4} \\
& \mathrm{RC}\left(\vec{x}_{4} ;\{1\}\right)=\left(b_{0}, a_{1}, b_{1} \rho\left(a_{2}\right) b_{2} \rho\left(a_{3}\right) b_{3} \rho\left(a_{4}\right) b_{4}\right) \\
& \mathrm{RC}\left(\vec{x}_{4} ;\{2\}\right)=\left(b_{0} \rho\left(a_{1}\right) b_{1}, a_{2}, b_{2} \rho\left(a_{3}\right) b_{3} \rho\left(a_{4}\right) b_{4}\right) \\
& \mathrm{RC}\left(\vec{x}_{4} ;\{3\}\right)=\left(b_{0} \rho\left(a_{1}\right) b_{1} \rho\left(a_{2}\right) b_{2}, a_{3}, b_{3} \rho\left(a_{4}\right) b_{4}\right) \\
& \mathrm{RC}\left(\vec{x}_{4} ;\{4\}\right)=\left(b_{0} \rho\left(a_{1}\right) b_{1} \rho\left(a_{2}\right) b_{2} \rho\left(a_{3}\right) b_{3}, a_{4}, b_{4}\right) \\
& \mathrm{RC}\left(\vec{x}_{4} ;\{1,2\}\right)=\left(b_{0}, a_{1}, b_{1}, a_{2}, b_{2} \rho\left(a_{3}\right) b_{3} \rho\left(a_{4}\right) b_{4}\right) \\
& \mathrm{RC}\left(\vec{x}_{4} ;\{1,3\}\right)=\left(b_{0}, a_{1}, b_{1} \rho\left(a_{2}\right) b_{2}, a_{3}, b_{3} \rho\left(a_{4}\right) b_{4}\right) \\
& \mathrm{RC}\left(\vec{x}_{4} ;\{1,4\}\right)=\left(b_{0}, a_{1}, b_{1} \rho\left(a_{2}\right) b_{2} \rho\left(a_{3}\right) b_{3}, a_{4}, b_{4}\right) \\
& \mathrm{RC}\left(\vec{x}_{4} ;\{2,3\}\right)=\left(b_{0} \rho\left(a_{1}\right) b_{1}, a_{2}, b_{2}, a_{3}, b_{3} \rho\left(a_{4}\right) b_{4}\right) \\
& \mathrm{RC}\left(\vec{x}_{4} ;\{2,4\}\right)=\left(b_{0} \rho\left(a_{1}\right) b_{1}, a_{2}, b_{2} \rho\left(a_{3}\right) b_{3}, a_{4}, b_{4}\right) \\
& \mathrm{RC}\left(\vec{x}_{4} ;\{3,4\}\right)=\left(b_{0} \rho\left(a_{1}\right) b_{1} \rho\left(a_{2}\right) b_{2}, a_{3}, b_{3}, a_{4}, b_{4}\right)
\end{aligned}
$$




$$
\begin{aligned}
& \mathrm{RC}\left(\vec{x}_{4} ;\{1,2,3\}\right)=\left(b_{0}, a_{1}, b_{1}, a_{2}, b_{2}, a_{3}, b_{3} \rho\left(a_{4}\right) b_{4}\right) \\
& \mathrm{RC}\left(\vec{x}_{4} ;\{1,2,4\}\right)=\left(b_{0}, a_{1}, b_{1}, a_{2}, b_{2} \rho\left(a_{3}\right) b_{3}, a_{4}, b_{4}\right) \\
& \mathrm{RC}\left(\vec{x}_{4} ;\{1,3,4\}\right)=\left(b_{0}, a_{1}, b_{1} \rho\left(a_{2}\right) b_{2}, a_{3}, b_{3}, a_{4}, b_{4}\right) \\
& \mathrm{RC}\left(\vec{x}_{4} ;\{2,3,4\}\right)=\left(b_{0} \rho\left(a_{1}\right) b_{1}, a_{2}, b_{2}, a_{3}, b_{3}, a_{4}, b_{4}\right) \\
& \operatorname{RC}\left(\vec{x}_{4} ;\{1,2,3,4\}\right)=\left(b_{0}, a_{1}, b_{1}, a_{2}, b_{2}, a_{3}, b_{3}, a_{4}, b_{4}\right) \\
& \mathrm{UC}\left(\vec{x}_{4} ; \emptyset\right)=b_{0} \rho\left(a_{1}\right) b_{1} \rho\left(a_{2}\right) b_{2} \rho\left(a_{3}\right) b_{3} \rho\left(a_{4}\right) b_{4} \\
& \mathrm{UC}\left(\vec{x}_{4} ;\{1\}\right)=\left(b_{0}, a_{1},-b_{1} \rho\left(a_{2}\right) b_{2} \rho\left(a_{3}\right) b_{3} \rho\left(a_{4}\right) b_{4}\right) \\
& \mathrm{UC}\left(\vec{x}_{4} ;\{2\}\right)=\left(-b_{0} \rho\left(a_{1}\right) b_{1}, a_{2}, b_{2} \rho\left(a_{3}\right) b_{3} \rho\left(a_{4}\right) b_{4}\right) \\
& \mathrm{UC}\left(\vec{x}_{4} ;\{3\}\right)=\left(b_{0} \rho\left(a_{1}\right) b_{1} \rho\left(a_{2}\right) b_{2}, a_{3},-b_{3} \rho\left(a_{4}\right) b_{4}\right) \\
& \mathrm{UC}\left(\vec{x}_{4} ;\{4\}\right)=\left(-b_{0} \rho\left(a_{1}\right) b_{1} \rho\left(a_{2}\right) b_{2} \rho\left(a_{3}\right) b_{3}, a_{4}, b_{4}\right) \\
& \mathrm{UC}\left(\vec{x}_{4} ;\{1,2\}\right)=\left(b_{0}, a_{1}, b_{1}, a_{2}, b_{2} \rho\left(a_{3}\right) b_{3} \rho\left(a_{4}\right) b_{4}\right) \\
& \mathrm{UC}\left(\vec{x}_{4} ;\{1,3\}\right)=\left(b_{0}, a_{1},-b_{1} \rho\left(a_{2}\right) b_{2}, a_{3},-b_{3} \rho\left(a_{4}\right) b_{4}\right) \\
& \mathrm{UC}\left(\vec{x}_{4} ;\{1,4\}\right)=\left(b_{0}, a_{1}, b_{1} \rho\left(a_{2}\right) b_{2} \rho\left(a_{3}\right) b_{3}, a_{4}, b_{4}\right) \\
& \mathrm{UC}\left(\vec{x}_{4} ;\{2,3\}\right)=\left(-b_{0} \rho\left(a_{1}\right) b_{1}, a_{2}, b_{2}, a_{3},-b_{3} \rho\left(a_{4}\right) b_{4}\right) \\
& \mathrm{UC}\left(\vec{x}_{4} ;\{2,4\}\right)=\left(-b_{0} \rho\left(a_{1}\right) b_{1}, a_{2},-b_{2} \rho\left(a_{3}\right) b_{3}, a_{4}, b_{4}\right) \\
& \mathrm{UC}\left(\vec{x}_{4} ;\{3,4\}\right)=\left(b_{0} \rho\left(a_{1}\right) b_{1} \rho\left(a_{2}\right) b_{2}, a_{3}, b_{3}, a_{4}, b_{4}\right) \\
& \mathrm{UC}\left(\vec{x}_{4} ;\{1,2,3\}\right)=\left(b_{0}, a_{1}, b_{1}, a_{2}, b_{2}, a_{3},-b_{3} \rho\left(a_{4}\right) b_{4}\right) \\
& \mathrm{UC}\left(\vec{x}_{4} ;\{1,2,4\}\right)=\left(b_{0}, a_{1}, b_{1}, a_{2},-b_{2} \rho\left(a_{3}\right) b_{3}, a_{4}, b_{4}\right) \\
& \mathrm{UC}\left(\vec{x}_{4} ;\{1,3,4\}\right)=\left(b_{0}, a_{1},-b_{1} \rho\left(a_{2}\right) b_{2}, a_{3}, b_{3}, a_{4}, b_{4}\right) \\
& \mathrm{UC}\left(\vec{x}_{4} ;\{2,3,4\}\right)=\left(-b_{0} \rho\left(a_{1}\right) b_{1}, a_{2}, b_{2}, a_{3}, b_{3}, a_{4}, b_{4}\right) \\
& \mathrm{UC}\left(\vec{x}_{4} ;\{1,2,3,4\}\right)=\left(b_{0}, a_{1}, b_{1}, a_{2}, b_{2}, a_{3}, b_{3}, a_{4}, b_{4}\right)
\end{aligned}
$$




\section{A.2 Right-Liberation Moment Functions}

$$
\begin{aligned}
& \operatorname{RM}\left(\vec{x}_{1}\right)=b_{0} \rho\left(a_{1}\right) b_{1} \\
& \operatorname{LM}\left(\vec{x}_{1}\right)=b_{0} \rho\left(a_{1}\right) b_{1} \\
& \mathrm{UM}\left(\vec{x}_{1}\right)=0 \\
& \operatorname{RM}\left(\vec{x}_{2}\right)=b_{0} \rho\left(a_{1}\right) b_{1} \rho\left(a_{2}\right) b_{2} \\
& \operatorname{LM}\left(\vec{x}_{2}\right)=\nu\left(b_{1}\right) b_{0}\left[\rho\left(a_{1} a_{2}\right)-\rho\left(a_{1}\right) \rho\left(a_{2}\right)\right] b_{2}+b_{0} \rho\left(a_{1}\right) b_{1} \rho\left(a_{2}\right) b_{2} \\
& \mathrm{UM}\left(\vec{x}_{2}\right)=\nu\left(b_{1}\right) b_{0}\left[\rho\left(a_{1} a_{2}\right)-\rho\left(a_{1}\right) \rho\left(a_{2}\right)\right] b_{2} \\
& \operatorname{RM}\left(\vec{x}_{3}\right)=b_{0} \rho\left(a_{1}\right) b_{1} \rho\left(a_{2}\right) b_{2} \rho\left(a_{3}\right) b_{3}+\nu\left(b_{1}\right) b_{0}\left[\rho\left(a_{1} a_{2}\right)-\rho\left(a_{1}\right) \rho\left(a_{2}\right)\right] b_{3} \\
& +\nu\left(b_{1} \rho\left(a_{2}\right) b_{2}\right) b_{0}\left[\rho\left(a_{1} a_{3}\right)-\rho\left(a_{1}\right) \rho\left(a_{3}\right)\right] b_{3}+\nu\left(b_{2}\right) b_{0}\left[\rho\left(a_{2} a_{3}\right)-\rho\left(a_{2}\right) \rho\left(a_{3}\right)\right] b_{3} \\
& \operatorname{LM}\left(\vec{x}_{3}\right)=\nu\left(b_{1}\right) \nu\left(b_{2}\right) b_{0} \rho\left(a_{1} a_{2} a_{3}\right) b_{3}+\nu\left(b_{2}\right) b_{0} \rho\left(a_{1}\right) \stackrel{\circ}{b}_{1} \rho\left(a_{2} a_{3}\right) b_{3} \\
& +\nu\left(b_{1}\right) b_{0} \rho\left(a_{1} a_{2}\right) \stackrel{\circ}{b}_{2} \rho\left(a_{3}\right) b_{3}+b_{0} \rho\left(a_{1}\right) \stackrel{\circ}{b}_{1} \rho\left(a_{2}\right) \stackrel{\circ}{b}_{2} \rho\left(a_{3}\right) b_{3} \\
& +\nu\left(\stackrel{\circ}{b}_{1} \rho\left(a_{2}\right) \stackrel{\circ}{b}_{2}\right) b_{0}\left[\rho\left(a_{1} a_{3}\right)-\rho\left(a_{1}\right) \rho\left(a_{3}\right)\right] b_{3} \\
& =b_{0} \rho\left(a_{1}\right) b_{1} \rho\left(a_{2}\right) b_{2} \rho\left(a_{3}\right) b_{3} \\
& +\nu\left(b_{1}\right) \nu\left(b_{2}\right) b_{0}\left[\rho\left(a_{1} a_{2} a_{3}\right)-\rho\left(a_{1}\right) \rho\left(a_{2} a_{3}\right)-\rho\left(a_{1} a_{2}\right) \rho\left(a_{3}\right)+\rho\left(a_{1}\right) \rho\left(a_{2}\right) \rho\left(a_{3}\right)\right] b_{3} \\
& +\nu\left(b_{1}\right) b_{0}\left[\rho\left(a_{1} a_{2}\right)-\rho\left(a_{1}\right) \rho\left(a_{2}\right)\right] b_{2} \rho\left(a_{3}\right) b_{3}+\nu\left(b_{2}\right) b_{0} \rho\left(a_{1}\right) b_{1}\left[\rho\left(a_{2} a_{3}\right)-\rho\left(a_{2}\right) \rho\left(a_{3}\right)\right] b_{3} \\
& +\left[\nu\left(b_{1}\right) \nu\left(b_{2}\right) \nu\left(\rho\left(a_{2}\right)\right)-\nu\left(b_{1}\right) \nu\left(\rho\left(a_{2}\right) b_{2}\right)-\nu\left(b_{1} \rho\left(a_{2}\right)\right) \nu\left(b_{2}\right)\right. \\
& \left.+\nu\left(b_{1} \rho\left(a_{2}\right) b_{2}\right)\right] b_{0}\left[\rho\left(a_{1} a_{3}\right)-\rho\left(a_{1}\right) \rho\left(a_{3}\right)\right] b_{3} \\
& \mathrm{UM}\left(\vec{x}_{3}\right)=\nu\left(b_{1}\right) \nu\left(b_{2}\right) b_{0}\left[\rho\left(a_{1} a_{2} a_{3}\right)-\rho\left(a_{1}\right) \rho\left(a_{2} a_{3}\right)-\rho\left(a_{1} a_{2}\right) \rho\left(a_{3}\right)+\rho\left(a_{1}\right) \rho\left(a_{2}\right) \rho\left(a_{3}\right)\right] b_{3} \\
& +\left[\nu\left(b_{1}\right) \nu\left(b_{2}\right) \nu\left(\rho\left(a_{2}\right)\right)-\nu\left(b_{1}\right) \nu\left(\rho\left(a_{2}\right) b_{2}\right)-\nu\left(b_{1} \rho\left(a_{2}\right)\right) \nu\left(b_{2}\right)\right] b_{0}\left[\rho\left(a_{1} a_{3}\right)-\rho\left(a_{1}\right) \rho\left(a_{3}\right)\right] b_{3}
\end{aligned}
$$




$$
\begin{aligned}
& \operatorname{RM}\left(\vec{x}_{4}\right)=b_{0} \rho\left(a_{1}\right) b_{1} \rho\left(a_{2}\right) b_{2} \rho\left(a_{3}\right) b_{3} \rho\left(a_{4}\right) b_{4}+\nu\left(b_{1}\right) b_{0}\left[\rho\left(a_{1} a_{2}\right)-\rho\left(a_{1}\right) \rho\left(a_{2}\right)\right] b_{2} \rho\left(a_{3}\right) b_{3} \rho\left(a_{4}\right) b_{4} \\
& +\nu\left(b_{1} \rho\left(a_{2}\right) b_{2}\right) b_{0}\left[\rho\left(a_{1} a_{3}\right)-\rho\left(a_{1}\right) \rho\left(a_{3}\right)\right] b_{3} \rho\left(a_{4}\right) b_{4} \\
& +\nu\left(b_{1} \rho\left(a_{2}\right) b_{2} \rho\left(a_{3}\right) b_{3}\right) b_{0}\left[\rho\left(a_{1} a_{4}\right)-\rho\left(a_{1}\right) \rho\left(a_{4}\right)\right] b_{4} \\
& +\nu\left(b_{2}\right) b_{0} \rho\left(a_{1}\right) b_{1}\left[\rho\left(a_{2} a_{3}\right)-\rho\left(a_{2}\right) \rho\left(a_{3}\right)\right] b_{3} \rho\left(a_{4}\right) b_{4} \\
& +\nu\left(b_{2} \rho\left(a_{3}\right) b_{3}\right) b_{0} \rho\left(a_{1}\right) b_{1}\left[\rho\left(a_{2} a_{4}\right)-\rho\left(a_{2}\right) \rho\left(a_{4}\right)\right] b_{4} \\
& +\nu\left(b_{3}\right) b_{0} \rho\left(a_{1}\right) b_{1} \rho\left(a_{2}\right) b_{2}\left[\rho\left(a_{3} a_{4}\right)-\rho\left(a_{3}\right) \rho\left(a_{4}\right)\right] b_{4} \\
& +\nu\left(b_{1} \rho\left(a_{2}\right) b_{2}\right) \nu\left(b_{3}\right) b_{0}\left[\rho\left(a_{1} a_{3} a_{4}\right)-\rho\left(a_{1}\right) \rho\left(a_{3} a_{4}\right)-\rho\left(a_{1} a_{3}\right) \rho\left(a_{4}\right)+\rho\left(a_{1}\right) \rho\left(a_{3}\right) \rho\left(a_{4}\right)\right] b_{4} \\
& +\left[\nu\left(b_{1} \rho\left(a_{2}\right) b_{2}\right) \nu\left(b_{3}\right) \nu\left(\rho\left(a_{3}\right)\right)-\nu\left(b_{1} \rho\left(a_{2}\right) b_{2}\right) \nu\left(\rho\left(a_{3}\right) b_{3}\right)\right. \\
& \left.-\nu\left(b_{1} \rho\left(a_{2}\right) b_{2} \rho\left(a_{3}\right)\right) \nu\left(b_{3}\right)\right] b_{0}\left[\rho\left(a_{1} a_{4}\right)-\rho\left(a_{1}\right) \rho\left(a_{4}\right)\right] b_{4} \\
& +\nu\left(b_{1}\right) \nu\left(b_{2} \rho\left(a_{3}\right) b_{3}\right) b_{0}\left[\rho\left(a_{1} a_{2} a_{4}\right)-\rho\left(a_{1}\right) \rho\left(a_{2} a_{4}\right)-\rho\left(a_{1} a_{2}\right) \rho\left(a_{4}\right)+\rho\left(a_{1}\right) \rho\left(a_{2}\right) \rho\left(a_{4}\right)\right] b_{4} \\
& +\left[\nu\left(b_{1}\right) \nu\left(b_{2} \rho\left(a_{3}\right) b_{3}\right) \nu\left(\rho\left(a_{2}\right)\right)-\nu\left(b_{1}\right) \nu\left(\rho\left(a_{2}\right) b_{2} \rho\left(a_{3}\right) b_{3}\right)\right. \\
& \left.-\nu\left(b_{1} \rho\left(a_{2}\right)\right) \nu\left(b_{2} \rho\left(a_{3}\right) b_{3}\right)\right] b_{0}\left[\rho\left(a_{1} a_{4}\right)-\rho\left(a_{1}\right) \rho\left(a_{4}\right)\right] b_{4} \\
& +\nu\left(b_{1}\right) \nu\left(b_{2}\right) b_{0}\left[\rho\left(a_{1} a_{2} a_{3}\right)-\rho\left(a_{1}\right) \rho\left(a_{2} a_{3}\right)-\rho\left(a_{1} a_{2}\right) \rho\left(a_{3}\right)+\rho\left(a_{1}\right) \rho\left(a_{2}\right) \rho\left(a_{3}\right)\right] b_{3} \rho\left(a_{4}\right) b_{4} \\
& +\left[\nu\left(b_{1}\right) \nu\left(b_{2}\right) \nu\left(\rho\left(a_{2}\right)\right)-\nu\left(b_{1}\right) \nu\left(\rho\left(a_{2}\right) b_{2}\right)\right. \\
& \left.-\nu\left(b_{1} \rho\left(a_{2}\right)\right) \nu\left(b_{2}\right)\right] b_{0}\left[\rho\left(a_{1} a_{3}\right)-\rho\left(a_{1}\right) \rho\left(a_{3}\right)\right] b_{3} \rho\left(a_{4}\right) b_{4} \\
& +\nu\left(b_{2}\right) \nu\left(b_{3}\right) b_{0} \rho\left(a_{1}\right) b_{1}\left[\rho\left(a_{2} a_{3} a_{4}\right)-\rho\left(a_{2}\right) \rho\left(a_{3} a_{4}\right)-\rho\left(a_{2} a_{3}\right) \rho\left(a_{4}\right)+\rho\left(a_{2}\right) \rho\left(a_{3}\right) \rho\left(a_{4}\right)\right] b_{4} \\
& +\left[\nu\left(b_{2}\right) \nu\left(b_{3}\right) \nu\left(\rho\left(a_{3}\right)\right)-\nu\left(b_{2}\right) \nu\left(\rho\left(a_{3}\right) b_{3}\right)\right. \\
& \left.-\nu\left(b_{2} \rho\left(a_{3}\right)\right) \nu\left(b_{3}\right)\right] b_{0} \rho\left(a_{1}\right) b_{1}\left[\rho\left(a_{2} a_{4}\right)-\rho\left(a_{2}\right) \rho\left(a_{4}\right)\right] b_{4}
\end{aligned}
$$




$$
\begin{aligned}
& \operatorname{LM}\left(\vec{x}_{4}\right)=\nu\left(b_{1}\right) \nu\left(b_{2}\right) \nu\left(b_{3}\right) b_{0} \rho\left(a_{1} a_{2} a_{3} a_{4}\right) b_{4}+\nu\left(b_{2}\right) \nu\left(b_{3}\right) b_{0} \rho\left(a_{1}\right) \stackrel{\circ}{b}_{1} \rho\left(a_{2} a_{3} a_{4}\right) b_{4} \\
& +\nu\left(b_{1}\right) \nu\left(b_{3}\right) b_{0} \rho\left(a_{1} a_{2}\right) \stackrel{\circ}{b}_{2} \rho\left(a_{3} a_{4}\right) b_{4}+\nu\left(b_{1}\right) \nu\left(b_{2}\right) b_{0} \rho\left(a_{1} a_{2} a_{3}\right) \stackrel{\circ}{b}_{3} \rho\left(a_{4}\right) b_{4} \\
& +\nu\left(b_{3}\right) b_{0} \rho\left(a_{1}\right) \stackrel{\circ}{b}_{1} \rho\left(a_{2}\right) \stackrel{\circ}{b}_{2} \rho\left(a_{3} a_{4}\right) b_{4}+\nu\left(\stackrel{\circ}{b}_{1} \rho\left(a_{2}\right) \stackrel{\circ}{b}_{2}\right) \nu\left(b_{3}\right) b_{0}\left[\rho\left(a_{1} a_{3} a_{4}\right)-\rho\left(a_{1}\right) \rho\left(a_{3} a_{4}\right)\right] b_{4} \\
& +\nu\left(b_{2}\right) b_{0} \rho\left(a_{1}\right) \stackrel{\circ}{b}_{1} \rho\left(a_{2} a_{3}\right) \stackrel{\circ}{3}_{3} \rho\left(a_{4}\right) b_{4}+\nu\left(\stackrel{\circ}{b}_{1} \rho\left(a_{2} a_{3}\right) \stackrel{\circ}{b}_{3}\right) \nu\left(b_{2}\right) b_{0}\left[\rho\left(a_{1} a_{4}\right)-\rho\left(a_{1}\right) \rho\left(a_{4}\right)\right] b_{4} \\
& +\nu\left(b_{1}\right) b_{0} \rho\left(a_{1} a_{2}\right) \stackrel{\circ}{b}_{2} \rho\left(a_{3}\right) \stackrel{\circ}{b}_{3} \rho\left(a_{4}\right) b_{4}+\nu\left(b_{1}\right) \nu\left(\stackrel{\circ}{b}_{2} \rho\left(a_{3}\right) \stackrel{\circ}{b}_{3}\right) b_{0}\left[\rho\left(a_{1} a_{2} a_{4}\right)-\rho\left(a_{1} a_{2}\right) \rho\left(a_{4}\right)\right] b_{4} \\
& +b_{0} \rho\left(a_{1}\right) \stackrel{\circ}{b}_{1} \rho\left(a_{2}\right) \stackrel{\circ}{b}_{2} \rho\left(a_{3}\right) \stackrel{\circ}{b}_{3} \rho\left(a_{4}\right) b_{4} \\
& +\nu\left(\stackrel{\circ}{b}_{1} \rho\left(a_{2}\right) \stackrel{\circ}{b}_{2}\right) b_{0}\left[\rho\left(a_{1} a_{3}\right)-\rho\left(a_{1}\right) \rho\left(a_{3}\right)\right] \stackrel{\circ}{3}_{3} \rho\left(a_{4}\right) b_{4} \\
& +\nu\left(\stackrel{\circ}{b}_{1} \rho\left(a_{2}\right) \stackrel{\circ}{b}_{2} \rho\left(a_{3}\right) \stackrel{\circ}{b}_{3}\right) b_{0}\left[\rho\left(a_{1} a_{4}\right)-\rho\left(a_{1}\right) \rho\left(a_{4}\right)\right] b_{4} \\
& +\nu\left(\stackrel{\circ}{b}_{2} \rho\left(a_{3}\right) \stackrel{\circ}{b}_{3}\right) b_{0} \rho\left(a_{1}\right) \stackrel{\circ}{b}_{1}\left[\rho\left(a_{2} a_{4}\right)-\rho\left(a_{2}\right) \rho\left(a_{4}\right)\right] b_{4} \\
& -\nu\left(\stackrel{\circ}{b}_{1} \rho\left(a_{2}\right) \stackrel{\circ}{b}_{2}\right) \nu\left(\rho\left(a_{3}\right) \stackrel{\circ}{b}_{3}\right) b_{0}\left[\rho\left(a_{1} a_{4}\right)-\rho\left(a_{1}\right) \rho\left(a_{4}\right)\right] b_{4} \\
& -\nu\left(\stackrel{\circ}{b}_{1} \rho\left(a_{2}\right)\right) \nu\left(\stackrel{\circ}{b}_{2} \rho\left(a_{3}\right) \stackrel{\circ}{b}_{3}\right) b_{0}\left[\rho\left(a_{1} a_{4}\right)-\rho\left(a_{1}\right) \rho\left(a_{4}\right)\right] b_{4}
\end{aligned}
$$




$$
\begin{aligned}
& \operatorname{LM}\left(\vec{x}_{4}\right)=b_{0} \rho\left(a_{1}\right) b_{1} \rho\left(a_{2}\right) b_{2} \rho\left(a_{3}\right) b_{3} \rho\left(a_{4}\right) b_{4}+\nu\left(b_{1}\right) \nu\left(b_{2}\right) \nu\left(b_{3}\right) b_{0} \rho_{[1,2,3,4]}(a) b_{4} \\
& +\nu\left(b_{1}\right) \nu\left(b_{2}\right) \nu\left(b_{3}\right) \nu\left(\rho\left(a_{2}\right)\right) b_{0} \rho_{[1,3,4]}(a) b_{4}+\nu\left(b_{1}\right) \nu\left(b_{2}\right) \nu\left(b_{3}\right) \nu\left(\rho\left(a_{3}\right)\right) b_{0} \rho_{[1,2,4]}(a) b_{4} \\
& +\nu\left(b_{1}\right) \nu\left(b_{3}\right) \nu\left(b_{3}\right)\left[\nu\left(\rho\left(a_{2} a_{3}\right)\right)+2 \nu\left(\rho\left(a_{2}\right)\right) \nu\left(\rho\left(a_{3}\right)\right)-\nu\left(\rho\left(a_{2}\right) \rho\left(a_{3}\right)\right)\right] b_{0} \rho_{[1,4]}(a) b_{4} \\
& +\nu\left(b_{1}\right) \nu\left(b_{2}\right) \nu\left(\rho\left(a_{2}\right)\right) b_{0} \rho_{[1,3]}(a) b_{3} \rho\left(a_{4}\right) b_{4}-\nu\left(b_{1}\right) \nu\left(b_{2}\right) \nu\left(\rho\left(a_{3}\right) b_{3}\right) b_{0} \rho_{[1,2,4]}(a) b_{4} \\
& -\nu\left(b_{1}\right) \nu\left(b_{2}\right)\left[\nu\left(\rho_{[2,3]}(a) b_{3}\right)-2 \nu\left(\rho\left(a_{2}\right)\right) \nu\left(\rho\left(a_{3}\right) b_{3}\right)\right] b_{0} \rho_{[1,4]}(a) b_{4} \\
& +\nu\left(b_{1}\right) \nu\left(b_{3}\right) b_{0} \rho_{[1,2]}(a) b_{2} \rho_{[3,4]}(a) b_{4}-\nu\left(b_{1}\right) \nu\left(b_{3}\right) \nu\left(\rho\left(a_{2}\right) b_{2}\right) \rho_{[1,3,4]}(a) b_{4} \\
& -\nu\left(b_{1}\right) \nu\left(b_{3}\right) \nu\left(b_{2} \rho\left(a_{3}\right)\right) b_{0} \rho_{[1,2,4]} b_{4} \\
& +\nu\left(b_{1}\right) \nu\left(b_{3}\right)\left[\nu\left(\rho\left(a_{2}\right) b_{2} \rho\left(a_{3}\right)\right)-\nu\left(\rho\left(a_{2}\right) b_{2}\right) \nu\left(\rho\left(a_{3}\right)\right)-\nu\left(\rho\left(a_{2}\right)\right) \nu\left(b_{2} \rho\left(a_{3}\right)\right)\right] b_{0} \rho_{[1,4]} b_{4} \\
& +\nu\left(b_{2}\right) \nu\left(b_{3}\right) b_{0} \rho\left(a_{1}\right) b_{1} \rho_{[2,3,4]}(a) b_{4}-\nu\left(b_{2}\right) \nu\left(b_{3}\right) \nu\left(b_{1} \rho\left(a_{2}\right)\right) b_{0} \rho_{[1,3,4]} b_{4} \\
& -\nu\left(b_{2}\right) \nu\left(b_{3}\right)\left[\nu\left(b_{1} \rho_{[2,3]}(a)\right)+2 \nu\left(b_{1} \rho\left(a_{2}\right)\right) \nu\left(\rho\left(a_{3}\right)\right)\right] b_{0} \rho_{[1,4]} b_{4} \\
& +\nu\left(b_{1}\right) b_{0} \rho_{[1,2]}(a) b_{2} \rho\left(a_{3}\right) b_{3} \rho\left(a_{4}\right) b_{4}-\nu\left(b_{1}\right) \nu\left(\rho\left(a_{2}\right) b_{2}\right) b_{0} \rho_{[1,3]}(a) b_{3} \rho\left(a_{4}\right) b_{4} \\
& +\nu\left(b_{1}\right) \nu\left(b_{2} \rho\left(a_{3}\right) b_{3}\right) b_{0} \rho_{[1,2,4]}(a) b_{4} \\
& -\nu\left(b_{1}\right)\left[\nu\left(\rho\left(a_{2}\right) b_{2} \rho\left(a_{3}\right) b_{3}\right)-\nu\left(\rho\left(a_{2}\right)\right) \nu\left(b_{2} \rho\left(a_{3}\right) b_{3}\right)-\nu\left(\rho\left(a_{2}\right) b_{2}\right) \nu\left(\rho\left(a_{3}\right) b_{3}\right)\right] b_{0} \rho_{[1,4]}(a) b_{4} \\
& +\nu\left(b_{2}\right) b_{0} \rho\left(a_{1}\right) b_{1} \rho_{[2,3]}(a) b_{3} \rho\left(a_{4}\right) b_{4}-\nu\left(b_{2}\right) \nu\left(b_{1} \rho\left(a_{2}\right)\right) b_{0} \rho_{[1,3]}(a) b_{3} \rho\left(a_{4}\right) b_{4} \\
& -\nu\left(b_{2}\right) \nu\left(\rho\left(a_{3}\right) b_{3}\right) b_{0} \rho\left(a_{1}\right) b_{1} \rho_{[2,4]}(a) b_{4} \\
& +\nu\left(b_{2}\right)\left[\nu\left(b_{1} \rho_{[2,3]}(a) b_{3}\right)+2 \nu\left(b_{1} \rho\left(a_{2}\right)\right) \nu\left(\rho\left(a_{3}\right) b_{3}\right)\right] b_{0} \rho_{[1,4]} b_{4} \\
& +\nu\left(b_{3}\right) b_{0} \rho\left(a_{1}\right) b_{1} \rho\left(a_{2}\right) b_{2} \rho_{[3,4]} b_{4}+\nu\left(b_{3}\right) \nu\left(b_{1} \rho\left(a_{2}\right) b_{2}\right) b_{0} \rho_{[1,3,4]}(a) b_{4} \\
& -\nu\left(b_{3}\right) \nu\left(b_{2} \rho\left(a_{3}\right)\right) b_{0} \rho\left(a_{1}\right) b_{1} \rho_{[2,4]}(a) b_{4} \\
& -\nu\left(b_{3}\right)\left[\nu\left(b_{1} \rho\left(a_{2}\right) b_{2} \rho\left(a_{3}\right)\right)-\nu\left(b_{1} \rho\left(a_{2}\right) b_{2}\right) \nu\left(\rho\left(a_{3}\right)\right)-\nu\left(b_{1} \rho\left(a_{2}\right)\right) \nu\left(b_{2} \rho\left(a_{3}\right)\right)\right] b_{0} \rho_{[1,4]} b_{4} \\
& +\left[\nu\left(b_{1} \rho\left(a_{2}\right) b_{2} \rho\left(a_{3}\right) b_{3}\right)-\nu\left(b_{1} \rho\left(a_{2}\right) b_{2}\right) \nu\left(\rho\left(a_{3}\right) b_{3}\right)-\nu\left(b_{1} \rho\left(a_{2}\right)\right) \nu\left(b_{2} \rho\left(a_{3}\right) b_{3}\right)\right] b_{0} \rho_{[1,4]} b_{4} \\
& +\nu\left(b_{1} \rho\left(a_{2}\right) b_{2}\right) b_{0} \rho_{[1,3]}(a) b_{3} \rho\left(a_{4}\right) b_{4}+\nu\left(b_{2} \rho\left(a_{3}\right) b_{3}\right) b_{0} \rho\left(a_{1}\right) b_{1} \rho_{[2,4]}(a) b_{4}
\end{aligned}
$$




\section{A.3 Left-Liberation Collapse Functions}

$$
\begin{aligned}
& \mathrm{LC}^{\prime}\left(\vec{y}_{1} ; \emptyset\right)=a_{0} a_{1} \quad \operatorname{LC}^{\prime}\left(\vec{y}_{3} ; \emptyset\right)=a_{0} a_{1} a_{2} a_{3} \\
& \mathrm{LC}^{\prime}\left(\vec{y}_{1} ;\{1\}\right)=\left(a_{0}, \stackrel{\circ}{b}_{1}, a_{1}\right) \quad \operatorname{LC}^{\prime}\left(\vec{y}_{3} ;\{1\}\right)=\left(a_{0}, \stackrel{\circ}{b}_{1}, a_{1} a_{2} a_{3}\right) \\
& \mathrm{RC}^{\prime}\left(\vec{y}_{1} ; \emptyset\right)=\vec{y}_{1} \quad \mathrm{LC}^{\prime}\left(\vec{y}_{3} ;\{2\}\right)=\left(a_{0} a_{1}, \stackrel{\circ}{b_{2}}, a_{2} a_{3}\right) \\
& \mathrm{UC}^{\prime}\left(\vec{y}_{1} ; \emptyset\right)=\vec{y}_{1} \quad \mathrm{LC}^{\prime}\left(\vec{y}_{3} ;\{3\}\right)=\left(a_{0} a_{1} a_{2}, \stackrel{\circ}{b}_{3}, a_{3}\right) \\
& \mathrm{LC}^{\prime}\left(\vec{y}_{2} ; \emptyset\right)=a_{0} a_{1} a_{2} \quad \operatorname{LC}^{\prime}\left(\vec{y}_{3} ;\{1,2\}\right)=\left(a_{0}, \stackrel{\circ}{b}, a_{1}, \stackrel{\circ}{b}_{2}, a_{2} a_{3}\right) \\
& \mathrm{LC}^{\prime}\left(\vec{y}_{2} ;\{1\}\right)=\left(a_{0}, \stackrel{\circ}{b}, a_{1} a_{2}\right) \quad \operatorname{LC}^{\prime}\left(\vec{y}_{3} ;\{1,3\}\right)=\left(a_{0}, \stackrel{\circ}{b}_{1}, a_{1} a_{2}, \stackrel{\circ}{b}_{3}, a_{3}\right) \\
& \operatorname{LC}^{\prime}\left(\vec{y}_{2} ;\{2\}\right)=\left(a_{0} a_{1}, \stackrel{\circ}{b}_{2}, a_{2}\right) \quad \operatorname{LC}^{\prime}\left(\vec{y}_{3} ;\{2,3\}\right)=\left(a_{0} a_{1}, \stackrel{\circ}{b}_{2}, a_{2}, \stackrel{\circ}{b}_{3}, a_{3}\right) \\
& \operatorname{LC}^{\prime}\left(\vec{y}_{2} ;\{1,2\}\right)=\left(a_{0}, \stackrel{\circ}{b}_{1}, a_{1}, \stackrel{\circ}{b}_{2}, a_{2}\right) \quad \operatorname{LC}^{\prime}\left(\vec{y}_{3} ;\{1,2,3\}\right)=\left(a_{0}, \stackrel{\circ}{b}_{1}, a_{1}, \stackrel{\circ}{b}_{2}, a_{2}, \stackrel{\circ}{b}_{3}, a_{3}\right) \\
& \mathrm{RC}^{\prime}\left(\vec{y}_{2} ; \emptyset\right)=\left(a_{0}, b_{1} \rho\left(a_{1}\right) b_{2}, a_{2}\right) \quad \operatorname{RC}^{\prime}\left(\vec{y}_{3} ; \emptyset\right)=\left(a_{0}, b_{1} \rho\left(a_{1}\right) b_{2} \rho\left(a_{2}\right) b_{3}, a_{3}\right) \\
& \operatorname{RC}^{\prime}\left(\vec{y}_{2} ;\{1\}\right)=\vec{y}_{2} \quad \operatorname{RC}^{\prime}\left(\vec{y}_{3} ;\{1\}\right)=\left(a_{0}, b_{1}, a_{1}, b_{2} \rho\left(a_{2}\right) b_{3}, a_{3}\right) \\
& \mathrm{UC}^{\prime}\left(\vec{y}_{2} ; \emptyset\right)=\left(a_{0},-b_{1} \rho\left(a_{1}\right) b_{2}, a_{2}\right) \quad \mathrm{RC}^{\prime}\left(\vec{y}_{3} ;\{2\}\right)=\left(a_{0}, b_{1} \rho\left(a_{1}\right) b_{2}, a_{2}, b_{3}, a_{3}\right) \\
& \mathrm{UC}^{\prime}\left(\vec{y}_{2} ;\{1\}\right)=\vec{y}_{2} \quad \operatorname{RC}^{\prime}\left(\vec{y}_{3} ;\{1,2\}\right)=\vec{y}_{3} \\
& \mathrm{UC}^{\prime}\left(\vec{y}_{3} ; \emptyset\right)=\left(a_{0}, b_{1} \rho\left(a_{1}\right) b_{2} \rho\left(a_{2}\right) b_{3}, a_{3}\right) \\
& \mathrm{UC}^{\prime}\left(\vec{y}_{3} ;\{1\}\right)=\left(a_{0}, b_{1}, a_{1},-b_{2} \rho\left(a_{2}\right) b_{3}, a_{3}\right) \\
& \mathrm{UC}^{\prime}\left(\vec{y}_{3} ;\{2\}\right)=\left(a_{0},-b_{1} \rho\left(a_{1}\right) b_{2}, a_{2}, b_{3}, a_{3}\right) \\
& \mathrm{UC}^{\prime}\left(\vec{y}_{3} ;\{1,2\}\right)=\vec{y}_{3}
\end{aligned}
$$




\section{A.4 Left-Liberation Moment Functions}

$$
\begin{aligned}
& \operatorname{RM}^{\prime}\left(\vec{y}_{1}\right)=0 \\
& \operatorname{LM}^{\prime}\left(\vec{y}_{1}\right)=\nu\left(b_{1}\right) a_{0} a_{1} \\
& \mathrm{UM}^{\prime}\left(\vec{y}_{1}\right)=\nu\left(b_{1}\right) a_{0} a_{1} \\
& \operatorname{RM}^{\prime}\left(\vec{y}_{2}\right)=\nu\left(b_{1} \rho\left(a_{1}\right) b_{2}\right) a_{0} a_{2} \\
& \operatorname{LM}^{\prime}\left(\vec{y}_{2}\right)=\nu\left(b_{1}\right) \nu\left(b_{2}\right) a_{0} a_{1} a_{2}+\nu\left(\stackrel{\circ}{b}_{1} \rho\left(a_{1}\right) \stackrel{\circ}{b}_{2}\right) a_{0} a_{2} \\
& =\nu\left(b_{1}\right) \nu\left(b_{2}\right) a_{0} a_{1} a_{2}+\left[\nu\left(b_{1} \rho\left(a_{1}\right) b_{2}\right)-\nu\left(b_{1}\right) \nu\left(\rho\left(a_{1}\right) b_{2}\right)\right. \\
& \left.-\nu\left(b_{1} \rho\left(a_{1}\right)\right)+\nu\left(b_{1}\right) \nu\left(\rho\left(a_{1}\right)\right) \nu\left(b_{2}\right)\right] a_{0} a_{2} \\
& \mathrm{UM}^{\prime}\left(\vec{y}_{2}\right)=\nu\left(b_{1}\right) \nu\left(b_{2}\right) a_{0} a_{1} a_{2}+\left[\nu\left(\stackrel{\circ}{b}_{1} \rho\left(a_{1}\right) \stackrel{\circ}{b}_{2}\right)-\nu\left(b_{1} \rho\left(a_{1}\right) b_{2}\right)\right] a_{0} a_{2} \\
& =\nu\left(b_{1}\right) \nu\left(b_{2}\right) a_{0} a_{1} a_{2}+\left[\nu\left(b_{1}\right) \nu\left(\rho\left(a_{1}\right)\right) \nu\left(b_{2}\right)-\nu\left(b_{1}\right) \nu\left(\rho\left(a_{1}\right) b_{2}\right)-\nu\left(b_{1} \rho\left(a_{1}\right)\right)\right] a_{0} a_{2} \\
& \operatorname{RM}^{\prime}\left(\vec{y}_{3}\right)=\nu\left(b_{1}\right) \nu\left(b_{2} \rho\left(a_{2}\right) b_{3}\right) a_{0} a_{1} a_{3}+\nu\left(b_{1} \rho\left(a_{1}\right) b_{2}\right) \nu\left(b_{3}\right) a_{0} a_{2} a_{3} \\
& +\left[\nu\left(b_{1} \rho\left(a_{1}\right) b_{2} \rho\left(a_{2}\right) b_{3}\right)-\nu\left(b_{1} \rho\left(a_{2}\right)\right) \nu\left(b_{2} \rho\left(a_{2}\right) b_{3}\right)-\nu\left(b_{1}\right) \nu\left(\rho\left(a_{1}\right) b_{2} \rho\left(a_{2}\right) b_{3}\right)\right. \\
& -\nu\left(b_{1} \rho\left(a_{1}\right) b_{2} \rho\left(a_{2}\right)\right) \nu\left(b_{3}\right)-\nu\left(b_{1} \rho\left(a_{1}\right) b_{2}\right) \nu\left(\rho\left(a_{2}\right) b_{3}\right) \\
& \left.+\nu\left(b_{1}\right) \nu\left(\rho\left(a_{1}\right)\right) \nu\left(b_{2} \rho\left(a_{2}\right) b_{3}\right)+\nu\left(b_{1} \rho\left(a_{1}\right) b_{1}\right) \nu\left(\rho\left(a_{2}\right)\right) \nu\left(b_{3}\right)\right] a_{0} a_{3}
\end{aligned}
$$




$$
\begin{aligned}
& \operatorname{LM}^{\prime}\left(\vec{y}_{3}\right)=\nu\left(b_{1}\right) \nu\left(b_{2}\right) \nu\left(b_{3}\right) a_{0} a_{1} a_{2} a_{3}+\nu\left(b_{1}\right) \nu\left(\stackrel{\circ}{b}_{2} \rho\left(a_{2}\right) \stackrel{\circ}{b}_{3}\right) a_{0} a_{1} a_{3} \\
& +\nu\left(b_{3}\right) \nu\left(\stackrel{\circ}{b}_{1} \rho\left(a_{1}\right) \stackrel{\circ}{b}_{2}\right) a_{0} a_{2} a_{3}+\left[\nu\left(\stackrel{\circ}{b}_{1} \rho\left(a_{1}\right) \stackrel{\circ}{b}_{2} \rho\left(a_{2}\right) \stackrel{\circ}{b}_{3}\right)\right. \\
& -\nu\left(\stackrel{\circ}{b}_{1} \rho\left(a_{1}\right)\right) \nu\left(\stackrel{\circ}{b}_{2} \rho\left(a_{2}\right) \stackrel{\circ}{b}_{3}\right)-\nu\left(\stackrel{\circ}{b}_{1} \rho\left(a_{1}\right) \stackrel{\circ}{b}_{2}\right) \nu\left(\rho\left(a_{2}\right) \stackrel{\circ}{b}_{3}\right) \\
& \left.+\nu\left(b_{2}\right) \nu\left(\stackrel{\circ}{b}_{1} \rho\left(a_{1} a_{2}\right) \stackrel{\circ}{b}_{3}\right)\right] a_{0} a_{3} \\
& =\nu\left(b_{1}\right) \nu\left(b_{2}\right) \nu\left(b_{3}\right) a_{0} a_{1} a_{2} a_{3}+\nu\left(b_{1}\right)\left[\nu\left(b_{2} \rho\left(a_{2}\right) b_{3}\right)-\nu\left(b_{2}\right) \nu\left(\rho\left(a_{2}\right) b_{3}\right)\right. \\
& \left.-\nu\left(b_{3}\right) \nu\left(b_{2} \rho\left(a_{2}\right)\right)+\nu\left(b_{2}\right) \nu\left(b_{3}\right) \nu\left(\rho\left(a_{2}\right)\right)\right] a_{0} a_{1} a_{3}+\nu\left(b_{3}\right)\left[\nu\left(b_{1} \rho\left(a_{1}\right) b_{2}\right)\right. \\
& \left.-\nu\left(b_{1}\right) \nu\left(\rho\left(a_{1}\right) b_{2}\right)-\nu\left(b_{2}\right) \nu\left(b_{1} \rho\left(a_{1}\right)\right)+\nu\left(b_{1}\right) \nu\left(\rho\left(a_{1}\right)\right) \nu\left(b_{2}\right)\right] a_{0} a_{2} a_{3} \\
& +\left[\nu\left(b_{2}\right) \nu\left(b_{1} \rho\left(a_{1} a_{2}\right) b_{3}\right)-\nu\left(b_{1}\right) \nu\left(b_{2}\right) \nu\left(\rho\left(a_{1} a_{2}\right) b_{3}\right)-\nu\left(b_{2}\right) \nu\left(b_{3}\right) \nu\left(b_{1} \rho\left(a_{1} a_{2}\right)\right)\right. \\
& +\nu\left(b_{1}\right) \nu\left(b_{2}\right) \nu\left(b_{3}\right) \nu\left(\rho\left(a_{1} a_{2}\right)\right)+\nu\left(b_{1} \rho\left(a_{1}\right) b_{2} \rho\left(a_{2}\right) b_{3}\right)-\nu\left(b_{1}\right) \nu\left(\rho\left(a_{1}\right) b_{2} \rho\left(a_{2}\right) b_{3}\right) \\
& -\nu\left(b_{2}\right) \nu\left(b_{1} \rho\left(a_{1}\right) \rho\left(a_{2}\right) b_{3}\right)-\nu\left(b_{3}\right) \nu\left(b_{1} \rho\left(a_{1}\right) b_{2} \rho\left(a_{2}\right)\right)+\nu\left(b_{1}\right) \nu\left(b_{2}\right) \nu\left(\rho\left(a_{1}\right) \rho\left(a_{2}\right) b_{3}\right) \\
& +\nu\left(b_{1}\right) \nu\left(b_{3}\right) \nu\left(\rho\left(a_{1}\right) b_{2} \rho\left(a_{2}\right)\right)+\nu\left(b_{2}\right) \nu\left(b_{3}\right) \nu\left(b_{1} \rho\left(a_{1}\right) \rho\left(a_{2}\right)\right) \\
& -\nu\left(b_{1} \rho\left(a_{1}\right)\right) \nu\left(b_{2} \rho\left(a_{2}\right) b_{3}\right)+\nu\left(b_{1}\right) \nu\left(\rho\left(a_{1}\right)\right) \nu\left(b_{2} \rho\left(a_{2}\right) b_{3}\right) \\
& +2 \nu\left(b_{2}\right) \nu\left(b_{1} \rho\left(a_{1}\right)\right) \nu\left(\rho\left(a_{2}\right) b_{3}\right)+\nu\left(b_{3}\right) \nu\left(b_{1} \rho\left(a_{1}\right)\right) \nu\left(b_{2} \rho\left(a_{2}\right)\right) \\
& -2 \nu\left(b_{1}\right) \nu\left(b_{2}\right) \nu\left(\rho\left(a_{1}\right)\right) \nu\left(\rho\left(a_{2}\right) b_{3}\right)-\nu\left(b_{1}\right) \nu\left(b_{3}\right) \nu\left(\rho\left(a_{1}\right)\right) \nu\left(b_{2} \rho\left(a_{2}\right)\right) \\
& -\nu\left(b_{1} \rho\left(a_{1}\right) b_{2}\right) \nu\left(\rho\left(a_{2}\right) b_{3}\right)+\nu\left(b_{1}\right) \nu\left(\rho\left(a_{1}\right) b_{2}\right) \nu\left(\rho\left(a_{2}\right) b_{3}\right) \\
& \left.+\nu\left(b_{3}\right) \nu\left(b_{1} \rho\left(a_{1}\right) b_{2}\right) \nu\left(\rho\left(a_{2}\right)\right)-\nu\left(b_{1}\right) \nu\left(b_{3}\right) \nu\left(\rho\left(a_{1}\right) b_{2}\right) \nu\left(\rho\left(a_{2}\right)\right)\right] a_{0} a_{3}
\end{aligned}
$$




\section{A.5 Moment Polynomials}

For the sake of brevity, we use $1,2,3$ to denote $t_{1}, t_{2}, t_{3}$, with the standing assumption that $0<t_{1}<t_{2}<t_{3}$, and omit listing $a_{1}, \ldots, a_{n}$; hence $\mathfrak{S}(1,0,3,2)$ is an abbreviation for $\mathfrak{S}\left(t_{1}, 0, t_{3}, t_{2} ; a_{1}, a_{2}, a_{3}, a_{4}\right)$, and $\phi_{2-1}$ for $\phi_{t_{2}-t_{1}}$.

After the first few, we omit polynomials in which 0 appears as the first or last index, since the bimodule property easily reduces these to others, viz.

$$
\begin{aligned}
\mathfrak{S}\left(0, s_{1}, \ldots, s_{k} ; a_{0}, a_{1}, \ldots, a_{k}\right) & =a_{0} \phi_{\tau}\left(\mathfrak{S}\left(s_{1}-\tau, \ldots, s_{k}-\tau ; a_{1}, \ldots, a_{k}\right)\right) \\
\mathfrak{S}\left(s_{1}, \ldots, s_{k}, 0 ; a_{1}, \ldots, a_{k}, a_{k+1}\right) & =\phi_{\tau}\left(\mathfrak{S}\left(s_{1}-\tau, \ldots, s_{k}-\tau ; a_{1}, \ldots, a_{k}\right)\right) a_{k+1}
\end{aligned}
$$

where $\tau=\min \left(s_{1}, \ldots, s_{k}\right)$.

We also omit polynomials with consecutive time indices equal, since these can be reduced by multiplying consecutive terms with the same time index; for instance, $\mathfrak{S}\left(t_{1}, t_{1}, t_{2}, t_{3}, t_{3} ; a_{1}, a_{2}, a_{3}, a_{4}, a_{5}\right)=\mathfrak{S}\left(t_{1}, t_{2}, t_{3} ; a_{1} a_{2}, a_{3}, a_{4} a_{5}\right)$. 


$$
\begin{aligned}
& \mathfrak{S}(0)=a_{1} \\
& \mathfrak{S}(0,1)=a_{1} \phi_{1}\left(a_{2}\right) \\
& \mathfrak{S}(1,0)=\phi_{1}\left(a_{1}\right) a_{2} \\
& \mathfrak{S}(0,1,0)=a_{1} \phi_{1}\left(a_{2}\right) a_{3} \\
& \mathfrak{S}(1,0,1)=\phi_{1}\left(a_{1}\right) a_{2} \phi_{1}\left(a_{3}\right)+\omega\left(a_{2}\right)\left[\phi_{1}\left(a_{1} a_{3}\right)-\phi_{1}\left(a_{1}\right) \phi_{1}\left(a_{3}\right)\right] \\
& \mathfrak{S}(0,1,2)=a_{1} \phi_{1}\left(a_{2} \phi_{2-1}\left(a_{3}\right)\right) \\
& \mathfrak{S}(0,2,1)=a_{1} \phi_{1}\left(\phi_{2-1}\left(a_{2}\right) a_{3}\right) \\
& \mathfrak{S}(1,0,2)=\phi_{1}\left(a_{1}\right) a_{2} \phi_{2}\left(a_{3}\right)+\omega\left(a_{2}\right)\left[\phi_{1}\left(a_{1} \phi_{2-1}\left(a_{3}\right)\right)-\phi_{1}\left(a_{1}\right) \phi_{2}\left(a_{3}\right)\right] \\
& \mathfrak{S}(1,2,0)=\phi_{1}\left(a_{1} \phi_{2-1}\left(a_{2}\right)\right) a_{3} \\
& \mathfrak{S}(2,0,1)=\phi_{2}\left(a_{1}\right) a_{2} \phi_{1}\left(a_{3}\right)+\omega\left(a_{2}\right)\left[\phi_{1}\left(\phi_{2-1}\left(a_{1}\right) a_{3}\right)-\phi_{2}\left(a_{1}\right) \phi_{1}\left(a_{3}\right)\right] \\
& \mathfrak{S}(2,1,0)=\phi_{1}\left(\phi_{2-1}\left(a_{1}\right) a_{2}\right) a_{3} \\
& \mathfrak{S}(1,0,1,2)=\phi_{1}\left(a_{1}\right) a_{2} \phi_{1}\left(a_{3} \phi_{3-1}\left(a_{4}\right)\right)+\omega\left(a_{2}\right)\left[\phi_{1}\left(a_{1} a_{3} \phi_{2-1}\left(a_{4}\right)\right)-\phi_{1}\left(a_{1}\right) \phi_{1}\left(a_{3} \phi_{2-1}\left(a_{4}\right)\right)\right] \\
& \mathfrak{S}(1,0,2,1)=\phi_{1}\left(a_{1}\right) a_{2} \phi_{1}\left(\phi_{2-1}\left(a_{3}\right) a_{4}\right)+\omega\left(a_{2}\right)\left[\phi_{1}\left(a_{1} \phi_{2-1}\left(a_{3}\right) a_{4}\right)-\phi_{1}\left(a_{1}\right) \phi_{1}\left(\phi_{2-1}\left(a_{3}\right) a_{4}\right)\right] \\
& \mathfrak{S}(1,2,0,1)=\phi_{1}\left(a_{1} \phi_{2-1}\left(a_{2}\right)\right) a_{3} \phi_{1}\left(a_{4}\right)+\omega\left(a_{3}\right)\left[\phi_{1}\left(a_{1} \phi_{2-1}\left(a_{2}\right) a_{4}\right)-\phi_{1}\left(a_{1} \phi_{2-1}\left(a_{2}\right)\right) \phi_{1}\left(a_{4}\right)\right] \\
& \mathfrak{S}(2,1,0,1)=\phi_{1}\left(\phi_{2-1}\left(a_{1}\right) a_{2}\right) a_{3} \phi_{1}\left(a_{4}\right)+\omega\left(a_{3}\right)\left[\phi_{1}\left(\phi_{2-1}\left(a_{1}\right) a_{2} a_{4}\right)-\phi_{1}\left(\phi_{2-1}\left(a_{1}\right) a_{2}\right) \phi_{1}\left(a_{4}\right)\right] \\
& \mathfrak{S}(1,2,0,2)=\phi_{1}\left(a_{1} \phi_{2-1}\left(a_{2}\right)\right) a_{3} \phi_{2}\left(a_{4}\right)+\omega\left(a_{3}\right)\left[\phi_{1}\left(a_{1} \phi_{2-1}\left(a_{2} a_{4}\right)\right)-\phi_{1}\left(a_{1} \phi_{2-1}\left(a_{3}\right)\right) \phi_{2}\left(a_{4}\right)\right] \\
& \mathfrak{S}(2,0,1,2)=\phi_{2}\left(a_{1}\right) a_{2} \phi_{1}\left(a_{3} \phi_{2-1}\left(a_{4}\right)\right)+\omega\left(a_{2}\right)\left[\phi_{1}\left(\phi_{2-1}\left(a_{1}\right) a_{3} \phi_{2-1}\left(a_{4}\right)\right)-\phi_{2}\left(a_{1}\right) \phi_{1}\left(a_{3} \phi_{2-1}\left(a_{4}\right)\right)\right] \\
& +\omega\left(a_{2}\right) \omega\left(a_{3}\right)\left[\phi_{2}\left(a_{1} a_{4}\right)-\phi_{2}\left(a_{1}\right) \phi_{2}\left(a_{4}\right)\right] \\
& \mathfrak{S}(2,0,2,1)=\phi_{2}\left(a_{1}\right) a_{2} \phi_{1}\left(\phi_{2-1}\left(a_{3}\right) a_{4}\right)+\omega\left(a_{2}\right)\left[\phi_{1}\left(\phi_{2-1}\left(a_{1} a_{3}\right) a_{4}\right)-\phi_{2}\left(a_{1}\right) \phi_{1}\left(\phi_{2-1}\left(a_{3}\right) a_{4}\right)\right] \\
& \mathfrak{S}(2,1,0,2)=\phi_{1}\left(\phi_{2-1}\left(a_{1}\right) a_{2}\right) a_{3} \phi_{2}\left(a_{4}\right)+\omega\left(a_{3}\right)\left[\phi_{1}\left(\phi_{2-1}\left(a_{1}\right) a_{2} \phi_{2-1}\left(a_{4}\right)\right)-\phi_{1}\left(\phi_{2-1}\left(a_{1}\right) a_{2}\right) \phi_{2}\left(a_{4}\right)\right] \\
& +\omega\left(a_{2}\right) \omega\left(a_{3}\right)\left[\phi_{2}\left(a_{1} a_{4}\right)-\phi_{2}\left(a_{1}\right) \phi_{2}\left(a_{4}\right)\right]
\end{aligned}
$$




$$
\begin{aligned}
& \mathfrak{S}(1,0,2,3)=\phi_{1}\left(a_{1}\right) a_{2} \phi_{2}\left(a_{3} \phi_{3-2}\left(a_{4}\right)\right)+\omega\left(a_{2}\right)\left[\phi_{1}\left(a_{1} \phi_{2-1}\left(a_{3} \phi_{3-2}\left(a_{4}\right)\right)\right)-\phi_{1}\left(a_{1}\right) \phi_{2}\left(a_{3} \phi_{3-2}\left(a_{4}\right)\right)\right] \\
& \mathfrak{S}(1,0,3,2)=\phi_{1}\left(a_{1}\right) a_{2} \phi_{2}\left(\phi_{3-2}\left(a_{3}\right) a_{4}\right)+\omega\left(a_{2}\right)\left[\phi_{1}\left(a_{1} \phi_{2-1}\left(\phi_{3-2}\left(a_{3}\right) a_{4}\right)\right)-\phi_{1}\left(a_{1}\right) \phi_{2}\left(\phi_{3-2}\left(a_{3}\right) a_{4}\right)\right] \\
& \mathfrak{S}(1,2,0,3)=\phi_{1}\left(a_{1} \phi_{2-1}\left(a_{2}\right)\right) a_{3} \phi_{3}\left(a_{4}\right)+\omega\left(a_{3}\right)\left[\phi_{1}\left(a_{1} \phi_{2-1}\left(a_{2} \phi_{3-2}\left(a_{4}\right)\right)\right)-\phi_{1}\left(a_{1} \phi_{2-1}\left(a_{2}\right)\right) \phi_{3}\left(a_{4}\right)\right] \\
& \mathfrak{S}(1,3,0,2)=\phi_{1}\left(a_{1} \phi_{2-1}\left(a_{3}\right)\right) a_{3} \phi_{2}\left(a_{4}\right)+\omega\left(a_{3}\right)\left[\phi_{1}\left(a_{1} \phi_{2-1}\left(\phi_{3-2}\left(a_{2}\right) a_{4}\right)\right)-\phi_{1}\left(a_{1} \phi_{3-1}\left(a_{2}\right)\right) \phi_{2}\left(a_{4}\right)\right] \\
& \mathfrak{S}(2,0,1,3)=\phi_{2}\left(a_{1}\right) a_{2} \phi_{1}\left(a_{3} \phi_{3-1}\left(a_{4}\right)\right)+\omega\left(a_{2}\right)\left[\phi_{1}\left(\phi_{2-1}\left(a_{1}\right) a_{3} \phi_{3-1}\left(a_{4}\right)\right)-\phi_{2}\left(a_{1}\right) \phi_{1}\left(a_{3} \phi_{3-1}\left(a_{4}\right)\right)\right] \\
& +\omega\left(a_{2}\right) \omega\left(a_{3}\right)\left[\phi_{2}\left(a_{1} \phi_{3-2}\left(a_{4}\right)\right)-\phi_{2}\left(a_{1}\right) \phi_{3}\left(a_{4}\right)\right] \\
& \mathfrak{S}(2,0,3,1)=\phi_{2}\left(a_{1}\right) a_{2} \phi_{1}\left(\phi_{3-1}\left(a_{3}\right) a_{4}\right)+\omega\left(a_{2}\right)\left[\phi_{1}\left(\phi_{2-1}\left(a_{1} \phi_{3-2}\left(a_{3}\right)\right) a_{4}\right)-\phi_{2}\left(a_{1}\right) \phi_{1}\left(\phi_{3-1}\left(a_{3}\right) a_{4}\right)\right] \\
& \mathfrak{S}(2,1,0,3)=\phi_{1}\left(\phi_{2-1}\left(a_{1}\right) a_{2}\right) a_{3} \phi_{3}\left(a_{4}\right)+\omega\left(a_{3}\right)\left[\phi_{1}\left(\phi_{2-1}\left(a_{1}\right) a_{2} \phi_{3-1}\left(a_{2}\right)\right)-\phi_{1}\left(\phi_{2-1}\left(a_{1}\right) a_{2}\right) \phi_{3}\left(a_{4}\right)\right] \\
& +\omega\left(a_{2}\right) \omega\left(a_{3}\right)\left[\phi_{2}\left(a_{1} \phi_{3-2}\left(a_{4}\right)\right)-\phi_{2}\left(a_{1}\right) \phi_{3}\left(a_{4}\right)\right] \\
& \mathfrak{S}(2,3,0,1)=\phi_{2}\left(a_{1} \phi_{3-2}\left(a_{2}\right)\right) a_{3} \phi_{1}\left(a_{4}\right)+\omega\left(a_{3}\right)\left[\phi_{1}\left(\phi_{2-1}\left(a_{1} \phi_{3-2}\left(a_{3}\right)\right) a_{4}\right)-\phi_{2}\left(a_{1} \phi_{3-2}\left(a_{2}\right)\right) \phi_{1}\left(a_{4}\right)\right] \\
& \mathfrak{S}(3,0,1,2)=\phi_{3}\left(a_{1}\right) \phi_{1}\left(a-3 \phi_{2-1}\left(a_{4}\right)\right)+\omega\left(a_{2}\right)\left[\phi_{1}\left(\phi_{3-1}\left(a_{1}\right) a_{3} \phi_{2-1}\left(a_{4}\right)\right)-\phi_{3}\left(a_{1}\right) \phi_{1}\left(a_{3} \phi_{2-1}\left(a_{4}\right)\right)\right] \\
& +\omega\left(a_{2}\right) \omega\left(a_{3}\right)\left[\phi_{2}\left(\phi_{3-2}\left(a_{1}\right) a_{4}\right)-\phi_{3}\left(a_{1}\right) \phi_{2}\left(a_{4}\right)\right] \\
& \mathfrak{S}(3,0,2,1)=\phi_{3}\left(a_{1}\right) a_{2} \phi_{1}\left(\phi_{2-1}\left(a_{3}\right) a_{4}\right)+\omega\left(a_{2}\right)\left[\phi_{1}\left(\phi_{2-1}\left(\phi_{3-2}\left(a_{1}\right) a_{3}\right) a_{4}\right)-\phi_{3}\left(a_{1}\right) \phi_{1}\left(\phi_{2-1}\left(a_{3}\right) a_{4}\right)\right] \\
& \mathfrak{S}(3,1,0,2)=\phi_{1}\left(\phi_{3-1}\left(a_{1}\right) a_{2}\right) a_{3} \phi_{2}\left(a_{4}\right)+\omega\left(a_{3}\right)\left[\phi_{1}\left(\phi_{3-1}\left(a_{1}\right) a_{2} \phi_{2-1}\left(a_{4}\right)\right)-\phi_{1}\left(\phi_{3-1}\left(a_{1}\right) a_{2}\right) \phi_{2}\left(a_{4}\right)\right] \\
& +\omega\left(a_{2}\right) \omega\left(a_{3}\right)\left[\phi_{2}\left(\phi_{3-2}\left(a_{1}\right) a_{4}\right)-\phi_{3}\left(a_{1}\right) \phi_{2}\left(a_{4}\right)\right]
\end{aligned}
$$$$
\mathfrak{S}(3,2,0,1)=\phi_{2}\left(\phi_{3-2}\left(a_{1}\right) a_{2}\right) a_{3} \phi_{1}\left(a_{4}\right)+\omega\left(a_{3}\right)\left[\phi_{1}\left(\phi_{2-1}\left(\phi_{3-2}\left(a_{1}\right) a_{2}\right) a_{4}\right)-\phi_{2}\left(\phi_{3-2}\left(a_{1}\right) a_{2}\right) \phi_{1}\left(a_{4}\right)\right]
$$ 
To illustrate possibilities of discontinuity, we consider the following for $0<\tau<t_{1}<t_{2}<t_{3}$ :

$$
\begin{aligned}
\mathfrak{S}\left(t_{1}, \tau, t_{3}, 0, t_{2}\right)=\phi_{\tau} & \left(\phi_{t_{1}-\tau}\left(a_{1}\right) a_{2} \phi_{t_{3}-\tau}\left(a_{3}\right)\right) a_{4} \phi_{t_{2}}\left(a_{5}\right) \\
& +\omega\left(a_{2}\right) \phi_{\tau}\left(\phi_{t_{1}-\tau}\left(a_{1} \phi_{t_{3}-t_{1}}\left(a_{3}\right)\right)-\phi_{t_{1}-\tau}\left(a_{1}\right) \phi_{t_{3}-\tau}\left(a_{3}\right)\right) a_{4} \phi_{t_{2}}\left(a_{5}\right) \\
& +\omega\left(a_{4}\right) \phi_{\tau}\left(\phi_{t_{1}-\tau}\left(a_{1}\right) a_{2} \phi_{t_{2}-\tau}\left(\phi_{t_{3}-t_{2}}\left(a_{3}\right) a_{5}\right)\right) \\
& +\omega\left(a_{2}\right) \omega\left(a_{4}\right) \phi_{\tau}\left[\phi_{t_{1}-\tau}\left(a_{1} \phi_{t_{2}-\tau}\left(\phi_{t_{2}-t_{2}}\left(a_{3}\right) a_{5}\right)\right)-\phi_{t_{1}-\tau}\left(a_{1}\right) \phi_{t_{2}-\tau}\left(\phi_{t_{3}-t_{2}}\left(a_{3}\right) a_{5}\right)\right] \\
& -\omega\left(a_{4}\right) \phi_{\tau}\left(\phi_{t_{1}-\tau}\left(a_{1}\right) a_{2} \phi_{t_{3}-\tau}\left(a_{3}\right)\right) \phi_{t_{2}}\left(a_{5}\right) \\
& -\omega\left(a_{2}\right) \omega\left(a_{4}\right) \phi_{\tau}\left(\phi_{t_{1}-\tau}\left(a_{1} \phi_{t_{3}-t_{1}}\left(a_{3}\right)\right)-\phi_{t_{1}-\tau}\left(a_{1}\right) \phi_{t_{3}-\tau}\left(a_{3}\right)\right) \phi_{t_{2}}\left(a_{5}\right)
\end{aligned}
$$

$\mathfrak{S}\left(t_{1}, 0, t_{3}, 0, t_{2}\right)=\phi_{t_{1}}\left(a_{1}\right) a_{2} \phi_{t_{3}}\left(a_{3}\right) a_{4} \phi_{t_{2}}\left(a_{5}\right)$

$$
+\omega\left(a_{2}\right) \omega\left(a_{4}\right)\left[\phi_{t_{1}}\left(a_{1} \phi_{t_{2}-t_{1}}\left(\phi_{t_{3}-t_{2}}\left(a_{3}\right)\right) a_{5}\right)-\phi_{t_{1}}\left(a_{1}\right) \phi_{t_{2}}\left(\phi_{t_{3}-t_{2}}\left(a_{3}\right) a_{5}\right)\right.
$$$$
\left.-\phi_{t_{1}}\left(a_{1} \phi_{t_{3}-t_{1}}\left(a_{3}\right)\right) \phi_{t_{2}}\left(a_{5}\right)+\phi_{t_{1}}\left(a_{1}\right) \phi_{t_{3}}\left(a_{3}\right) \phi_{t_{2}}\left(a_{5}\right)\right]
$$$$
+\omega\left(a_{2}\right)\left[\phi_{t_{1}}\left(a_{1} \phi_{t_{3}-t_{1}}\left(a_{3}\right)\right)-\phi_{t_{1}}\left(a_{1}\right) \phi_{t_{3}}\left(a_{3}\right)\right] a_{4} \phi_{t_{2}}\left(a_{5}\right)
$$$$
+\omega\left(a_{4}\right) \phi_{t_{1}}\left(a_{1}\right) a_{2}\left[\phi_{t_{2}}\left(\phi_{t_{3}-t_{2}}\left(a_{3}\right) a_{5}\right)-\phi_{t_{3}}\left(a_{3}\right) \phi_{t_{2}}\left(a_{5}\right)\right]
$$$$
+\left[\omega\left(a_{2}\right) \omega\left(a_{4}\right) \omega\left(a_{3}\right)-\omega\left(a_{2}\right) \omega\left(\phi_{t_{3}}\left(a_{3}\right) a_{4}\right)-\omega\left(a_{2} \phi_{t_{3}}\left(a_{3}\right)\right) \omega\left(a_{4}\right)\right.
$$$$
\left.+\omega\left(a_{2} \phi_{t_{3}}\left(a_{3}\right) a_{4}\right)\right]\left[\phi_{t_{1}}\left(a_{1} \phi_{t_{2}-t_{1}}\left(a_{5}\right)\right)-\phi_{t_{1}}\left(a_{1}\right) \phi_{t_{3}}\left(a_{3}\right)\right]
$$

$$
\begin{aligned}
\mathfrak{S}\left(t_{1}, 0, t_{3}, 0, t_{2}\right)- & \lim _{\tau \rightarrow 0^{+}} \mathfrak{S}\left(t_{1}, \tau, t_{3}, 0, t_{2}\right)=\left[\omega\left(a_{2}\right) \omega\left(a_{4}\right) \omega\left(a_{3}\right)-\omega\left(a_{2}\right) \omega\left(\phi_{t_{3}}\left(a_{3}\right) a_{4}\right)\right. \\
& \left.\quad-\omega\left(a_{2} \phi_{t_{3}}\left(a_{3}\right)\right) \omega\left(a_{4}\right)+\omega\left(a_{2} \phi_{t_{3}}\left(a_{3}\right) a_{4}\right)\right]\left[\phi_{t_{1}}\left(a_{1} \phi_{t_{2}-t_{1}}\left(a_{5}\right)\right)-\phi_{t_{1}}\left(a_{1}\right) \phi_{t_{3}}\left(a_{3}\right)\right]
\end{aligned}
$$




\section{REFERENCES}

[AFL82] Luigi Accardi, Alberto Frigerio, and John T. Lewis, Quantum stochastic processes, Publ. Res. Inst. Math. Sci. 18 (1982), no. 1, 97-133. MR 660823 (84m:82031)

[AJP06] S. Attal, A. Joye, and C.-A. Pillet (eds.), Open quantum systems. II, Lecture Notes in Mathematics, vol. 1881, Springer-Verlag, Berlin, 2006, The Markovian approach, Lecture notes from the Summer School held in Grenoble, June 16-July 4, 2003. MR 2261250 (2007d:00014)

[Arv89a] William Arveson, Continuous analogues of Fock space, Mem. Amer. Math. Soc. 80 (1989), no. 409, iv+66. MR 987590 (90f:47061)

[Arv89b] Continuous analogues of Fock space. III. Singular states, J. Operator Theory 22 (1989), no. 1, 165-205. MR 1026080 (90m:46118)

[Arv90a] - Continuous analogues of Fock space. II. The spectral $C^{*}$-algebra, J. Funct. Anal. 90 (1990), no. 1, 138-205. MR 1047579 (91d:46073)

[Arv90b] - Continuous analogues of Fock space. IV. Essential states, Acta Math. 164 (1990), no. 3-4, 265-300. MR 1049159 (91d:46074)

[Arv03] _ Noncommutative dynamics and E-semigroups, Springer Monographs in Mathematics, Springer-Verlag, New York, 2003. MR 1978577 (2004g:46082)

[Bha96] B. V. Rajarama Bhat, An index theory for quantum dynamical semigroups, Trans. Amer. Math. Soc. 348 (1996), no. 2, 561-583. MR 1329528 (96g:46059)

[Bil68] Patrick Billingsley, Convergence of probability measures, John Wiley \& Sons Inc., New York, 1968. MR 0233396 (38 \#1718)

[Bla06] B. Blackadar, Operator algebras, Encyclopaedia of Mathematical Sciences, vol. 122, Springer-Verlag, Berlin, 2006, Theory of $C^{*}$-algebras and von Neumann algebras, Operator Algebras and Non-commutative Geometry, III. MR 2188261 (2006k:46082)

[BR87] Ola Bratteli and Derek W. Robinson, Operator algebras and quantum statistical mechanics. 1, second ed., Texts and Monographs in Physics, Springer- 
Verlag, New York, 1987, $C^{*}$ - and $W^{*}$-algebras, symmetry groups, decomposition of states. MR 887100 (88d:46105)

[BS00] B. V. Rajarama Bhat and Michael Skeide, Tensor product systems of Hilbert modules and dilations of completely positive semigroups, Infin. Dimens. Anal. Quantum Probab. Relat. Top. 3 (2000), no. 4, 519-575. MR 1805844 (2001m:46149)

[CE79] Erik Christensen and David E. Evans, Cohomology of operator algebras and quantum dynamical semigroups, J. London Math. Soc. (2) 20 (1979), no. 2, 358-368. MR 551466 (81f:46082)

[CM70] P. Chernoff and J. Marsden, On continuity and smoothness of group actions, Bull. Amer. Math. Soc. 76 (1970), 1044-1049. MR 0265510 (42 \#419)

[Con90] John B. Conway, A course in functional analysis, second ed., Graduate Texts in Mathematics, vol. 96, Springer-Verlag, New York, 1990. MR 1070713 (91e:46001)

[Con00] _ A course in operator theory, Graduate Studies in Mathematics, vol. 21, American Mathematical Society, Providence, RI, 2000. MR 1721402 (2001d:47001)

[Coo47] J. L. B. Cooper, One-parameter semigroups of isometric operators in Hilbert space, Ann. of Math. (2) 48 (1947), 827-842. MR 0027129 (10,257a)

[Dav74] E. B. Davies, Markovian master equations, Comm. Math. Phys. 39 (1974), 91-110. MR 0359633 (50 \#12085)

[Dav76] _ Quantum theory of open systems, Academic Press [Harcourt Brace Jovanovich Publishers], London, 1976. MR 0489429 (58 \#8853)

[DS88] Nelson Dunford and Jacob T. Schwartz, Linear operators. Part I, Wiley Classics Library, John Wiley \& Sons Inc., New York, 1988, General theory, With the assistance of William G. Bade and Robert G. Bartle, Reprint of the 1958 original, A Wiley-Interscience Publication. MR 1009162 (90g:47001a)

[Dug66] James Dugundji, Topology, Allyn and Bacon Inc., Boston, Mass., 1966. MR 0193606 (33 \#1824)

[EL77] D. E. Evans and J. T. Lewis, Dilations of irreversible evolutions in algebraic quantum theory, Comm. Dublin Inst. Adv. Studies Ser. A (1977), no. 24, v+104. MR 0489494 (58 \#8915) 
[Emc78] Gérard G. Emch, Minimal dilations of CP-flows, C*-algebras and applications to physics (Proc. Second Japan-USA Sem., Los Angeles, Calif., 1977), Lecture Notes in Math., vol. 650, Springer, Berlin, 1978, pp. 156-159. MR 504761 (81a:46079)

[EN06] Klaus-Jochen Engel and Rainer Nagel, A short course on operator semigroups, Universitext, Springer, New York, 2006. MR 2229872 (2007e:47001)

[GKS76] Vittorio Gorini, Andrzej Kossakowski, and E. C. G. Sudarshan, Completely positive dynamical semigroups of $N$-level systems, J. Mathematical Phys. 17 (1976), no. 5, 821-825. MR 0406206 (53 \#9998)

[Haa73] Fritz Haake, Statistical treatment of open systems by generalized master equations, Quantum Statistics in Optics and Solid-State Physics, Springer Tracts in Modern Physics, vol. 66, Springer Berlin Heidelberg, 1973, pp. 98168.

[HP57] Einar Hille and Ralph S. Phillips, Functional analysis and semi-groups, American Mathematical Society Colloquium Publications, vol. 31, American Mathematical Society, Providence, R. I., 1957, rev. ed. MR 0089373 $(19,664 d)$

[Kak45] Shizuo Kakutani, Markoff process and the Dirichlet problem, Proc. Japan Acad. 21 (1945), 227-233 (1949). MR 0032866 (11,357h)

[Kal97] Olav Kallenberg, Foundations of modern probability, Probability and its Applications (New York), Springer-Verlag, New York, 1997. MR 1464694 (99e:60001)

[Kas80] G. G. Kasparov, Hilbert $C^{*}$-modules: theorems of Stinespring and Voiculescu, J. Operator Theory 4 (1980), no. 1, 133-150. MR 587371 (82b:46074)

[KR83] Richard V. Kadison and John R. Ringrose, Fundamentals of the theory of operator algebras. Vol. I, Pure and Applied Mathematics, vol. 100, Academic Press Inc. [Harcourt Brace Jovanovich Publishers], New York, 1983, Elementary theory. MR 719020 (85j:46099)

[KR86] - Fundamentals of the theory of operator algebras. Vol. II, Pure and Applied Mathematics, vol. 100, Academic Press Inc., Orlando, FL, 1986, Advanced theory. MR 859186 (88d:46106)

[Küm85] Burkhard Kümmerer, Markov dilations on $W^{*}$-algebras, J. Funct. Anal. 63 (1985), no. 2, 139-177. MR 803091 (87b:46070) 
[Lan95] E. C. Lance, Hilbert $C^{*}$-modules, London Mathematical Society Lecture Note Series, vol. 210, Cambridge University Press, Cambridge, 1995, A toolkit for operator algebraists. MR 1325694 (96k:46100)

[Law74] J. D. Lawson, Joint continuity in semitopological semigroups, Illinois J. Math. 18 (1974), 275-285. MR 0335674 (49 \#454)

[Lin76] G. Lindblad, On the generators of quantum dynamical semigroups, Comm. Math. Phys. 48 (1976), no. 2, 119-130. MR 0413878 (54 \#1990)

[Mac09] Barbara D. MacCluer, Elementary functional analysis, Graduate Texts in Mathematics, vol. 253, Springer, New York, 2009. MR 2462971 (2010b:46001)

[MS02] Paul S. Muhly and Baruch Solel, Quantum Markov processes (correspondences and dilations), Internat. J. Math. 13 (2002), no. 8, 863-906. MR 1928802 (2003j:46100)

[MS10] Daniel Markiewicz and Orr Moshe Shalit, Continuity of CP-semigroups in the point-strong operator topology, J. Operator Theory 64 (2010), no. 1, 149-154. MR 2669432 (2011j:46115)

[MT05] V. M. Manuilov and E. V. Troitsky, Hilbert $C^{*}$-modules, Translations of Mathematical Monographs, vol. 226, American Mathematical Society, Providence, RI, 2005, Translated from the 2001 Russian original by the authors. MR 2125398 (2005m:46099)

[NS06] Alexandru Nica and Roland Speicher, Lectures on the combinatorics of free probability, London Mathematical Society Lecture Note Series, vol. 335, Cambridge University Press, Cambridge, 2006. MR 2266879 (2008k:46198)

[Pas73] William L. Paschke, Inner product modules over $B^{*}$-algebras, Trans. Amer. Math. Soc. 182 (1973), 443-468. MR 0355613 (50 \#8087)

[Pau02] Vern Paulsen, Completely bounded maps and operator algebras, Cambridge Studies in Advanced Mathematics, vol. 78, Cambridge University Press, Cambridge, 2002. MR 1976867 (2004c:46118)

[Pow88] Robert T. Powers, An index theory for semigroups of *-endomorphisms of $\mathcal{B}(\mathcal{H})$ and type $I I_{1}$ factors, Canad. J. Math. 40 (1988), no. 1, 86-114. MR 928215 (89f:46116)

[Rie74] Marc A. Rieffel, Induced representations of $C^{*}$-algebras, Advances in Math. 13 (1974), 176-257. MR 0353003 (50 \#5489) 
[Roy88] H. L. Royden, Real analysis, third ed., Macmillan Publishing Company, New York, 1988. MR 1013117 (90g:00004)

[RW98] Iain Raeburn and Dana P. Williams, Morita equivalence and continuoustrace $C^{*}$-algebras, Mathematical Surveys and Monographs, vol. 60, American Mathematical Society, Providence, RI, 1998. MR 1634408 (2000c:46108)

[Sak98] Shôichirô Sakai, $C^{*}$-algebras and $W^{*}$-algebras, Classics in Mathematics, Springer-Verlag, Berlin, 1998, Reprint of the 1971 edition. MR 1490835 (98k:46085)

[Sau86] Jean-Luc Sauvageot, Markov quantum semigroups admit covariant Markov $C^{*}$-dilations, Comm. Math. Phys. 106 (1986), no. 1, 91-103. MR 853979 (88a:46077)

[Sau88] _ First exit time: a theory of stopping times in quantum processes, Quantum probability and applications, III (Oberwolfach, 1987), Lecture Notes in Math., vol. 1303, Springer, Berlin, 1988, pp. 285-299. MR 985827 (90e:46054)

[Sau91] _ Le problème de Dirichlet dans les $C^{*}$-algèbres, J. Funct. Anal. 101 (1991), no. 1, 50-73. MR 1132307 (92j:46113)

[Sel97] Dylan Bernard Selegue, Minimal dilations of CP maps and a $C^{*}$-extension of the Szego limit theorem, ProQuest LLC, Ann Arbor, MI, 1997, Thesis (Ph.D.)-University of California, Berkeley. MR 2696312

[Sha08] Orr Moshe Shalit, $\mathrm{E}_{0}$-dilation of strongly commuting $\mathrm{CP}_{0}$-semigroups, J. Funct. Anal. 255 (2008), no. 1, 46-89. MR 2417809 (2009b:46123)

[Ska02] M. Skaŭde, Dilations, product systems, and weak dilations, Mat. Zametki 71 (2002), no. 6, 914-923. MR 1933111 (2003h:46103)

[Ske06] Michael Skeide, The index of (white) noises and their product systems, Infin. Dimens. Anal. Quantum Probab. Relat. Top. 9 (2006), no. 4, 617-655. MR 2282723 (2007m:46109)

[Sti55] W. Forrest Stinespring, Positive functions on $C^{*}$-algebras, Proc. Amer. Math. Soc. 6 (1955), 211-216. MR 0069403 (16,1033b)

[Tom57] Jun Tomiyama, On the projection of norm one in $W^{*}$-algebras, Proc. Japan Acad. 33 (1957), 608-612. MR 0096140 (20 \#2635) 
[VDN92] D. V. Voiculescu, K. J. Dykema, and A. Nica, Free random variables, CRM Monograph Series, vol. 1, American Mathematical Society, Providence, RI, 1992, A noncommutative probability approach to free products with applications to random matrices, operator algebras and harmonic analysis on free groups. MR 1217253 (94c:46133)

[Voi85] Dan Voiculescu, Symmetries of some reduced free product $C^{*}$-algebras, Operator algebras and their connections with topology and ergodic theory (Buşteni, 1983), Lecture Notes in Math., vol. 1132, Springer, Berlin, 1985, pp. 556-588. MR 799593 (87d:46075)

[Voi99] - The analogues of entropy and of Fisher's information measure in free probability theory. VI. Liberation and mutual free information, Adv. Math. 146 (1999), no. 2, 101-166. MR 1711843 (2001a:46064)

[VS84] G. F. Vincent-Smith, Dilation of a dissipative quantum dynamical system to a quantum Markov process, Proc. London Math. Soc. (3) 49 (1984), no. 1, 58-72. MR 743370 (85i:46086)

[Zhu93] Ke He Zhu, An introduction to operator algebras, Studies in Advanced Mathematics, CRC Press, Boca Raton, FL, 1993. MR 1257406 (94k:46124) 